Andreas Frewer, Heiner Bielefeldt ( $\mathrm{Hg}$.)

\title{
Das Menschenrecht auf Gesundheit
}

Normative Grundlagen und aktuelle Diskurse 
Andreas Frewer, Heiner Bielefeldt (Hg.)

Das Menschenrecht auf Gesundheit

Menschenrechte in der Medizin | Human Rights in Healthcare | Band I hrsg. von Prof. Dr. Dr. Heiner Bielefeldt und Prof. Dr. Andreas Frewer, M.A. 

Andreas Frewer, Heiner Bielefeldt (Hg.)

\section{Das Menschenrecht auf Gesundheit}

Normative Grundlagen und aktuelle Diskurse

[transcript] 
Ergebnisse des Forschungsprojekts »Human Rights in Healthcare« Mit freundlicher Unterstützung der Emerging Fields Initiative (EFI) und der Friedrich-Alexander-Universität Erlangen-Nürnberg (FAU)

\section{(c) $(1) \Theta(\Theta$}

Dieses Werk ist lizenziert unter der Creative Commons Attribution-NonCommercial-NoDerivs 4.0 Lizenz (BY-NC-ND). Diese Lizenz erlaubt die private Nutzung, gestattet aber keine Bearbeitung und keine kommerzielle Nutzung. Weitere Informationen finden Sie unter https://creativecommons.org/licenses/by-nc-nd/4.o/deed.de/.

Um Genehmigungen für Adaptionen, Übersetzungen, Derivate oder Wiederverwendung zu kommerziellen Zwecken einzuholen, wenden Sie sich bitte an rights@transcript-verlag.de

\section{(๔ 2016 transcript Verlag, Bielefeld}

\section{Bibliografische Information der Deutschen Nationalbibliothek}

Die Deutsche Nationalbibliothek verzeichnet diese Publikation in der Deutschen Nationalbibliografie; detaillierte bibliografische Daten sind im Internet über http://dnb.d-nb.de abrufbar.

Umschlaggestaltung: Kordula Röckenhaus, Bielefeld Druck: Majuskel Medienproduktion GmbH, Wetzlar Print-ISBN 978-3-8376-3471-6 PDF-ISBN 978-3-8394-3471-0

Gedruckt auf alterungsbeständigem Papier mit chlorfrei gebleichtem Zellstoff. Besuchen Sie uns im Internet: http://www.transcript-verlag.de Bitte fordern Sie unser Gesamtverzeichnis und andere Broschüren an unter: info@transcript-verlag.de 


\section{Inhalt}

Menschen, Rechte und Medizin.

\section{Zur Einführung}

Andreas Frewer, Heiner Bielefeldt |7

Der Menschenrechtsansatz im Gesundheitswesen.

Einige Grundsatzüberlegungen

Heiner Bielefeldt | 19

Das Menschenrecht auf Gesundheit.

Grundzüge eines komplexen Rechts

Michael Krennerich | 57

Das Recht auf Gesundheit in der Praxis.

Von der Forschung zur internationalen Therapie

Andreas Frewer $\mid 93$

Die Konkretisierung von Kernbereichen des

Menschenrechts auf Gesundheit. Internationale

Debatten zu »Minimum Core Obligations»

Amrei Müller | 125

Universelle Gesundheitssicherung.

Konzeptionelle Grundlagen und der

Beitrag Nationaler Ethikräte

Christina Heinicke, Lotta Eriksson,

Abha Saxena, Andreas Reis | 169 
Ambivalenzen der Medikalisierung.

Ein Plädoyer für das Ernstnehmen der subjektiven

Perspektive im Umgang mit Gesundheit und Krankheit

Martina Schmidhuber | 195

Gibt es ein Recht auf Krankheit?

Historische und theoretische Überlegungen

im Anschluss an Juli Zehs Roman Corpus Delicti

Caroline Welsh | 215

\section{ANHANG}

Allgemeine Bemerkung Nr. 14:

Das Recht auf ein Höchstmaß an Gesundheit (Artikel 12).

(General Comment 14)

UN-Ausschuss für wirtschaftliche,

soziale und kulturelle Menschenrechte | 241

Autorinnen und Autoren | 277 


\title{
Menschen, Rechte und Medizin
}

\author{
Zur Einführung
}

ANDREAS FREWER, HEINER BIELEFELDT

\section{Medizin Und MenschenReChte}

Themen im Spannungsfeld von Medizin und Menschenrechten umreißen ein Spektrum höchst aktueller wie auch brisanter Fragen: Auf welche Weise wird das Menschenrecht auf Gesundheit für Patienten, sozial Schwache, Behinderte, Flüchtlinge oder »Menschen ohne Papiere « umgesetzt? ${ }^{1}$ Wer versorgt die Migrantinnen und Migranten in medizinischer Hinsicht? Wie können nicht nur Leib und Leben gesichert, sondern auch die Unterbringung und gesundheitliche Unterstützung adäquat gewährleistet werden? Globalisierung wie auch neue Migrationsbewegungen bringen hier eine Fülle von Detailproblemen, die im Kern viele Aspekte körperlicher und psychischer Versorgung vulnerabler Menschen betreffen, insbesondere nach traumatischen Erfahrungen in den Heimatländern, bei Krieg, Krisen oder Katastrophen. Humanitäre Hilfe bei dramatischen Naturphänomenen und seelische Betreuung zur langfristigen Genesung wie auch Integration traumatisierter Kranker berühren zentrale Ansprüche menschenrechtlicher Praxisarbeit. Man braucht aber nicht nur die internationalen Dimensionen

1 Vgl. u.a. Grover (2011) und Mylius et al. (2011). 
der Menschenrechte in den Blick zu nehmen, ${ }^{2}$ sondern bei vielen Themen der Medizin stellen sich auch innerhalb einzelner Länder sofort menschenrechtliche und moralische Fragen: Wie kann bei der Verteilung von Organen Gerechtigkeit gewährleistet werden? Nicht zuletzt die bekannt gewordenen Transplantationsskandale bei der Allokation von knappen Gütern haben grundlegende menschenrechtliche wie moralische Probleme offenbar werden lassen. Sind die Kriterien für Wartelisten nicht nur medizinisch korrekt gestaltet, sondern auch ethisch reflektiert und für alle Menschen transparent wie auch nachvollziehbar? Können Patientinnen und Patienten in ihrer Autonomie bei der (Lebend-)Spende von Organen bzw. bei ihren Ansprüchen als Anwärter für Therapien ausreichend unterstützt werden? Auch hier sollten die Disziplinen, Medizin, Ethik, Menschenrechte und Politik zweifellos noch viel enger zusammenarbeiten.

Neuregelungen zum Lebensende - vor kurzem etwa mit Gesetzen zu besserer Hospiz- und Palliativversorgung oder dem Verbot kommerzialisierter Suizidhilfe - berühren sofort nicht nur urmenschliche, sondern auch moralische und (menschen)rechtliche Dimensionen gesellschaftlichen $\mathrm{Zu}$ sammenlebens. Wie kann das Recht auf Gesundheit für ältere, hochbetagte oder sterbende Personen gewährleistet werden? Welche Hilfen sind in Pflege, ambulanter wie auch stationärer Versorgung in einem immer stärker durch ökonomische Rahmenbedingungen geprägten Gesundheitswesen relevant? Rechtsansprüche als Mitglieder einer gesetzlichen oder privaten Krankenversicherung, Schutz besonders vulnerabler Patientengruppen und gerechte Vergütungsinstrumente - sofort sind die Fragen von Menschenrechten und Moral im Gespräch, geht es doch auch oft um die Inklusion von Behinderten, menschenwürdige Umstände im Sterben oder beim Umgang mit dem (hirn)toten Körper. Hinzu kommen alle Aspekte sexueller oder reproduktiver Freiheit, die ebenfalls mit dem Verbot von Stigmatisierung und Diskriminierung sofort auf einer menschenrechtlichen Ebene sind.

Wissenschaft und Forschung erreichen dabei zudem immer neue Grenzen, sei es bei Stammzell- und Embryonenforschung, Gene-Editing oder Präimplantationsdiagnostik - wann wird die Menschenwürde verletzt? Welche Humanexperimente der modernen Medizin sind ethisch erlaubt?

$2 \mathrm{Zu}$ internationalen Initiativen im Bereich »Medicine and Human Rights« bzw. "Right to Health" siehe insbesondere Mann et al. (1999), Farmer (2005), Gruskin et al. (2005), London (2008), Toebes et al. (2012) sowie Toebes (2014). 
Wann überschreiten Versuche am Menschen moralische Grenzen, die durch nationale Gesetze oder internationale Deklarationen geregelt werden bzw. geregelt werden sollten? ${ }^{3}$

Auch jenseits juristischer Fragen stellen sich gerade für die Humanmedizin immer wieder neue Probleme mit politischem Regelungsbedarf und grundsätzlichen anthropologischen Dimensionen. Im Gesundheitswesen ergeben sich zudem mögliche Gewissenskonflikte für das professionelle Handeln: Wann können Hebammen, Pflegende, Ärztinnen und Ärzte aus religiösen oder Wertüberzeugungen Maßnahmen verweigern, etwa die Beteiligung an Abtreibungen aus Gewissensgründen? In pluralistischen Gesellschaften treffen nicht selten unterschiedliche Konfessionen im klinischen Alltag aufeinander: Eine muslimische Ärztin arbeitet beispielsweise nicht nur eng mit christlichen oder agnostischen Krankenschwestern oder Kollegen anderer Konfessionen zusammen, respektiert Behandlungswünsche jüdischer Patienten oder Werte von Zeugen Jehovas bei Bluttransfusionen und versucht beim gemeinsamen Handeln einen Konsens verschiedenster Perspektiven zu schaffen; dabei können zahlreiche moralisch und menschenrechtlich relevante Fragen oder Probleme auftreten.

\section{Forschung zU MenschenReChten in DER Medizin}

Genau das sind einige der Themen und Arbeitsfelder, die sich eine Forschungsgruppe an der Friedrich-Alexander-Universität Erlangen-Nürnberg zum Schwerpunkt gewählt hat: Im Rahmen der »Emerging Fields Initiative« (EFI) wird das Projekt »Human Rights in Healthcare« als interdisziplinäres Gebiet zwischen Philosophischer Fakultät mit Fachbereich Theologie, Medizinischer und Rechtswissenschaftlicher Fakultät gefördert. ${ }^{4}$ Im Zeitraum von 2014 bis 2017 arbeitet eine Gruppe von Professorinnen und Professoren, Fellows und wissenschaftlichen Mitarbeiterinnen und Mitarbeitern eng zusammen, um Fragen der Menschenrechte für Medizin und Gesundheitswesen zu vertiefen. Das Spektrum der Arbeitsgruppen reicht dabei von menschenrechtlich-philosophischen,

3 Vgl. u.a. Frewer et al. (2009b), Bielefeldt (2011) und Frewer/Schmidt (2014).

4 Siehe www.efi.fau.de/projekte/human-rights-in-healthcare/ [10.12.2015]. 
klinisch-ethischen über rechtswissenschaftliche bis zu politologischsozialwissenschaftlichen Themen.

Der vorliegende Band soll zum Start der neuen Fachbuchreihe einige Grundlagen ${ }^{5}$ für das sich in der Tat sehr stark entwickelnde Feld darstellen; aus der Zusammenarbeit bei Workshops, ${ }^{6}$ nationalen ${ }^{7}$ und internationalen Fachtagungen ${ }^{8}$ hervorgegangen und durch gemeinsame Diskussionen vertieft, sollen die Beiträge einen Überblick geben sowie das Feld in Theorie und Praxis abstecken. Weitere nachfolgende Bände werden Themen wie die Behandlung von Menschen in besonders vulnerablen Lebenssituationen etwa Migranten, Flüchtlinge, Behinderte oder Kranke am Lebensende ausdifferenzieren. ${ }^{9}$

Dies sind die Schwerpunkte des vorliegenden Bandes der neuen Reihe: Heiner Bielefeldt stellt Grundsatzüberlegungen zum Menschenrechtsansatz im Gesundheitswesen vor. Er zeigt Klärungsbedarf auf und erläutert in der Folge ein universalistisches Verständnis von Menschenwürde und Menschenrechten. Bielefeldt verdeutlicht die enge Beziehung von Menschenrechten und Medizin, wobei Studien zum Wissensstand über Menschenrechte in Deutschland - selbst in der Ärzteschaft - deutlich gemacht haben, dass das Recht auf Gesundheit unterrepräsentiert ist; hier seien präzise transdisziplinäre Übersetzungsprozesse zu leisten. Der Artikel erläutert normative Prinzipien und den Universalismus der Menschenrechte (Kap. 2) sowie auch ihre freiheitliche Orientierung, prägnant formuliert im Anspruch auf Respekt und Förderung der Autonomie jedes Menschen, gerade auch in Fällen von Krankheit oder Behinderung (Kap. 3). Des Weiteren

5 Vgl. zu Kontexten insbesondere Bielefeldt (1998) und Frewer et al. (2009b).

6 Kick-Off-Workshop »Human Rights in Healthcare« im Nürnberger Menschenrechtszentrum (26.04.2014) und die große universitäre FAU-Ringvorlesung »Menschenrechte im Gesundheitswesen. Personen in vulnerablen Situationen« an der Universität Erlangen-Nürnberg (Sommersemester 2015).

7 Die Tagung »Autonomie und Menschenrechte am Lebensende« in Erlangen (21.-22.11.2014) und die nationale Konferenz »Sich für die Gesundheit stark machen!« im Bundesministerium für Wirtschaftliche Zusammenarbeit in Berlin (26.-27.03.2015).

8 Internationale EFI Conference »The Right to Health - an Empty Promise?« in der Berlin-Brandenburgischen Akademie der Wissenschaften (14.-16.09.2015).

9 Vgl. Bielefeldt (2009a), Frewer et al. (2009a) und Bielefeldt et al. (2016). 
erörtert Bielefeldt den Gleichheitsgrundsatz, ausdifferenziert in Gestalt spezifischer Diskriminierungsverbote (Kap. 4), und die menschenrechtliche Gewährleistungsfunktion des Staates (Kap. 5). Damit umreißt er Freiheitsund Gleichheitsrechte, für deren Verwirklichung die Staaten im internationalen Recht verantwortlich sind. Der Beitrag betont den engen Zusammenhang zwischen dem Recht auf Gesundheit und anderen Menschenrechten u.a. Bildung, Sicherheit, Privatsphäre und Religionsfreiheit.

Michael Krennerich verankert das Menschenrecht auf Gesundheit zunächst im Völkerrecht und zeigt die Wurzeln bei der Entstehung im 20. Jahrhundert. Daran anknüpfend erläutert der Autor Grundzüge des Menschenrechts auf Gesundheit und fächert relevante Quellen auf, insbesondere ausgehend vom UN-Sozialpakt über wirtschaftliche, soziale und kulturelle Rechte (Kap. 2). Im dritten Abschnitt erörtert Krennerich die Trias staatlicher Pflichten zur Achtung (obligation to respect), zum Schutz (obligation to protect) sowie zur Gewährleistung (obligation to fulfil) der Menschenrechte; überdies geht er ein auf internationale Dimensionen des Rechts auf Gesundheit und seiner Umsetzung (Kap. 4). Abschließend wird das notwendige Empowerment zur effektiven Einforderung menschenrechtlicher Ansprüche bei den jeweiligen Staaten skizziert.

Andreas Frewer setzt das Menschenrecht auf Gesundheit in Bezug zur medizinischen Praxis; dabei werden Fragen der Forschung am Menschen und internationale Strategien zur Verbesserung gesundheitlicher Grundversorgung beleuchtet. Der menschenrechtliche Schutz von Patienten und Probanden wird in seiner historischen Entwicklung rekonstruiert (Kap. 2) sowie in Bezug auf zentrale moralische Grenzfragen erläutert (Kap. 3). Neben dem Menschenrecht auf körperliche Unversehrtheit und der Zustimmung nach Aufklärung (»informed consent«) geht es auch um die Praxis der Umsetzung (»Therapie«) in internationaler Perspektive (Kap. 4) und daraus resultierende Konsequenzen in Schlussüberlegungen (Kap. 5).

Amrei Müller erörtert das Konzept des Kernbereichs (Minimum Core Obligations) für das Recht auf Gesundheit. Zunächst wird die Idee für den Ansatz eines Kernbereichs umrissen (Kap. 2), bevor die Probleme der vorherrschenden Konzeption dieses Ansatzes analysiert werden (Kap. 3). Auf Basis der Erklärungen des UN-Sozialausschusses und anderer Institutionen sowie der (inter)nationalen Rechtsprechung entwickelt die Autorin sodann ein Konzept, das den Kernbereichen des Rechts auf Gesundheit greifbare Konturen auf universaler Ebene gibt und gleichzeitig den unter- 
schiedlichen Voraussetzungen einzelner Staaten pragmatisch Rechnung trägt (Kap. 4). In ihren Schlussüberlegungen werden zentrale Positionen und Konsequenzen der Minimum Core Obligations für das Recht auf Gesundheit zusammengefasst (Kap. 5).

Christina Heinicke, Lotta Eriksson, Abha Saxena und Andreas Reis beleuchten das Thema »Universelle Gesundheitssicherung (UGS/UHC). Nach Darstellung von Grundlagen (Kap. 2) werden ethische Herausforderungen bei der Umsetzung erörtert (Kap. 3). Im Spiegel einer Umfrage zu den Positionen Nationaler Ethikräte zur Einführung des Konzepts Universal Health Coverage (UHC) werden zudem Desiderate globaler medizinischer Standards untersucht (Kap. 4). Speziell illustrieren die Autoren die Probleme universeller Gesundheitssicherung am Beispiel von den Niederlanden wie auch Thailand und werfen abschließend einen Blick auf mögliche Entwicklungen (Kap. 5).

Martina Schmidhuber analysiert Ambivalenzen der starken Entwicklung der Medizin und ihres Einflusses auf Mensch wie auch Gesellschaft (»Medikalisierung«); sie plädiert dabei für ein Ernstnehmen individueller Perspektiven im Umgang mit Gesundheit, Krankheit und Leid. Die Autorin argumentiert, dass Heilkunde und Naturwissenschaft versuchen, objektive Fakten zur Verfügung zu stellen, innerhalb derer jedoch die subjektive Sicht von Betroffenen und Patienten berücksichtigt werden muss. Nach der Darstellung normativer und medizintheoretischer Grundlagen (Kap. 1) betrachtet der Beitrag exemplarisch drei Felder von aktueller und gesellschaftlicher Relevanz (Kap. 2). Bei ihren Überlegungen lässt sich die Autorin vom Verbot der Diskriminierung bzw. Stigmatisierung leiten und hinterfragt anthropologische Dimensionen von Krankheit und Sterblichkeit. Sie zeigt, dass ein Recht auf das erreichbare Höchstmaß an körperlicher und geistiger Gesundheit nicht bedeutet, dass es leidfreies Leben gibt.

Der Beitrag von Caroline Welsh stellt für das Menschenrecht auf Gesundheit provokativ die Gegenfrage: Brauchen wir womöglich ein Recht auf Krankheit? Ausgehend von dem literarischen Werk »Corpus Delicti. Ein Prozess« von Juli Zeh (2009) mit der Fiktion einer zukünftigen Gesundheitsdiktatur im 21. Jahrhundert stellt sie Überlegungen zu ambivalenten Auswirkungen des medizinischen Fortschritts an. Der Aufsatz rekonstruiert zunächst Diskurse in der Entwicklung von »umfassender « zu "vollkommener" Gesundheit (Kap. 2) und bezieht sich dabei auf historische Meilensteine im Verständnis von Gesundheitsrechten, die möglicher- 
weise aber auch Pflichten zur persönlichen Gesundheitspflege normieren könnten (Kap. 3). Anschließend kontextualisiert die Autorin das Recht auf Gesundheit und als kritisches Korrektiv verstandenes Recht auf Krankheit im menschenrechtlichen Kontext (Kap. 4), insbesondere mit Blick auf zentrale Dokumente und die Gesundheitsdefinition der WHO. Im Schlusskapitel 5 schlägt Welsh den Bogen zurück von Gesundheitsregelungen zu gesellschaftlichen Dystopien in der Literatur und unterstreicht die Bedeutung der Freiheitsrechte für menschliches Zusammenleben.

Im Anhang findet sich in voller Länge ein Schlüsseldokument für die Verankerung der Menschenrechte im Gesundheitswesen. Neben der gut bekannten Allgemeinen Erklärung der Menschenrechte (1948) und dem Internationalen Pakt über wirtschaftliche, soziale und kulturelle Rechte (»UN-Sozialpakt«) aus dem Jahr 1966 steht der General Comment Nr. 14 (2000) für wichtige rechtsdogmatische Klarstellungen zum Recht auf Gesundheit. ${ }^{10}$ Da dieser Text in mehreren Aufsätzen des Bandes zitiert wird, aber eine deutsche Fassung im Internet nicht verfügbar ist, haben wir ihn hier nochmals im Anhang in voller Länge wiedergegeben. ${ }^{11}$

Vor genau 20 Jahren hat Amnesty International Deutschland den Band »Diagnose: akutes Herzversagen«. Heilberufler und Menschenrechtsverletzungen herausgegeben. ${ }^{12}$ Dort wird die schwierige »Gratwanderung zwischen Gewissen, Gefährdung und Komplizenschaft« beschrieben, wenn Mediziner in besonderen Situationen eben nicht die Rechte ihrer Patienten im Blick haben, sondern formale staatliche oder ökonomische Zwänge im Sinne einer »dual loyalty«. Auf diese Weise lassen Heilende nicht nur wertschätzende Empathie oder herzliche Anteilnahme vermissen, sondern können zu (Mit)Tätern bei Menschenrechtsverletzungen etwa durch Forschung oder Folter werden. ${ }^{13}$ Die Ärzteschaft wie auch die Gesellschaft des 21. Jahrhunderts braucht in Bezug auf die Menschenrechte in besonderer Weise »Herz und Verstand«. Natürlich ist es nicht nur eine Frage von Emotio und Ratio, sondern eine Aufgabe der internationalen Staaten; für den Einzelnen bleiben jedoch das sensible Wahrnehmen von moralisch

10 Vgl. UN General Assembly (1966) und Committee on Economic, Social, and Cultural Rights (2000).

11 Wir danken dem Nomos-Verlag für die Erlaubnis zum Wiederabdruck.

12 Amnesty International (1996).

13 Vgl. u.a. Frewer et al. (2009a). 
schwierigen Situationen und ein engagiertes Eintreten für die Rechte schwächerer Gruppen sowie auch klare Analysen und kritische Diskussionen zu den menschenrechtlichen Herausforderungen notwendig. ${ }^{14}$ Dabei darf keineswegs die besondere Geschichte des Bereichs Medizin und Menschenrechte - gerade in Deutschland - vergessen werden, die sogar in der Region der Universität Erlangen-Nürnberg zentrale Entwicklungen und Ereignisse gesehen hat: ${ }^{15}$ Die Nürnberger »Rassegesetze« im »Dritten Reich « des Nationalsozialismus und das internationale Tribunal der Nürnberger Prozesse, insbesondere das Nachfolgeverfahren des Ärzteprozesses mit Verabschiedung des »Nuremberg Code of Medical Ethics« (1947). Nürnberg hat als "Stadt des Friedens und der Menschenrechte« die historische Bedeutung und Verantwortung positiv aufgegriffen: Zahlreiche Initiativen wie auch Aktivitäten vom Menschenrechtszentrum über das Menschenrechtsbüro und die »Internationale Akademie Nürnberger Prinzipien « bis hin zur Durchführung von Filmfestivals für Menschenrechte und die Verleihung eines Menschenrechtspreises haben in den letzten Jahren eine dichte Kultur der Auseinandersetzung mit grundlegenden Dimensionen der Menschenrechte geschaffen. Sichtbarster Ausdruck sind die "Straße der Menschenrechte « mit den 30 Artikeln der »Universal Declaration of Human Rights« und die regelmäßig durchgeführte »Friedenstafel«. In Bezug auf die Fragen der Menschenrechte im Gesundheitswesen sind für Erlangen neben dem Lehrstuhl für Menschenrechte und Menschenrechtspolitik die jährlichen "Ethiktage« und die Kongressreihe "Medizin und Gewissen« hervorzuheben, die seit 20 Jahren zwischen Nürnberg und Erlangen alterniert, als kritische Foren zur Reflektion von Medizin, Geschichte, Ethik und Menschenrechten. Im Herbst 2015 ist zudem ein Interdisziplinäres Zentrum für Menschenrechte an der FAU (Centre for Human Rights Erlangen-Nürnberg/»CHREN«) entstanden, das ein breites Spektrum der relevanten Fragen bearbeiten soll. ${ }^{16}$ Mit dem vorliegenden Band und der gesamten Buchreihe soll der fachliche Austausch im Spannungsfeld von Medizin und Menschenrechten nochmals vertieft sowie auf eine breite transdisziplinäre Basis gestellt werden.

14 Vgl. WHO (2002), UN Development Group (2003), Yamin (2008), Krajewski (2010), Rothhaar/Frewer (2012) und Ooms et al. (2014).

15 Vgl. u.a. Council of Europe (1950), Frewer et al. (2009b) und (2010).

16 Vgl. www.humanrights-centre.fau.de/index.shtml [10.12.2015]. 


\section{Danksagung}

Wir möchten allen Kolleginnen und Kollegen im EFI-Projekt »Human Rights in Healthcare « für die sehr gute Zusammenarbeit herzlich danken: PD phil. Lutz Bergemann (Wiss. Mitarbeiter an der Professur für Ethik in der Medizin, EFI-Fellow), Prof. Kai-Uwe Eckardt (Lehrstuhl für Innere Medizin IV), Prof. Yesim Erim (Psychosomatische und Psychotherapeutische Abteilung, Universitätsklinikum Erlangen), cand. med. Sonja Gaag (Doktorandin in der Psychosomatik), Prof. Elmar Gräßel (Medizinische Psychologie und Medizinische Soziologie, UK Erlangen), Prof. Christian Jäger (Lehrstuhl für Strafrecht, Strafprozessrecht, Wirtschafts- und Medizinstrafrecht), Dipl.-Pol. Sabine Klotz (Wiss. Mitarbeiterin am Lehrstuhl für Menschenrechte und Menschenrechtspolitik), Prof. Peter KolominskyRabas (Interdisziplinäres Zentrum für Public Health), Prof. Markus Krajewski (Lehrstuhl für Öffentliches Recht und Völkerrecht), PD Michael Krennerich (Lehrstuhl für Menschenrechte und Menschenrechtspolitik), Imke Leicht, M.A. (Betreuerin des Masterstudiengang »Human Rights«), Dilbar Mammadova (Ärztin an der Kinder- und Jugendklinik), Dr. PH Maren Mylius, M.A. (Stipendiatin an der Professur für Ethik in der Medizin), Prof. Christoph Ostgathe (Abteilung für Palliativmedizin in der Anästhesiologischen Klinik), Prof. Wolfgang Rascher (Kinderheilkunde, UK Erlangen), Dipl.-Sozialwirtin Sandra Schaller (Wiss. Mitarbeiterin am Interdisziplinären Zentrum für Public Health), Dipl.-Psych. Katharina Schieber (Wiss. Mitarbeiterin an der Psychosomatischen und Psychotherapeutischen Abteilung), Dr. Martina Schmidhuber (Wiss. Mitarbeiterin an der Professur für Ethik in der Medizin, EFI-Fellow), PD Caroline Welsh (Department Germanistik und Komparatistik). Ein ganz besonderer Dank geht an die beteiligten Kolleginnen in den jeweiligen Sekretariaten: Silvia Krönig (Menschenrechte und Menschenrechtspolitik), Anja Koberg, M.A. und Frauke Scheller, M.A. (Ethik in der Medizin).

Ohne die Förderung der FAU und die Emerging Fields Initiative wäre diese Kooperation nicht möglich gewesen. Herzlicher Dank geht an Prof. Joachim Hornegger, den Präsidenten der FAU, sowie die Vizepräsidentin für Forschung, Prof. Nadine Gatzert, zusammen mit dem EFI-Office. Dem transcript Verlag - insbesondere Julia Wieczorek, Michael Volkmer und Kai Reinhardt - danken wir ebenfalls für die sehr gute und reibungslose Zusammenarbeit. 


\section{LITERATUR}

Amnesty International. Sektion der Bundesrepublik e.V. (1996): »Diagnose: akutes Herzversagen «. Heilberufler und Menschenrechtsverletzungen. Gratwanderung zwischen Gewissen, Gefährdung und Komplizenschaft. Bonn: AI.

Bielefeldt, Heiner (1998): Philosophie der Menschenrechte. Grundlagen eines weltweiten Freiheitsethos. Darmstadt: Wissenschaftliche Buchgesellschaft.

Bielefeldt, Heiner (2009a): Zum Innovationspotenzial der UN-Behindertenrechtskonvention. Essay Nr. 5 des Deutschen Instituts für Menschenrechte. 3. Auflage. Berlin: DIMR.

Bielefeldt, Heiner (2009b): »Würde, Recht und Folter. Ein Menschenrechtsprinzip in der Krise?«, in: Frewer et al. (2009), 163-174.

Bielefeldt, Heiner (2011): Auslaufmodell Menschenwürde? Warum sie in Frage steht und warum wir sie verteidigen müssen. Herder: Freiburg.

Bielefeldt, Heiner/Ghanea, Nazila/Wiener, Michael (2016): Freedom of Religion or Belief. An International Law Commentary, Oxford: Oxford University Press.

Committee on Economic, Social, and Cultural Rights (2000): General Comment 14: The right to the highest attainable standard of health. E/C.12/2000/4 (August 11, 2000). www2.ohchr.org/english/bodies/ cescr/comments.htm [10.12.2015].

Council of Europe (1950): Convention for the Protection of Human Rights and Fundamental Freedoms. www.conventions.coe.int/Treaty/en/Treaties/Html/ 005.htm [10.12.2015].

Farmer, Paul (2005): Pathologies of Power. Health, Human Rights, and the War on the Poor. Berkeley: University of California Press.

Frewer, Andreas (2010): »Human rights from the Nuremberg Doctors Trial to the Geneva Declaration«, in: Medicine, Health Care and Philosophy 13 (2010), 259-268.

Frewer, Andreas/Furtmayr, Holger/Krása, Kerstin/Wenzel, Thomas (Hg.) (2009a): Istanbul-Protokoll. Untersuchung und Dokumentation von Folter und Menschenrechtsverletzungen. Medizin und Menschenrechte, Bd. 2. Göttingen: V \& R unipress. 
Frewer, Andreas/Kolb, Stephan/Krása, Kerstin (Hg.) (2009b): Medizin, Ethik und Menschenrechte. Geschichte - Grundlagen - Praxis. Medizin und Menschenrechte, Bd. 1. Göttingen: V \& R unipress.

Grover, Anand (2011): Thematic study on the realization of the right to health of older persons by the Special Rapporteur on the right of everyone to the enjoyment of the highest attainable standard of physical and mental health, Geneva, 4 July 2011, A/HRC/18/37.

Gruskin, Sofia/Grodin, Michael/Annas, George/Marks, Steven (Eds.) (2005): Perspectives on Health and Human Rights. New York: Routledge.

Krajewski, Markus (2010): »Grenzüberschreitende Patientenmobilität in Europa zwischen negativer und positiver Integration der Gesundheitssysteme«, in: Europarecht (EuR) 2 (2010), 165-188.

London, Leslie (2008): »What is a human rights-based approach to health and does it matter?«, in: Health and Human Rights 10, 1 (2008). www.hhrjournal.org/index.php/hhr/article/view/25/84 [10.12.2015].

Mann, Jonathan/Gruskin, Sofia/Grodin, Michael/Annas, George (Eds.) (1999): Health and Human Rights: A Reader. New York: Routledge.

Mylius, Maren/Bornschlegl, Wiebke/Frewer, Andreas (Hg.) (2011): Medizin für »Menschen ohne Papiere«. Menschenrechte und Ethik in der Praxis des Gesundheitssystems. Medizin und Menschenrechte, Bd. 5. Göttingen: V \& R unipress.

Ooms, Gorik/Latif, Laila A./Waris, Attiya/Brolan, Claire E/Hammonds, Rachel/Friedman, Eric A./Mulumba, Moses/Forman, Lisa (2014): »Is universal health coverage the practical expression of the right to health care? «, in: BMC International Health and Human Rights 14, 3 (2014), www.biomedcentral.com/1472-698X/14/3 [10.11.2015].

Rothhaar, Markus/Frewer, Andreas (Hg.) (2012): Das Gesunde, das Kranke und die Medizinethik. Moralische Implikationen des Krankheitsbegriffs, Stuttgart: Steiner .

Toebes, Brigit (Ed.) (2014): The Right to Health. A Multi-Country Study of Law, Policy and Practice, The Hague: T.M.C. Asser Press.

Toebes, Brigit C. A./Hartlev, Mette/Hendriks, Aart/Herrmann, Janne Rothmar (Eds.) (2012): Health and human rights in Europe. Cambridge u.a.: Intersentia.

UN Development Group (UNDG) (2003): The Human Rights Based Approach to Development Cooperation Towards a Common Understand- 
ing Among UN Agencies (May 2003). www.undg.org/archive_ docs/6959-The_Human_Rights_Based_Approach_to_Development_ Cooperation_Towards_a_Common_Understanding_among_UN.pdf.

UN General Assembly (1966): International Covenant on Civil and Political Rights, A/RES/21/2200. www.ohchr.org/EN/Professional Interest/ Pages/CCPR.aspx [10.12.2015].

World Health Organization (WHO) (2002): »25 Questions and Answers on Health and Human Rights", in: Health and Human Rights Publication Series 1 (2002). www.who.int/hhr/information/25_questions_hhr.pdf [10.12. 2015].

Yamin, Alicia (2008): »Beyond compassion: The central role of accounttability in applying a human rights framework to health.«, in: Health and Human Rights 10, 2 (2008). www.hhrjournal.org/index.php/hhr/article/ view/78/163 [10.12.2015]. 


\section{Der Menschenrechtsansatz im Gesundheitswesen}

Einige Grundsatzüberlegungen

HEINER BIELEFELDT

\section{KLÄRUNGSBEDARF}

Anlässlich einer Feier zum 60. Jahrestag der Allgemeinen Erklärung der Menschenrechte äußerte Morten Kjaerum, damaliger Direktor der EUGrundrechteagentur, den dringenden Wunsch, in Zukunft auch einmal die menschenrechtlichen Leistungen von Krankenschwestern oder Krankenpflegern (er verwendete den geschlechtsneutralen Begriff »nurses«) öffentlich gewürdigt zu sehen. Konkret schlug er vor, man möge dies doch spätestens bei den Vorbereitungen für den 70. Geburtstag der Allgemeinen Erklärung im Jahre 2018 einplanen. ${ }^{1}$ Hinter diesem Plädoyer verbirgt sich Kritik an einer etwas einseitigen menschenrechtlichen Ikonographie, bei der nach wie vor Bilder von friedlichen Demonstranten im Gegenüber zu einer martialischen Staatsmacht dominieren. Nicht dass diese Bilder falsch oder anachronistisch wären; sie haben leider an Aktualität nichts eingebüßt. Die menschenrechtliche Erschließung von Erfahrungsräumen und Lebensbereichen, die bis vor einigen Jahrzehnten als eher »unpolitisch « galten einschließlich des Gesundheitswesens -, ist aber weder im allgemeinen Be-

1 So Kjaerum auf einer Kooperationsveranstaltung des Nürnberger Menschenrechtszentrums zusammen mit der Stiftung »Erinnerung - Verantwortung Zukunft« in Nürnberg im Dezember 2008. Vgl. auch Kjaerum (2009), 19-24. 
wusstsein noch auch in den Bildern und Metaphern, in denen Menschenrechte typischerweise dargestellt werden, wirklich angekommen.

Dass engagierte Krankenschwestern und Pfleger als Menschenrechtler gewürdigt werden sollten, wie von Morten Kjaerum vorgeschlagen, dürfte für die meisten Menschen ungewohnt klingen. Zu den Berufsgruppen, die einem im Kontext von Menschenrechtsverteidigung spontan in den Sinn kommen, gehören Rechtsanwälte und Journalisten, vielleicht auch politisch engagierte Pfarrer - kaum aber Angehörige medizinischer Berufe oder gar Altenpflegerinnen. Dabei hatte die UN-Sonderberichterstattung zu »Human Rights Defenders« wiederholt auf die Bedeutung hingewiesen, die Ärzten, Krankenschwestern und Pflegern innerhalb ihres UN-Mandats zukommt. ${ }^{2}$

Wer eine Verbindung zwischen Menschenrechten und dem Gesundheitswesen herstellt, kann damit auch heute noch Überraschungen auslösen. In den von Erziehungswissenschaftlern regelmäßig durchgeführten Befragungen zum Wissensstand der deutschen Bevölkerung in Sachen Menschenrechte kommt das Recht auf Gesundheit praktisch nicht vor. ${ }^{3}$ Die Themen, die den Befragten einfallen, beziehen sich auf Internet-Zensur, Panzerlieferungen, Folter, willkürliche Verhaftungen und Schikanierung von Minderheiten - kaum je aber auf den Umgang mit Demenzpatienten, die medizinische Versorgung von Migranten ohne Papiere oder Ressourcenkonflikte bei der Organtransplantation.

Das Wissensdefizit, ja mehr noch: Wahrnehmungsdefizit, das sich hier zeigt, ist einerseits erstaunlich, andererseits vielleicht aber doch nicht völlig überraschend. Zunächst erstaunt es schon, dass selbst menschenrechtlich Interessierte und Engagierte von einem »Recht auf Gesundheit« meist noch nie gehört haben. Auch Ärzte kennen dieses Recht oftmals nicht. Damit konfrontiert, haben sie vielfach Schwierigkeiten, sich darunter etwas Sinnvolles vorzustellen. Dabei ist das Recht auf Gesundheit - zumindest in rudimentärer Form - bereits in der Allgemeinen Erklärung der Menschenrechte von 1948, dem Mutterdokument des internationalen Menschen-

2 Die thematischen und länderspezifischen Berichte des UN-Sonderberichterstatters für »Human Rights Defenders« und seiner Vorgängerinnen finden sich auf der folgenden Website des UN-Hochkommissariats für Menschenrechte: www.defenders.ohchr.org [11.01.2016].

3 Vgl. Stellmacher et al. (2007), 267-292. 
rechtsschutzes, enthalten. ${ }^{4}$ Es wurde im Internationalen Pakt über wirtschaftliche, soziale und kulturelle Rechte von 1966 völkerrechtlich verbindlich ausgestaltet ${ }^{5}$ und in einem »General Comment « des dafür zuständigen UN-Ausschusses im Jahre 2000 erläutert und ausdifferenziert. ${ }^{6}$ Die aus den Perspektiven spezifischer Betroffenheit formulierten Konventionen zur Abschaffung aller Formen der Diskriminierung der Frau, zu den Rechten des Kindes, zu den Rechten der Wanderarbeitnehmer und ihrer Familienangehörigen sowie zu den Rechten von Menschen mit Behinderungen enthalten allesamt Garantien für den Bereich des Gesundheitswesens. ${ }^{7}$ Seit 2002 gibt es außerdem das Amt des UN-Sonderberichterstatters für das Recht auf Gesundheit. ${ }^{8}$ Innerhalb des Europarats enthält die Europäische Sozialcharta in ihrer revidierten Fassung von 1996 das Recht auf Gesundheit. ${ }^{9}$ Die EUGrundrechtecharta, in Kraft getreten im Dezember 2009 im Rahmen des Lissabonner Vertrags, enthält dieses Recht ebenfalls. ${ }^{10}$ Nicht zuletzt findet sich das Recht auf Gesundheit in einer Reihe nationaler Verfassungen, und Gerichte in vielen Ländern der Welt beschäftigen sich anhand konkreter Streitfälle mit seiner Anwendung in der Praxis. ${ }^{11}$

4 Vgl. Artikel 25 Absatz 1 der Allgemeinen Erklärung der Menschenrechte.

5 Vgl. Artikel 12 des Internationalen Pakts über wirtschaftliche, soziale und kulturelle Rechte.

6 Vgl. CESCR E/C.12/2000/4, 11. August 2000. Eine deutsche Übersetzung findet sich in Deutsches Institut für Menschenrechte (2005), 285-313 und in diesem Band.

7 Vgl. Artikel 12 des Abkommens zur Abschaffung aller Formen der Diskriminierung der Frau (1979); Artikel 24 der Konvention über die Rechte des Kindes (1989); Artikel 28 der Konvention zum Schutz der Rechte aller Wanderarbeitnehmer und ihrer Familienangehörigen (1990); Artikel 25 der Konvention über die Rechte von Personen mit Behinderungen (2006).

8 Der erste Inhaber dieses Amts war Paul Hunt, dem 2008 Anand Grover folgte. Seit 2014 obliegt die Funktion dem litauischen Arzt Dainius Pūras. Die thematischen und länderbezogenen Berichte der Sonderberichterstatter sind auf der Website des UN-Hochkommissariats für Menschenrechte verfügbar: www. srhealth.ohchr.org.

9 Vgl. Artikel 11 der Europäischen Sozialcharta, revidierte Fassung von 1996.

10 Vgl. Artikel 35 der EU-Grundrechtecharta.

11 Vgl. Krennerich (2013), 203-227. 
Das gleichwohl bestehende Wahrnehmungsdefizit könnte u.a. daher rühren, dass die übliche menschenrechtliche Empowerment-Semantik im Gesundheitswesen gelegentlich merkwürdig deplatziert wirkt. In der Konfrontation mit schwerer Krankheit, Behinderung oder bevorstehendem Tod stoßen Menschen an Grenzen, die unverrückbar sein können. Muss es dann nicht vor allem darum gehen, die eigene Endlichkeit, ja letzte Ohnmacht zu akzeptieren? Müssen Menschen in solchen Situationen nicht lernen, loszulassen und sich in andere Hände zu begeben? Brauchen sie nicht eher Zuwendung und Fürsorge - vielleicht auch Seelsorge - als die Zuerkennung von Rechtspositionen in Verbindung mit effizienter Rechtsdurchsetzung? Sollten wir nicht offen einräumen, dass zur conditio humana Leidenserfahrungen sowie die Dimension des Tragischen gehören, die mit der menschenrechtlichen Empowerment-Semantik partout nicht zusammenpassen? Wäre es dann aber nicht ratsam, Zurückhaltung zu wahren, statt das Gesundheitswesen mit womöglich irreführenden menschenrechtlichen Ansprüchen zu traktieren? Noch einmal grundsätzlicher gefragt: Kann es einen Rechtsanspruch auf Gesundheit im eigentlichen Sinne überhaupt geben, und wer soll ihn garantieren?

An solchen Fragen erweist sich die Notwendigkeit von Klärungen. Dabei geht es nicht nur darum, Missverständnisse - darunter etwaige »prometheische« Überziehungen in menschenrechtlicher Semantik und Metaphorik - auszuräumen, sondern präzise interdisziplinäre Übersetzungsprozesse zu leisten. Dies birgt zugleich Chancen auf vertiefte Einsichten für alle Beteiligten. Auseinandersetzungen um Menschenrechte im Gesundheitswesen eröffnen neue Perspektiven nicht nur für Mediziner, wenn sie ihre Praxis systematisch im Lichte menschenrechtlicher Prinzipien und Normen betrachten, sondern auch für Menschenrechtler, die in der Auseinandersetzung mit medizinethischen Grenzfragen menschlicher Existenz Anspruch, Reichweite und Grenzen des Menschenrechtsansatzes aufs Neue reflektieren müssen.

Der vorliegende Aufsatz kann dazu nur einige knappe Grundsatzüberlegungen aus der Sicht der Menschenrechte vorbringen. Bei der Strukturierung des Textes orientiere ich mich an den andernorts ausführlicher erläuterten Prinzipien des Menschenrechtsansatzes: ${ }^{12}$ dem normativen Universalismus der Menschenrechte (Kap. 2), ihrer um den Begriff der Autonomie

12 Vgl. Bielefeldt (1998). 
zentrierten freiheitlichen Orientierung (Kap. 3), dem Grundsatz der Gleichheit, spezifiziert in Diskriminierungsverboten (Kap. 4) und der menschenrechtlichen Garantenfunktion des Staates (Kap. 5). Zusammen genommen definieren diese Komponenten die Menschenrechte als in der Menschenwürde jedes Menschen gegründete universale Freiheits- und Gleichheitsrechte, für die der Staat spezifische Gewährleistungsfunktionen übernommen hat. Der Text mündet in einige abschließende Bemerkungen (Kap. 6).

Bevor ich in die derart strukturierte Grundsatzreflexion einsteige, sei kurz darauf hingewiesen, dass das soeben paradigmatisch angeführte Recht auf Gesundheit keineswegs das einzige für den Kontext des Gesundheitswesens einschlägige Menschenrecht darstellt. Vielmehr steht dieses Recht im Zusammenhang auch anderer Menschenrechte, etwa der Rechte auf Bildung, soziale Sicherheit, Schutz der Privatsphäre, Respekt religiöser Sensibilitäten, Kontakt mit Familienangehörigen, Information und Kommunikation, Zugang zu Rechtsmitteln für Fälle etwaiger Rechtsverletzung usw. Die Menschenrechte bilden einen komplexen normativen Gesamtzusammenhang, in dem die einzelnen Rechte einander wechselseitig voraussetzen und bedingen. ${ }^{13}$

Die Subjekte dieser und anderer Rechte im Kontext des Gesundheitswesens sind im Übrigen keineswegs nur Patientinnen und Patienten, an die man vermutlich zunächst denkt, sondern beispielsweise auch Ärztinnen und Ärzte sowie andere im Gesundheitswesen tätige Personen. ${ }^{14}$ Erinnert sei etwa an ihre Rechte auf freie gewerkschaftliche Betätigung, auf gleichberechtigte Berufs- und Aufstiegschancen für Frauen und Männer innerhalb der Krankenhaushierarchie und auf regelmäßige Erholung und Freizeit. Ein sensibles, in der Öffentlichkeit eher wenig beachtetes Feld betrifft die gewissensbedingte Weigerung, an bestimmten Maßnahmen - etwa Abtreibungen - mitzuwirken, wenn sie den eigenen religiösen oder moralischen Überzeugungen zuwiderlaufen; dies fällt in den Bereich der Gewissens-,

13 Dafür hat sich der Begriff der Unteilbarkeit der Menschenrechte durchgesetzt, der prominent im Abschlussdokument der Wiener Weltmenschenrechtskonferenz 1993 firmiert: »All human rights are universal, indivisible and interdependent and interrelated.« World Conference on Human Rights (1993). Vienna Declaration and Programme of Action, Nr. 5, erster Satz.

14 Vgl. London (2008). 
Religions- und Weltanschauungsfreiheit, die auch in der professionellen Praxis des Gesundheitswesens beachtet werden muss. ${ }^{15}$

Man könnte noch viele weitere Beispiele anführen. Kurz: Das Panorama der für das Gesundheitswesen relevanten Menschenrechte ist weit gespannt und umfasst die Rechte nicht nur von Patienten, sondern letztlich von allen in diesem Feld tätigen und betroffenen Menschen.

\section{DeR UniVersalismus VON MENSChENWÜRDE UND MENSCHENRECHTEN}

»Menschenrechte sind universal - oder sie sind nicht. ${ }^{16}$ Mit diesen knappen Worten unterstreicht Udo di Fabio die zentrale Relevanz des normativen Universalismus, der den Anspruch der Menschenrechte im Ganzen definiert. Die Begriffe Menschenrechte und Universalismus sind sachlich so miteinander verklammert, dass die Rede von universalen Menschenrechten fast schon wie ein Pleonasmus wirkt. Und dennoch (oder auch gerade deshalb) sorgt der Universalismus der Menschenrechte seit jeher für Kontroversen. Manche Verwirrung rührt daher, dass der Begriff des Universalismus oft wie eine quasi-geographische Kategorie gehandhabt wird. Er bezeichnet dann nicht so sehr die innere Normstruktur der Menschenrechte als vielmehr die territoriale Reichweite ihres Geltungsanspruchs. Vor allem im juristischen Sprachgebrauch ist es üblich, die »universalen« Menschenrechte vornehmlich mit den globalen Standards der Vereinten Nationen zu assoziieren und gegenüber regionalen oder nationalen Rechtsebenen abzugrenzen. ${ }^{17}$ In solch quasi-räumlicher Kontrastierung zu sub-globalen (regionalen oder nationalen) Rechtsverbürgungen repräsentieren die universalen Menschenrechte scheinbar ein Phänomen politisch-rechtlicher Globalisie-

15 Vgl. Bielefeldt et al. (2016), 258-308.

16 Di Fabio (2008), 63. Di Fabio war von 1999 bis 2011 Richter am Bundesverfassungsgericht.

17 Eine einseitige Fokussierung auf die Ebene der Vereinten Nationen könnte aber einem Missverständnis Nahrung geben, dem schon Hannah Arendt erlegen war, nämlich dass die Menschenrechte nur gleichsam die äußerste, »abstrakteste« Sphäre menschheitsweiter Verbindlichkeiten repräsentieren. Vgl. Arendt (1949) und Arendt (1974), 452-470. 
rung, analog zu anderen Aspekten von Globalisierung, etwa in der Ökonomie oder in der Kultur. Die räumlich-globalen Wirkungen, die der Menschenrechtsansatz tatsächlich entfaltet, sind allerdings eher eine (wichtige) Konsequenz des normativen Universalismus und machen nicht schon dessen Kern aus. Es ist ja kein Zufall, dass wir auch im Kontext des Europarats von "Menschenrechten« sprechen, obwohl die Europäische Menschenrechtskonvention und der auf ihrer Grundlage arbeitende Europäische Gerichtshof für Menschenrechte gerade keine weltumspannende territoriale Geltung beanspruchen. Auch diejenigen Rechte, die im Grundrechtsabschnitt des deutschen Grundgesetzes für alle innerhalb des Jurisdiktionsbereichs der Bundesrepublik Deutschland lebenden Menschen (also nicht nur für Staatsangehörige) gelten, werden bekanntlich »Menschenrechte« genannt. Unter Menschenrechten verstehen wir demnach keineswegs nur die auf der Weltbühne in New York oder Genf normierten globalen Standards, sondern auch Rechtsnormen, deren tragende Institutionen uns geographisch sehr viel näher liegen.

Die primäre Bedeutung des Begriffs Universalismus für das Verständnis der Menschenrechte besteht darin, dass er eine Kategorie fundamentaler Rechte auszeichnet, die dem Menschen nicht erst aufgrund partikularer Rollen, Funktionen, Vorleistungen, Dokumente, Mitgliedschaften usw. zugesprochen werden, sondern die ihm schon allein aufgrund seines Menschseins zukommen. Menschenrechte sind die Grundrechte des Menschen und zwar jedes Menschen -, und genau darin besteht ihre universalistische Struktur. ${ }^{18}$ Der Universalismus der Menschenrechte steht, so gesehen, nicht primär im Kontrast zu regionalen oder nationalen Rechtsordnungen, sondern bildet vornehmlich den Gegenbegriff zu einem rechtlichen Partikularismus, der bei je besonderen Statuspositionen oder Mitgliedschaften stehenbleibt, die eben nicht jedem Menschen zukommen. Weil im Menschsein des Menschen und letztlich in der Würde jedes Menschen begründet, beanspruchen die universalen Menschenrechte einen normativen Vorrang gegenüber partikularen Rechtspositionen. ${ }^{19}$

Nach wie vor sind die meisten Rechte, von denen wir alltäglich Gebrauch machen, an partikulare Rollen, Funktionen, Mitgliedschaften oder Vorleistungen gebunden. Hier einige Beispiele: Menschen besitzen etwa

18 Vgl. Reuter (1999).

19 Vgl. Schwartländer (1978). 
Rechtsansprüche als Mitglieder einer Krankenversicherung, für die sie finanzielle Leistungen erbringen. Wer das Recht erwerben will, einen Doktortitel zu tragen, muss dafür eine Dissertation verfassen und Prüfungen erfolgreich absolvieren. Menschen üben Mitwirkungsrechte in einer Partei oder einer Berufsvereinigung aus, der sie sich verbunden fühlen und für die sie regelmäßig Beiträge entrichten. In einem Mietvertrag werden die wechselseitigen Rechte und Pflichten von Mietern und Vermietern ausgehandelt - einschließlich der Modalitäten einer möglichen Kündigung. Manche Rechte, etwa Copyrights an eigenen Aufsätzen, lassen sich auch gegen Geld verkaufen. Generell gilt, dass wir unsere vielfältigen partikularen Rechte erwerben und aufgeben, kaufen und verkaufen oder kündigen können; sie können uns auch verliehen oder abgesprochen werden - abhängig von Rollen und Funktionen, die wir wahrnehmen, oder von Leistungen, die wir erbringen bzw. nicht erbringen. Bei universalen Rechten ist dies ganz anders. Weil im Menschsein des Menschen begründet, können sie nicht zugesprochen oder abgesprochen, erworben, gekündigt oder veräußert werden. Menschenrechte bilden insofern eine andere Kategorie von Rechten. Weil an das Menschsein des Menschen gebunden, haben sie den herausgehobenen Rang »unveräußerlicher Rechte«, ${ }^{20}$ wie es schon in den Grunddokumenten der Menschenrechte heißt. Die einzige »Mitgliedschaft«, die in diesem Zusammenhang zählt, ist die Mitgliedschaft in der Menschheitsgemeinschaft, die bei jedem Menschen unterstellt werden muss.

Das Verhältnis von universalen und partikularen Rechten bildet kein Nebeneinander, sondern manifestiert sich als klares Vorrangverhältnis. ${ }^{21}$ Das heißt gewiss nicht, dass »erworbene« partikulare Rechtspositionen unter dem vorrangigen Geltungsanspruch der universalen Menschenrechte einfach dahinschmelzen; wohl aber werden sie unter einschränkende Be-

20 Dieser Begriff kommt etwa in der von der Philosophie John Lockes inspirierten Amerikanischen Unabhängigkeitserklärung vor. Er findet sich auch in der Präambel der Allgemeinen Erklärung der Menschenrechte sowie in Artikel 1 Absatz 2 des Grundgesetzes der Bundesrepublik Deutschland.

21 Um Missverständnisse zu vermeiden, muss zwischen Partikularität und »Spezifizität« unterschieden werden. Der Vorrang der universalen Menschenrechte spricht nicht dagegen, auf der Ebene positivierter Rechte den spezifischen Regeln Priorität gegenüber unspezifischen - d.h. »allgemeinen « im Sinne von »generellen« - Regeln einzuräumen. 
dingungen gestellt und dadurch ggf. auch inhaltlich transformiert. Dazu einige Beispiele aus dem Bereich des Gesundheitswesens.

Aufgrund seines Ranges als eines universalen Menschenrechts kommt dem Recht auf Gesundheit ein systematischer Vorrang gegenüber denjenigen partikularen Rechtsansprüchen zu, die ein Mensch aufgrund seiner Mitgliedschaft in einer Krankenversicherung besitzt, denn das Menschsein des Menschen hat einen anderen normativen Rang als der partikulare Status eines Kunden. Im Ergebnis läuft dies natürlich nicht auf die Abschaffung der Krankenversicherungen oder auf die Beseitigung sämtlicher marktwirtschaftlicher Elemente im Gesundheitswesen hinaus. Vielmehr verlangt das Menschenrecht auf Gesundheit Maßnahmen, die sicherstellen, dass Menschen, die aufgrund ihrer geringen Marktmacht keine »attraktiven Kunden« für das Gesundheitssystem sind, gleichwohl angemessenen Zugang zur Gesundheitsversorgung haben. ${ }^{22}$ Wie dies näherhin geschehen soll, wird nicht im Einzelnen normativ vorgegeben. Hier können unterschiedliche Gesellschaften ihre je eigenen Wege beschreiten und über wirksame Maßnahmen kontrovers streiten oder auch neue Maßnahmen ausprobieren. Aus guten Gründen enthalten Menschenrechte keine umfassenden Blaupausen für die konkrete Funktionsweise gesellschaftlicher Subsysteme. Wichtig ist aber, dass der Zugang zu Gesundheitsleistungen nicht als bloß caritative Hilfe gewährt, sondern als verlässlicher Rechtsanspruch eines jeden ausgestaltet wird. Universalismus heißt in diesem Kontext, dass niemand aufgrund geringer Finanzmöglichkeiten in die Situation geraten soll, um wichtige medizinische Unterstützung betteln zu müssen.

Ein anderes Beispiel betrifft Migrantinnen und Migranten ohne Papiere, also Menschen, deren Aufenthalt ordnungsrechtlich als »illegal « gilt. ${ }^{23}$ Der Vorrang des Menschenrechts auf Gesundheit bedeutet hier, dass aufenthaltsrechtliche Statusfragen - trotz ihrer unbestrittenen ordnungsrechtlichen Relevanz für den Staat - kein Grund für den Ausschluss aus der Gesundheitsversorgung sein dürfen. Ob ein Mensch Staatsangehöriger ist oder nicht, ob er über einen legitimen Aufenthaltstitel verfügt oder nicht - das Recht auf Gesundheit kommt ihm vorgängig und vorrangig zu allen parti-

22 Vgl. CESCR E/C.12/2000/4, 11. August 2000, Ziff. 19: »Die Staaten haben eine besondere Verpflichtung, jenen, die nicht über ausreichende Mittel verfügen, Krankenversicherung und Gesundheitsfürsorge zur Verfügung zu stellen [...].«

23 Zum Folgenden vgl. Mylius et al. (2011). 
kularen Statuspositionen zu; genau darin zeigt sich wiederum der normative Universalismus. Wie der Anspruch auf Zugang zum Gesundheitssystem für Menschen ohne Papiere wirksam zur Geltung kommen kann, ist politisch in Deutschland nach wie vor umstritten. Die in diesem Feld engagierten Menschenrechtsorganisationen weisen darauf hin, dass faktisch nach wie vor allerlei administrative Stolperdrähte existieren, die eine angstfreie Wahrnehmung des Rechts auf Gesundheit für Menschen ohne Papiere erheblich behindern. Der Vorrang des universalen Menschenrechts vor partikularen rechtlichen Statuspositionen kommt in der Praxis offenbar keineswegs konsequent zum Zuge. Hier besteht nach wie vor Reformbedarf.

Besonders schwierige Fragen stellen sich dort, wo wichtige Ressourcen so knapp sind, dass manche Menschen im Ergebnis leer ausgehen werden, wenn beispielsweise im Kontext der Transplantationsmedizin nicht genügend Spenderorgane vorliegen, um alle darauf angewiesenen Patientinnen und Patienten zu versorgen. Das Recht auf Gesundheit enthält nicht das Versprechen, dass ein jeder stets das erhalten kann, was er braucht; Universalismus ist keine utopische Kategorie. Die schwierigen, ja tragischen Entscheidungen, die in manchen Fällen unvermeidlich sind, müssen freilich so getroffen werden, dass die dabei zugrunde gelegten Kriterien hypothetisch gegenüber allen Betroffenen plausibel gemacht werden könnten. Auch diejenigen, die im Ergebnis leer ausgehen, haben ein Recht auf Rechtfertigung $^{24}$ einer für sie nachteiligen Entscheidung. Dass sie einer solchen Entscheidung tatsächlich zustimmen werden, kann man kaum erwarten, denn dies würde ein geradezu heroisches Menschenbild voraussetzen. Unter dem Anspruch des menschenrechtlichen Universalismus besteht gleichwohl die Aufgabe, Entscheidungen systematisch und nachvollziehbar an solchen Kriterien zu orientieren, die sich - zumindest hypothetisch - gegenüber allen Betroffenen plausibel darlegen lassen könnten.

Der letzte, tragende Grund des normativen Universalismus besteht im gebotenen Respekt vor der Würde eines jeden Menschen. ${ }^{25}$ Nicht nur gemäß Artikel 1 des Grundgesetzes, auch gemäß der - ein halbes Jahr früher verabschiedeten - Allgemeinen Erklärung der Menschenrechte kommt der Menschenwürde ein axiomatischer Stellenwert für die Rechtsordnung im

24 Vgl. Forst (2007).

25 Zum Folgenden Näheres in Bielefeldt (2011). 
Ganzen zu. ${ }^{26}$ Zwischenmenschliche Verbindlichkeiten jedweder Art setzen die Achtung der Würde jedes Menschen immer schon voraus. In diesem Sinne beginnt die Präambel der UN-Erklärung von 1948 mit der Einsicht, dass »die Anerkennung der allen Mitgliedern der menschlichen Familie inhärenten Würde und ihrer gleichen und unveräußerlichen Rechte« die Grundlage jedweden Rechts darstellt. ${ }^{27}$

Die Anerkennung (»recognition«) der Würde ist gleichsam das erste Wort im ersten Satz der Präambel des ersten internationalen Menschenrechtsdokuments. »Anerkennung « ist hier nicht sozialkontraktualistisch im Sinne einer wechselseitigen Zusage zu verstehen, durch die die Würde der Menschen erst gleichsam konstituiert werden würde; vielmehr handelt es sich um eine Grundeinsicht in den axiomatischen Stellenwert jener Achtung, die dem Menschen als Menschen gebührt, die ihm als Mitglied der menschlichen Familie also immer schon »inhärent« ist, wie es im Text der Präambel weiter heißt. ${ }^{28}$ Die Anerkennung der Würde ist kein Geschäft auf Wechselseitigkeit, sondern resultiert aus der Einsicht in ihre notwendige Allseitigkeit. ${ }^{29}$ Die Menschenwürde ist das Fundament des menschenrechtlichen Universalismus.

Die UN-Behindertenrechtskonvention von 2006 verbindet die axiomatische Qualität des Begriffs der Menschenwürde mit einer sozialkritischen Verwendung des Würdebegriffs, wie sie auch im alltäglichen Sprachgebrauch vorkommt. In der Konvention geschieht dies über die Figur des »Würdebewusstseins « (»sense of dignity«), ${ }^{30}$ dessen Entwicklung wesentlich von den konkreten, empirischen Lebensumständen eines Menschen abhängt. Diese Figur des »sense of dignity« gehört zu den Neuerungen der Konvention; sie findet sich in anderen internationalen Menschenrechtsdokumenten bislang nicht. Dahinter stehen die Unrechtserfahrungen von

26 Vgl. dazu Morsink (1999).

27 Die offizielle deutsche Übersetzung weist leider erhebliche Schwächen auf. So wird »inherent dignity« mit »angeborener Würde« wiedergeben - was Fragen nach dem Status des ungeborenen Lebens aufwerfen könnte, für die das Original überhaupt keinen Anlass bietet. Die Metapher der »human family« verblasst im Deutschen zur »menschlichen Gemeinschaft«.

28 Vgl. Dicke (1992), 161-182.

29 Vgl. Spaemann (1996).

30 Vgl. Artikel 24 der UN-Behindertenrechtskonvention. 
Menschen mit Behinderungen, die teils erleben mussten, dass man sie vor der Öffentlichkeit versteckt und von der Gesellschaft absondert, als würde man sich ihrer Existenz geradezu »schämen«. Unter solchen Bedingungen dürfte es schwierig, ja fast unmöglich sein, ein Bewusstsein der eigenen Würde - und damit überhaupt ein Verständnis von Menschenwürde - zu entwickeln. Mit dem Begriff des »sense of dignity« schlägt die Behindertenrechtskonvention eine Brücke zwischen dem Bekenntnis zur Menschenwürde als axiomatischem Prinzip einerseits und der konkreten Lebenswelt der Menschen andererseits, die so gestaltet sein muss, dass diese tatsächlich die Möglichkeit haben, ein Bewusstsein ihrer eigenen Würde und der Würde anderer entwickeln zu können. Der Begriff der Menschenwürde bleibt damit in seiner axiomatischen Qualität ungeschmälert bestehen und kann gleichzeitig - über die Figur des "sense of dignity« - als Maßstab zur Kritik an konkreten empirischen Sozialverhältnissen dienen, die der Menschenwürde womöglich eben nicht gerecht werden.

Neben die axiomatische und die sozialkritische Verwendung tritt nicht selten ein weiterer Sprachgebrauch, der dadurch Verwirrung stiften kann, dass die Würde dabei in Abhängigkeit von persönlichen Leistungen des Individuums gerät. Die Semantik der »dignitas« weist nämlich von Anfang an eine eigentümliche Mehrdeutigkeit auf, ${ }^{31}$ insofern sie einerseits die gleiche Würde aller Menschen bezeichnet, andererseits historisch häufig auch mit Rangabstufungen - als Ergebnis hoher Geburt, bedeutender Ämter oder persönlicher Leistung - assoziiert worden ist. Schillers Abhandlung über »Anmut und Würde« bietet ein klassisches Beispiel für einen gestuften, leistungsorientierten Würdebegriff. ${ }^{32}$ Der Würde ist in Schillers Sprachgebrauch ein Element des Männlich-Heroischen eigen, durch das sie sich von der eher feminin konnotierten »Anmut« unterscheidet. Vor allem in persönlichen Bewährungssituationen erweist sich, ob es der Mensch schafft, seine Würde zu wahren. ${ }^{33}$ Wie für die Anmut gilt auch für die Würde, dass sie gelingen oder auch misslingen kann. Würde ist nach Schiller daher kei-

31 Vgl. Pöschl/Kondylis (1978), 637-677.

32 Vgl. Schiller (1954), 193-258.

33 Vgl. Schiller (1954), 244-245: »Bei der Würde also führt sich der Geist in dem Körper als Herrscher auf, denn hier hat er seine Selbständigkeit gegen den gebieterischen Trieb zu behaupten, der ohne ihn zu Handlungen schreitet, und sich seinem Joch gern entziehen möchte.« 
neswegs jedem gegeben. Dies ist der entscheidende Punkt. Wenn eine Bewährungsprobe für die Würde negativ ausgeht, so bedeutet dies folglich, dass der entsprechende Mensch seine Würde ganz oder teilweise einbüßt. Mit Universalismus hat dies offensichtlich nichts zu tun.

In der alltäglichen Rede von Würde und »würdigem Verhalten« finden sich Facetten eines solchen leistungsorientierten Verständnisses recht häufig. Ausgerechnet der Begriff der Würde kann daher auch als Einfallstor für partikularistische Relativierungen fungieren. Beobachten lässt sich dies etwa in den aktuellen Diskussionen über Sterbehilfe. Das Motto »Sterben in Würde« erweist sich dabei als zutiefst zweideutig. Auf der einen Seite kann es bedeuten, dass die unantastbare und unveräußerliche Würde des Menschen selbstverständlich auch innerhalb der Sterbephase zu respektieren ist. Menschenwürde ist in diesem Verständnis unauflöslich mit dem Menschsein des Menschen verknüpft, das kategorisch zu achten und zu schützen ist, solange der Mensch lebt - und das im Anspruch auf pietätvollen Umgang mit dem toten Körper noch über das Leben hinaus nachwirkt. Die Rede vom »Sterben in Würde« kann sich aber auch in Richtung eines Postulats verschieben, sich beizeiten einen »würdigen Abgang « zu verschaffen, bevor man in die missliche (»würdelose«) Lage gerät, vollends von der Fürsorge anderer abhängig zu werden. Hier nimmt der Sprachgebrauch unter der Hand partikularistische Züge an und mutiert zu einem Leistungsbegriff mit womöglich gar bedrohlichen Zügen für diejenigen, die weder Anmut noch Würde (im Schillerschen Sinne) zustande bringen können. Auch das Fundament der universalen Menschenrechte, die Menschenwürde eines jeden, bedarf offensichtlich stets kritischer Aufmerksamkeit, um es gegen semantische Verwechslungen zu schützen. Franz Müntefering hat dazu auf seine Art jüngst einen Beitrag geleistet. In einer Debatte zur Sterbehilfe vermerkte er trocken: »Die Würde des Menschen hat nichts zu tun damit, ob er sich selbst den Hintern abputzen kann. « ${ }^{34}$ Damit hat er den Universalismus von Menschenwürde und Menschenrechten knapp, aber treffend auf den Punkt gebracht.

34 Müntefering (2014). 


\section{Autonomie und fReie Selbstbestimmung}

Wie die Menschenwürde philosophisch oder theologisch näher zu verstehen ist, wird in den internationalen Menschenrechtsdokumenten nicht definiert. ${ }^{35}$ Diese Zurückhaltung ist sinnvoll. Menschenrechte haben ihre Funktion darin, unverzichtbare praktische Konsequenzen der gebotenen Anerkennung der Menschenwürde - nämlich in Gestalt durchsetzbarer Rechte präzise zu formulieren, nicht eine bestimmte philosophische oder theologische Interpretation der Würde vorzugeben. Bei aller generellen Zurückhaltung enthalten die menschenrechtlichen Grunddokumente gleichwohl einige inhaltliche Hinweise zum Verständnis der Menschenwürde. So heißt es in Artikel 1 der Allgemeinen Erklärung der Menschenrechte, alle Menschen seien »mit Vernunft und Gewissen ausgestattet «. ${ }^{36}$ Diese Formel findet sich unmittelbar im Anschluss an den viel zitierten Satz, wonach alle Menschen »frei und an Würde und Rechten gleich « geboren sind.

Wie ist die Formel von der Ausstattung mit »Vernunft und Gewissen« zu verstehen? Als empirische Deskription ergibt der Satz wenig Sinn. Zumal als deskriptive All-Aussage (»alle Menschen«) wäre er reichlich kühn, und man wäre doch sehr versucht, das eine oder andere Gegenbeispiel anzuführen. Um einen präskriptiven Satz, also eine normative Vorgabe, kann es sich aber auch nicht handeln. Normative Appelle setzen Vernunft und Gewissen beim Adressaten in gewisser Weise schon voraus. Man kann niemanden ernsthaft moralisch dazu auffordern, sich als Moralsubjekt überhaupt erst zu konstituieren. Sinn ergibt die Formel von der Ausstattung eines jeden mit Vernunft und Gewissen nur als notwendige Zuschreibung seitens der Rechtsgemeinschaft. Es handelt sich weder um eine deskriptive Aussage noch um eine präskriptive Aufforderung, sondern um eine askriptive Stellungnahme der Rechtsgemeinschaft. Diese bekennt sich damit dazu, jedem Menschen den Status eines - zumindest potenziellen - Verantwortungssubjekts zuzuschreiben und ihn entsprechend zu behandeln. Dies folgt unmittelbar aus der Anerkennung der Würde jedes Menschen. ${ }^{37}$

35 Vgl. Vögele (1999), 103-133.

36 Die offizielle deutsche Übersetzung für »endowed« verwendet das Adjektiv »begabt«, wodurch das Missverständnis nahe gelegt wird, es handele sich um eine individuelle Begabung.

37 Vgl. Gewirth (1992), 10-46. 
Der fundamentale Stellenwert dieser notwendigen Zuschreibung zeigt sich darin, dass sie strikt allen Menschen gilt. Nichts wäre deshalb abwegiger, als die Begriffe Vernunft und Gewissen in der Allgemeinen Erklärung als Ausschlusskriterien zu verstehen und etwa geistig Behinderten ihre Menschenrechte abzusprechen. Es kann nicht darum gehen, dass bestimmte Menschen gleichsam erst einen Test zu durchlaufen hätten, ob sie denn ausreichend Vernunft und Gewissen vorweisen können, um als Subjekte von Menschenwürde und Menschenrechten zu gelten. Vielmehr besteht die Aufgabe darin, jeden Menschen so zu behandeln, dass sein Status als Verantwortungssubjekt - ggf. auch unter widrigen oder gar extremen Umständen - nach Maßgabe des Möglichen maximal zur Geltung kommen kann. Die Forderung, die in der Formel von der Ausstattung mit Vernunft und Gewissen steckt, richtet sich insofern nicht an den einzelnen Menschen, der hier etwas nachzuweisen hätte, sondern sie richtet sich an die Rechtsgemeinschaft im Ganzen. Diese ist gehalten, in jedem Menschen gleichsam das Verantwortungssubjekt zu »sehen«, das in manchen Grenzfällen faktisch gerade nicht - noch nicht, nicht mehr oder auch nie ganz - unmittelbar "sichtbar« präsent ist, und aus dieser gebotenen Sichtweise praktische Konsequenzen zu ziehen.

Die praktischen Konsequenzen dieser notwendigen Zuschreibung werden in den einzelnen Menschenrechten formuliert, die allesamt auf je spezifische Weise Freiheitsrechte sind. ${ }^{38}$ Die Achtung vor der Würde des Menschen als Verantwortungssubjekt manifestiert sich rechtlich in Gewährleistungen freier Selbstbestimmung (bzw. freier gemeinschaftlicher Mitbestimmung) in den durch die einzelnen Rechte jeweils spezifisch adressierten Lebensbereichen. Manchen Menschenrechten ist diese freiheitliche Orientierung gleichsam an die Stirn geheftet - man denke etwa an Gedankenfreiheit, Gewissensfreiheit, Religionsfreiheit, Meinungsfreiheit, Informationsfreiheit, Versammlungsfreiheit, Vereinigungsfreiheit, Berufsfreiheit usw. Bei anderen Rechten muss man etwas genauer hinschauen, um die freiheitliche Struktur zu erkennen. Zu den Justizrechten gehört beispielsweise die Komponente, sich einen Rechtsbeistand frei wählen zu können. Auch in der Konfrontation mit der Strafjustiz soll der Mensch sich als Rechtssubjekt behaupten können. Die wirtschaftlichen und sozialen Menschenrechte erweisen sich bei näherem Hinsehen übrigens ebenfalls als 
Freiheitsrechte. ${ }^{39}$ Es geht dabei nicht nur um Versorgungsansprüche, sondern immer zugleich um den Respekt, der dem Menschen um seiner Würde als Verantwortungssubjekt willen gebührt. Auch das Recht auf Gesundheit ist in diesem Sinne zu verstehen. Es ist kein bloßes Versorgungsrecht, sondern wesentlich ein Freiheitsrecht. Laut General Comment Nr. 14 des UNAusschusses für wirtschaftliche, soziale und kulturelle Rechte (WSK-Ausschuss) schließt dies

»das Recht ein, über die eigene Gesundheit und den eigenen Körper zu bestimmen, einschließlich der sexuellen und reproduktiven Freiheit, das Recht, frei von Eingriffen zu sein und das Recht, nicht misshandelt, nicht medizinischer Behandlung oder medizinischen Versuchen ohne Einwilligung unterzogen zu werden «. ${ }^{40}$

In der UN-Behindertenrechtskonvention vom Dezember 2006, einem der jüngsten internationalen Menschenrechtsinstrumente, kommt der Anspruch auf freie Selbstbestimmung besonders prononciert zum Tragen. Er manifestiert sich hier in einem Begriff, der in einigen philosophischen Traditionen seit langem beheimatet ist, in internationalen Menschenrechtsdokumenten bis dato aber nicht vorgekommen war, nämlich im Begriff der individuellen Autonomie. ${ }^{41}$ Dies ist überraschend. Denn generell neigen Juristinnen und Juristen dazu, sich an etablierte Rechtsbegriffe zu halten, was sich schon aus Gründen der Rechtsklarheit und Rechtssicherheit empfiehlt. Beim Begriff der individuellen Autonomie handelt es sich aber - jedenfalls für den Bereich des internationalen Menschenrechtsschutzes - um eine Innovation. Dies birgt nicht nur Chancen, sondern auch gewisse Risiken, weil sich ein internationales "case law«, durch das der Begriff konkretere Konturen erhalten kann, erst noch entwickeln muss.

Offenbar ging es den Verfasserinnen und Verfassern der Konvention, an deren Entstehen Behindertenverbände aktiv beteiligt waren, um eine bewusste Akzentsetzung. Im Hintergrund stehen die Unrechtserfahrungen von Menschen, die oft bis heute in beschützten Werkstätten, Heimen und anderen Betreuungseinrichtungen bevormundet werden. Die Prägnanz des

39 Vgl. Krennerich (2006), 57-66.

40 CESCR E/C.12/2000/4, 11. August 2000, Ziff. 8.

41 Vgl. Artikel 3 Absatz a, Grundsatz a der UN-Konvention über die Rechte von Personen mit Behinderungen. 
Autonomiebegriffs in der Behindertenrechtskonvention entfaltet dagegen eine kritische Signalwirkung. Gefordert wird der Respekt vor freier individueller Selbstbestimmung, der sich auf alle Lebensbereiche bezieht: die Gestaltung des eigenen Wohnumfelds; den Schutz einer unantastbaren Privatsphäre; eigene Entscheidungen in Sachen Partnerschaft, Sexualität, Ehe und Familie; die Bereiche Schule und Bildung; frei gewählte Berufstätigkeit; die aktive Mitwirkung am politischen Leben usw. Alle konkreten Rechtsverbürgungen innerhalb der Behindertenrechtskonvention sind Ausdruck dieses Anspruchs auf Achtung selbstbestimmter Lebensführung, der im Leitbegriff der individuellen Autonomie verdichtet wird. ${ }^{42}$

Zugleich formuliert die Konvention Ansprüche auf ein offenes und unterstützendes soziales Umfeld, durch das Autonomie überhaupt erst realisiert werden kann. Dafür hat die Konvention ebenfalls einen Begriff geprägt, der zuvor im Menschenrechtskontext wenig etabliert war, nämlich den Begriff der Inklusion. ${ }^{43}$ Auch hinter diesem Begriff stehen Unrechtser-

42 In der praktischen Philosophie Kants bezeichnet der Begriff der sittlichen Autonomie die grundlegende Verantwortungssubjektivität des Menschen, die dem Recht vorausliegt und in Gestalt rechtlicher Freiheit letztlich nur indirekt geschützt werden kann. Wenn man so will, stellt die Kantische Autonomie eine philosophische Klärung dessen dar, was zuvor unter der Formel von der Ausstattung des Menschen mit »Vernunft und Gewissen« landläufig angesprochen wird. Es gilt demnach, zwischen Autonomie als einer Kategorie der Moralphilosophie und Selbstbestimmung als einer Kategorie der Rechtsphilosophie klar zu unterscheiden. Vgl. Bielefeldt (2003), 101-107. In der Behindertenrechtskonvention werden die Begriffe Autonomie und Selbstbestimmung demgegenüber anscheinend synonym verwendet. Ich orientiere mich im Folgenden an diesem Sprachgebrauch und verwende Autonomie und Selbstbestimmung demnach hier als austauschbare Begriffe.

43 Vgl. Artikel 3 Absatz c der Konvention für die Rechte von Personen mit Behinderungen. In der offiziellen deutschen Übersetzung kommen die Begriffe »Inklusion« bzw. »inklusiv« erstaunlicherweise gar nicht vor. Gegen den ausdrücklichen Widerspruch der Behindertenverbände wurden sie durch »Integration« bzw. »integrativ« ersetzt. Dabei geht der Anspruch der Inklusion ausdrücklich über Integration hinaus, insofern es nicht nur darum geht, Menschen mit Behinderung in die bestehenden gesellschaftlichen Subsysteme, soweit wie möglich, 
fahrungen von Menschen, die aus vielen Lebensbereichen systematisch ausgegrenzt waren oder es noch sind.

Individuelle Autonomie und soziale Inklusion gehören in der Behindertenrechtskonvention als Leitbegriffe aufs Engste zusammen und beleuchten einander wechselseitig. Aus der Erfahrungsperspektive von Menschen, die oft gleichermaßen unter Bevormundung wie unter gesellschaftlichem Ausschluss leiden mussten, wird hier der doppelte Anspruch formuliert: Gesellschaftliches Dabeisein - und zwar ohne Bevormundung! Persönliche Selbstbestimmung - aber nicht als Vorwand für soziale Isolierung! Der Begriff der Autonomie verliert in dieser Verschränkung mit dem Begriff der Inklusion seinen harten, »metallenen« Klang, der ihm in manchen neo-liberalen Debattenkontexten anhaftet. Autonomie meint gerade nicht die Autarkie des ganz auf sich gestellten Heroen, von dem einst die Stoiker träumten, sondern verlangt, dass Menschen - gerade auch in vulnerablen Lebenslagen - stets als Verantwortungssubjekte behandelt werden und in ihrem Anspruch auf individuelle Selbstbestimmung gesellschaftliche Resonanz und Unterstützung finden. ${ }^{44}$

Die Verschränkung von Autonomie und gesellschaftlicher Unterstützung, wie sie durch die Behindertenrechtskonvention geleistet wird, hat erhebliche Relevanz für die Menschenrechtsdebatte im Ganzen - weit über das Themenfeld Behinderung hinaus. Insbesondere gilt dies für Menschenrechte im Kontext des Gesundheitswesens. In von Krankheit, schwerer Behinderung, Demenz und hohem Alter geprägten Lebenslagen benötigen Menschen unter Umständen weitreichende Unterstützungsleistungen, um überhaupt selbstbestimmt leben - oder auch nur selbstbestimmt entscheiden - zu können. ${ }^{45}$ In Grenzsituationen kann es darüber hinaus sogar erforderlich sein, den Willen eines Betroffenen, der sich nicht mehr direkt äußern kann, auf indirektem Wege zu erschließen. Personale, soziale oder technische Unterstützungsleistungen, die zugunsten der Autonomie eines Menschen eingesetzt werden, sind in jedem Fall zugleich kritisch an der Autonomie des Betroffenen zu bemessen, die durch Förderung nicht gleichsam von außen "geschaffen« wird, sondern immer zugleich als unverfügbar

mit hineinzulassen, sondern Gesellschaft insgesamt unter dem Anspruch »Dabeisein von Anfang an « neu zu denken und zu gestalten.

44 Vgl. Graumann (2009).

45 Vgl. die Beiträge in Aichele (2013). 
vorgegeben zu achten ist. Autonomie ist für die Rechtsgemeinschaft insofern stets Aufgabe und Vorgabe zugleich. Nur in dieser Doppelstruktur kann sie zur Geltung kommen.

Eine große Rolle spielt der Autonomiebegriff bekanntlich in Debatten über aktive Sterbehilfe und assistierten Suizid. Häufig kommt dabei ein Verständnis von Autonomie zum Zuge, das diese als unbeschränkte Selbstverfügung des Menschen fasst. Die Möglichkeit, über das eigene Leben zu verfügen und es aus freiem Entschluss zu beenden, gilt gelegentlich als eigentlicher Testfall freier individueller Selbstbestimmung bzw. Autonomie. ${ }^{46}$ Hier ist Vorsicht geboten. Denn die freie Selbstbestimmung, auf die jeder Mensch ein Recht hat, gründet zuletzt in der Anerkennung der Würde des Menschen als eines mit Vernunft und Gewissen ausgestatteten Verantwortungssubjekts. ${ }^{47}$ Im Begriff der Selbstbestimmung schwingt im Menschenrechtskontext insofern stets die Komponente der Selbstverantwortung mit. Was aber bedeutet dies in der Praxis? Heißt es, dass die Selbstbestimmung dort ihre Grenzen findet, wo der Menschen von seiner Freiheit einen »unverantwortlichen« Gebrauch macht? Wer aber könnte darüber ggf. entscheiden?

Relativ trivial ist zunächst die Feststellung, dass die Freiheit ihre Grenze an der Freiheit der Anderen findet. Wo genau die Grenze gezogen wird, mag im Einzelnen zwar komplizierte Fragen aufwerfen; im Prinzip ist aber unbestritten, dass es solche sozialen Grenzen geben muss. Denn das Recht auf freie Selbstbestimmung kann kein Freibrief zur Zerstörung der Freiheit Anderer sein. Ausgesprochen kontrovers diskutiert wird demgegenüber die Frage, ob es auch »immanente« Grenzen freier Selbstbestimmung gibt, also Grenzen, die in Pflichten des Menschen gegenüber sich selbst gründen. Schließt die Freiheit die Möglichkeit ein, sich selbst zu verstümmeln, Experimente mit hohem Risiko an sich selbst durchzuführen oder auch seinem Leben in freiem Entschluss ein Ende zu setzen?

Gewiss ist die Freiheit nicht zu haben ohne die Risiken der Freiheit. Menschen haben die Freiheit, sich ins Unglück zu stürzen, ihr Geld falsch anzulegen, vorschnell eine Ehe einzugehen und einen Lebensstil zu pflegen, der unter Gesichtspunkten der Gesundheitspflege fragwürdig sein

46 So der Tenor der meisten Beiträge in Hegselmann/Merkel (1991).

47 Die Behindertenrechtskonvention führt die Begriffe der individuellen Autonomie und der inhärenten Menschenwürde gemeinsam in Artikel 3 Absatz a auf. 
kann. Dennoch gibt es gute Gründe, der individuellen Freiheit zuletzt auch immanente (und nicht nur soziale) Grenzen einzuziehen. Diese sind spätestens dort erreicht, wo der Mensch sich aus freien Stücken als Subjekt von Freiheit selbst unwiderruflich aufgibt. In den Debatten des 18. Jahrhunderts wurde dies vor allem am Beispiel der Selbstversklavung diskutiert. Kann der Mensch sich aus eigenem Entschluss unwiderruflich selbst verkaufen? Erst in der klaren Absage an diese (noch im Zeitalter der Aufklärung durchaus verbreitete) Vorstellung entstand das Konzept »unveräußerlicher« Freiheitsrechte. ${ }^{48}$ Freiheit meint dabei gerade nicht die Möglichkeit der individuellen Selbstverfügung ohne Wenn und Aber, sondern die Achtung vor der umfassenden Verantwortungssubjektivität eines jeden Menschen nicht nur bei anderen, sondern auch bei sich selbst. Ein Ja zu einem Recht auf Selbsttötung im Namen der Autonomie erweist sich in dieser Perspektive als problematisch. Wie Volker Gerhardt in einer Diskussion des Nationalen Ethikrats zur Sterbehilfe betonte: »Es gehört zur Logik dieser Selbstbestimmung, dass sie sich tatsächlich auch als solche erhält. « ${ }^{49}$

Im Horizont des Menschenrechtsansatzes, der systematisch bei der Würde des Menschen als Verantwortungssubjekt ansetzt, gründet Selbstbestimmung letztlich in Selbstverantwortung und eben nicht in grenzenloser Selbstverfügung. Andernfalls ergäbe der Begriff der »unveräußerlichen« Menschenrechte keinen Sinn. So verstanden ist Selbstbestimmung aber nicht nur ein Anspruch des Menschen, sondern eben auch ein Anspruch an den Menschen. Sie ist ein - auch moralisch - »anspruchsvolles« Konzept.

Bei der Durchsetzung dieses moralisch anspruchsvollen Konzepts mittels Rechtszwang stößt die Rechtsordnung allerdings an ihre Grenzen. Aus guten Gründen soll der Rechtsstaat kein Tugendstaat sein, der nach allen Erfahrungen stets nur auf eine Karikatur staatlich verordneter Moral hinausläuft. Eine moralische oder gar rechtliche "Verdammung « von Suizidenten erscheint daher als unangemessen. Der Versuch der Selbsttötung wird heute, anders als in der Vergangenheit, nicht mehr strafrechtlich sanktioniert. Auch Menschen, die bei der Selbsttötung Unterstützung leisten, bleiben in der Regel straffrei. Darüber hinaus liegt es der modernen Gesellschaft inzwischen fern, Selbsttötung moralisierend als Selbst-»Mord « zu stigmatisieren. Dahinter steht die Einsicht, dass es in den meisten Fällen

48 Vgl. Willoweit (1992), 255-268.

49 Gerhardt (2004), 9. 
nicht möglich ist, die inneren Motive eines Menschen, der seinem Leben ein Ende bereitet, angemessen zu erfassen, geschweige denn zu bewerten. Auch die Kirchen weigern sich längst nicht mehr, Suizidenten wie alle anderen Menschen zu beerdigen.

Diese Zurückhaltung - oft sogar Scheu - in der konkreten Bewertung einer Selbsttötung ist aber nicht zu verwechseln mit einer positiven Anerkennung eines Rechtsanspruchs in diesem Feld. Ein »Menschenrecht auf Selbsttötung « gibt es genauso wenig wie ein Recht auf professionelle Assistenz bei der Selbsttötung. ${ }^{50}$ Meiner Überzeugung nach muss es dabei bleiben. Gerade im Ernstnehmen der Selbstbestimmung wird klar, dass das Subjekt der Selbstbestimmung sich nicht aus freien Stücken unwiderruflich selbst aufgeben kann. Die Selbstaufgabe als konkreter Akt der Verzweiflung entzieht sich zwar jedem moralisierenden Kommentar, dies ist klar. Den Lobbyisten des professionell assistierten Freitods ist aber zu widersprechen - und zwar gerade im Namen einer anspruchsvoll verstandenen Autonomie. $^{51}$

Der Begriff der Autonomie spielt nicht nur in Diskussionen über das Lebensende eine Rolle. Jürgen Habermas hat ihn in seiner Kritik an Projekten einer »liberalen Eugenik« auch auf den Lebensanfang, nämlich den gesellschaftlichen Umgang mit vorgeburtlichem menschlichem Leben, bezogen. $^{52}$ Dies geschieht nicht etwa in der Weise, dass Habermas bereits Embryonen oder Föten eine rudimentäre Autonomie zuerkennen würde. Den Ausgangspunkt seiner Überlegungen bilden vielmehr die bereits gebo-

50 Vgl. das Urteil des Europäischen Gerichtshofs für Menschenrechte vom 29. April 2002 im Fall Diane Pretty gegen das Vereinte Königreich. Die Forderung der gelähmten Frau, sich von ihrem Ehemann ein tödliches Mittel verabreichen zu lassen, um so den befürchteten Erstickungstod zu vermeiden, wurde vom Gerichtshof zurückgewiesen, der dem Staat die Möglichkeit einräumte, entsprechende Verbotsregelungen zur Verhinderung sozialethisch problematischer Folgewirkungen zu erlassen. Am Wortlaut der Urteilsbegründung merkt man, wie schwer sich die Richterinnen und Richter mit dieser Entscheidung getan haben. Angemerkt sei, dass angesichts der heutigen Möglichkeiten der Palliativmedizin die Angst vor einem quälenden Erstickungstod stark relativiert werden muss.

51 Vgl. in diesem Sinne auch die im November 2015 verabschiedete Novellierung von $\S 217$ StGB.

52 Vgl. Habermas (2005). 
renen Menschen und ihre soziale Interaktion. In seinem sehr nachdenklichen Essay zur »Zukunft der menschlichen Natur« wirft Habermas die Frage auf, ob nicht das für die moderne Demokratie konstitutive Autonomiebewusstsein der Menschen auf Dauer beeinträchtigt wird, wenn sich Praktiken einseitiger genetischer Programmierung immer mehr durchsetzen, die - anders als Einflüsse von Erziehung und Sozialisation - nicht kommunikativ »aufgearbeitet« werden können, sondern als harte biologische bzw. biotechnische Fakten daherkommen. ${ }^{53}$ Er bestreitet dabei nicht, dass ein geklonter Mensch, wenn es ihn denn dereinst geben sollte, wie jeder andere Mensch zur selbstbestimmten Lebensführung in der Lage sein kann und er in jedem Fall in seiner Autonomie respektiert werden muss. Habermas fragt stattdessen nach den langfristigen Folgen, wenn bis dato eher implizite »gattungsethische« Prämissen brüchig werden, an denen unser gesellschaftliches Autonomieverständnis hängt - etwa die Prämisse, dass Menschen ihren je eigenen biographischen Anfang haben und nicht einfach technisch »gemacht« werden. In der allmählichen Erosion solcher Prämissen sieht Habermas langfristige Gefahren für die demokratische Interaktion freier und gleicher Bürgerinnen und Bürger. ${ }^{54}$ Aus dieser Sorge heraus fordert er einen behutsamen Umgang mit vorgeburtlichem menschlichem Leben, das nicht zum Objekt beliebigen Hantierens, Experimentierens, Planens und Optimierens geraten dürfe. Zwar falle es nicht unter die grundrechtlich geschützte »Unantastbarkeit« der Menschenwürde, die den geborenen Menschen vorbehalten sei; gleichwohl komme auch dem vorgeburtlichen Leben eine spezifische »Unverfügbarkeit« zu, aus der sich Grenzen für Embryonenforschung, Präimplantationsdiagnostik und andere biotechnische Projekte ergeben. ${ }^{55}$ Mit seinem Essay hat Habermas viel Zu-

53 Vgl. Habermas (2005), 105-125.

54 Vgl. ebd., 115: „Wie wir mit menschlichem Leben vor der Geburt (oder mit Menschen nach ihrem Tod) umgehen, berührt unser Selbstverständnis als Gattungswesen. Und mit diesem gattungsethischen Selbstverständnis sind die Vorstellungen von uns als moralischen Personen eng verwoben. Unsere Auffassungen von - und unser Umgang mit - vorpersonalem menschlichen Leben bilden sozusagen eine stabilisierende gattungsethische Umgebung für die vernünftige Moral der Menschenrechtssubjekte - einen Einbettungskontext, der nicht wegbrechen darf, wenn nicht die Moral selbst ins Rutschen kommen soll.«

55 Vgl. Habermas (2005), 149. 
stimmung, aber auch scharfen Widerspruch hervorgerufen. In jedem Fall ist es ihm zu verdanken, dass das Thema Autonomie - vermittelt über gattungsethische Voraussetzungen des gesellschaftlichen Autonomiebewusstseins - mittlerweile auch bezüglich des Lebensanfangs debattiert wird.

Erstaunlich selten werden die Begriffe Autonomie und Selbstbestimmung in der öffentlichen Debatte auf die im Gesundheitsbereich tätigen Personen - Ärztinnen und Ärzte, Krankenschwestern und Pfleger - bezogen. Dabei gilt natürlich auch für sie, dass ihr Status als Verantwortungssubjekte keineswegs in den professionell umschriebenen und in Arbeitsverträgen ausgestalteten Rollen aufgeht, sondern diesen vorausliegt. Daraus können schwierige Konflikte im Beruf entstehen. So weigern sich beispielsweise manche Ärztinnen und Ärzte aus religiösen oder anderen Überzeugungen, bestimmte medizinische Handlungen vorzunehmen, auch wenn dies Bestandteil ihrer professionellen Funktion ist. Ein klassisches Beispiel ist die Verweigerung der Mitwirkung an Abtreibungen aus Gewissensgründen. Die Rechtsordnungen der Staaten - auch der europäischen Staaten gehen mit solchen Konflikten sehr unterschiedlich um. ${ }^{56}$

Die Verweigerung bestimmter medizinischer Handlungen aus Gewissensgründen fällt prinzipiell in den Anwendungsbereich der Gedanken-, Gewissens- und Religions- und Weltanschauungsfreiheit, hat also eine menschenrechtliche Dimension. ${ }^{57}$ Gleichwohl kommt dieses Thema in der internationalen Menschenrechtsdiskussion erstaunlicherweise nur selten vor. Vielleicht steht dahinter die Sorge, dass eine formelle Anerkennung eines Rechts auf gewissensbedingte Weigerung in diesem weiten Feld zu Beeinträchtigungen der Funktionsfähigkeit des Gesundheitswesens - und damit evtl. zu anderen menschenrechtlichen Problemen - führen könnte. ${ }^{58}$ Ob diese Gefahr tatsächlich besteht und wie ggf. damit angemessen - d.h. im Bemühen um möglichst schonenden Ausgleich konkurrierender Ansprüche - umzugehen wäre, bedarf freilich einer genauen Analyse. Es wäre

56 Vgl. E.U. Network of Independent Experts on Fundamental Rights, Opion no. 42005L: »The Right to Conscientious Objection and the Conclusion by EU Member States of Concordats with the Holy See« (14 December 2005), Ref. CRF-CDR.Opinion 4-2005.doc, 20.

57 Vgl. Bielefeldt (2013), 33-68.

58 Dies betrifft etwa die Gewährleistung der sexuellen und reproduktiven Rechte für alle. 
jedenfalls nicht legitim, den im Gesundheitsbereich Tätigen ihr Recht, den eigenen Gewissensüberzeugungen entsprechend zu handeln oder ggf. auch die Vornahme bestimmter Handlungen zu verweigern, mit Blick auf unspezifische Überlegungen zur Funktionsfähigkeit des Gesundheitssystems pauschal abzusprechen. Hier scheint mir erheblicher Klärungs- und Diskussionsbedarf zu bestehen.

\section{GLEICHHEIT UND NICHT-DISKRIMINIERUNG}

Wie die Freiheit ist auch die Gleichheit eines der architektonischen Prinzipien, auf denen der Menschenrechtsansatz im Ganzen fußt. Die in der Würde des Menschen gegründeten fundamentalen Freiheitsrechte müssen einem jeden Menschen gleichermaßen zukommen - sonst wären sie keine universalen Menschenrechte. Schon die Präambel der Allgemeinen Erklärung formuliert einen direkten Zusammenhang zwischen »der allen Mitgliedern der menschlichen Familie inhärenten Würde« und ihren »gleichen und unveräußerlichen Rechten«. Und Artikel 1 der Allgemeinen Erklärung beginnt mit dem bereits zitierten berühmten Postulat, dass »alle Menschen frei und an Würde und Rechten gleich geboren« sind.

Freiheit und Gleichheit sind als Menschenrechtsprinzipien eng aufeinander bezogen und erhellen einander wechselseitig. Mit Blick auf das Gleichheitsprinzip bleibt deutlich, dass die Freiheitsrechte nicht bloße Privilegien einiger Begünstigter sein können; und mit Blick auf das Freiheitsprinzip wird klar, dass die menschenrechtliche Gleichheit nicht auf Gleichförmigkeit, Uniformierung oder Nivellierung hinauslaufen kann - ganz im Gegenteil. ${ }^{59}$ Um es auf Englisch zu sagen: »equality« meint nicht »sameness «. Ziel ist es, dass die Menschen gerade in ihren vielfältigen Besonderheiten Berücksichtigung finden - aber eben so, dass dies kein Privileg einiger Weniger bleibt, sondern die bestehenden Möglichkeiten allen gleichermaßen zukommen. Die Gleichheit, um die es in den Menschenrechten geht, ist von vornherein eine komplexe Gleichheit, die auf unterschiedliche Biographien, Prägungen, Überzeugungen, Vulnerabilitäten und Nöte Rücksicht nimmt. Gendersensibilität, Behindertensensibilität oder Kultursensibilität

59 »All different, all equal« lautet der gleichermaßen knappe wie treffende Slogan einer Kampagne des Europarats. 
stehen mit der so verstandenen Gleichheit nicht nur keineswegs in Widerspruch, sondern sind ihr gleichsam eingeschrieben. ${ }^{60}$

Seine konkreten rechtlichen Konturen erfährt das Gleichheitsprinzip vor allem durch das Diskriminierungsverbot, das sich in allen einschlägigen Menschenrechtsdokumenten findet. Laut Artikel 2 der Allgemeinen Erklärung der Menschenrechte hat jeder Mensch Anspruch auf alle in der Erklärung aufgeführten Rechte,

»ohne Unterscheidung irgendwelcher Art, wie etwa aufgrund von Rasse, Hautfarbe, Geschlecht, Sprache, Religion, politischer oder sonstiger Überzeugung, nationaler oder sozialer Herkunft, Besitz, Geburt oder sonstigem Status«.

Die Formulierung (im Englischen prägnanter: »without distinction of any kind «) macht deutlich, dass die hier explizit genannten Anknüpfungspunkte exemplarisch zu verstehen sind und insofern für Erweiterungen offen bleiben.

Tatsächlich hat es, durch soziale Bewegungen und transnationale NGOs angestoßen, in den letzten Jahrzehnten erhebliche Ausweitungen innerhalb des Diskriminierungsverbots gegeben. Jüngere Menschenrechtsdokumente führen Diskriminierungsmerkmale auf, an die 1948 noch kaum jemand gedacht hatte. Dies gilt etwa für das Merkmal Behinderung. ${ }^{61}$ Aus dem Diskriminierungsverbot leitet die Behindertenrechtskonvention nicht nur das allgemeine Ziel einer barrierefreien Gesellschaft $a b ;{ }^{62}$ sie verleiht Menschen mit Behinderung darüber hinaus einen Rechtstitel auf Durchführung konkreter Umweltanpassungen, um ihnen die Inklusion in gesellschaftliche Institutionen zu ermöglichen. ${ }^{63}$ Obwohl in einigen Regionen der Welt noch

60 Vgl. CESCR E/C.12/2000/4, 11. August 2000, Ziff. 20-27.

61 Dies ist erstaunlich, wenn man bedenkt, dass die internationale Botschafterin der Allgemeinen Erklärung der Menschenrechte, Eleanor Roosevelt, Witwe eines Mannes war, der die letzten Jahre seines Lebens als amerikanischer Präsident weitgehend im Rollstuhl zugebracht hatte.

62 Vgl. Artikel 9 der Konvention für die Rechte von Personen mit Behinderungen.

63 Dieser Anspruch findet sich in Artikel 5 Absatz 3 der Konvention für die Rechte von Personen mit Behinderungen unter dem Begriff »reasonable accommodation«. Artikel 2 enthält dafür folgende Definition: »)Reasonable accommodation< means necessary and appropriate modification and adjustments not imposing a 
stark umstritten, hat sich auch das Verbot der Diskriminierung aufgrund von sexueller Orientierung bzw. Gender-Identität in jüngerer Zeit immer mehr durchgesetzt. ${ }^{64}$ Die damalige UN-Hochkommissarin für Menschenrechte, Navi Pillay, setzte ein Zeichen, als sie im Dezember 2011 einen ausführlichen Bericht zur Diskriminierung sexueller Minderheiten im UNMenschenrechtsrat vorlegte. ${ }^{65}$ Ein vergleichsweise neues Arbeitsfeld stellt die Diskriminierung aufgrund des Alters dar - was übrigens nicht nur ein Thema für Senioren ist, sondern Menschen jedweden Alters betreffen kann, sofern sie aufgrund ihres jeweiligen Lebensalters Diskriminierungen erfahren. Derzeit wird darüber beraten, ob eine eigene internationale Konvention zu altersbezogener Diskriminierung entwickelt werden soll. Die im Dezember 2009 in Kraft getretene EU-Grundrechtecharta enthält darüber hinaus auch ein Verbot von Diskriminierung aufgrund bestimmter genetischer Merkmale eines Menschen. ${ }^{66}$ Die damit verbundenen Rechtsfragen - etwa im Versicherungswesen - sind noch lange nicht systematisch erschlossen worden. Für all die gerade ausgeführten, erst in jüngerer Zeit anerkannten Diskriminierungsmerkmale gilt, dass ihre Relevanz für das Gesundheitswesen auf der Hand liegt. ${ }^{67}$

Antidiskriminierungspolitik verlangt mehr als die Überwindung direkter und offenkundiger Diskriminierung - etwa von Apartheid-Strukturen im Gesundheitswesen, die angeblich einst dazu führten, dass die afro-amerikanische Blues-Sängerin Bessy Smith nach einem Autounfall verblutete. Es geht heute darüber hinaus auch darum, versteckte Formen von Diskriminierung auszuräumen, darunter indirekte und strukturelle Diskriminierungen, die nicht immer leicht zu erkennen sind und deren rechtliche Bewertung nicht immer eindeutig ausfällt. So könnte es sein, dass zwingende

disproportionate or undue burden, where needed in a particular case, to ensure to persons with disabilities the enjoyment or exercise on an equal basis with others of all human rights and fundamental freedoms."

64 Vgl. Baer (2009), 89-118.

65 Vgl. Discriminatory laws and practices and acts of violence against individuals based on their sexual orientation and gender identity. Report of the United Nations High Commissioner for Human Rights, Navenethem Pillay, UN Doc. A/HRC/19/41.

66 Vgl. Artikel 21 Absatz 1 der EU-Grundrechtscharta.

67 Vgl. auch CESCR E/C.12/2000/4, 11. August 2000, Ziff. 18. 
Regelungen zum Schichtbetrieb in einem Krankenhaus für alleinerziehende Frauen auf einen faktisch-diskriminierenden Ausschluss aus entsprechender Berufstätigkeit hinauslaufen. Wenn die Krankenhauskost keine Rücksicht auf die Speisevorschriften von Juden, Muslimen oder anderen religiösen Minderheiten nimmt, entstehen hohe Hürden für die Angehörigen der entsprechenden Religionsgemeinschaften. ${ }^{68}$ Kostenbeteiligungen können den Zugang für ärmere Bevölkerungsgruppen zur Gesundheitsversorgung erheblich erschweren. Manche Menschen haben aufgrund ihrer Lebensumstände Schwierigkeiten, zu annehmbaren Konditionen in eine Krankenversicherung aufgenommen zu werden. Solche Fragen stehen heute unter der Problemanzeige indirekter bzw. struktureller Diskriminierung. Dabei geht es nicht primär darum, den betreffenden Institutionen das Stigma der Diskriminierung anzuhängen, sondern positiv um Öffnung der Institutionen. Denn der menschenrechtliche Charakter des Rechts auf Gesundheit und anderer Rechte im Gesundheitswesen hängt in der Praxis daran, dass sie ohne jedwede Diskriminierung - auch ohne versteckte Formen von Diskriminierung - in Anspruch genommen werden können.

\section{Die GaRAntenfunktion des StaAtes}

Es ist Zeit, einen Aspekt, der in den bisherigen Ausführungen eher implizit präsent war, nun auch explizit anzusprechen, weil ohne ihn die Menschenrechte nicht verstanden werden können: nämlich deren positiv-rechtliche Gestalt. $^{69}$ Gewiss lassen sich Menschenrechte zunächst als moralische »Werte« verstehen, sind sie doch Ausdruck eines moralischen Universalismus, der jedem Menschen um seiner Würde als Verantwortungssubjekt willen gleiche Freiheit zuspricht. ${ }^{70}$ Menschenrechte zielen darüber hinaus aber auch auf wirksame Rechtsdurchsetzung. Dazu braucht es eine komplexe Infrastruktur von nationalen und übernationalen Gerichten, Ombudsinstitutionen, Monitoring-Verfahren, anlassunabhängigen Überprüfungen, peri-

68 Solche Fragen werden seit längerem unter »reasonable accommodation « diskutiert und teils auch vor Gerichten entschieden. Vgl. dazu aus spezifisch USamerikanischer Perspektive Nussbaum (2008), 115-174.

69 Vgl. Sandkühler (2007), 57-86.

70 Vgl. Habermas (2010), 43-53. 
odischer Berichterstattung usw. Menschenrechte fungieren als Movens für den Aufbau und Ausbau komplexer Rechtsinstitutionen auf nationaler, regionaler und globaler Ebene, die sich wechselseitig ergänzen sollen. Diese positiv-rechtliche Seite der Menschenrechte ist keine bloße Äußerlichkeit, sondern gehört zu ihrem Proprium. Sie prägt auch den Wortlaut menschenrechtlicher Ansprüche, die von vornherein so gestaltet sind, dass sie tatsächlich im Medium des Rechts durchgesetzt werden können. Als Durchsetzungsinstanz fungiert dabei der Staat, der sich in nationalen Verfassungen bzw. durch Ratifizierung regionaler und globaler Menschenrechtskonventionen förmlich zur Garantie der Menschenrechte verpflichtet. Menschenrechte richten sich deshalb in ihrer politisch-rechtlichen Gestalt primär (wenn auch nicht exklusiv) an den Staat. Ihm obliegt es, Menschenrechte zu achten - woraus sich Schranken legitimer Staatstätigkeit ergeben - und sie zugleich durch Schutz- und Infrastrukturmaßnahmen aktiv zur Geltung zu bringen. ${ }^{71}$

Auch für den Staat gilt dabei natürlich, dass man ihm nichts Unmögliches abverlangen kann. »Ultra posse nemo obligatur« wussten schon die römischen Rechtsgelehrten. Gerade das Recht auf Gesundheit führt hier immer wieder zu skeptischen Rückfragen. Sind die Möglichkeiten des Staates in diesem Feld nicht allzu sehr abhängig vom ökonomischen und technischen Entwicklungsstand einer Gesellschaft - und nicht zuletzt auch von der wechselnden Budget-Lage? Kommen damit nicht zwangsläufig allerlei sozio-ökonomische, technische, finanzpolitische und andere Vorbehalte mit ins Spiel? Heißt das aber nicht, dass Rechtsansprüche in diesem Feld fernab von der für Menschenrechte üblichen Unbedingtheits-Semantik - in starke Abhängigkeit von kontingenten Faktoren geraten? Muss dies nicht auf Dauer zu einer Schwächung der Menschenrechte führen, die sich auf solch schwankenden Boden begeben? Und schließlich ganz schlicht: Lässt sich Gesundheit der Natur der Sache nach überhaupt menschenrechtlich normieren, einklagen und durchsetzen?

71 Dies bedeutet, dass dem Staat unterschiedliche Pflichten zukommen, die neuerdings gern als Pflichtentrias - nämlich als Achtungs-, Schutz- und Gewährleistungspflichten - ausdifferenziert werden. Vgl. ähnlich auch CESCR E/C.12/ 2000/4, 11. August 2000, Ziff. 33 und den Beitrag von Krennerich in diesem Band. 
Wichtige systematische Klärungen, die der UN-Ausschuss für wirtschaftliche, soziale und kulturelle Rechte (WSK-Ausschuss) sowie die einschlägigen UN-Sonderberichterstatter in den letzten Jahren erarbeitet haben, machen es möglich, auf solche skeptischen Fragen zu antworten. ${ }^{72}$ Zunächst stellt der General Comment des WSK-Ausschusses klar, dass das Recht auf Gesundheit natürlich nicht mit einem Recht auf »Gesundsein« verwechselt werden darf. ${ }^{73}$ Ein solches Recht kann es nicht geben. Menschenrechte sind keine Erlösungslehre, mit Hilfe derer man Leid und Krankheit aus der Welt schaffen könnte. Vielmehr geht es zentral darum, jedem Menschen einen diskriminierungsfreien Zugang zu einem - sich gesellschaftlich stets entwickelnden - Gesundheitssystem rechtlich zu gewährleisten. Die damit verbürgten materialen Leistungen differieren nach Maßgabe des Entwicklungsstandes einer Gesellschaft. Sie sehen in Schweden sicherlich ganz anders aus als in Sierra Leone und liegen in Abu Dhabi wiederum völlig anders als in Kambodscha. Hinsichtlich des formalen Anspruchs auf diskriminierungsfreien Zugang schlagen technische und sozioökonomische Entwicklungsdifferenzen aber weit weniger zu Buche. Dass niemand aufgrund von Geschlecht, Alter, Behinderung oder eines anderen Merkmals ausgegrenzt werden darf, gehört zu den unabdingbaren Forderungen, denen alle Gesellschaften gleichermaßen unterliegen, hochentwickelte Länder genauso wie Entwicklungsländer.

Der vollständige Titel des Rechts, das meist als »Recht auf Gesundheit« abgekürzt wird, lautet: »das Recht eines jeden auf das für ihn erreichbare Höchstmaß an körperlicher und geistiger Gesundheit «. ${ }^{74}$ Genau besehen, enthält diese Formulierung unterschiedliche Ansprüche, die auch hinsichtlich ihres juristischen Verpflichtungsmodus und ihres juristischen Durchsetzungsmodus stets klar differenziert werden müssen. ${ }^{75}$ Auf der einen Seite stehen Staaten in der Pflicht, das gesellschaftliche Potenzial in Sachen

72 Vgl. dazu grundsätzlich Riedel (2008), 71-93.

73 Vgl. CESCR E/C.12/2000/4, 11. August 2000, Ziff. 8.

74 So Artikel 12 des Internationalen Pakts über wirtschaftliche, soziale und kulturelle Rechte.

75 Die folgenden Ausführungen erheben nicht den Anspruch, die verschiedenen Gewährleistungsebenen des Rechts auf Gesundheit vollständig darzulegen. Es geht mir lediglich darum, auf die immanente Mehrdimensionalität des Rechts als solche hinzuweisen. 
Gesundheitswesen sukzessive zu entwickeln und dafür konkrete Pläne vorzulegen. Gesundheitspflege ist keine statische Kategorie. Die Möglichkeiten, die sich hier auftun, sollen genutzt werden, und den Staaten obliegt es, hierfür einen geeigneten Rahmen zur Verfügung zu stellen. Dies schließt transparente Planungen (mitsamt klar definierten Etappenzielen), angemessene Forschungsförderung, qualifizierte Ausbildung medizinischen Fachpersonals, breit angelegte öffentliche Gesundheitsbildung und andere Infrastrukturmaßnahmen ein. Auf der anderen Seite sind die Staaten gehalten dafür zu sorgen, dass die Menschen die jeweils mögliche Gesundheitspflege bereits hier und jetzt wirksam in Anspruch nehmen können, und zwar ohne Ausschluss und ohne Diskriminierung. Das Recht auf Gesundheit ist keine utopische Kategorie, sondern muss auch unter den jeweils aktuellen Verhältnissen bereits wirksam werden. Es gilt hic et nunc. ${ }^{76}$

Beim Menschenrecht auf Gesundheit handelt es sich somit um ein komplexes Recht. ${ }^{77}$ Genau besehen, kombiniert es unterschiedliche Dichtegrade positiv-rechtlicher Verpflichtung des Staates und unterschiedliche Modi rechtlicher Durchsetzung. ${ }^{78}$ Während Staaten hinsichtlich der gebotenen diskriminierungsfreien Gewährleistung des Rechts auf Gesundheit wenig oder gar keinen legitimen Ermessensspielraum haben, können sie der Verpflichtung zur Weiterentwicklung des Gesundheitssystems auf unterschiedliche Weise nachgehen; hier ist ihr Ermessensspielraum vergleichsweise groß. Und während bestimmte Kerngehalte des Rechts auf Gesundheit justiziabel sind, also von den betroffenen Menschen eingeklagt werden könnten, ${ }^{79}$ unterliegen andere Aspekte lediglich einem allgemeinen Monitoring nach Gesichtspunkten von Transparenz, angemessener Prioritätenset-

76 Vgl. CESCR E/C.12/2000/4, 11. August 2000, Ziff. 30: »Obgleich der Pakt eine schrittweise Verwirklichung vorsieht und die Zwänge anerkennt, die auf begrenzt verfügbare Ressourcen zurückzuführen sind, erlegt er den Staaten auch verschiedene Verpflichtungen auf, die eine sofortige Wirkung haben.«

77 Vgl. Hunt/Backman (2008), 81-92.

78 Grundsätzlich zu dieser Struktur Riedel (1986).

79 Der Konjunktiv in diesem Satz ist bewusst gewählt. Mit »Justiziabilität« ist zunächst die prinzipielle Eignung bestimmter Rechtsansprüche zur Durchsetzung auf dem Wege individueller Klage gemeint. Ob solche Klagewege in einem Staat tatsächlich vorliegen und wirksam sind, ist eine andere Frage. Vgl. Jakob (2004) sowie auch den Beitrag von Müller im vorliegenden Band. 
zung and »accountability«. Die unterschiedlichen Ebenen des Rechts auf Gesundheit gehören sachlich zusammen und müssen gleichwohl modal differenziert bleiben. Sonst drohen die komplementären Gefahren einer utopischen Überspannung, durch die der Rechtsanspruch seine Anwendbarkeit einbüßen würde, oder einer bloßen Festschreibung des gesundheitspolitischen Status quo, wodurch die Entwicklungsdynamik des Rechts auf Gesundheit verloren ginge.

Wie eingangs dargestellt, kommen die Menschenrechte den Menschen allein aufgrund ihres Menschseins zu; genau darin besteht der menschenrechtliche Universalismus. Die Garantenfunktion des Staates für die Menschenrechte bleibt deshalb weder auf die eigenen Staatsangehörigen beschränkt noch macht sie an den eigenen Staatsgrenzen halt. Die Staatsgrenzen und damit verbundene spezifische Zuständigkeiten der Staaten lösen sich unter dem Anspruch des Universalismus zwar nicht in globaler Allzuständigkeit aller Staaten auf, ${ }^{80}$ sie verlieren aber jedenfalls ihren ehemals hermetischen, exklusiven Charakter. Dies zeigt sich beispielsweise im Umgang mit grenzüberschreitenden freiwilligen oder unfreiwilligen Migrationsbewegungen. Zur freiwilligen Migration zählt die gezielte Inanspruchnahme hochwertiger oder besonders günstiger Gesundheitsleistungen eines anderen Staates durch Menschen, die sich internationale Reisen und Aufenthalte leisten können. ${ }^{81}$ Damit stellen sich Fragen etwaiger Konkurrenz mit anderen Anspruchsberechtigten - besonders dramatisch etwa im Kontext der Transplantationsmedizin. Zu den unfreiwilligen Migrationsbewegungen gehören Flucht und Asyl, aber auch die ganz anders gelagerten Phänomene des Menschenhandels. Die bereits kurz angesprochene Gesundheitsversorgung von Migrantinnen und Migranten ohne Papiere stellt keineswegs das einzige schwierige Thema in diesem Zusammenhang dar. Solche Probleme lassen sich offenkundig nur in der Kooperation der Staaten bzw. im Auf- und Ausbau supranationaler Rechtsstrukturen erfolgversprechend bearbeiten. Diesbezüglich befinden wir uns noch in einem frühen Stadium rechtlicher Entwicklung.

Was außerhalb seiner Grenzen vor sich geht, kann einem Staat, der sich als Garant von Menschenrechten versteht, nicht gleichgültig sein - so weit,

80 Hier zeigt sich einmal mehr die Notwendigkeit, zwischen normativem Universalismus und territorialer Globalität kategorial zu unterscheiden.

81 Vgl. dazu Krajewski (2010), 165-188. 
so klar. Es stellt sich aber die Frage, ob und inwieweit die Verbindlichkeiten, die sich daraus für den Staat ergeben, "nur« moralischer oder politischer Natur sind oder auch genuin juristischen Charakter haben. Diese Frage wird seit einigen Jahren unter dem Begriff der extraterritorialen Staatenpflichten diskutiert. Dass es solche extraterritorialen Staatenpflichten juristischer Natur gibt, ist mittlerweile völkerrechtlich anerkannt. Auch der General Comment des WSK-Ausschusses zum Recht auf Gesundheit enthält diesbezüglich wichtige Hinweise. Der Ausschuss betont zunächst die »negative« extraterritoriale Verpflichtung des Staates, in jedem Fall von solchen Maßnahmen Abstand zu nehmen, die sich auf die Gesundheitssysteme anderer Länder schädlich auswirken könnten. Der Staat ist darüber hinaus verpflichtet, das ihm Mögliche dafür zu leisten, die Verhinderung schädlicher Maßnahmen auch gegenüber Dritten - etwa transnationalen Konzernen - wirksam durchzusetzen. ${ }^{82}$ Wirtschaftsembargos dürfen die Versorgung der Bevölkerung mit wichtigen medizinischen Gütern nicht beeinträchtigen. ${ }^{83}$ Darüber hinausgehende positive Staatenpflichten im extraterritorialen Bereich stehen hingegen unter Ressourcenvorbehalt. ${ }^{84}$ Die Abschichtung unterschiedlicher Dichtegrade rechtlicher Verpflichtung zeigt sich also auch im internationalen Bereich. Auf diese Weise soll der genuin juristische Charakter des Rechts auf Gesundheit in seinen Konturen gewahrt bleiben, ohne die weiter reichenden moralischen oder politischen Verpflichtungen, die sich daraus ergeben, abzuschneiden.

82 Vgl. CESCR E/C.12/2000/4, 11. August 2000, Ziff. 39: »Um ihren internationalen Verpflichtungen aus Artikel 12 nachzukommen, müssen die Vertragsstaaten das Recht auf Gesundheit in anderen Ländern achten und Dritte daran hindern, dieses Recht in anderen Ländern zu verletzen, falls es möglich ist, diese mit rechtlichen oder politischen Mitteln, in Übereinstimmung mit der Charta der Vereinten Nationen und einschlägigem Völkerrecht, zu beeinflussen.«

83 Ebd., Ziff. 41.

84 Ebd., Ziff. 29: »Je nach Verfügbarkeit von Ressourcen sollten die Staaten den Zugang zu elementaren medizinischen Einrichtungen und ärztlicher Betreuung in anderen Ländern, wenn immer möglich, erleichtern und auf Anfrage notwendige Hilfe zur Verfügung stellen.« 


\section{SCHLUSSBEMERKUNGEN}

Ausgangspunkt für die im vorliegenden Text angestellten Grundsatzüberlegungen war die Annahme, dass Menschenrechte im Gesundheitswesen einer breiteren Öffentlichkeit noch wenig bekannt sind. Dass es überhaupt Menschenrechtsansprüche in diesem Gebiet geben kann, scheint für viele Menschen ein neuer, womöglich gewöhnungsbedürftiger Gedanke zu sein. Darüber hinaus stellen sich konzeptionelle wie rechtspraktische Fragen, zu deren Klärung - trotz bahnbrechender Vorarbeiten insbesondere des WSKAusschusses und der einschlägig mandatierten UN-Sonderberichterstatter noch viel Arbeit zu leisten sein wird. So gilt es, den im unbedingten Respekt vor der Würde jedes Menschen als Verantwortungssubjekt gründenden normativen Universalismus gegen semantische Verwechslungen zu schützen, die etwa unter dem Motto »in Würde sterben« drohen könnten. Die Einsicht, dass selbstbestimmtes Leben nur in einem unterstützenden sozialen Umfeld gedeihen kann, ist keineswegs nur für Menschen mit Behinderungen relevant. Und die diskriminierungsfreie Gewährleistung von Menschenrechten im Gesundheitswesen verlangt sowohl Offenheit für die Berücksichtigung neuer Diskriminierungsmerkmale - etwa aufgrund von Alter oder aufgrund von sexueller Orientierung - als auch Sensibilität für versteckte Formen von Diskriminierung struktureller Art. Eine der größten und schwierigsten aktuellen »Baustellen « betrifft die Klärung der extraterritorialen Staatenpflichten im Kontext des Rechts auf Gesundheit. Zwischen dem Handlungsdruck, der aus praktischen Imperativen der Flüchtlingssolidarität oder der Entwicklungszusammenarbeit folgt, und den bislang etablierten rechtlichen Standards zu extraterritorialen menschenrechtlichen Verpflichtungen der Staaten klafft eine breite Kluft. Überbrücken lässt sie sich offensichtlich nur durch verstärkte Koordination und Kooperation der Staaten auch im Bereich des Rechts auf Gesundheit.

Menschenrechte im Gesundheitswesen sind ein vergleichsweise neues Gebiet. Die Themen, die dabei zur Debatte stehen, resultieren einerseits aus aktuellen Herausforderungen: ambivalenten technologischen Entwicklungen, grenzüberschreitenden Migrationsbewegungen oder Qualitätssprüngen innerhalb von Globalisierungsprozessen; sie sind andererseits aber zugleich altbekannt. Auch die normativen Orientierungen, die die Menschenrechte geben, weisen bei aller Modernität des Menschenrechtsansatzes zugleich manche Kontinuitäten zu vormodernen normativen Traditionen auf. Dass 
die Menschenwürde mit rechtlich gestützten Ansprüchen auf individuelle Autonomie verknüpft wird, dürfte zwar ziemlich neu sein. Die Idee, dass dem Menschen eine unbedingt zu respektierende Würde zukommt, lässt sich gleichwohl bis in die Grunddokumente unterschiedlicher Religionen, Kulturen und Philosophien zurückverfolgen. Systematische Antidiskriminierungsgesetzgebung ist ein spezifisch modernes Projekt. Der dahinter stehende Gerechtigkeitsimpuls ist zugleich aber uralt.

Menschenrechte weisen vielfach sehr spezifische »technische« Züge auf, die das Terrain der Fachleute bilden, aber um ihre Grundlage zu verstehen, braucht es nicht viel mehr als politischen common sense. Bei allem Streit über komplizierte Fragen sollte dies nicht vergessen werden. denn die Rechte, die dem Menschen als Menschen zukommen, hätten ansonsten von vornherein keine Chance auf Realisierung.

\section{LITERATUR}

Aichele, Valentin (Hg.) (2013): Das Menschenrecht auf gleiche Anerkennung vor dem Recht. Artikel 12 der UN-Behindertenrechtskonvention, Baden-Baden: Nomos.

Arendt, Hannah (1949): „Es gibt nur ein einziges Menschenrecht «, in: Die Wandlung 4 (1949), 754-770.

Arendt, Hannah (1974): Elemente und Ursprünge totaler Herrschaft, 2. Auflage, München u.a.: Piper.

Baer, Susanne (2009): » Sexuelle Selbstbestimmung«? Zur internationalen Rechtslage und denkbaren Konzeptionen von Recht gegen geschlechtsbezogene Diskriminierungen«, in: Lohrenscheit (2009), 89-118.

Bielefeldt, Heiner (1998): Philosophie der Menschenrechte, Darmstadt: Wissenschaftliche Buchgesellschaft.

Bielefeldt, Heiner (2003): Symbolic Representation in Kant's Practical Philosophy, Cambridge: Cambridge University Press.

Bielefeldt, Heiner (2011): Auslaufmodell Menschenwürde? Warum sie in Frage steht und warum wir sie verteidigen müssen, Freiburg: Herder.

Bielefeldt, Heiner (2013): »Misperceptions of Freedom of Religion or Belief«, in: Human Rights Quarterly 35 (2013), 33-68. 
Bielefeldt, Heiner/Brugger, Winfried/Dicke, Klaus (Hg.) (1992): Würde und Recht des Menschen. Festschrift für Johannes Schwartländer zum 70. Geburtstag, Würzburg: Königshausen \& Neumann.

Bielefeldt, Heiner/Ghanea, Nazila/Wiener, Michael (2016): Freedom of Religion or Belief. An International Law Commentary, Oxford: Oxford University Press.

Brunner, Otto/Conze, Werner/Koselleck, Reinhard (Hg.) (1978): Geschichtliche Grundbegriffe. Historisches Lexikon zur politisch-sozialen Sprache in Deutschland, Band 7, Stuttgart: Klett-Cotta.

Deutsches Institut für Menschenrechte (Hg.) (2005): Die »General Comments« zu den VN-Menschenrechtsverträgen. Deutsche Übersetzung und Kurzeinführungen, Baden-Baden: Nomos.

Deutsches Institut für Menschenrechte/Deile, Volkmar/Hutter, Franz-Josef/ Kurtenbach, Sabine/Tessmer, Carsten (Hg.) (2006): Jahrbuch Menschenrechte 2006. Freiheit in Gefahr - Strategien für die Menschenrechte, Frankfurt/M.: Suhrkamp.

Dicke, Klaus (1992): »Die der Person innewohnende Würde«, in: Bielefeldt et al. (1992), 161-182.

Di Fabio, Udo (2008): »Menschenrechte in unterschiedlichen Kulturräumen«, in: Nooke et al. (2008), 63-97.

Forst, Rainer (2007): Das Recht auf Rechtfertigung. Elemente einer konstruktivistischen Theorie der Gerechtigkeit, Frankfurt/M.: Suhrkamp.

Gerhardt, Volker (2004): »Redebeitrag«, in: Nationaler Ethikrat. Wortprotokoll. Niederschrift über den öffentlichen Teil der Sitzung vom 27. Mai 2004, 9.

Gewirth, Alan (1992): »Human Dignity as the Basis of Rights«, in: Meyer et al. (1992), 10-46.

Giegerich, Thomas/Zimmermann, Andreas (Hg.) (2008): Wirtschaftliche, soziale und kulturelle Rechte im globalen Zeitalter, Berlin: Duncker \& Humblot.

Graumann, Sigrid (2009): Assistierte Autonomie. Von einer Behindertenpolitik der Wohltätigkeit zu einer Politik der Menschenrechte. UtrechtPublications of the Department of Philosophy Utrecht University.

Habermas, Jürgen (2005): Die Zukunft der menschlichen Natur. Auf dem Weg zu einer liberalen Eugenik?, Frankfurt/M.: Suhrkamp. 
Habermas, Jürgen (2010): „Das utopische Gefälle. Das Konzept der Menschenwürde und die realistische Utopie der Menschenrechte«, in: Blätter für deutsche und internationale Politik 8 (2010), 43-53.

Hegselmann, Rainer/Merkel, Reinhard (Hg.) (1991): Zur Debatte über Euthanasie. Beiträge und Stellungnahmen, Frankfurt/M.: Suhrkamp.

Hunt, Paul/Backman, Gunilla (2008): »Health systems and the right to the hightest attainable standard of health «, in: Health and Human Rights 10, 1 (2008), 81-92.

Huhle, Rainer (Hg.) (2009): Human Rights and History: A Challenge for Education, Berlin: Fata Morgana Verlag.

Kjaerum, Morten (2009): »Human Rights and their History from a European Perspective«, in: Huhle (2009), 19-24.

Krajewski, Markus (2010): »Grenzüberschreitende Patientenmobilität in Europa zwischen negativer und positiver Integration der Gesundheitssysteme«, in: Europarecht (EuR) 45 (2010), 165-188.

Krennerich, Michael (2006): „Soziale Rechte sind Freiheitsrechte! Plädoyer für ein freiheitliches Verständnis wirtschaftlicher, sozialer und kultureller Rechte«, in: Deutsches Institut für Menschenrechte (2006), 5766.

Krennerich, Michael (2013): Soziale Menschenrechte. Zwischen Recht und Politik. Schwalbach/Ts.: Wochenschau-Verlag.

Lohrenscheit, Claudia (Hg.) (2009): Sexuelle Selbstbestimmung als Menschenrecht, Baden-Baden: Nomos.

London, Leslie (2008): »What is a human-rights based approach to health and does it matter?«, in: Health and Human Rights 10, 1 (2008), 65-80.

Meyer, Michael J./Parent, William A. (Hg.) (1992): The Constitution of Rights. Human Dignity and American Values, Ithaca/London: Cornell University Press.

Morsink, Johannes (1999): The Universal Declaration of Human Rights: Origins, Drafting and Intent, Philadelphia: University of Pennsylvania Press.

Müller, Ernst (Hg.) (1954): Ausgewählte Werke, Stuttgart u.a.: Europäischer Buchclub.

Müntefering, Franz (2014): »Freiheit ist auch die Freiheit des anderen«, in: SZ vom 3. Januar 2014, Online: www.sueddeutsche.de/leben/debatteum-sterbehilfe-gefaehrliche-melodie-1.1854960-2 [18.11.2015]. 
Mylius, Maren/Bornschlegel, Wiebke/Frewer, Andreas (Hg.) (2011): Medizin für »Menschen ohne Papiere«. Menschenrechte und Ethik in der Praxis des Gesundheitswesens, Medizin und Menschenrechte, Bd. 5, Göttingen: V\&R unipress.

Nooke, Günter/Lohmann, Georg/Wahlers, Gerhard (Hg.) (2008): Gelten Menschenrechte universal?, Freiburg: Herder.

Nussbaum, Martha C. (2008): Liberty of Conscience. In Defense of America's Tradition of Religious Equality, New York: Basic Books.

Pöschl, Viktor/Kondylis, Panajotis (1978): »Artikel >Würde««, in: Brunner et al. (1978), 637-677.

Reuter, Hans-Richard (Hg.) (1999): Ethik der Menschenrechte. Zum Streit um die Universalität einer Idee I., Tübingen: Mohr Siebeck.

Riedel, Eibe H. (1986): Theorie der Menschenrechtsstandards, Berlin: Duncker \& Humblot.

Riedel, Eibe H. (2008): »Zur Durchsetzung wirtschaftlicher, sozialer und kultureller Rechte im Völkerrecht«, in: Giegerich et al. (2008), 71-93.

Sandkühler, Hans Jörg (Hg.) (2007a): Menschenwürde. Philosophische, theologische und juristische Analysen, Frankfurt/M. u.a.: Lang.

Sandkühler, Hans Jörg (2007b): »Menschenwürde und die Transformation moralischer Rechte in positives Recht«, in: Sandkühler (2007a), 57-86.

Schiller, Friedrich (1954): »Über Anmut und Würde«, in: Müller (1954), 193-258.

Schneider, Jakob (2004): Die Justitiabilität wirtschaftlicher, sozialer und kultureller Menschenrechte, Berlin: Deutsches Institut für Menschenrechte.

Schwartländer, Johannes (Hg.) (1978): Menschenrechte. Aspekte ihrer Begründung und Verwirklichung, Tübingen: Attempto.

Spaemann, Robert (1996): Personen. Versuche über den Unterschied zwischen »etwas « und »jemand «, Stuttgart: Klett-Cotta.

Stellmacher, Jost/Sommer, Gert/Brähler, Elmar (2007): »Human Rights: knowledge, importance and support - results of two representative studies in Germany«, in: Peace and Conflict. Journal of Peace Psychology 11 (2007), 267-292.

Vögele, Wolfgang (1999): »Christliche Elemente in der Begründung von Menschenrechten und Menschenwürde im Kontext der Entstehung der Vereinten Nationen«, in: Reuter (1999), 103-133. 
Willoweit, Dietmar (1992): »Die Veräußerung der Freiheit. Über den Unterschied von Rechtsdenken und Menschenrechtsdenken «, in: Bielefeldt et al. (1992), 255-268. 


\title{
Das Menschenrecht auf Gesundheit
}

\author{
Grundzüge eines komplexen Rechts
}

MICHAEL KRENNERICH

\section{EINLEITUNG ${ }^{1}$}

Menschenrechte, so wie sie im Völkerrecht verankert sind, beziehen sich primär auf das Verhältnis zwischen den Menschen als Rechteinhabern und dem Staat als vorrangigem Pflichtenträger. Die Grundidee eines solchen Menschenrechts auf Gesundheit ist, dass der Staat die Gesundheit der Menschen nicht beeinträchtigt, diese vor Eingriffen schützt und Maßnahmen ergreift, damit die Menschen gesunde Lebens- und Arbeitsbedingungen vorfinden und sie vor allem Zugang zu einer angemessenen Gesundheitsversorgung haben.

1 Der Beitrag beruht auf einem Hintergrundpapier zur EFI-Konferenz »Sich für die Gesundheit stark machen! Menschenrechtliches Empowerment unter den Bedingungen von Armut « am 26./27. März 2015 und wurde in leicht modifizierter Form in der Zeitschrift für Menschenrechte (zfmr) 9, 2 (2015) abgedruckt. 


\section{VöLKERRECHTLICHE VERANKERUNG DES MENSCHENRECHTS AUF GESUNDHEIT}

Der Beitrag beginnt mit der Frage, wo das Recht auf Gesundheit im geltenden Völkerrecht verankert ist. Die Antwort lautet: an vielen Stellen und auf unterschiedliche Weise. Wohl oder übel müssen wir zunächst einen kleinen Pfad durch das Dickicht völkerrechtlicher Dokumente und Abkommen schlagen, in denen das Menschenrecht auf Gesundheit - hier weiter, dort enger gefasst - niedergelegt ist. Ausgangspunkt ist die zweite Hälfte der 1940er Jahre, als nach dem Zweiten Weltkrieg, anknüpfend an entsprechende historische Vorläufer und zeitgenössische Vorarbeiten, der moderne internationale Menschenrechtsschutz entstand und das Menschenrecht auf Gesundheit im Rahmen der Vereinten Nationen verankert wurde.

So legte die Weltgesundheitsorganisation (World Health Organization, WHO) bereits in der Präambel ihrer Verfassung von 1946 fest, dass es ein grundlegendes Recht eines jeden Menschen auf »enjoyment of the highest attainable standard of health « gäbe. Dabei definierte die WHO Gesundheit in einem umfassenden und anspruchsvollen Sinn als »state of complete physical, mental and social wellbeing and not merely the absence of disease or infirmity. ${ }^{2}$ Die WHO löste sich damit von einem rein biomedizinischen Verständnis (und den allermeisten Alltagsvorstellungen) von Gesundheit als Freisein von körperlichen und psychischen Erkrankungen und Beeinträchtigungen $^{3}$ und entfachte eine rege Fachdiskussion, nicht nur unter Medizinern. Zum einen wurde das Konzept von Gesundheit als vollkommenes Wohlbefinden ${ }^{4}$ häufig als utopisch kritisiert. ${ }^{5}$ Zum anderen blieb stark umstritten, worin ein solches wellbeing besteht. ${ }^{6}$

2 Constitution of the World Health Organization: www.who.int/governance/eb/ who_constitution_en.pdf [01.07.2015].

3 Vgl. Franke (2006), 32.

4 Möglicherweise wäre es sinnvoller, »complete wellbeing « eher im Sinne eines »umfassenden« anstatt eines »vollständigen« Wohlbefindens $\mathrm{zu}$ verstehen. Alternativ wird der Begriff des wellbeing mitunter auch mit »Wohl« oder »Wohlergehen« übersetzt.

5 Statt vieler: Venkatapuram (2011), 66.

6 Siehe etwa Dodge et al. (2012). 
Obwohl die WHO-Präambel aufgrund ihres nicht-bindenden Charakters keine Rechtsquelle im engeren Sinne für das Recht auf Gesundheit darstellt, ${ }^{7}$ wird die WHO-Definition im Menschenrechtsdiskurs immer wieder herangezogen. ${ }^{8}$ Interessante Aspekte für die Debatte ergeben sich nicht nur dadurch, dass der Ansatz die Verwobenheit körperlicher, geistig-seelischer und sozialer Aspekte von Gesundheit hervorhebt. Betont wird auch die dem Wohlbefinden inhärente - subjektive Komponente von Gesundheit. Die Bestimmung von Gesundheit als wellbeing ist, so verstanden, nicht mehr nur eine Sache von Expertinnen und Experten. Andererseits: Nicht jeder Mensch, der sich wohlfühlt, ist - medizinisch betrachtet - gesund, und nicht jeder, der sich schlecht fühlt, gilt als krank. So besteht die Gefahr, dass das biomedizinische Profil von Gesundheit, das für das Gesundheitswesen im engen Sinne unabdingbar ist (und das die WHO nicht völlig aufgibt), an Trennschärfe verliert.

Auch die Allgemeine Erklärung der Menschenrechte (AEMR) von 1948 war, da es sich »nur « um eine Erklärung der UN-Vollversammlung handelt, ursprünglich nicht völkerrechtlich verbindlich. Jedoch hat die in über 300 Sprachen übersetzte AEMR im Laufe der Zeit eine große moralische, politische und zumindest mittelbar auch rechtliche Wirkkraft entwickelt. Ihre Rechtswirkung beruht darauf, dass sie allgemeine Rechtsprinzipien und völkergewohnheitsrechtlich anerkannte Garantien des Menschenrechtsschutzes enthält. Gut begründet ist auch die Auffassung, dass die AEMR das Ziel der - für die Mitgliedsstaaten der Vereinten Nationen verbindlichen - UN-Charta konkretisiert, die Achtung der Menschenrechte und Grundfreiheiten für alle Menschen ohne Unterschied zu fördern und zu festigen. $^{9}$

In der AEMR findet sich das Menschenrecht auf Gesundheit indes nicht, wie zu erwarten wäre und in der Menschenrechtskommission diskutiert wurde, ${ }^{10}$ als eigenständiger Artikel. Vielmehr wurde es, auch um die AEMR knapp und bündig zu halten, als Teil des Rechts auf einen angemessenen Lebensstandard verankert, der jedem Menschen Gesundheit und

7 Vgl. Hestermeyer (2007), 113.

8 Vgl. etwa UNICEF (2002), 344 und Freeman et al. (2012), 315.

9 Zur Rechtsnatur der AEMR siehe u.a. Nettelsheim (2009).

10 Vgl. Morsink (1999), 192-199. Siehe auch die Dokumentensammlung von Schabas (2013). 
Wohlbefinden (health and well being) gewährleistet, einschließlich Nahrung, Kleidung, Unterkunft, ärztlicher Versorgung und notwendiger sozialer Leistungen (Art. 25 Abs. 1). Im Unterschied zur Präambel der WHO-Verfassung unterscheidet die AEMR dem Wortlaut nach zwar zwischen Gesundheit und Wohlbefinden, verdeutlicht aber gleichzeitig, dass beide eng zusammenhängen. Im Hinblick auf das Recht auf Gesundheit, das in der AEMR nicht klar von den anderen Komponenten des Rechts auf angemessenen Lebensstandard abgegrenzt wird, ist festzuhalten, dass die AEMR die medizinische Versorgung zwar als einen zentralen, aber nicht als einzigen Faktor dieses Rechts ausweist. Auch die soziale Sicherheit, gerade im Falle von Krankheit und Invalidität, sowie der Zugang zu gesunden Lebensbedingungen (Nahrung, Wohnen etc.) spielen eine wichtige Rolle.

Eigenständig und völker(vertrags-)rechtlich verbindlich wurde das Recht auf Gesundheit später im Internationalen Pakt über wirtschaftliche, soziale und kulturelle Menschenrechte (kurz: UN-Sozialpakt) von 1966 verankert, der 1976 in Kraft trat. Der UN-Sozialpakt ist das grundlegende UN-Menschenrechtsabkommen zu den wirtschaftlichen, sozialen und kulturellen Rechten (kurz: WSK-Rechte oder soziale Menschenrechte), einschließlich des Rechts auf Gesundheit. In dem Abkommen erkennen die mittlerweile 164 Vertragsstaaten des Paktes (Stand: 12.10.2015) das Recht eines jeden Menschen auf ein für ihn erreichbares Höchstmaß an körperlicher und geistiger Gesundheit an (Art. 12 Abs. 1).

Hier sind zwei Aspekte hervorzuheben: Zum einen rückt der UNSozialpakt von dem Verständnis eines Rechtes, gesund zu sein, ab. ${ }^{11}$ Ein solches scheint in der Präambel der WHO-Verfassung, die von einem $\mathrm{Zu}$ stand des vollständigen körperlichen, geistigen und sozialen Wohlbefindens spricht, noch angelegt zu sein. Damit wird (noch) stärker betont, dass der Staat allein die Gesundheit eines Menschen nicht garantieren kann. Zum anderen bezieht sich im UN-Sozialpakt das erreichbare Höchstmaß nur auf die körperliche und geistige Gesundheit, nicht aber etwa auf »social wellbeing « (wie der ursprüngliche Kommissionsentwurf noch vorsah) oder gar auf »moral wellbeing « (wie bei der Ausarbeitung des Abkommens seinerzeit die Delegationen aus Afghanistan und den Philippinen vorschlu-

11 Darauf weist ausdrücklich auch der UN-Ausschuss für wirtschaftliche, soziale und kulturelle Rechte in seinem Kommentar zum Recht auf Gesundheit hin; siehe CESCR E/C.12/2000/4, 11. August 2000, Ziff. 4 und 8. 
gen). ${ }^{12}$ Die sozialen Verhältnisse stellen demzufolge eher Bestimmungsgründe und Möglichkeitsräume für Gesundheit dar als deren definitorischen Bestandteil.

Auch die in der nicht-abschließenden Liste des UN-Sozialpaktes angeführten Maßnahmen zur Umsetzung des Rechts rücken die körperliche (und geistige) Gesundheit in den Fokus, freilich unter Einbeziehung von Kontextbedingungen, welche die Gesundheit fördern oder beeinträchtigen. Die Maßnahmen zielen auf die Verminderung von Totgeburten und Kindersterblichkeit ab, auf die gesunde Entwicklung des Kindes, gesunde Umwelt- und Arbeitsbedingungen, auf die Vorbeugung, Behandlung und Bekämpfung epidemischer, endemischer, Berufs- und sonstiger Krankheiten sowie auf den Zugang der Menschen zu medizinischen Einrichtungen und ärztlicher Betreuung (Art. 12 Abs. 2). Überschneidungen ergeben sich dabei mit dem Recht auf gerechte und faire Arbeitsbedingungen und dem Recht auf soziale Sicherheit, die beide in eigenständigen Artikeln verankert sind.

Weitere UN-Menschenrechtsabkommen umfassen ebenfalls das Recht auf Gesundheit oder einzelne Aspekte davon, und zwar bezüglich besonderer Problembereiche oder besonders benachteiligter oder bedürftiger Bevölkerungsgruppen. Die UN-Antirassismuskonvention von 1966 (seit 1969 in Kraft) verankert das diskriminierungsfrei zu gewährende Recht auf öffentliche Gesundheitsfürsorge, ärztliche Betreuung, soziale Sicherheit und soziale Dienstleistungen (Art. 5).

Die UN-Konvention zur Beseitigung der Diskriminierung der Frau (CEDAW) von 1979 (seit 1981 in Kraft), die bis heute immerhin 189 Staaten ratifiziert haben, sieht - verstreut in dem Abkommen - zahlreiche Maßnahmen vor, um das diskriminierungsfreie Recht auf Gesundheit umzusetzen, und zwar nicht nur im Gesundheitswesen (Art. 12), sondern auch im Bereich der gesundheitsbezogenen Bildung, am Arbeitsplatz, bei der Familienplanung, in ländlichen Gebieten sowie während der Schwangerschaft, der Entbindung und der Stillzeit. ${ }^{13}$ Etliche andere Rechte betreffen soziale Bestimmungsgründe der Gesundheit. ${ }^{14}$ Indem das Abkommen die Geschlechter- und Gender-Dimensionen von Gesundheit aufzeigt, ermöglicht

12 Vgl. Saul et al. (2014), 980. Siehe auch Tobin (2012), 125.

13 CEDAW, Art. 10 (h), Art. 11 Abs. 1 (f) und Abs. 2 (d), Art. 14 Abs. 2 (b).

14 Vgl. CEDAW A/54/38/REV I., 2. Februar 1999; siehe auch WHO (2008). 
es, Menschenrechtsverletzungen festzustellen, die sonst vielleicht verborgen geblieben wären, und menschenrechtspolitische Maßnahmen einzufordern, die sonst möglicherweise nicht (so umfassend) ergriffen würden. Dazu zählen etwa Maßnahmen in Bezug auf die reproduktive und sexuelle Gesundheit. $^{15}$

Die UN-Kinderrechtskonvention von 1989 (seit 1990 in Kraft), die inzwischen 196 Vertragsparteien aufweist und - mit Ausnahme der USA von allen Staaten weltweit ratifiziert wurde, erkennt das Recht des Kindes »auf ein erreichbares Höchstmaß an Gesundheit an sowie auf Inanspruchnahme von Einrichtungen zur Behandlung von Krankheiten und zur Wiederherstellung der Gesundheit« (Art. 24 Abs. 1). Das Abkommen benennt (nicht abschließend) vielfältige Maßnahmen, um die volle Verwirklichung des Rechts auf Gesundheit sicherzustellen: u.a. die Verringerung der Säuglings- und Kindersterblichkeit, die Sicherstellung ärztlicher Hilfe und Gesundheitsfürsorge für Kinder, die Bekämpfung von Unter- und Fehlernährung, die Gesundheitsfürsorge für Mütter sowie verschiedene Aspekte der Aufklärung im Bereich der Gesundheit, der Hygiene, des Stillens, der Unfallverhütung und der Familienplanung (Art. 24). Zugleich sieht die UN-Kinderrechtskonvention Schutzmaßnahmen gegen gesundheitsschädliche wirtschaftliche Ausbeutung von Kindern und gegen den Gebrauch von Suchtstoffen durch Kinder vor (Art. 32 Abs. 1, Art. 33). Besondere Schutzpflichten ergeben sich auch aus der staatlichen Unterbringung körperlich und/oder geistig erkrankter Kinder (Art. 25). Weiterhin geht das Abkommen auf die besonderen Bedürfnisse von Kindern mit Behinderungen ein, deren Zugang zu Gesundheitsdiensten sicherzustellen ist (Art. 23).

Die Rechte von Menschen mit Behinderungen, einschließlich des Rechts auf Gesundheit, wurden wiederum eigens in der UN-Behindertenrechtskonvention von 2006 (seit 2008 in Kraft) konkretisiert und ausdifferenziert. Mit dieser erkennen inzwischen 159 Vertragsparteien (Stand: 12.10.2015) das Recht behinderter Menschen auf das erreichbare Höchstmaß an Gesundheit an, und zwar ohne Diskriminierungen aufgrund ihrer Beeinträchtigungen. Auch die UN-Behindertenrechtskonvention benennt, wiederum nicht abschließend, zahlreiche Maßnahmen zur vollen Umsetzung des Rechtes. Diese zielen darauf ab, Diskriminierungen beim Zugang zur Gesundheitsversorgung und - was oft übersehen wird - zu Kranken-

15 Vgl. Freeman et al. (2012), 320-323. 
und Lebensversicherungen abzubauen, und bezwecken, den spezifischen Gesundheitsbedürfnissen von Menschen mit Behinderung Rechnung zu tragen (Art. 25). Indem die Behindertenrechtskonvention von einem »diversity-Ansatz« ausgeht, der körperliche und geistige Beeinträchtigung als Bestandteil menschlicher Normalität versteht, und indem sie auf eine »Enthinderung « der Menschen abstellt, die mit vielfältigen gesellschaftlichen Barrieren konfrontiert sind, überwindet sie eindrucksvoll einen rein medizinischen »Defizit-Ansatz« von Behinderung. Zugleich nimmt sie aber körperliche und geistige Beeinträchtigungen ernst genug, um besonderen Handlungsbedarf für eine assistierte Autonomie ${ }^{16}$ im Gesundheits- und in anderen Lebensbereichen auszumachen. So stark wie kein anderes Abkommen ist die Behindertenrechtskonvention vom Verständnis der Gesundheit als »Befähigung « geprägt. ${ }^{17}$

Die bislang genannten Abkommen machen dem Wortlaut nach keinen Unterschied zwischen Menschen unterschiedlicher Staatsangehörigkeit. In der Praxis der jeweiligen Staaten jedoch genießen Ausländerinnen und Ausländer mitunter nicht dieselben Ansprüche auf eine gesundheitliche Versorgung wie Inländerinnen und Inländer. Deutlich wird diese Problematik in der - gleichwohl nur von 48 meist afrikanischen und lateinamerikanischen Staaten ratifizierten (Stand 12.10.2015) - UN-Wanderarbeiterkonvention von 1990 (seit 2003 in Kraft), die sich der Rechte von Arbeitsmigrantinnen und -migranten annimmt und entsprechende Einschränkungen beinhaltet.

Die Gleichbehandlung mit den Staatsangehörigen des betreffenden Staates beschränkt sich dort auf die dringende ärztliche Versorgung:

»Wanderarbeitnehmer und ihre Familienangehörigen haben das Recht, jede ärztliche Versorgung, die für die Erhaltung ihres Lebens oder die Vermeidung einer nicht wiedergutzumachenden Schädigung ihrer Gesundheit dringend erforderlich ist, auf

16 Siehe zum Konzept der »assistierten Freiheit« die Studie von Graumann (2011).

17 Ein Befähigungsansatz (capabilities approach), wie ihn beispielsweise, angelehnt an Amartya Sen, Venkatapuram in Bezug auf Gesundheit vertritt, konzeptionalisiert Gesundheit als »a meta-capability, the capability to achieve a cluster of basis capabilities to be and do things that reflect a life worthy of equal human dignity.« Vgl. Venkatapuram (2011), 71. 
der Grundlage der Gleichbehandlung mit den Staatsangehörigen des betreffenden Staates zu erhalten.«(Art. 28, Satz 1)

Gemäß der Konvention darf Arbeitsmigranten auch dann nicht die dringliche Versorgung verweigert werden, wenn sie sich »irregulär « in dem Land aufhalten oder »irregulär« dort arbeiten (Art. 28, Satz 2). Einen gleichbehandelnden allgemeinen Zugang zu Sozial- und Gesundheitsdiensten genießen Wanderarbeitnehmer und ihre Familien aber nur, »sofern die erforderlichen Voraussetzungen für die Inanspruchnahme erfüllt sind« (Art. 43 e). So ist die Wanderarbeiterkonvention ein ambivalentes Dokument: Einerseits betont sie den wichtigen Rechtsanspruch von Arbeitsmigrantinnen und -migranten auf eine medizinische Grundversorgung, andererseits bleibt sie einer Rechtspraxis verhaftet, die eine umfassende medizinische Behandlung vom Aufenthaltsstatus abhängig macht.

Bliebe zu erwähnen, dass auch verschiedene regionale Menschenrechtsabkommen das Recht auf Gesundheit beinhalten. Im Rahmen des Europarates ist hier gerade die Europäische Sozialcharta in ihrer ursprünglichen (1961/1965) und ihrer revidierten (1996/1998) Fassung zu nennen, die eine wirksame Ausübung des Rechtes auf Schutz der Gesundheit zu gewährleisten sucht (Art. 11). Das Zusatzprotokoll zur Amerikanischen Menschenrechtskonvention (Protokoll von Salvador) von 1988, seit 1999 in Kraft, beinhaltet ebenfalls ein Recht auf Gesundheit, das dort im Sinne der WHO als ein Höchstmaß an körperlichem, geistigem und sozialem Wohlbefinden definiert wird (Art. 10 Abs. 1). Die Afrikanische Charta der Menschenrechte und Rechte der Völker (Banjul-Charta) von 1981, die 1986 in Kraft trat, garantiert wiederum ein erreichbares Höchstmaß an körperlicher und geistiger Gesundheit (Art. 16), während beispielsweise die 1999 in Kraft getretene Afrikanische Kinderrechtscharta von 1990 das Recht auf »best attainable state of physical, mental and spiritual health « vorsieht (Art. 14 Abs. $1)$. 


\section{GRundzüGE des MenschenReChtS AUF GESUNDHEIT}

Angesichts der vielen Rechtsquellen ist eine allgemeine Auslegung des Menschenrechts auf Gesundheit kein leichtes Unterfangen. Ein geeigneter Ausgangspunkt, um die Grundzüge dieses Rechts zu erfassen, ist der UN-Sozialpakt, der das grundlegende UN-Menschenrechtsabkommen zu wirtschaftlichen, sozialen und kulturellen Rechten darstellt und daher im Fokus des vorliegenden Beitrags steht. Hilfreich sind diesbezüglich die Interpretationsvorgaben durch den 1988 gegründeten UN-Ausschuss für wirtschaftliche, soziale und kulturelle Menschenrechte, der den UN-Sozialpakt überwacht. Der Ausschuss veröffentlichte im Jahr 2000 einen »Allgemeinen Kommentar « zum Recht auf Gesundheit ${ }^{18}$ und nimmt im Rahmen des Staatenberichtsverfahrens regelmäßig Stellung zur Umsetzung der sozialen Menschenrechte durch die Vertragsstaaten des UN-Sozialpaktes. Zwar sind die Kommentare und Empfehlungen des Ausschusses nicht rechtsverbindlich, doch bieten sie eine weithin anerkannte Orientierungshilfe bei der zeitgemäßen Auslegung einzelner WSK-Rechte. Auch andere UN-Menschenrechtsvertragsorgane sowie UN-Sonderberichterstatterinnen und -berichterstatter nehmen darauf Bezug. ${ }^{19}$

Das Menschenrecht auf Gesundheit, so wie es im UN-Sozialpakt verankert ist, berechtigt jeden Menschen, ein für ihn erreichbares Höchstmaß an körperlicher und geistiger Gesundheit zu erreichen, um ein Leben in Würde zu führen. Dies wird, wie gesagt, nicht einfach als eine rechtliche Garantie verstanden, gesund zu sein. Eine solche Garantie könnte kein Staat abgeben, schon allein, weil die Gesundheit von Faktoren abhängt, die der Staat nicht zu kontrollieren vermag oder mit gutem, menschenrechtlichem Grund nicht zu kontrollieren hat. Man denke hier etwa an die genetische Veranlagung der Menschen. Obwohl bereits jetzt die technischen Möglichkeiten -

18 CESCR E/C.12/2000/4, 11. August 2000. Eine deutsche Übersetzung findet sich in Deutsches Institut für Menschenrechte (2005), 285-313 sowie im Anhang des vorliegenden Bandes.

19 Die entsprechenden Dokumente sind allesamt auf dem Menschenrechtsportal des UN-Hochkommissariats für Menschenrechte abrufbar: Vgl. www.ohchr.org [01.10.2015]. 
die »Werkzeuge zur Menschenzüchtung ${ }^{20}{ }^{2}$ - verfügbar sind, um krankheitsvermeidende Genkorrekturen vorzunehmen, stellt die (zumal staatliche) Manipulierung des Erbgutes bislang (noch) ein Tabu dar. Selbst eine gesunde Lebensweise, sofern darüber überhaupt Einvernehmen herrscht, lässt sich nicht aufoktroyieren, zumindest nicht auf eine Weise, die der Menschenwürde und damit der freiheitlichen Eigenverantwortung der Menschen Rechnung trägt. Gleichwohl gibt es Möglichkeiten des Staates, gerade die politischen, sozioökonomischen und ökologischen Bedingungen von Gesundheit mitzugestalten. In diesem Sinne hebt das Menschenrecht auf Gesundheit zum einen darauf ab, dass die Menschen nicht in ihrer Gesundheit beeinträchtigt werden. Zum anderen müssen die Voraussetzungen dafür geschaffen werden, dass alle Menschen Zugang zu einer angemessenen Gesundheitsversorgung haben und dass sie in eigenverantwortlicher Selbstbestimmung gesund leben und arbeiten können. Dazu zählen dann auch gesundheitsrelevante Informationen und Aufklärung.

Das Recht auf Gesundheit umfasst also zunächst die Freiheit, über die eigene Gesundheit und den eigenen Körper selbst zu bestimmen, sowie das Recht, frei von Eingriffen in die Gesundheit zu bleiben. Hier ergeben sich bereits offenkundige Überschneidungen u.a. mit den Rechten auf Leben, auf Privatleben, inklusive individuelle Selbstbestimmung und körperliche wie geistige Unversehrtheit, ${ }^{21}$ sowie mit dem Verbot der Folter und unmenschlicher oder erniedrigender Behandlung oder Strafe. Zugleich zeichnen sich an dieser Stelle bereits einige Problemfelder im Gesundheitswesen ab: Wie steht es beispielsweise um - aus medizinischer oder staatlicher Sicht - notwendige Zwangsbehandlungen gegen den Patientenwillen ${ }^{22}$ Oder: Wie kann die gegebenenfalls assistierte Selbstbestimmung von Menschen aussehen, die in ihrer Willensbildung und Willensäußerung stark ein-

20 Bahnsen (2015), 1.

21 Der Rechtsprechung des EGMR zufolge umfasst das Recht auf Privatleben (right to privacy) auch die physische und psychische Integrität sowie die individuelle Selbstbestimmung hinsichtlich des Verfügungsrechts über den eigenen Körper; vgl. Kälin/Künzli (2008), 437-439.

22 Neben der umfassenden Fachliteratur zu dem Thema verweist beispielsweise ein jüngerer FAZ-Artikel von Platthaus (2015) journalistisch auf die Problematik. 
geschränkt sind, etwa Demenzkranke im fortgeschrittenen Stadium? ${ }^{23}$ Der Autonomiebegriff steht hier im Zentrum der Diskussion. ${ }^{24}$

Das Recht auf Gesundheit fordert weiterhin, soziale Bedingungen $\mathrm{zu}$ schaffen oder zu erhalten, damit Menschen ein gesundes Leben führen können. Nach Ansicht des UN-Ausschusses für WSK-Rechte umfassen diese etwa den Zugang zu sauberem Trinkwasser und sanitärer Versorgung, unbedenkliche Nahrung und Unterkunft, gesunde Arbeits- und Umweltbedingungen, gesundheitsrelevante Aufklärung, auch über Sexualgesundheit und reproduktive Gesundheit, sowie die Beteiligung der Bevölkerung an gesundheitsbezogenen Entscheidungen. ${ }^{25}$ Während sich diesbezüglich Überschneidungen u.a. mit den Rechten auf angemessene Nahrung, sauberes Trinkwasser und gerechte Arbeitsbedingungen ergeben, beinhaltet das Recht auf Gesundheit darüber hinaus eigens einen Anspruch auf eine präventive, kurative und palliative ${ }^{26}$ Gesundheitsversorgung, die jedem Menschen gewährleisten soll, ein für ihn erreichbares Höchstmaß an Gesundheit zu erlangen. Im Hinblick auf die Gesundheitsfürsorge und die ärztliche Betreuung verwendet der UN-Ausschuss für WSK-Rechte - ähnlich wie bei anderen sozialen Menschenrechten - die Kategorien der Verfügbarkeit (availability), des offenen Zugangs (accessibility), der Annehmbarkeit (acceptability) sowie der Qualität (quality), um das Recht zu konkretisieren. ${ }^{27}$ Im Sinne der Verfügbarkeit müssen funktionierende Einrichtungen der Gesundheitsfürsorge sowie der medizinischen bzw. ärztlichen Behandlung und Betreuung vorhanden sein. Obwohl deren konkrete Beschaffenheit von vielen Faktoren - insbesondere vom Entwicklungsstand und den Ressourcen eines Landes - abhängen, sind bestimmte Mindestbedingungen vonnöten, so etwa sauberes Trinkwasser und Sanitäranlagen, Kliniken und andere Gesundheitseinrichtungen, geschultes und angemessen entlohntes Personal sowie eine Grundausstattung an unentbehrlichen Arzneimitteln

23 Vgl. etwa Schmidhuber (2013) und (2014).

24 Vgl. auch den Beitrag von Bielefeldt in diesem Band.

25 CESCR E/C.12/2000/4, 11. August 2000, Ziff. 11.

26 Der Begriff der palliativen Gesundheitsversorgung findet sich noch nicht im UN-Sozialpakt, ist aber als Bestandteil einer umfassenden, auch lindernden medizinischen Behandlung anzusehen. Wohl aber im Allgemeinen Kommentar $\mathrm{Nr}$. 14, CESCR E/C.12/2000/4, 11. August 2000, Ziff. 34.

27 Ebd., Ziff. 12. 
gemäß WHO-Standards. Jedem Menschen muss zudem der Zugang zu medizinischen Einrichtungen und Behandlungen offen stehen - und zwar in mehrfacher Hinsicht:

- diskriminierungsfrei, auch und gerade im Falle besonders schutzbedürftiger und marginalisierter Gruppen der Bevölkerung;

- physisch, d.h. in sicherer Reichweite und zugänglich gerade auch für Frauen, Kinder, ältere Menschen sowie für Menschen mit chronischen Krankheiten oder Behinderungen;

- wirtschaftlich - dergestalt, dass öffentliche oder private medizinische Einrichtungen und Behandlungen für alle, auch arme und sozial benachteiligte Menschen, bezahlbar und erschwinglich sind;

- informiert - in dem Sinne, dass die Menschen das Recht haben, gesundheitsrelevante Informationen zu suchen, zu erhalten und weiterzugeben, sofern dabei nicht der persönliche Vertrauensschutz beeinträchtigt wird.

Im Sinne der Annehmbarkeit müssen die medizinischen Einrichtungen und die ärztliche Betreuung nach Grundsätzen medizinischer Ethik erfolgen, Vertraulichkeit gewähren und auf die Verbesserung des Gesundheitszustands der Betroffenen abzielen. Dabei sind die kulturellen Prägungen der einzelnen Menschen, aber auch von Minderheiten und Gemeinschaften, zu respektieren sowie geschlechts- und altersbedingte Besonderheiten gezielt zu berücksichtigen. Gefordert ist weiterhin, dass medizinische Einrichtungen und die ärztlichen Behandlungen unter wissenschaftlichen und medizinischen Gesichtspunkten geeignet und von angemessener Qualität sind. Die medizinische Versorgung hat durch geschultes Personal zu erfolgen und muss medizinischen Standards für Arzneimittel, Geräte, Ausstattungen und Hygiene genügen.

\section{Die Staaten in der Pflicht}

Die Hauptverantwortung für die Umsetzung der Menschenrechte tragen, völkerrechtlich gesehen, die Staaten. In Form internationaler Menschenrechtsabkommen verpflichten sich die Staaten gegenseitig dazu, die Men- 
schenrechte der Einzelpersonen unter ihrer Hoheitsgewalt ${ }^{28} \mathrm{zu}$ achten, zu schützen und $\mathrm{zu}$ gewährleisten. Aus völkerrechtlicher $\operatorname{Sicht}^{29}$ binden die Menschenrechte dabei alle staatlichen Gewalten, Organe und Institutionen, und zwar gleich, ob diese auf Bundes-, Landes- oder Kommunalebene angesiedelt sind oder ob es sich um über- oder untergeordnete Behörden handelt. Die staatliche Verpflichtung erstreckt sich auch auf solche privaten Akteure, denen die Erfüllung öffentlicher Aufgaben übertragen wird oder die im Auftrag, unter Anleitung oder unter Kontrolle des Staates agieren. ${ }^{30}$ Jüngeren völkerrechtsdogmatischen Entwicklungen zufolge begründen die Menschenrechte dabei staatliche Achtungs-, Schutz- und Gewährleistungspflichten.

\subsection{Staatliche Achtungspflichten}

Achtungspflichten (obligations to respect) bilden den Kern einer liberalen Menschenrechtstheorie, die den Schutz der Freiheitssphäre des einzelnen Menschen gegenüber Eingriffen des Staates und damit den Abwehrcharakter der Menschenrechte in den Mittelpunkt stellt. Sie verpflichten die Staaten, den einzelnen Menschen nicht direkt oder indirekt an der Ausübung seiner Menschenrechte zu hindern - und dort, wo sie es tun, entsprechende Eingriffe zu beheben. Es handelt sich also vor allem um Unterlassungspflichten, ggf. verbunden mit restitutorischen Beseitigungspflichten.

Im Hinblick auf unser Thema fordern die Achtungspflichten von den Staaten, das Recht auf Gesundheit der Menschen nicht selbst zu verletzen. Die Staaten dürfen also keine Handlungen vornehmen, die dem Recht auf Gesundheit zuwiderlaufen und »die zu körperlichen Schäden, unnötigen Erkrankungen und vermeidbarer Sterblichkeit führen können «. ${ }^{31}$ Worin könnten solche Handlungen bestehen? In Bezug auf das Gesundheitswesen fallen darunter prinzipiell alle staatlichen Handlungen, welche die Verfügbarkeit, die Zugänglichkeit oder die Angemessenheit und Qualität der

28 Auf extraterritoriale Pflichten gehe ich später kurz ein.

29 Über die Frage, wie die Verantwortlichkeiten, Zuständigkeiten und Kompetenzen im nationalen Recht verteilt sind, ist damit nichts gesagt.

30 Vgl. Kälin/Künzli (2008), 92.

31 CESCR E/C.12/2000/4, 11. August 2000, Ziff. 50. 
Gesundheitsversorgung derart beeinträchtigen, dass die Gesundheit der Menschen gefährdet oder geschädigt wird.

Der UN-Ausschuss führt in seinem Allgemeinen Kommentar als zentrales Beispiel die Verweigerung der medizinischen Versorgung an und stellt damit auf einen diskriminierungsfreien, offenen Zugang zur Gesundheitsversorgung ab. Hier ist $\mathrm{zu}$ prüfen, ob Gesetze, Verordnungen oder auch nur die Praxis in öffentlichen Gesundheitseinrichtungen bestimmten Bevölkerungsgruppen oder Personen einen offenen Zugang erschweren oder verwehren. Das können, je nach Kontext, beispielsweise ethnische Gruppen oder nationale Minderheiten, Menschen mit Behinderungen, psychisch Kranke oder Frauen sein, oder auch etwa ausländische Staatsangehörige Flüchtlinge, »irreguläre« Migranten oder Gefängnisinsassen. Problematisch gestaltet sich mitunter auch die Gesundheitsversorgung von Lesben, Schwulen, Bisexuellen, Trans* und Inter* (kurz: LGBTI-Personen) zumal wenn diese, wie es in etlichen Ländern der Fall ist, eine strafrechtliche Verfolgung befürchten müssen.

Aber auch die Art und Weise, wie Menschen in staatlichen Gesundheitseinrichtungen behandelt werden, ist mit Blick auf die Achtungspflichten relevant. Nicht nur verwehrte, sondern auch unzureichende, fehlerhafte oder missbräuchliche Behandlungen können Eingriffe in das Recht auf Gesundheit darstellen. Hier geht es also um die Angemessenheit und Qualität der Gesundheitsversorgung. $\mathrm{Zu}$ prüfen ist etwa, ob die verfügbaren Behandlungsmöglichkeiten ausgeschöpft und medizinische Standards eingehalten werden. Dies ist beileibe nicht immer der Fall. ${ }^{32}$ Zugleich gibt es zur Genüge historische und aktuelle Beispiele für gezielte gesundheitsschädliche Handlungen in staatlichen Gesundheitseinrichtungen: Sie reichen von medizinischen Versuchen mit Patienten ohne deren Einwilligung ${ }^{33}$ über die Misshandlungen psychisch kranker Menschen in staatlichen »Verwahranstalten $\ll^{34}$ (oder auch, wie etwa in China, des Missbrauchs der Psychiatrie

32 Vgl. etwa die Diskussion um Fehler und Ethik in der Medizin, z.B. in Frewer et al. (2013).

33 Bedeutsam sind in diesem Zusammenhang auch die grenzüberschreitenden Versuche westlicher Pharmafirmen in der DDR, vgl. Erices et al. (2015).

34 Vgl. etwa den Bericht des damaligen Sonderberichterstatters zum Recht auf Gesundheit mit dem Schwerpunkt »mental disability«, Paul Hunt: E/CN.4/2005/41, 11. Februar 2005, Ziff. 9. 
als Verwahrungsort für »uneinsichtige« politische Dissidenten) bis zur Zwangssterilisation von Menschen mit Behinderungen ${ }^{35}$ oder von Frauen. In Peru wurden beispielsweise während der Amtszeit Alberto Fujimoris (1990-2000) im Rahmen der Geburtenkontrolle rund 300.000 Frauen und etwa 22.000 Männer, vor allem Indigene und arme Bäuerinnen und Bauern, ohne vorherige Aufklärung und Einwilligung zwangssterilisiert. ${ }^{36}$ Eine strafrechtliche Verfolgung, die von den Betroffenen gefordert wird, steht bis heute weitestgehend aus. ${ }^{37}$

Im Einzelnen werfen die staatlichen Achtungspflichten im Gesundheitswesen viele Fragen auf. Als nur ein Beispiel sei die bereits angesprochene Diskriminierungsproblematik genannt. Abgesehen davon, dass die medizinische Behandlung individuell auf die jeweiligen Patienten zugeschnitten werden muss, stellt sich die Frage, auf welche Gesundheitsleistungen sich der menschenrechtliche Anspruch auf diskriminierungsfreie Gleichbehandlung bezieht. Sicherlich nicht nur auf eine Minimalversorgung! Wie sieht es aber etwa mit unserem Asylbewerberleistungsgesetz (AsylbLG) aus? Solange Flüchtlinge, Asylsuchende und Geduldete Gesundheitsleistungen auf Grundlage des Asylbewerberleistungsgesetzes erhalten, ${ }^{38}$ sind die entsprechenden Leistungsansprüche auf die Versorgung bei akuten Erkrankungen und Schmerzen, auf die ärztliche und pflegerische Betreuung von Müttern und Wöchnerinnen sowie auf amtlich empfohlene Schutzimpfungen und medizinisch gebotene Vorsorgeuntersuchungen beschränkt (AsylbLG § 4). Ausgeschlossen ist damit - zumindest im Grundsatz - die Behandlung chronischer Erkrankungen, soweit sie nicht mit Schmerzzuständen verbunden sind, ${ }^{39}$ oft mit schwerwiegenden Folgen für die Betroffenen. Ebenso ist die zahnärztliche Versorgung völlig ungenü-

35 Vgl. etwa: www.enil.eu/news/sterilization-of-women-and-girls-with-disabilitiesa-briefing-paper-november-2011/ [01.08.2015].

36 Siehe hierzu Jaichand/O'Donnell (2010). Eine entsprechende Dokumentation »Zwangssterilisation in Peru«strahlte ARTE am 4. Juni 2010 aus.

37 Vgl. etwa die Presseberichte von Anliker (2014) und Cordier (2015).

38 Bis zu der am 1. März 2015 in Kraft getretenen Gesetzesreform galt dies für einen Zeitraum von 48 Monaten nach Einreise. Mit der Gesetzesreform wurde der Zeitraum auf 15 Monate Aufenthalt im Bundesgebiet (ohne wesentliche Unterbrechungen) verkürzt.

39 Vgl. Kaltenborn (2015). 
gend. ${ }^{40}$ Auch gestaltete sich - schon vor dem massiven Anstieg der Flüchtlingszahlen im Jahr 2015 - die psychosoziale Versorgung von Flüchtlingen als problematisch. ${ }^{41}$

Selbst angesichts legitimer ordnungspolitischer Interessen des Staates ist die legale und faktische Ungleichbehandlung im Gesundheitswesen von Menschen, abhängig von Staatsangehörigkeit oder Aufenthaltsstatus, aus menschenrechtlicher Sicht kritisch zu bewerten, zumal, wenn die resultierende Gesundheitsversorgung offenkundig unzureichend ist und selbst eine Notversorgung durch bürokratische Hindernisse mitunter behindert wird. ${ }^{42}$ Dies gilt auch in Bezug auf Migrantinnen und Migranten, die sich »irregulär« im Land aufhalten. Diese haben zwar grundsätzlich das Recht auf Zugang zur Gesundheitsversorgung, in Deutschland etwa auf Grundlage des Asylbewerberleistungsgesetzes. Trotz aller nationalen Besonderheiten und vereinzelter Positivbeispiele ist jedoch mit Heinz-Jochen Zenker festzustellen: »Überall stehen Menschen ohne Papiere am unteren Ende des $\mathrm{Zu}$ gangs zu einer bedarfsgerechten Medizin, was Menschenrechtskonventionen und der Europäischen Sozialcharta widerspricht. ${ }^{43}$ Hinzu kommt, dass die jeweiligen Aufspür-, Melde- und Festnahmepraktiken viele Menschen »ohne Papiere« de facto oft davon abhalten, Gesundheitsleistungen in Anspruch zu nehmen. ${ }^{44}$

Die Gesundheit gefährdende und schädliche staatliche Handlungen können zudem jene Parameter betreffen, welche außerhalb des Gesundheitswesens die Gesundheit der Menschen beeinflussen. Wie bereits dargelegt wurde, nennt hier der UN-Ausschuss für WSK-Rechte ausdrücklich den Zugang zu sauberem und gesundem Trinkwasser, eine angemessene Sanitärversorgung, unbedenkliche Nahrung und Unterkunft, gesunde Arbeits- und Umweltbedingungen sowie gesundheitsbezogene Informationen. Vor diesem Hintergrund ist - etwa in Gestalt menschenrechtlicher Folgen-

40 Vgl. Lindner (2015), 81.

41 Vgl. BAfF (2015).

42 Vgl. Lindner (2015) sowie Misbach (2015). Die Bundesregierung sieht hingegen die Gesundheitsversorgung der Leistungsberechtigten als angemessen an; vgl. Bundestagsdrucksache 18/4758, 27. April 2015. Aufschlussreich ist auch die Plenardebatte im Bundestag, 115. Sitzung, 2. Juli 2015, 11078-11093.

43 Zenker (2011), 96.

44 Vgl. etwa Fundamental Rights Agency (2012) sowie Mylius/Frewer (2015). 
abschätzungen - zu prüfen, inwieweit von staatlichen Maßnahmen in den verschiedenen Politikbereichen (Wirtschafts-, Energie-, Verteidigungspolitik etc.) Gesundheitsgefahren und -schäden ausgehen. Auch können staatliche Betriebe oder öffentliche Infrastrukturmaßnahmen das Recht auf Gesundheit verletzen, wenn der Gesundheitsschutz am Arbeitsplatz vernachlässigt oder wenn die Umwelt kontaminiert wird. Eine Mitverantwortung ergibt sich ggf. auch durch die Kooperation mit privaten Unternehmen. Eine landmark decision traf diesbezüglich die Afrikanische Menschenrechtskommission gegen die damalige Militärregierung Nigerias. Im Rahmen der nationalen Ölförderung hatte diese gemeinsam mit einem großen Ölkonzern erhebliche Umwelt- und Gesundheitsschäden im NigerDelta verursacht. Die Kommission kam zu dem Schluss, dass die in der Banjul-Charta garantierten Rechte auf Gesundheit und auf eine angemessene - hier: gesunde - Umwelt verletzt wurden. ${ }^{45}$

Auch die Zurückhaltung oder Falschdarstellung gesundheitsrelevanter Informationen kann eine Verletzung staatlicher Achtungspflichten darstellen. So darf der Staat (lebens-)wichtige Gesundheitsinformationen etwa im Hinblick auf Ansteckungsschutz, Seuchenprävention oder auch Umweltkatastrophen weder zurückhalten noch verfälschen. Aus der Perspektive des Rechts auf Gesundheit wurde beispielsweise kritisiert, dass die Regierung Zimbabwes den Ausbruch einer Cholera-Epidemie im Jahre 2008 lange Zeit verschwieg und diese zu früh für beendet erklärte. ${ }^{46}$ Auf berechtigte menschenrechtliche Kritik stieß auch der Umstand, dass die südafrikanische Regierung unter Staatspräsident Thabo Mbeki (1999-2008) die Ansteckungsgefahr mit HIV bagatellisierte und sogar den Zusammenhang zwischen HIV und AIDS zeitweise negierte. ${ }^{47}$ Umgekehrt können Maßnahmen zur Eindämmung von Epidemien, wie etwa Zwangsquarantänen im Falle von Ebola-Patienten in Sierra Leone, Guinea und Liberia im Jahre 2014, zu Eingriffen in die Freiheits- und Beteiligungsrechte der Betroffenen führen. Inwieweit solche Eingriffe gerechtfertigt sind, lässt sich beispielsweise an-

45 Communication Nr. 155/2001, SERAC and CESR v Nigeria (2001), 15th Annual Activity Report of ACHPR: 2000-2002. Der Fall ist auch in der Sekundärliteratur gut dokumentiert, siehe etwa Keetharuth (2009) und Nolan (2009).

46 Vgl. Zimbabwe Human Rights NGO Forum (2009).

47 Siehe in diesem Zusammenhang die Maßnahmen der »Treatment Action Campaign«; vgl. Heywood (2009). 
hand der »Siracusa Principles « diskutieren, welche Einschränkungen (limitations) oder die Aussetzung (derogation) bürgerlich-politischer Rechte im Falle entsprechender public emergencies behandeln. ${ }^{48}$

\subsection{Staatliche Schutzpflichten}

Schutzpflichten (obligations to protect) bestehen in der staatlichen Verpflichtung, den einzelnen Menschen gegen tatsächliche oder drohende Eingriffe in seine Menschenrechte durch Dritte, für gewöhnlich private Akteure, zu schützen. Bei Schutzpflichten handelt es sich nicht um Handlungsverbote, sondern um Handlungsgebote für den Staat. Welche Schutzmaßnahmen der Staat ergreift, liegt, soweit diese zielführend und menschenrechtskonform ausgestaltet sind, grundsätzlich im Ermessen der staatlichen Entscheidungsträger, die diesbezüglich über einen weiten Ermessens- und Gestaltungsspielraum verfügen. Daher ist es nicht immer leicht, etwaige Verletzungen staatlicher Schutzpflichten festzustellen. Sie können sich beispielsweise durch staatliches Unterlassen ergeben, wenn

- öffentliche Stellen Kenntnis von einer aktuellen oder drohenden Gefährdung haben oder bei erforderlicher Sorgfalt haben können,

- sie trotz entsprechender Kenntnis nicht im Rahmen ihrer verfügbaren Mittel geeignete Schutzmaßnahmen ergreifen und

- doch zugleich menschenrechtskonforme Gegenmaßnahmen möglich wären. ${ }^{49}$

Im Gesundheitswesen können solche Verletzungen beispielsweise dann auftreten, wenn der Staat es zulässt, dass private Gesundheitseinrichtungen gegen medizinische Standards verstoßen, oder wenn er nichts dagegen unternimmt, dass schädliche oder unwirksame Medikamente im Umlauf sind (wie dies in nicht wenigen Ländern der Fall ist). Im Sinne der Schutzpflichten muss der Staat private Gesundheitseinrichtungen, -leistungen und -produkte hinreichend regulieren und kontrollieren, um sicherzustellen, dass die Menschen tatsächlich medizinische Hilfe erfahren und nicht in ihrer Gesundheit geschädigt werden. Dabei kann es prinzipiell zu denselben

48 E/CN.4/1985/4, Annex (1985).

49 Vgl. Kälin/Künzli (2008), 126. 
Missständen kommen wie in staatlichen Gesundheitseinrichtungen (siehe oben), nur dass hier die Schädigungen von privaten Akteuren ausgehen. Besonders augenfällig wird die staatliche Schutzpflicht, wenn es beispielsweise in privaten Gesundheitseinrichtungen zu vermeidbaren Todesfällen kommt und zugleich der Staat seiner Regulierungs- und Kontrollpflicht nicht nachgekommen ist. Diesbezüglich gibt es eine Reihe einschlägiger Entscheidungen, beispielsweise des Interamerikanischen Gerichtshofs für Menschenrechte ${ }^{50}$ oder auch im Rahmen des Beschwerdeverfahrens zu CEDAW. $^{51}$

Menschenrechtlich von großer Relevanz ist auch die Frage, wie offen der Zugang zu privaten Gesundheitsleistungen sein muss. Zwar ist es wenig umstritten, dass auch private Gesundheitseinrichtungen keinem Menschen eine Notversorgung verwehren dürfen, doch der Zugang zu darüber hinaus gehenden privaten Leistungen ist gemeinhin nicht für jedermann möglich oder erschwinglich. Dies ist insbesondere dann ein Problem, wenn gleichzeitig der öffentliche Gesundheitssektor nicht flächendeckend verfügbar oder von schlechter Qualität ist. In diesem Fall muss der Staat dafür Sorge tragen, dass eine qualitativ angemessene Gesundheitsversorgung allen Menschen offen steht - sei es durch eine entsprechende Regulierung privater Anbieter oder sei es durch den Ausbau des öffentlichen Gesundheitssektors, was bereits auf die (später behandelten) Gewährleistungspflichten verweist.

Ebenso wie die Achtungspflichten erstrecken sich die Schutzpflichten nicht nur auf die Gesundheitsversorgung im engeren Sinne, sondern umfassen auch die Arbeits- und Lebensbedingungen, welche die Gesundheit der Menschen mitbestimmen. Hierbei beziehen sich die Schutzpflichten zum einen auf eine angemessene Regulierung und Kontrolle des Gesundheitsschutzes bei der Arbeit, zum anderen betreffen sie den - für die Gesundheit der Menschen unentbehrlichen - Schutz einer intakten und gesunden Umwelt vor privat(wirtschaftlich)en Eingriffen. Weltweit sind jedoch gesundheitsschädigende Arbeitsbedingungen und Umweltverschmutzungen durch private Unternehmen dokumentiert - sei es beim Abbau natürlicher Ressourcen, in der Landwirtschaft oder in der verarbeitenden Industrie. Man

50 Ximenes-Lopes v. Brasil, Series C 149, 2006; siehe auch Nolan (2009).

51 Alyne Silva Pimentel v. Brazil, CEDAW, Mitteilung Nr. 17/2008, Urteil vom 25. Juli 2011. 
denke hier nur an gesundheitsgefährdende Arbeitsbedingungen in der Textilbranche in Südasien, die mit den Bränden und Fabrikeinstürzen in Bangladesch und Pakistan auch hierzulande öffentliche Aufmerksamkeit erregten. ${ }^{52}$ Gerade in Entwicklungs- und Schwellenländern ist die Problematik offensichtlich. Erschwerend kommt hinzu, dass dort in großem Stil informelle Arbeitsverhältnisse vorherrschen.

Staatliche Schutzpflichten zur Vermeidung von Berufsunfällen, Berufskrankheiten und Umweltverschmutzungen ergeben sich nicht nur aus Art. 12 Abs. 2 (b) des UN-Sozialpakts, sondern auch aus Konventionen der International Labour Organization (ILO). Im Rahmen des Europarates kann ggf. auch eine Verletzung der Europäischen Sozialcharta geltend gemacht werden. Bei Kollektivbeschwerden gegen Griechenland sah beispielsweise der Europäische Sozialausschuss das Recht auf Gesundheit verletzt, da die nationalen Autoritäten - bei allen zugestandenen Ermessensund Handlungsspielräumen - zu wenig unternommen hätten, um die Gesundheit von Anwohnern vor Wasser- und Luftverschmutzungen durch private Unternehmen zu schützen. ${ }^{53}$ Eine eigene Problematik stellt die oft privatisierte Müllentsorgung dar. ${ }^{54}$

Bedeutsam ist weiterhin der Gesundheitsschutz von Verbrauchern. So muss der Staat verhindern, dass hochgradig belastete und gesundheitsschädliche Verbrauchsgüter, allen voran Lebensmittel, vertrieben werden. Zugleich wurden in den vergangenen Jahren auch Rauchverbote und Forderungen nach Maßnahmen gegen den Alkohol- und Drogenkonsum mit dem Recht auf Gesundheit begründet. Noch weiter gehen Forderungen nach

52 Hintergrundinformationen zu den Arbeitsbedingungen in der Textilindustrie finden sich auf den Homepages u.a. von »Clean Clothes Campaign« (www.cleanclothes.org), »Christliche Initiative Romero« (www.ci-romero.de), ECCHR (www.ecchr.eu), »medico international« (www.medico.de) sowie der »Fair Wear Foundation« (www.fairwear.org); siehe auch Burckhardt (2014).

53 Siehe die Urteile zu den Beschwerden Nr. 30/2005 und Nr. 72/2011.

54 In den süditalienischen Provinzen Neapel und Caserta führte beispielsweise die kriminelle, illegale Entsorgung von Sonder- und Giftmüll nachweislich zu gesundheitlichen Schäden der Bevölkerung. Vgl. Camera dei deputati: D.L. 136/2013: Emergenze ambientali e industriali, 6. Februar 2014; Emergenze rifiuti, 21.11.2014; abrufbar unter: www.camera.it [01.06.2015]. 
Schutz vor Übergewicht ${ }^{55}$ oder nach einer »Global Convention to Protect and Promote Healthy Diets «. Die letztgenannte Forderung wurde vom damaligen UN-Sonderberichterstatter zum Recht auf Nahrung, Olivier de Schutter, erhoben und damit begründet, dass ungesunde Ernährung eine größere Gefahr für die Gesundheit darstelle als Tabak. ${ }^{56}$ Bislang werden aber in puncto Essgewohnheiten und gesunde Ernährung noch kaum staatliche Pflichten aus dem Recht auf Gesundheit abgeleitet. Hier bedürfte es sicherlich einer eingehenden Diskussion darüber, ob und inwieweit über Informations- und Aufklärungspflichten hinaus in die Freiräume und den Verantwortungsbereich der einzelnen Menschen eingegriffen werden kann und soll.

Anders stellt sich dies bei physischen Gewalttaten dar. Hier nimmt der UN-Ausschuss für WSK-Rechte - und zwar auf Grundlage des Rechts auf Gesundheit - die Staaten in die Pflicht, private Gewalt, gerade auch die häusliche, zu bekämpfen und die Straftäter zu verfolgen. ${ }^{57}$ So betont er immer wieder die Verpflichtung, Frauen und Mädchen vor sexueller oder anderer Gewalt zu schützen, und zwar in ganz unterschiedlichen Gefährdungslagen und auch bei der Nutzung anderer Menschenrechte: auf dem Schulweg, auf dem Weg zur Arbeit oder am Arbeitsplatz, beim Aufsuchen von Wasserstellen oder sanitären Anlagen oder auch in Notlagern und Flüchtlingscamps. Zudem sei der Staat verpflichtet, gesundheitsschädliche kulturelle Praktiken zu unterbinden, allen voran die Praxis der weiblichen Genitalverstümmelung (Female Genital Mutilation, kurz: FGM). Sowohl der UN-Ausschuss für WSK-Rechte als auch der CEDAW-Ausschuss haben weibliche Genitalverstümmelung immer wieder als Menschenrechtsverletzung ausgewiesen, wenngleich nicht immer auf Grundlage des Rechts auf Gesundheit. ${ }^{58}$

55 So etwa die Forderung des UN-Ausschusses für WSK-Rechte gegenüber Schweden: E/C.12/SWE/CO/5, 18. November 2008, Ziff. 23.

56 So am 19. Mai 2014 in Genf; vgl. auch seinen Bericht zu diesem Thema: A/HRC/19/59, 26.12.2011.

57 Vgl. CESCR E/C.12/2000/4, 11. August 2000, Ziff. 51.

58 Vgl. hier etwa die »Abschließenden Bemerkungen« zum Tschad: E/C.12/ TCD/CO/3, 16. Dezember 2009, Ziff. 19; CEDAW/C/TCD/CO/1-4, 21. Oktober 2011, Ziff. 22-23. Siehe generell zur FGM auch Graf (2013). 


\subsection{Staatliche Gewährleistungspflichten}

Gewährleistungspflichten (obligations to fulfill) stellen »Leistungsrechte « im engeren Sinne dar. Sie verpflichten die Staaten, die möglichst umfassende Ausübung der Menschenrechte durch aktives staatliches Handeln zu ermöglichen. Hier geht es also darum, über entsprechende Gesetze, Einrichtungen und Verfahren sowie über staatliche Leistungen in Form von Geld, Gütern oder Dienstleistungen die Voraussetzungen für die Umsetzung des Rechts auf Gesundheit zu schaffen.

Wie eingangs des Beitrags aufgezeigt wurde, sehen bereits die jeweiligen Menschenrechtsabkommen vielfältige Schritte zur Umsetzung des Rechts auf Gesundheit vor. ${ }^{59}$ Dementsprechend weit sind die Gewährleistungspflichten im Bereich der Gesundheit gefächert. Sie umfassen zunächst den Aufbau und Unterhalt medizinischer und gesundheitsrelevanter Infrastrukturen. So müssen die Staaten dafür Sorge tragen, dass notwendige medizinische Einrichtungen, Leistungen und Programme mit geschultem Personal zur Verfügung stehen, für jedermann zugänglich sind und die Menschen zugleich Zugang zu angemessener Nahrung, Unterkunft, Sanitäranlagen, Trinkwasser sowie zu unentbehrlichen Arzneimitteln haben. Weiterhin sehen die Menschenrechtsabkommen konkrete Schritte der Staaten vor, um die Gesundheitslage der Bevölkerung im Allgemeinen sowie einzelner, besonders bedürftiger und vulnerabler Gruppen zu verbessern, etwa von Kindern, Müttern, alten Menschen oder Menschen mit Behinderungen. Hierbei reicht es für gewöhnlich nicht nur, den Zugang zur medizinischen Versorgung sicherzustellen. Oft sind auch die sozioökonomischen und soziokulturellen Bedingungen zu verändern, welche die Gesundheitslage mitbestimmen, etwa Armut oder gesellschaftliche Marginalisierung und Ausgrenzung.

Die Ausgestaltung der Gesundheitssysteme - ob öffentlich und/oder privat - sowie konkreter gesundheitspolitischer Maßnahmen liegt grund-

59 Es sei an dieser Stelle darauf verzichtet, die vielfältigen menschenrechtsbezogenen Maßnahmen im Gesundheitsbereich einzeln darzulegen. Siehe hierzu die Berichte und Empfehlungen der charta- und vertragsgestützten UN-Menschenrechtsorgane, die sich auf der Homepage: www.ohchr.org befinden. Ebenso lohnend ist die Lektüre der WHO-Dokumente, die sich auf das Menschenrecht auf Gesundheit beziehen: www.who.int/topics/human_rights/en/ [01.10.2015]. 
sätzlich im Ermessen der jeweiligen Staaten, zumindest, solange diese menschenrechtliche Prinzipien (wie Transparenz, Partizipation, Nicht-Diskriminierung) beachten und die allgemeine Verfügbarkeit, Zugänglichkeit, Annehmbarkeit und Qualität der Gesundheitsversorgung gewährleisten. ${ }^{60}$ Privatisierte oder vertraglich ausgelagerte Gesundheitsleistungen entheben die Staaten allerdings nicht ihrer Pflicht, dies sicherzustellen. So müssen die Regierungen beispielsweise verhindern, dass eine qualitativ angemessene Gesundheitsversorgung nur zahlungskräftigen Patienten vorbehalten bleibt. Allein 100 Millionen Menschen werden der WHO zufolge jedoch jährlich in die Armut getrieben, weil sie für Gesundheitsleistungen selbst zahlen müssen (out-of-pocket payments). ${ }^{61}$ Dies verweist nicht nur auf den engen Zusammenhang zwischen dem Recht auf Gesundheit und dem Recht auf soziale Sicherheit, ${ }^{62}$ sondern häufig auch auf das Problem einer miserablen öffentlichen Gesundheitsversorgung. Aus diesem Grund, so wird etwa aus Indien berichtet, ziehen mitunter selbst arme Menschen kostenpflichtige private Gesundheitsleistungen der unentgeltlichen Behandlung in staatlichen Krankenhäusern vor - und verschulden sich dabei oft haushoch. $^{63}$

Da die Umsetzung der Gewährleistungspflichten im Falle des Rechts auf Gesundheit mit hohen Kosten verbunden ist, stoßen viele Länder bei der Umsetzung dieses Rechts an ihre Grenzen. Gerade viele Entwicklungsländer haben immense Schwierigkeiten, eine flächendeckende medizinische Versorgung sicherzustellen und die teils gravierenden Mängel im Gesundheitswesen zu überwinden. Geschweige denn können sie sich eine so umfassende und teure Gesundheitsversorgung leisten wie die reichen Wohlstandsgesellschaften. Das nimmt aber Entwicklungsländer nicht aus der Pflicht, auf Grundlage ihrer verfügbaren Ressourcen ${ }^{64}$ Maßnahmen zu ergreifen, um das Recht auf Gesundheit schrittweise umzusetzen.

60 Vgl. etwa Hunt/Backman (2013).

61 Vgl. www.who.int/factfiles/universal_health_coverage/en/index.html [01.10. 2015]. Siehe auch den Beitrag von Heinicke et al. im vorliegenden Band.

62 Vgl. Krennerich (2014).

63 Vgl. Shankar/Mehta (2008), 155.

64 Die deutsche Übersetzung von »Ressourcen« legt sinnvollerweise einen umfassenden Ressourcenbegriff zugrunde, der sich nicht nur auf finanzielle Ressour- 
Die Verpflichtung einer nur schrittweisen, progressiven Rechtsumsetzung, wie sie der UN-Sozialpakt vorsieht (Art. 2 Abs. 1), trägt der Tatsache Rechnung, dass sich - angesichts schwer überwindbarer sozialer Missstände und knapper Ressourcen - die sozialen Menschenrechte nicht von heute auf morgen umsetzen lassen, insbesondere nicht jene Rechtskomponenten, deren Umsetzung umfangreiche staatliche Leistungen und langfristiges Handeln erfordern. Im Hinblick auf das Recht auf Gesundheit (und anderer WSK-Rechte) trifft dies gerade auf die Gewährleistungspflichten $\mathrm{zu}$.

Jedoch kann die Verpflichtung zu einer progressiven Umsetzung nicht als Entschuldigung dienen, untätig zu bleiben. Vielmehr hat der Staat die prozedurale Verpflichtung, konkrete und zielführende Politiken zu entwerfen und Maßnahmen durchzuführen, die im Ergebnis auf die Umsetzung des Rechts auf Gesundheit abzielen. So sind die Staaten verpflichtet, in einem partizipatorischen und transparenten Prozess umgehend eine umfassende nationale Gesundheitsstrategie auszuarbeiten, um die bestehenden (und $\mathrm{zu}$ identifizierenden) Gesundheitsprobleme in dem jeweiligen Land anzugehen.

Zudem müssen einige Kernverpflichtungen, soweit möglich, unmittelbar umgesetzt werden. ${ }^{65}$ Dazu zählt der UN-Ausschuss für WSK-Rechte immerhin den diskriminierungsfreien Zugang zu medizinischen Einrichtungen und ärztlicher Betreuung sowie den Zugang zu einem Mindestmaß an grundlegenden Nahrungsmitteln, zu Unterkunft, Sanitäranlagen und sicherem Trinkwasser sowie die Versorgung mit elementaren Arzneimitteln. Hinzu kommt eine gerechte Verteilung von medizinischen Einrichtungen und ärztlicher Betreuung, wobei hier insbesondere das Problem der Unterversorgung ländlicher Gebiete im Blickpunkt steht. ${ }^{66}$ Von vergleichbarer Priorität sieht der Ausschuss folgende Aspekte an: die Gesundheitsfürsorge im Hinblick auf die reproduktive Gesundheit, Mutterschaft und von Kindern; Impfungen gegen verbreitete Infektionskrankheiten; Maßnahmen zur Verhinderung, Behandlung und Bekämpfung epidemischer und endemischer Krankheiten; Aufklärung und Informationen über die wesentlichen

cen beschränkt, sondern alle Arten von Ressourcen umfasst; vgl. auch Klee (2000), 122-129 und Engbruch (2008), 108.

65 Vgl. den Beitrag von Müller in diesem Band.

66 CESCR E/C.12/2000/4, 11. August 2000, Ziff. 43. 
Gesundheitsprogramme der Gemeinschaft sowie eine angemessene Ausbildung des Personals im Gesundheitswesen. ${ }^{67}$

Darauf aufbauend sind Maßnahmen zu ergreifen, die das Recht auf Gesundheit fortschreitend und umfänglich verwirklichen. Die - mit gehörigem Fortschrittsoptimismus seinerzeit verankerte - Verpflichtung einer progressiven Umsetzung geht zwar nicht mit einem absoluten Regressionsverbot einher. Unvermeidbare Rückschritte sind jedoch erklärungsbedürftig. Von entscheidender Bedeutung ist dabei letztlich, dass der Staat seine verfügbaren Ressourcen - und damit auch einen angemessenen Anteil des Staatsanteils und etwaiger internationaler Hilfen - auch wirklich für die Umsetzung des Rechts auf Gesundheit einsetzt. Ein Staat, der hierzu nicht willens ist, verletzt aus Sicht des UN-Ausschusses für WSK-Rechte seine Verpflichtungen gemäß Art. 12 des UN-Sozialpaktes. ${ }^{68}$

Dabei ist freilich immer zu klären, wie viel Ressourcen angemessen sind. Der Wortlaut des UN-Sozialpaktes, der den jeweiligen Staat verpflichtet, das »maximum of its available ressources« auszuschöpfen, ist wenig hilfreich. Es liegt auf der Hand, dass ein Staat nicht all seine Ressourcen für die Umsetzung einzelner Menschenrechte ausgeben kann, so wichtig diese auch sein mögen. Die Verpflichtung, alle Ressourcen auszuschöpfen, erfolgt also immer unter der Prämisse, dass dem Staat für die Erfüllung der verschiedenen Staatsaufgaben (und auch für die Umsetzung anderer Menschenrechte) hinreichend Mittel verbleiben. Dementsprechend geht es vor allem um die Gewichtung der Ziele wie auch um die Verteilung und Nutzung der verfügbaren Ressourcen. ${ }^{69}$

Nun stellt man in der Praxis rasch fest, dass die politische Prioritätensetzung und die Ausgabenpolitik vieler Staaten dringend menschenrechtlicher Korrekturen bedürfen. Salopp ausgedrückt: Wenn man sieht, wofür die Staaten Geld ausgeben, während gleichzeitig Abermillionen Menschen an vermeidbaren und heilbaren Krankheiten sterben, wird man unschwer feststellen, dass viele Ressourcen aus menschenrechtlicher Sicht falsch verteilt,

67 Ebd., Ziff. 44.

68 CESCR E/C.12/2000/4, 11. August 2000, Ziff. 47.

69 Dem Problem der »Prioritization of health interventions and respect of human rights « widmete sich u.a. der UN-Sonderberichterstatter zum Recht auf Nahrung in seinem Bericht an die UN-Generalversammlung vom 8. August 2007. UN Doc. A/62/214, Ziff. 11-32. 
nicht genutzt oder geschweige denn ausgeschöpft werden. In der Forderung, ein Höchstmaß an Ressourcen einzusetzen, liegt also viel kritisches Potenzial, denn es nimmt die Staaten in die Pflicht, angesichts anhaltender sozialer Notlagen mehr Ressourcen für die Umsetzung sozialer Menschenrechte zu mobilisieren. Bezeichnenderweise fordert der UN-Ausschuss für WSK-Rechte im Rahmen des Berichtsverfahrens zum UN-Sozialpakt die Staaten immer wieder auf, mehr Ressourcen für die Gesundheitsversorgung bereit zu stellen. Über ein nationales human rights budgeting und über systematische Ländervergleiche lassen sich die diesbezüglichen Diskussionen versachlichen, auch wenn hoher Mitteleinsatz allein noch keine angemessene Umsetzung des Menschenrechts auf Gesundheit garantiert.

Auf die Frage, ab wann das Menschenrecht auf Gesundheit vollumfänglich umgesetzt ist, gibt der UN-Ausschuss für WSK-Rechte keine abschließende Antwort. Die Forderung nach dem »erreichbaren Höchstmaß an Gesundheit « lässt viele Fragen offen. Da die Gesundheit der jeweiligen Menschen von vielfältigen kontingenten und damit beeinflussbaren Faktoren abhängt, lassen sich die Bedingungen für ein gesundes Leben prinzipiell immer weiter verbessern. Pragmatisch wird man daher die Umsetzung des Menschenrechts auf Gesundheit als einen fortwährenden Prozess betrachten müssen, der sich an den jeweiligen medizinischen und gesundheitlichen Standards orientiert, die auf nationaler bzw. internationaler Ebene festgelegt und weiterentwickelt werden. Offenkundig ist aber, dass sich das Menschenrecht auf Gesundheit nicht nur auf eine Grundversorgung beschränkt. Der Anspruch eines jeden Menschen auf das für ihn erreichbare Höchstmaß an Gesundheit dient daher als kritisches Korrektiv, damit eine umfassende medizinische Versorgung sowie gesunde Arbeits- und Lebensbedingungen nicht bestimmten Gruppen in der Gesellschaft vorenthalten bleiben.

\subsection{Die internationalen Dimensionen des Rechts auf Gesundheit}

Die Umsetzung des Rechts auf Gesundheit innerhalb der jeweiligen Staaten wird - im Positiven wie im Negativen - stark international beeinflusst. Einerseits gibt es vielfältige Bemühungen in der internationalen Gesundheitspolitik, der Entwicklungszusammenarbeit sowie im Rahmen der humanitären Hilfe, die Gesundheitsversorgung der Menschen gerade in Ent- 
wicklungsländern und in Krisenregionen zu verbessern. Der UN-Sozialpakt verpflichtet die Staaten sogar zu einer solchen Kooperation. Andererseits können internationale Politik und Rechtsvorschriften, etwa in Gestalt von Kreditauflagen oder Handels- und Patentregeln, auch die Umsetzung des Rechts auf Gesundheit beeinträchtigen. So weist der UN-Ausschuss für WSK-Rechte ausdrücklich auf die internationale Verpflichtung der Staaten hin, in ihrem bilateralen und multilateralen Handeln nicht das Recht auf Gesundheit in anderen Ländern zu beeinträchtigen. Auch beim Abschluss internationaler Abkommen und als Mitglieder internationaler Organisationen sind die Staaten daher verpflichtet, das Recht auf Gesundheit gebührend zu berücksichtigen.

Vielfach kritisiert wurden in der Vergangenheit beispielsweise patentrechtliche Vorgaben im Rahmen des sog. TRIPS-Abkommens der Welthandelsorganisation (World Trade Organisation, WTO). Holger Hestermeyer legte etwa dar, dass der Rechtsanspruch auf »access to medicine« Bestandteil der Menschenrechte auf Gesundheit und Leben ist; dass Patentrechtsregelungen, zu denen die WTO-Mitglieder verpflichtet sind, zu höheren Preisen für neue Medikamente führten; dass höhere Preise den Zugang zu Medikamenten für ärmere Bevölkerungsgruppen gerade in Entwicklungsländern erschwerten, mithin also einen Eingriff in das Menschenrecht darstellten; dass ein solcher Eingriff nicht hinreichend gerechtfertigt werden könne, auch nicht durch das Recht auf den Schutz der geistigen und materiellen Interessen von Urhebern wissenschaftlicher Werke (im Sinne des Art. 15 Abs. 1 des UN-Sozialpaktes), da dieses nicht die Patentrechte von Pharmaunternehmen schütze. ${ }^{70}$ Entsprechende flexible TRIPS-Regelungen in Form von Zwangslizenzen und Parallelimporten, um solche Konflikte zu lösen (»health safeguards «), werden de facto unterlaufen. ${ }^{71}$

Kritisch hinterfragen lassen sich aber auch manche Sparauflagen internationaler Kreditgeber. Denn Kürzungen im Sozial- und Gesundheits-

70 Vgl. Hestermeyer (2007). Bereits der UN-Ausschuss für WSK-Rechte hat in seinem Allgemeinen Kommentar Nr. 17 zum Art. 15 (1) c) des Sozialpaktes klargestellt, dass solche Patentrechte keine Menschenrechte sind und der Staat die Pflicht habe, die Menschen vor überhöhten Preisen für unentbehrliche Medikamente zu schützen; vgl. CESCR E/C.12/GC/17, 12. Januar 2006. Siehe auch Schneider (2006), 162-170.

71 Siehe etwa People's Health Movement et al. (2014), 288-299. 
bereich, wie sie die international erwirkten Austeritätsprogramme in den 1980er und 90er Jahren in Lateinamerika und anderen Weltregionen sowie in den vergangenen Jahren in Griechenland vorsahen, können hinsichtlich des Rechts auf Gesundheit das »Do not harm-Prinzip« und damit extraterritoriale Achtungspflichten der Kreditgeber empfindlich verletzen. Für die Misere der Staatsfinanzen tragen zwar vielfach die nationalen Regierungen die Hauptverantwortung. Auch entscheiden diese letztlich darüber, in welchen Bereichen wie viel gespart wird; vielerorts verschärfte jedoch der Druck internationaler Kreditgeber erheblich die Misere im Gesundheitswesen. Wenn die öffentliche Gesundheitsversorgung gar kollabiert, muss dringend gegengesteuert werden.

Während bereits die extraterritorialen Achtungspflichten viele Fragen aufwerfen, ist umstritten, inwiefern die Staaten darüber hinaus verpflichtet sind, andere Staaten bei der Umsetzung des Menschenrechts auf Gesundheit (und anderer sozialer Menschenrechte) zu unterstützen. Selbst Regierungen, die sich stark in der Entwicklungszusammenarbeit engagieren, möchten sich hierzu nicht völkerrechtlich verpflichten lassen. Gespannt darf man daher sein, welche Bedeutung die Maastrichter Grundsätze zu extraterritorialen Staatenpflichten im Bereich der wirtschaftichen, sozialen und kulturellen Menschenrechte entwickeln werden, die rund 40 Völkerrechtlerinnen und Menschenrechtsexperten aus aller Welt am 28. September 2011 an der Universität Maastricht verabschiedet haben. ${ }^{72}$ Die Prinzipien greifen Grundsätze auf, die UN-Ausschüsse und UN-Sonderberichterstatterinnen und -berichtserstatter mitunter bereits unverbindlich formuliert haben, und erkennen umfassende extraterritoriale Achtungs-, Schutzund Gewährleistungspflichten der Staaten an. ${ }^{73}$ Weiterreichend sind Forderungen nach »globalen sozialen Rechten«, welche die internationalen und transnationalen Beziehungen regulieren sollen. ${ }^{74}$

72 In deutscher Übersetzung abgedruckt sind diese in: Zeitschrift für Menschenrechte 6, 2 (2009), 184-195.

73 Vgl. auch Coomans/Künnemann (2012), de Schutter et al. (2012) und Krennerich (2013), 124-128.

74 Vgl. etwa Fischer-Lescano/Möller (2012). 


\section{Ausblick}

Sollen Menschenrechte Wirkung entfalten, müssen sie aktiv eingefordert werden. Aus diesem Grund ist menschenrechtliches Empowerment nötig. Dieses bezeichnet einen Prozess, in dessen Verlauf die Träger der Menschenrechte das Vermögen erlangen, die eigenen Menschenrechte und die Menschenrechte anderer effektiv einzufordern wie auch durchzusetzen. Hauptadressat menschenrechtlicher Ansprüche sind dabei die jeweiligen Staaten, welche die vornehmlichen Pflichtenträger sind. Deren Bereitschaft und Fähigkeit, die Menschenrechte umzusetzen, gilt es einzufordern und zu stärken. Aber auch nicht-staatliche Akteure wie Wirtschaftsunternehmen werden zusehends in die menschenrechtliche Verantwortung genommen. Den Betroffenen und ihren Unterstützergruppen steht dabei grundsätzlich ein breites Handlungsspektrum zur Verfügung, um ihre menschenrechtlichen Ansprüche gegen Widerstände zur Geltung zu bringen.

Aus juristischer Sicht zeichnet sich das Recht gerade dadurch aus, dass es sich auf dem Gerichtsweg durchsetzen lässt. Mit wachsender Anerkennung der - lange Zeit bestrittenen - Justiziabilität sozialer Menschenrechte $^{75}$ öffneten sich in den vergangenen Jahren vermehrt gerichtliche Klagemöglichkeiten. So ist das Case Law zu den sozialen Menschenrechten im Allgemeinen und zum Recht auf Gesundheit im Besonderen erheblich angewachsen. Inzwischen liegen zahlreiche Urteile und Entscheidungen vor, die das Recht auf Gesundheit direkt oder mittelbar betreffen. ${ }^{76}$ Interessanterweise beziehen sich diese nicht nur auf offenkundige Diskriminierungstatbestände und auf die Verletzungen der Achtungs- und Schutzpflichten, sondern mitunter auch auf Leistungsansprüche der Betroffenen. ${ }^{77}$ Empirisch ist allerdings stets zu prüfen, wer tatsächlich den Gerichtsweg

75 Vgl. Krennerich (2013), 116-123.

76 Vgl. die Datenbanken unter: www.globalhealthrights.org und www.escr-net.org [01.10.2015]. Siehe auch Gauri/Brinks (2008), Langford (2009), Yamin/Gloppen (2011), Hogerzeil et al. (2013) sowie das dreibändige Werk von Clérico et al. (2013).

77 Die deutsche Bundesregierung hält hingegen an der Rechtsauffassung fest, dass sich aus dem UN-Sozialpakt keine auf bestimmte Leistungen gerichteten Gewährleistungsansprüche ableiten lassen; vgl. Bundestagsdrucksache 18/4758, 27. April 2015. 
nutzt, wie die Gerichte entscheiden und ob entsprechende Urteile angemessen umgesetzt werden und nachhaltige Wirkungen zeitigen.

Mindestens ebenso wichtig ist, dass das Menschenrecht auf Gesundheit auf politischem Wege eingefordert und durchgesetzt wird, etwa über Proteste und Kampagnen oder über Lobby- und Advocacy-Arbeit. Bei aller Bedeutung einflussreicher gerichtlicher Leiturteile werden grundlegende, strukturelle Reformen, die darauf abzielen, die sozialen Menschenrechte, wie das Recht auf Gesundheit, besser und umfassender zu achten, zu schützen und zu gewährleisten, vor allem gesellschaftspolitisch erstritten, geht es hier doch hauptsächlich um Macht- und Verteilungskonflikte. Im Idealfall ergänzen sich dabei die (quasi-)gerichtliche Einklagbarkeit und die politische Einforderbarkeit des Rechts auf Gesundheit. Einerseits gewinnen politische Forderungen, Menschenrechte umzusetzen, dadurch an Legitimität und Schubkraft, dass die Rechtsansprüche positiv-rechtlich verankert sind und sich ggf. einklagen lassen. ${ }^{78}$ Andererseits werden die positiv-rechtliche Verankerung, Auslegung und Durchsetzung von Menschenrechtsnormen immer auch gesellschaftspolitisch erstritten und erwirkt. Hierfür ist in der Regel eine aktive Zivilgesellschaft von großer Bedeutung.

Inwieweit die Möglichkeiten, das Recht auf Gesundheit einzuklagen und einzufordern, genutzt werden, hängt zum einen von der Organisationskraft und der Handlungsfähigkeit zivilgesellschaftlicher Gruppen und sozialer Bewegungen ab. Zum anderen sind hierfür aber auch rechtsstaatliche Strukturen und zivilgesellschaftliche Freiräume vonnöten. Nicht wenige Regierungen beschneiden jedoch den Handlungsradius der Zivilgesellschaft und versuchen, menschenrechtliche Ansprüche zu unterbinden. Das Spektrum reicht hierbei von blanker Repression bis zur Kooptation. Dazwischen liegen offene und subtile Formen der Behinderung, etwa über rechtliche und administrative Einschränkungen der Versammlungs-, Vereinigungsund Meinungsfreiheit, oder auch die gezielte Diskreditierung, Stigmatisie-

78 Die bereits genannte Treatment Action Campaign (TAC) in der Republik Südafrika zeigte eindrucksvoll, wie sich politische und rechtliche Maßnahmen ergänzen können, um das Recht auf eine medizinische und medikamentöse Versorgung von HIV/AIDS-Patienten gegen massive Widerstände durchzusetzen; vgl. Heywood (2009). 
rung oder Kriminalisierung von Personen und Gruppen, die sich für die Menschenrechte einsetzen (im UN-Jargon: human rights defenders). ${ }^{79}$

Umso wichtiger ist es, zivilgesellschaftliches Engagement und menschenrechtliches Empowerment solidarisch $\mathrm{zu}$ organisieren und $\mathrm{zu}$ unterstützen. Hier liegt die große Bedeutung tatkräftiger transnationaler Menschenrechtsnetzwerke, welche die lokale und globale Ebene miteinander verbinden. Hinsichtlich des Rechts auf Gesundheit stellt beispielsweise die People's Health Movement ein solches globales Netzwerk dar. ${ }^{80}$ Dabei geht es wohlgemerkt nicht darum, vermeintlich westliche Menschenrechte in »fremde Länder« $\mathrm{zu} »$ exportieren«. Ausgangs- und Anknüpfungspunkte für eine solidarische Unterstützung sind immer die Kämpfe der Menschen vor Ort, die gegen erlittenes Unrecht, Unterdrückung, Ausbeutung und Not aufbegehren und sich - sei es implizit oder ausdrücklich - auf ihr Recht berufen, ein menschenwürdiges, freiheitliches und selbstbestimmtes Leben in Gemeinschaft mit anderen führen zu können. Das Menschenrecht auf Gesundheit gehört unbedingt dazu.

\section{LITERATUR}

Ach, Johann S. (Hg.) (2013): Grenzen der Selbstbestimmung in der Medizin, Münster: mentis.

Anliker, Nicole (2014): »Nie mehr Mutter sein. Zwangssterilisierungen in Peru«, in: Neue Zürcher Zeitung, 26. Dezember 2014.

BAfF (Bundesweite Arbeitsgemeinschaft der psychosozialen Zentren für Flüchtlinge und Folteropfer) (2015): Versorgungsbericht. Zur psychosozialen Versorgung von Flüchtlingen und Folteropfern in Deutschland, Berlin: BAfF.

Bahnsen, Ulrich (2015): »Unheimliche Enkel. Wenn Gentechniker vor Gentechnik warnen, sollten wir auf sie hören. Sonst könnte es zu spät sein«, in: Die Zeit, 19. März 2015, 1.

79 Vgl. Forum Menschenrechte (2012) sowie die Berichte der bisherigen Sonderberichterstatterinnen zu human rights defenders, abrufbar auf dem Menschenrechtsportal: www.ohchr.org [01.07.2015].

80 Vgl. etwa Turiano/Smith (2008) sowie www.phmovement.org [01.07.2015]. 
Bösl, Anton/Disecho, Joseph (Eds.) (2009): Human Rights in Africa, Windhoek: Konrad Adenauer Foundation.

Brot für die Welt/Misereor/ECCHR (2011): Transnationale Unternehmen in Lateinamerika: Gefahr für die Menschenrechte?, Stuttgart u.a.: BfdW u.a.

Burckhardt, Gisela (2014): Todschick. Edle Labels, billige Moden - unmenschlich produziert, München: Heyne.

Clérico, Laura/Ronconi, Liliana/Aldao, Martín (Cord.) (2013): Tratado de Derecho a la Salud, Tres tomos, Buenos Aires: Abeledo Perrot.

Coomans, Fons/Künnemann, Rolf (Eds.) (2012): Cases and Concepts on Extraterritorial Obligations in the Area of Economic, Social and Cultural Rights, Cambridge u.a.: Intersentia.

Cordier, Valeska (2015): »Zwangssterilisationen in Peru sollen strafrechtlich verfolgt werden«, in: Amerika 21. Nachrichten und Analysen aus Lateinamerika, 19. Februar 2015. Online: https://amerika21.de/2015 /02/112618/zwangssterilisationen-fujimori [26.06.2015].

De Schutter, Oliver/Eide, Asbjørn/Khalfan, Ashfaq/Orellana, Marcos/Salomon, Margot E./Seiderman Ian (2012): »Commentary to the Maastricht Principles on Extraterritorial Obligations of States in the Area of Economic, Social and Cultural Rights«, in: Human Rights Quarterly 34, 4 (2012), 1084-1169.

Deutsches Institut für Menschenrechte (Hg.) (2005): Die »General Comments « zu den VN-Menschenrechtsverträgen, Baden-Baden: Nomos.

Dodge, Rachel/Daly, Annette P./Huyton, Jan/Sanders, Lalage D. (2012): »The challenge of defining wellbeing «, in: International Journal of Wellbeing 2, 3 (2012), 222-235.

Engbruch, Katharina (2008): Das Menschenrecht auf einen angemessenen Lebensstandard, Frankfurt/M. u.a.: Lang.

Erices, Rainer/Frewer, Andreas/Gumz, Antje (2015): »Versuchsfeld DDR. Klinische Prüfungen westlicher Pharmafirmen hinter dem Eisernen Vorhang «, in: Frewer/Erices (2015), 129-143.

Fischer-Lescano, Andreas/Möller, Kolja (2012): Der Kampf um globale soziale Rechte, Berlin: Wagenbach.

Forum Menschenrechte (Hrsg.) (2012): Schützen statt verfolgen! Die schwierige Lage von Verteidiger/innen wirtschaftlicher, sozialer und kultureller Menschenrechte, Berlin: Forum Menschenrechte. 
Franke, Alexa (2006): Modelle von Gesundheit und Krankheit, Bern: Huber.

Freeman, Marsha A./Chinkin, Christine/Rudolf, Beate (Eds.) (2012): The UN Convention on the Elimination of All Forms of Discrimination Against Women. A Commentary, Oxford: Oxford University Press.

Frewer, Andreas/Erices, Rainer (Hg.) (2015): Medizinethik in der DDR. Moralische und menschenrechtliche Fragen im Gesundheitswesen, Geschichte und Philosophie der Medizin, Bd. 13, Stuttgart: Steiner.

Frewer, Andreas/Schmidt, Kurt W./Bergemann, Lutz (Hg.) (2013): Fehler und Ethik in der Medizin. Neue Wege für Patientenrechte, Jahrbuch Ethik in der Klinik, Bd. 6, Würzburg: Königshausen \& Neumann.

Fundamental Rights Agency (2012): Ungleichbehandlung und Mehrfachdiskriminierung im Gesundheitsbereich, Wien: FRA.

Gauri, Varun/Brinks, Daniel M. (Eds.) (2008): Courting Social Justice. Judicial Enforcement of Social and Economic Rights in the Developing World, Cambridge: Cambridge University Press.

Graf, Janna (2013): Weibliche Genitalverstümmelung aus Sicht der Medizinethik. Hintergründe - ärztliche Erfahrungen - Praxis in Deutschland. Mit einem Geleitwort von H. Bielefeldt und A. Frewer. Medizin und Menschenrechte, Bd. 6, Göttingen: V\&R unipress.

Graumann, Sigrid (2011): Assistierte Freiheit. Von einer Behindertenpolitik der Wohltätigkeit zu einer Politik der Menschenrechte, Frankfurt/M. u.a.: Campus.

Grodin, Michael A./Tarantola, Daniel/Annas, George J./Gruskin, Sofia (Eds.) (2013): Health and Human Rights in a Changing World, New York/London: Routledge.

Hestermeyer, Holger (2007): Human Rights and the WTO. The Case of Patents and Access to Medicines, Oxford: Oxford University Press.

Heywood, Mark (2009): »South Africa's Treatment Action Campaign. Combining Law and Social Mobilization to Realize the Right to Health«, in: Journal of Human Rights Practice 1, 1 (2009), 14-31.

Hogerzeil, Hans V./Samson, Melanie/Vidal Casanovas, Jaime/RahmaniOcora, Ladan (2013): »Is Access to Essential Medicines as Part of the Fulfillment of the Right to Health Enforcable through the Courts«, in: Grodin et al. (2013), 139-150.

Hunt, Paul/Backman, Gunilla (2013): »Health Systems and the Right to the Highest Attainable Standard of Health«, in: Grodin et al. (2013), 62-76. 
Jaichand, Vinodh/O'Donnell, Ciara (2010): »Bringing it home: The InterAmerican System and State Obligations Using a Gender Approach Regionally to Adress Women's Rights«, in: Inter-American and European Human Rights Journal 3, 1-2 (2010), 49-69.

Kälin, Walter/Künzli, Jörg (2008): Universeller Menschenrechtsschutz, 2. Aufl., Basel: Helbing Lichtenhahn Verlag.

Kaltenborn, Markus (2015): »Die Neufassung des Asylbewerberleistungsgesetzes und das Recht auf Gesundheit«, in: Neue Zeitschrift für Sozialrecht 24, 5 (2015), 161-166.

Keetharuth, Sheila B. (2009): »Major African Legal Instruments«, in: Bösl et al. (2009), 163-231.

Klee, Kristina (2000): Die progressive Verwirklichung wirtschaftlicher, sozialer und kultureller Menschenrechte, Stuttgart u.a.: Boorberg.

Krennerich, Michael (2013): Soziale Menschenrechte - zwischen Recht und Politik, Schwalbach/Ts.: Wochenschau Verlag.

Krennerich, Michael (2014): »Social Security - Just as much as a Human Right in Developing Countries and Emerging Markets«, in: Verfassung und Recht in Übersee 47, 1 (2014), 105-123.

Langford, Malcolm (Ed.) (2009): Social Rights Jurisprudence: Emerging Trends in International and Comparative Law, Cambridge: Cambridge University Press.

Lindner, Katja (2015): »Die gesundheitliche Versorgung von Asylsuchenden in Deutschland. Aktuelle politische Entwicklungen«, in: Migration und Soziale Arbeit, 1 (2015), 1-8.

Merten, Detlef/Papier, Hans-Jürgen (Hg.): Handbuch der Grundrechte in Deutschland und Europa, Band VI/2: Europäische Grundrechte II, Universelle Menschenrechte, Heidelberg: C.F. Müller.

Misbach, Elène (2015): »>Sich für die Gesundheit stark machen $<-$ Solidarische Flüchtlingsarbeit als gemeinsamer sozialer Kampf um Rechte«, in: Zeitschrift für Menschenrechte 9, 2 (2015), 122-135.

Morsink, Johannes (1999): The Universal Declaration of Human Rights. Origins, Drafting and Intent, Philadelphia: University of Pennsylvania Press.

Mylius, Maren/Bornschlegl, Wiebke/Frewer, Andreas (Hg.) (2011): Medizin für »Menschen ohne Papiere", Menschenrechte und Ethik in der Praxis des Gesundheitssystems. Medizin und Menschenrechte, Bd. 5. Göttingen: V\&R unipress. 
Mylius, Maren/Frewer, Andreas (2015): »Zugang zu medizinischer Versorgung von MigrantInnen ohne legalen Aufenthaltsstatus«, in: Zeitschrift für Menschenrechte 9, 2 (2015), 102-120.

Nettelsheim, Martin (2009): »Die Allgemeine Erklärung der Menschenrechte und ihre Rechtsnatur«, in: Merten et al. (2009), 191-236.

Nolan, Aife (2009): »Addressing Economic and Social Rights Violations by Non-State-Actors through the State: A Comparison of Regional Approaches to the >Obligation to Protect««, in: Human Rights Law Review 9, 2 (2009), 225-255.

People's Health Movement/Medact/Global Equity Gauge Alliance (2014): Global Health Watch 4. An Alternative World Health Report, London: Zed Books.

Platthaus, Andreas (2015): »Wer hilft mir in meinem Wahn? Der freie Patientenwille ist nicht alles. Manchmal hilft nur eine Zwangsbehandlung, etwa bei Schizophrenie. Odyssee eines Geisteskranken«, in: Frankfurter Allgemeine Zeitung, 10. Februar 2015, 9.

Saul, Ben/Kinley, David/Mowbray, Jacqueline (2014): The International Covenant on Economic, Social and Cultural Rights. Commentary, Cases and Materials, Oxford: Oxford University Press.

Schabas, William A. (Ed.) (2013): The Universal Declaration of Human Rights. The travoux préparatoires, 3 volumes, Cambridge: Cambridge University Press.

Schmidhuber, Martina (2013): »Überlegungen zu den Grenzen der Patientenverfügung für die Selbstbestimmung von Demenzbetroffenen im Anschluss an die Dworkin-Dresser-Debatte«, in: Ach (2013), 317-334.

Schmidhuber, Martina (2014): »Zum Personenstatus von Menschen mit Demenz«, in: Salzburger Jahrbuch für Philosophie LIV, 171-180.

Schneider, Jakob (2006): Menschenrechtlicher Schutz geistigen Eigentums, Stuttgart u.a.: Boorberg.

Shankar, Shyasri/Mehta, Pratap Bhanu (2008): »Courts and Socioeconomic Rights in India«, in: Gauri et al. (2008), 146-182.

Tobin, John (2012): The Right to Health in International Law, Oxford: Oxford University Press.

Turiano, Laura/Smith, Lanny (2008): »The catalytic synergy of health and human rights: The People's Health Movement and the Right to Health and Health Care Campaign«, in: Health and Human Rights 10, 1 (2008), 137-147. 
UNICEF (2002): Implementation Handbook for the Convention on the Rights of the Child, Fully Revised Edition, Geneva: United Nations Publications.

Venkatapuram, Sridhar (2011): Health Justice. An Argument from the Capabilities Approach, Cambridge/Malden, MA: Polity Press.

WHO (2008): Closing the Gap in a Generation: Health Equity through Action on the Social Determinants of Health. Final Report of the Commission on Social Determinants of Health, Online: www.who.int/social _determinants/final_report/csdh_finalreport_2008.pdf [01.06.2015].

Yamin, Alicia Ely/Gloppen, Siri (Eds.) (2011): Litigating Health Rights: Can Courts Bring More Justice to Health? Cambridge, MA: Harvard University Press.

Zenker, Heinz-Jochen (2011): »Europäische Strukturen der Gesundheitsversorgung von irregulären Migrantinnen und Migranten«, in: Mylius et al. (2011), 83-99.

Zimbabwe Human Rights NGO Forum (2009): »The right to health in Zimbabwe«, in: Human Rights Bulletin 41 (2009), Harare. 


\section{Das Recht auf Gesundheit in der Praxis}

Von der Forschung zur internationalen Therapie

ANDREAS FREWER

\section{EINFÜHRUNG ${ }^{1}$}

Menschenrechte sind grundlegende Freiheitsrechte. Für das Menschenrecht auf Gesundheit ${ }^{2}$ hat dies in Bezug auf Schutz und Sicherheit besondere Bedeutung: Die körperliche Unversehrtheit ist ein hohes Gut, das bei Angeboten zu medizinischer Diagnostik und Therapie oder wissenschaftlicher Forschung außerordentlich wichtig ist. ${ }^{3}$ Ohne eine differenzierte Aufklärung und die informierte Zustimmung eines entscheidungsfähigen Patienten ist jede Behandlung eine Körperverletzung. Dies betrifft in besonderem Maße alle Studien im Kontext medizinischer Forschung, aber auch allgemein die ärztliche Praxis. Vor 70 Jahren haben die Nürnberger Prozesse für die Medizin »Crimes against humanity ${ }^{4}$ in einem ungeheuerlichen Ausmaß offengelegt. ${ }^{5}$

1 Zu Vorarbeiten vgl. Frewer (2013) sowie Frewer et al. (2009b).

2 Zum Right to Health vgl. Jung (1982), Fritsche (1987), Toebes (1999), Gruskin et al. (2005), Ramm (2008), Seelmann (2008), UNHCR (2008), Grover (2013).

3 Vgl. van der Walt/Menke (2007) und insbesondere van der Walt (2007).

4 Diese Gräueltaten - häufig zu kurz mit »Verbrechen gegen die Menschlichkeit« übersetzt - sind durch die grundlegende Verletzung von Menschenrechten auch generell als »Verbrechen gegen die Menschheit « zu bezeichnen.

5 Vgl. Frewer (2000) und Frewer/Neumann (2001). Zum Nürnberger Ärzteprozess speziell Frewer et al. (1999) und Weindling (2004), 
Als Jadwiga Dzido, Überlebende der Versuche im Frauen-KZ Ravensbrück, mit ihren Aussagen und am eigenen Leibe die grausamen Experimente der Nazis bezeugen konnte, wurden immer mehr Details der menschenverachtenden Vorgehensweise von Häftlingsärzten und Forschungsleitern offenbart: Zahlreichen Widerstandskämpferinnen waren die Beine aufgeschnitten worden, um Kriegswunden zu simulieren und verschiedene Behandlungsarten auszuprobieren. ${ }^{6}$ Die medizinische Fragestellung ist zwar keine Pseudowissenschaft, denn Bombenverletzungen waren in dem durch die Nazis entfachten Weltkrieg an der Tagesordnung und angemessene Therapiewege umstritten, dennoch bedeutete diese Versuchsreihe ein menschenverachtendes Experiment ohne adäquate Schmerztherapie und mit hoher Mortalitätsrate. Der Nürnberger Ärzteprozess als Internationales Militärtribunal und erster Nachfolgeprozess hat letztlich in Reaktion auf dieses unmenschliche ärztliche Fehlverhalten mit dem »Nuremberg Code of Medical Ethics« im Urteilstext 1947 einen Katalog von zehn Voraussetzungen für »zulässige medizinische Versuche« verabschiedet.

Nach Ende des Zweiten Weltkriegs musste die Basis menschlichen Zusammenlebens neu geordnet werden. Die Universal Declaration of Human Rights aus dem Jahr 1948 versuchte ebenso ethische Grundlagen und Standards für Humanität neu zu verankern. Im Folgenden soll zunächst diese zentrale Dimension des Schutzes von Menschen - Probanden wie auch Patienten - in der Schnittmenge von Medizin und Menschenrechten anhand ihrer historischen Entwicklung rekonstruiert (Kap. 2) und in Bezug auf ethische Dimensionen an Grenzfragen erläutert werden (Kap. 3), bevor neben dem Schutz auf körperliche Unversehrtheit und dem nötigen »informed consent « zur Selbstbestimmung auch ein Blick auf die Praxis der Umsetzung (»Therapie«) des Rechts auf Gesundheit in internationaler Sicht geworfen wird (Kap. 4) und einige Schlussüberlegungen folgen (Kap. 5).

6 Vgl. zuletzt Genest (2015) sowie Schmidt (2006), Roelcke (2012), Weindling (2012) und Frewer/Schmidt (2014). Die Darstellung im Rahmen des Ärzteprozesses gewann zusätzliche Dramatik aus der Tatsache, dass andere Insassinnen des KZs freiwillig in den Tod gegangen waren, um durch den von den Widerstandskämpferinnen geheim eingefädelten Austausch das Überleben von Versuchsteilnehmerinnen zu erreichen. Zudem wurden von Betroffenen Dokumente vergraben, die erst kürzlich in deutscher Ausgabe erschienen: Genest (2015). 


\section{Patienten- Und Menschenrechte in der Medizin: ZENTRALE ENTWICKLUNGSETAPPEN}

Das paradigmatische Urteil für die deutsche Rechtsentwicklung in Bezug auf das Freiheitsrecht körperlicher Unversehrtheit wurde bereits vor rund 120 Jahren gefällt. Ein besonders problematischer Fall der Operation an einem siebenjährigen Kind hatte für grundlegende Kontroversen gesorgt: Bei diesem Mädchen sollte wegen eines tuberkulösen Fußes eine Amputation vorgenommen werden. Der Eingriff wurde zwar offensichtlich medizinisch korrekt durchgeführt, aber der erziehungsberechtigte Vater nicht um sein Einverständnis gebeten. Erstinstanzlich hatte das Hamburger Landgericht im Februar 1894 geurteilt, aber der Reichsgerichtshof revidierte im Mai desselben Jahres die Entscheidung: ${ }^{7}$ Nach seiner Auffassung war der Eingriff tatbestandlich als Körperverletzung zu werten. Die fehlende Einwilligung des Erziehungsberechtigten wurde grundlegend bemängelt. ${ }^{8}$ Letztlich sollten rechtlich nochmals die Wichtigkeit einer Unversehrtheit des menschlichen Körpers und die lediglich durch eine Einwilligung aufzuhebende Körperverletzung unterstrichen werden. Übergreifend waren damit sehr intensive rechtliche und moralische Diskussionen zu Nutzen und Schaden medizinischer Behandlung für Patienten verbunden, die natürlich bereits eine lange Tradition in Ärzteschaft und Medizingeschichte haben.

Das Prinzip des »Nichtschadens « wird schon seit der Antike und dem »hippokratischen Eid « ${ }^{9}$ vertreten; zwar besteht in Bezug auf dessen Verbreitung und Verbindlichkeit große Unsicherheit, aber er strebt ein hohes ärztliches Ethos und das Handeln zum Nutzen des Kranken an. Eine ausdrückliche Erwähnung der Autonomie von Patienten ist dort jedoch nicht

7 Entscheidungssammlung des Reichsgerichts in Strafsachen (RGSt) 25, 375, vgl. zudem u.a. Tag (2000).

8 Die genauen Details des Falls können an dieser Stelle nicht weiter ausgeführt werden; nach Rückverweis an das Landgericht wurde der angeklagte Arzt zwar vom Vorwurf der gefährlichen Körperverletzung freigesprochen, aber der Reichsgerichtshof hatte ein Grundsatzurteil gefällt.

9 Vgl. Edelstein (1969), Deichgräber (1983), Diller (1994) und Schubert (2005). Die Schreibweise »hippokratischer Eid« ist hier bewusst klein gewählt, um die (medizin)historisch wie philologisch umstrittene Autorschaft im Sinne eines »Eid des Hippokrates« zu hinterfragen. 
vorhanden. Im Altertum wurde das »primum nil nocere« (zuallererst nicht schaden) zu einem Eckpfeiler ärztlichen Handelns, das bis heute als Prinzip in der Medizinethik (»non-maleficence«) Geltung besitzt. ${ }^{10}$ Das Arztbild blieb jedoch über Jahrhunderte eher von einem "paternalistischen « ${ }^{11}$ Vorgehen der Heilenden geprägt. Damit ist ein väterlich-fürsorgliches Verhalten beschrieben, das im Zweifelsfall auch durch die ärztliche Autorität eines »Halbgottes in Weiß« bevormundend werden kann (»doctor knows best«) und dann das Recht des Patienten auf Selbstbestimmung zu missachten droht.

In besonderer Weise spielt die Autonomie des Kranken eine Rolle, wenn Interessen- und Zielkonflikte der Medizin zwischen der ärztlichen Rolle als Heiler und als Forscher zutage treten. Mit dem Streben nach wissenschaftlicher Erkenntnis rückte der Versuch am Menschen in das Blickfeld fortschrittlicher und ambitionierter Mediziner. Erste Formen wie etwa die Untersuchungen von Herophilos und Erasistratos im Wissenschaftszentrum Alexandria können bereits hierzu gerechnet werden: Dabei wurden laut Überlieferung auch Gefangene oder zum Tode verurteilte Menschen von diesen bedeutendsten Anatomen des hellenistischen Kulturraums als Studien- bzw. Lehrobjekte und sogar zu Vivisektionen benutzt. ${ }^{12}$

Die Genese der Menschenrechte und die Medizingeschichte sind allerdings bei weitem zu umfangreich und komplex, um in diesem Übersichtsbeitrag eine konzise Entwicklung der Patientenautonomie darstellen zu können; hier sollen nur wenige Meilensteine neuzeitlicher Medizin und die direkte Beziehung zu Menschenrechten und Ethik kurz vorgestellt werden. "Moralia et medicina haec sunt que unice aestimari debent«: Moral und Medizin sind es, die einzigartig zu schätzen sind, hatte Gottfried Wilhelm Leibniz (1646-1716) bereits im 17. Jahrhundert formuliert. ${ }^{13}$ Die Epoche der Aufklärung mit der "Declaration des droits de l'homme et du citoyen«

10 Vgl. Beauchamp/Childress (2009).

11 Hiermit ist der Arzt als »Vater« (pater) gemeint, der sich im Zweifelsfall eben auch »väterlich-bevormundend « verhält und den Willen des Patienten nicht ausreichend berücksichtigt. Siehe generell auch die Studie von Zude (2010).

12 Vgl. Toellner (1992), Bd. 1, 362; siehe auch Frewer/Oppitz et al. (1999), Ley/ Ruisinger (2001) und Frewer/Schmidt (2007). »Vivisektion« bezeichnet eine Eröffnung des Körpers bei lebendigem Leib.

13 Vgl. Leibniz (1976). 
(Frankreich 1789) war in der Folge eine zentrale Entwicklung für die Geistesgeschichte: Im hippokratischen Eidestext war seinerzeit sogar noch die Rede von Medizinern für Sklaven und Ärzten für Freie; die endgültige Anerkennung der Gleichheit aller Menschen und Bürger kann auch aus dieser Perspektive gar nicht hoch genug bewertet werden. Im Rahmen der Aufklärung gab es weitere positive Entwicklungen in Hinblick auf benachteiligte oder stigmatisierte Patientengruppen wie etwa für psychisch kranke Menschen die »Befreiung der Geisteskranken von ihren Ketten«.

Protagonisten des 18. Jahrhunderts hatten bereits die moralische Dimension und eine globale Verantwortung erkannt: »Handle so, daß du die Menschheit sowohl in deiner Person, als in der Person eines jeden andern, jederzeit zugleich als Zweck, niemals bloß als Mittel brauchst « formulierte Immanuel Kant (1724-1804) in seinem kategorischen Imperativ. ${ }^{14}$

In Bezug auf die Internationalität wurde bereits damals hervorgehoben: »Menschen sind Bürger eines allgemeinen Menschenstaats«, sie besitzen das »Weltbürgerrecht«, wie es der Königsberger Philosoph bereits in seinem »Ius cosmopoliticum « ausgedrückt hat. ${ }^{15}$ Kant antizipierte die globale Verantwortung:

»Da es nun mit der unter den Völkern der Erde einmal durchgängig überhandgenommenen (engeren oder weiteren) Gemeinschaft so weit gekommen ist, dass die Rechtsverletzung an einem Platz der Erde an allen [Plätzen] gefühlt wird, so ist die Idee eines Weltbürgerrechts keine phantastische und überspannte Vorstellungsart des Rechts, sondern eine notwendige Ergänzung des ungeschriebenen Kodex sowohl des Staats- als auch des Völkerrechts zum öffentlichen Menschenrecht überhaupt und so zum ewigen Frieden «. ${ }^{16}$

Gesundheit und Frieden wie auch »salus aegroti« und »salus publica« sind dabei oft zwei Seiten der gleichen Medaille. ${ }^{17}$ »Politik ist Medizin im Großen« fokussierte es der Arzt und Sozialpolitiker Rudolf Virchow (1821-

14 Kant (1900) IV, 429.

15 Vgl. Kant (1900).

16 Immanuel Kant, Zum ewigen Frieden. Ein philosophischer Entwurf (1795). Vgl. Anhang I, Über die Mißhelligkeit zwischen der Moral und der Politik, in Absicht auf den ewigen Frieden; siehe Kant (1900) sowie Höffe (2004).

17 Vgl. Schäfer et al. (2008). 
1902) im 19. Jahrhundert. ${ }^{18}$ Für die »Mikropolitik« von Wissenschaft und klinischer Versorgung gab es jedoch auch strukturelle Probleme und heikle Entwicklungen. Der französische Physiologe und Experimentalmediziner Claude Bernard (1813-1878) äußerte 1865: »[...] von den Experimenten, die man am Menschen ausführen kann, sind jene, die nur schaden können, verboten, jene, die nur harmlos sind, erlaubt, jene, die nützen können, geboten«. In der Praxis wurden aber immer mehr moralisch fragwürdige Praktiken am Patienten probiert. ${ }^{19}$ Die vielschichtige Eigendynamik wissenschaftlicher Arbeitsweise und die Fortschrittsorientierung der Epoche von »Mikrobenjägern« wird dabei illustriert durch die Einstellung Bernards, die Klinik könne nur als »Vorhalle«, das Labor aber als »Tempel« der Wissenschaft angesehen werden. ${ }^{20}$

Mit dem Aufstieg der Naturwissenschaften im 19. Jahrhundert zeigte sich dann deutlich eine Zunahme der ethischen Probleme in Bezug auf die Selbstbestimmung von Kranken: Einige Wissenschaftler betrachteten Patienten als bloße Verfügungsmasse (»corpus vile«) und meinten, über Kranke in ihrer Obhut frei verfügen zu können. Der Mediziner Bockhart etwa infizierte 1883 an Syphilis leidende todkranke Patienten noch zusätzlich mit Gonorrhoe, um die Geschlechtskrankheit Tripper in seinem natürlichen Verlauf und für wissenschaftliche Zwecke beobachten zu können. ${ }^{21}$ Der Arzt Schimmelbusch führte 1888 Versuche zu Infektionskrankheiten ebenfalls mit sterbenden Patienten durch. ${ }^{22}$

In besonderer Weise öffentlich diskutiert wurde - neben der Hamburger Kasuistik des Reichsgerichts 1894 - der Fall von Albert Neisser (18481912), Geheimrat und prominenter Professor für Hautkrankheiten an der Universitätsklinik Breslau. Anfang der 1890er Jahre hatte dieser eine »Serumtherapie« an acht Personen zur Impfung gegen die damalige Volksseuche Syphilis ausprobiert; dabei holte Neisser jedoch keinerlei Einwilligung

18 Vgl. u.a. Eckart (2013) sowie generell Klein/Menke (2004).

19 Diese These Claude Bernards geht auf sein Standardwerk »An Introduction to the Study of Experimental Medicine« aus dem Jahr 1865 zurück. Vgl. Bernard (1961), 147.

20 Ebd. Die Ehefrau Bernards war in der »Antivivisektionismus «-Bewegung gegen Tierversuche aktiv und trennte sich letztendlich von ihrem Mann.

21 Bockhart (1883).

22 Schimmelbusch (1888). 
der zum Teil sogar noch minderjährigen Patientinnen ein. Die Vakzinierung war letztlich nicht erfolgreich; in seiner eigenen Publikation bekannte der Geheimrat, dass in allen Fällen später eine Syphilis aufgetreten sei. ${ }^{23}$ In der liberalen Zeitung »Münchner Freie Presse« wurden in der Folge unter dem Titel »Arme Leute in Krankenhäusern« - wohl durch den Historiker und Pazifisten Ludwig Quidde (1858-1941) - kritische Berichte zum Fall Neisser anonym veröffentlicht. Nach kontroverser Diskussion um die Rechte von Patienten und Ärzten wurde Neisser in einem Disziplinarverfahren zu einer Strafe von 300 (Gold)Mark verurteilt und das Vorgehen damit klar als moralisch nicht legitim dargestellt. Eine zentrale Konsequenz war die Verabschiedung der »Preußischen Anweisungen« zur Forschung am Menschen am 29. Dezember 1900. In diesem wichtigen und frühen Dokument zur Formulierung von Patientenrechten wurde eine klare Unterscheidung zwischen medizinischer Grundlagenforschung und Heilversuchen gemacht. Außerdem schränkte man Studien für diagnostische, Heil- und Immunisierungszwecke an minderjährigen Personen oder Versuche an Sterbenden deutlich ein. Entscheidender Kern der Regulierung war, dass jede Person nach einer ärztlichen Belehrung ihr Einverständnis zu vorgesehenen Behandlungen geben muss. ${ }^{24}$ Albert Moll (1862-1939), Sexualwissenschaftler und Medizinethiker zu Beginn des 20. Jahrhunderts, dokumentierte und kritisierte diese Experimente im Rahmen einer großen Sammlung seines Buches »Ärztliche Ethik« mit hunderten Kasuistiken von Patientenmissbrauch durch die Medizin. $^{25}$

Größere öffentliche Debatten ähnlichen Inhalts entwickelten sich zudem bei Fällen mit kranken oder sterbenden Kindern; hierbei wurde Forschung in der Pädiatrie sogar in Form von Injektionen zur Untersuchung der Ruß-Verteilung in den Lungen terminal kranker Kinder durchgeführt, oder bei Ernährungsstudien mit Vitamin D »an einem Material von 100 Ratten und 20 Kindern « [sic] wurden Waisen absichtlich im Keller und fern von heilender Sonne gehalten. ${ }^{26}$ Besondere Aufmerksamkeit erregte dann

23 Vgl. u.a. Elkeles (1996) und Sabisch (2007).

24 Frewer/Schmidt (2007).

25 Moll (1902).

26 Das Zitat stammt aus einer Publikation der Forscher in der Deutschen Medizinischen Wochenschrift (1927). Zu den Debatten um Vigantol-Versuche in Berlin vgl. Frewer (2000), 114. 
Anfang der 1930er Jahre der »Lübecker Impfskandal«: 77 Kinder starben bei einer neu erprobten Schutzimpfung gegen Tuberkulose. Der Arzt und Gesundheitspolitiker Julius Moses (1868-1942), Reichstagsmitglied für die SPD, bezeichnete die Vorgänge als »Totentanz« und geißelte die »Experimentierwut« der Ärzte. Bereits vorher entworfene Richtlinien zu Humanversuchen wurden daraufhin vom Preußischen Innenministerium im Jahr 1931 endgültig verabschiedet. ${ }^{27}$ Auch dieser Text zur Wahrung von Patientenrechten konstatierte unmissverständlich das Primat der Selbstbestimmung des einzelnen Kranken vor den möglichen positiven Folgen der Forschung für das Gemeinwohl. ${ }^{28}$

Die bereits recht differenzierte Debatte während der Weimarer Republik konnte jedoch die Verbrechen gegen die Menschlichkeit während der Zeit des Nationalsozialismus nicht verhindern. Unerlaubte Experimente wurden in Kenntnis leitender Ärzte in einem ungeheuerlichen Ausmaß durchgeführt; unter Nazi-Medizinern starben viele »menschliche Versuchskaninchen«. Forschung in menschenverachtender Form wurde in KZs durchgeführt, so etwa in Dachau bei »terminalen Experimenten« zu Unterkühlung und Druckversuchen an russischen Gefangenen oder in Auschwitz an Zwillingen. ${ }^{29}$

Der eingangs erwähnte Nürnberger Ärzteprozess und der daraus entstandene Nuremberg Code of Medical Ethics (1947) versuchten in der Folge, Probanden zu schützen und Ärzte von der Durchführung unmoralischer Experimente abzuhalten. Kurz darauf wurde die Declaration of Geneva (1948) durch die World Medical Association (WMA) verabschiedet mit der direkten Bezugnahme auf die hippokratischen Ideale des antiken Eides, um Patienten vor Missbrauch zu schützen. Dieses »Genfer Gelöbnis« wurde als Präambel verpflichtender Teil der Musterberufsordnung für deutsche Ärzte. Für das Gebiet der Humanversuche dauerte es jedoch noch einige Jahre, bis der Weltärztebund in der Declaration of Helsinki

27 Siehe Tröhler/Reiter-Theil (1998), Frewer (2000) und Bonah et al. (2003).

28 Vgl. Frewer/Neumann (2001) und Reich (1995), 2762-2763.

29 Siehe auch Frewer et al. (1999) und Eckart (2013). Auf internationale Entwicklungen - etwa menschenverachtende japanische Versuche in China - kann hier nicht eingegangen werden, siehe auch Schmidt/Frewer (2007) sowie Council for International Organizations of Medical Sciences (CIOMS) (1993), (2002) und (2009) sowie Erices et al. (2014) und Lehner/Frewer (2014). 
$(1964)^{30}$ die Grundsätze der Forschung am Menschen weiter spezifizierte, wenn auch manche Nürnberger Kriterien wieder etwas abgeschwächt wurden. Mit der Novellierung von Tokio (1975) sowie Ergänzungen in Edinburgh (2000), Washington (2002), Seoul (2008) und zuletzt im brasilianischen Fortaleza (2013) wurde dieses Dokument ein zentraler Text zur Regulierung von Forschung. Trotzdem ereigneten sich auch nach dem Zweiten Weltkrieg - sogar in demokratischen Staaten wie den USA oder England - Fälle, die erneut die Frage ethischer Grenzen aufwarfen. Dabei standen militärische Forschung wie etwa die Strahlen-Versuche mit USSoldaten ${ }^{31}$ oder die »Tuskegee Syphilis-Experimente ${ }^{32}$ an farbigen Amerikanern im Brennpunkt ethischer Debatten. ${ }^{33}$ Gesundheit als Menschenrecht hat hier eine besondere Bedeutung zum Schutz des Patientenwohls; damit sind auch Einschränkungen für forschende Mediziner und die klare Trennung von Interessen der Wissenschaft oder des Staates im Kontrast zu Patientenrechten vorhanden.

\section{Menschenrechte und Patientenautonomie IN GRENZBEREICHEN}

Die historischen Ereignisse im Kontext von Nationalsozialismus und Zweitem Weltkrieg haben in Deutschland zur verfassungsrechtlichen Verankerung von Grundrechten geführt. ${ }^{34}$ Diese sind auch Basis des Selbstbestim-

30 Zur Verbindung der Deklaration von Helsinki mit Menschenrechtsfragen gibt es eine eigene Diskussion, siehe u.a. Plomer (2004) und Frewer/Schmidt (2014) sowie ferner Andreopoulos (2000).

31 Siehe insbesondere Advisory Committee on Human Radiation Experiments (1996) und Moreno (2000).

32 Vgl. u.a. Jones (1981).

33 Vgl. generell Tröhler/Reiter-Theil (1998) und Schmidt/Frewer (2007). Zu nennen wären in diesem Kontext auch die Initiativen zu »Patients‘ Bill of Rights« seit den 1970er Jahren, siehe u.a. Klein/Menke (2004) und Marks (2006).

34 Auf das Ausmaß der »Medizin ohne Menschlichkeit« kann hier nicht weiter eingegangen werden: Neben so genannter »reiner Forschung « gab es eine Reihe weiterer Menschenrechtsverletzungen in der Medizin wie die rassistische Diskriminierung, Zwangssterilisationen und »Euthanasie«; letztere diente sogar 
mungsrechts von Patienten: Das Grundgesetz formuliert im ersten Absatz von Artikel 1 die Menschenwürde als entscheidende Fundierung des $\mathrm{Zu}-$ sammenlebens von Bürgern in einem Staat. ${ }^{35}$ Die anschließenden Passagen unterstreichen die Persönlichkeitsrechte (Art. 2 Abs. 1 GG) und insbesondere auch das für die Gesundheit zentrale Recht auf körperliche Unversehrtheit (Art. 2 Abs. 2 GG). Freiheitsrechte des Einzelnen werden hier gerade für das Recht auf Gesundheit geschützt. In Übertragung auf die Probleme der Medizin kann zunächst festgehalten werden: Verweigert oder widerruft ein Patient die Einwilligung, ist eine (weitere) Behandlung moralisch und rechtlich nicht legitim. Dies soll im Folgenden an ausgewählten Beispielen kurz skizziert und exemplarisch erklärt werden. ${ }^{36}$ Vor einem Eingriff oder einer Operation müssen Aufklärung und Einwilligung zwischen Patient und Arzt gewährleistet sein; mit dem Nürnberger Kodex ist die Notwendigkeit der freiwilligen Zustimmung - seinerzeit noch als »voluntary consent « - im Sinne der zentralen Forderung eines »informed consent« unterstrichen worden. Dies kann im klinischen Alltag - z.B. bei einer Blutentnahme - im beiderseitigen mündlichen oder auch stillschweigend-handelnden Einverständnis erfolgen; vor größeren Eingriffen zu diagnostischen oder therapeutischen Zwecken steht eine schriftliche Erklärung

makabrer Weise als »Versuchslabor« für den europäischen Völkermord und den Holocaust. Nahezu alle Paragraphen der AEMR wurden im NS-Staat verletzt, die Medizin hat sich bei vielen Aspekten beteiligt: Gegen Artikel 5 - »Niemand darf einen anderen Menschen quälen, erniedrigen oder grausam bestrafen« wurde millionenfach und in besonders schlimmer Form gerade durch Mediziner verstoßen. Das Verbot von Sklaverei und Sklavenhandel (Artikel 4) wurde auch im Gesundheitswesen und in der Medizin mit der flächendeckenden Beschäftigung von Zwangsarbeitenden gebrochen, vgl. u.a. Frewer/Siedbürger (2004). Gerade das Primat des NS-Kollektivwohls (»Volkskörper«/»Rasse«) gegenüber grundlegenden Rechten des Einzelnen war für die »Ethik« und ihre grausamen Konsequenzen 1933-1945 entscheidend; vgl. Frewer (2000).

35 Zur neueren Diskussion um den vielschichtigen Begriff der Menschenwürde mit Bezug zu Ethik und Recht in der Medizin vgl. u.a. Joerden et al. (2011).

36 Im Folgenden können die breiten Debatten zu einzelnen Themenfeldern nur kurz genannt und nicht vertieft werden; zu weiterer Literatur siehe auch Reich (1995) und Maio (2012). 
des Patienten, der über Risiken und Nebenwirkungen geplanter Behandlungen vollständig aufgeklärt werden muss.

Konflikte entstehen, wenn Patienten eine Therapie - etwa Zeugen Jehovas eine Bluttransfusion - verweigern. Die Verabreichung gegen den Willen eines entscheidungsfähigen Patienten ist rechtlich unzulässig; sie stellt eine Verletzung der körperlichen Integrität dar und ist zudem durch die in Artikel 4 des Grundgesetzes geschützte Religionsfreiheit abgedeckt. ${ }^{37}$ Das Gemeinwesen schützt hierbei die Freiheit religiöser Wertsetzung, auch wenn das nicht im Sinne der Gesundheit des einzelnen Patienten ist. Noch schwieriger wird das Beispiel, wenn Eltern eines Kindes eine sinnvolle Behandlung aus religiösen Gründen verweigern. Bis zum 14. Lebensjahr gelten Kinder in der Regel als nicht einwilligungsfähig, auch wenn sie - je nach konkretem Reifegrad im Einzelfall - bereits sehr viel verstehen können und in Entscheidungsprozesse nach Möglichkeit eingebunden werden sollten. An ihrer Stelle entscheiden die Eltern als Sorgeberechtigte bei Eingriffen oder Operationen. Verweigern Erziehungsberechtigte die Einwilligung zu einer ärztlich indizierten Maßnahme - in diesem Fall eine lebensrettende Bluttransfusion - so kann hierdurch eine erhebliche oder gar vitale Gefährdung des Kindeswohls resultieren. Von ärztlicher Seite kann zur Abwehr einer Kindeswohlgefährdung ein Betreuungsgericht (früher: »Vormundschaftsgericht«) angerufen werden. Bei elektiven, also planbaren Eingriffen ohne Notfall sollte selbstverständlich im Vorfeld auf die Möglichkeit zur Behandlung durch andere Kliniken, die auf besonders blutsparende Operationstechniken oder den Ersatz von Fremdblut durch andere Substanzen spezialisiert sind, hingewiesen werden. Diese Fälle verlangen von allen Beteiligten hohen kommunikativen Einsatz; für das ärztliche Gewissen und die Klinische Ethikberatung entstehen hierbei häufig besonders heikle Konflikte. Zeugen Jehovas haben mit umfangreichem Informationsmaterial und der Einrichtung von Verbindungskomitees in Krankenhäusern auf diese Probleme reagiert. ${ }^{38}$

Die Konstellation, dass Eltern eines Kindes eine sinnvolle Operation verweigern, kann selbstverständlich auch in anderen Formen vorkommen; so gab es sogar bereits gegen den Willen der Eltern und mithilfe der Polizei

37 Vgl. u.a. Wachtturm Bibel- und Traktatgesellschaft (2000) und Stiftung kreuznacher diakonie, Der Vorstand/Ethikausschuss (2011).

Ebd. 
einer sinnvollen Therapie zugeführte Kinder, etwa wenn Eltern bei pädiatrischen Tumorerkrankungen irrationalen esoterischen Behandlungsformen zuneigen und ihren Schutzbefohlenen notwendige und risikoarme Eingriffe vorenthalten. Zuletzt hat der Bundesgerichtshof etwa im Fall des »Guru von Lonnerstadt«, der einem Kind mit Mukoviszidose alternative Behandlungsformen statt der bewährten Therapien zukommen ließ, mit der Verurteilung des esoterischen Heilers nicht bewährter Alternativmedizin klare Grenzen gesetzt. ${ }^{39}$ Generell steht hier das Kindeswohl im Sinne des »best interest standard « im Vordergrund einer kritischen Abwägung.

Fragen der Selbstbestimmung können aber auch in der umgekehrten Weise als Problem relevant werden, etwa wenn zum Beispiel Patienten einen medizinisch sinnlosen Eingriff oder falsche Operationen wünschen. Diese Konstellation liegt in besonderer Form vor beim Krankheitsbild der »Body Integrity Identity Disorder« (BIID): ${ }^{40}$ Eine Person möchte dabei vom Arzt die Veränderung des Körpers, teils in derart extremer Form, dass zum Beispiel die Entfernung eines Beins oder gar die Durchtrennung des Rückenmarks gewünscht wird. Das spezifische Körperbild des betreffenden Menschen sieht dabei offenbar eine andere Erscheinungsform vor und führt häufig zu gravierenden psychischen Problemen. Während viele Ärzte derartigen Wünschen verständnislos oder ablehnend gegenüber stehen, gab es auch bereits eine Reihe umstrittener Eingriffe. Dabei entstehen durchaus nicht nur individuell ganz spezifische Konstellationen für Patienten, sondern auch Konsequenzen mit sozialen Belastungen für das Gemeinwesen, wenn das Selbstbild eines Menschen eine bestimmte Behinderung vorsieht und der Betreffende etwa ein Leben im Rollstuhl anstrebt. In gewisser

39 Der BGH hat entschieden, dass eine Mutter und ihr Lebensgefährte (der »Guru«) wegen Misshandlung eines schwer kranken Kindes für drei Jahre ins Gefängnis müssen. Rechtlich wurde argumentiert, das Paar sei seiner Pflicht nicht nachgekommen, dem seinerzeit 13jährigen Sohn der Frau die notwendige und bestmögliche medizinische Behandlung zukommen zu lassen. Das Paar entschied sich aus esoterischer Überzeugung, die notwendige Behandlung der Stoffwechselkrankheit (insbesondere verbunden mit starker Verschleimung der Atemwege) abzubrechen; stattdessen versuchten sie alternative Heilmethoden und Meditation (Az.: 1 StR 624/14). Vgl. www.sueddeutsche.de/bayern/bghurteil-guru-von-lonnerstadt-muss-drei-jahre-in-haft-1.2594459 [10.12.2015].

40 Vgl. insbesondere Stirn et al. (2010) und Manok (2012). 
Weise grenzen diese Fälle auch an eine generelle Strömung so genannter »wunscherfüllender Medizin «: ${ }^{41}$ Patienten werden in der modernen und zunehmend kommerzialisierten Medizin der Gegenwart immer stärker zu »Kunden«, die als nachfragende Verbraucher auch die Angebote auf dem Gesundheitsmarkt beeinflussen. ${ }^{42}$ Dies reicht von »Individuellen Gesundheitsleistungen« (IGeL) mit Aufpreis bis hin zu erhöhter Frequenz von lukrativen diagnostischen Eingriffen (z.B. Herzkatheter-Untersuchungen) oder im Einzelfall auch Operationen - (kosmetische) Chirurgie ohne erforderliche Indikation - im System fallbezogener Vergütung ärztlicher Leistungen nach Diagnosis related Groups (DRGs) oder bei Privatpatienten.

Ein Grenzbereich mit hoher sozialer Relevanz ist hierbei am Lebensbeginn zu sehen: Führen die Möglichkeiten von In-vitro-Fertilisation, Pränatal- oder gar Präimplantationsdiagnostik langfristig zu »Wunschkindern nach Wahl«? Hier ergeben sich breite Debatten zur sozialen Gestaltung von »Anthropotechniken« und neuen Formen einer »Eugenik von unten«. Während Berichte über »Dr. med. Klon«, den italienischen Mediziner Antinori, ${ }^{44}$ der einen weltweit ersten Menschen-Klon herstellen wollte, oder über den südkoreanischen Stammzell-Forscher Hwang, ruhmsüchtige Ankündigungen, Fälschungen oder Manipulationen nachweisen, kann an der generellen Möglichkeit zur Herstellung von Leben wie auch gentherapeutischer Eingriffe kein Zweifel mehr bestehen. ${ }^{45}$

Ethische Fragen der Patientenautonomie und informationeller Selbstbestimmung stellen sich von den Grundlagen der Genetik über die Synthetische Biologie bis hin zur Speicherung in Datensystemen und Biobanken. Die Organisation der Vereinten Nationen für Erziehung, Wissenschaft und

41 Zur Gliederung in vier Modelle der Patient-Arzt-Beziehung - »paternalistisches«, »informatives«, »interpretatives « und »deliberatives« Konzept - vgl. Emanuel/Emanuel (1992) und zur Erweiterung auf sechs Formen (ergänzt um »autoritäres« Arztverhalten und »wunscherfüllende Medizin« für Kunden) siehe Kettner/Kraska (2009) sowie Kettner (2009) und Eichinger (2013).

42 Vgl. u.a. Frewer et al. (2011).

43 Siehe insbesondere Habermas (2001) und UNESCO (2005).

44 Vgl. u.a. Bahnsen (2001). Oft wird in diesem Kontext (»reproduktive Freiheit«) sogar von einem »Menschenrecht auf ein Kind « gesprochen, was aber in dieser Form nicht zu konstituieren ist.

Vgl. u.a. CIB/DPA (2010). 
Kultur (UNESCO) verabschiedete im Jahr 2005 die »Universal Declaration on Bioethics and Human Rights«, in der auf Menschenrechtsfragen und insbesondere das notwendige Maß an Autonomie von Patienten im Zeitalter von Genomanalyse und personalisierter Medizin speziell eingegangen wird. $^{46}$

Ethische Fragen von ebenso großer sozialer Relevanz ergeben sich im Spannungsfeld von Selbstbestimmung und Patientenwohl am Lebensende. In Bezug auf die menschenrechtliche Dimension hat der Fall einer Patientin mit Amyotropher Lateralsklerose (aufsteigende Lähmung) für größere Debatten mit Bezug zu menschenrechtlichen Fragen der Medizin gesorgt: Die 43jährige Britin Diane Pretty hatte gefordert, dass Tötung auf Verlangen (Killing on demand) als ein Menschenrecht anerkannt werden sollte. Nach Scheitern vor englischen Gerichten und mit einer Petition an den Premierminister Tony Blair zog die Frau vor den Europäischen Gerichtshof für Menschenrechte in Straßburg. Dieser sah im Ergebnis durch die verweigerte Sterbehilfe letztlich keine Verletzung der Menschenrechte; die angemahnten Privat- und Freiheitsrechte wurden von den Richtern als nicht verletzt angesehen. Die Engländerin starb im Übrigen kurz darauf friedlich in einem Hospiz in der Nähe von London. ${ }^{47}$ In Deutschland gilt seit dem Herbst 2009 das Gesetz zur Patientenverfügung (Änderung im Betreuungsrecht); auf diese Weise ist der Stellenwert autonomer Entscheidungen von Kranken nochmals unterstrichen worden. ${ }^{48}$ Im November 2015 wurde zuletzt eine Novellierung des § 217 StGB verabschiedet und kommerzielle Suizidhilfe verboten. ${ }^{49}$

An vielen der genannten Beispiele wird klar, dass der Passus vom »Menschenrecht auf Gesundheit« zweierlei meint: Es handelt sich einerseits um das Recht, von bestimmten Formen medizinischer Eingriffe oder wissenschaftlicher Forschung im Rahmen selbstbestimmter Entscheidungen verschont zu bleiben. Aus diesem Grund bezeichnet man sie auch als »negative« Rechte, da es um das Unterlassen von Handlungen geht. Anderer-

46 Vgl. UNESCO (2005).

47 Siehe etwa die Berichte im Deutschen Ärzteblatt (2002) sowie generell Schäfer et al. (2012).

48 Vgl. Frewer et al. (2009).

49 Vgl. u.a. Richter-Kuhlmann (2015). Überdies wurden bereits kurz zuvor Hospizund Palliativversorgung gestärkt, vgl. Beerheide/Richter-Kuhlmann (2015). 
seits gibt es das »Menschenrecht auf Gesundheit« als positives Anspruchsrecht auf angemessenen Zugang zu höchstmöglichen Leistungen der Gesundheitsversorgung, die natürlich ständiger Entwicklung unterliegt. In dieser Hinsicht differiert es international in hohem Maße und ist kaum bezifferbar: Es handelt sich dabei in der Regel um das Recht auf angemessene medizinische Versorgung, die je nach Standort des Patienten auf dieser Erde in ganz unterschiedlicher Weise realisiert werden kann. ${ }^{50}$ Dabei stellen sich selbstverständlich ethische Probleme der Gerechtigkeit und globaler Allokation: Wie lässt sich eine transnationale Solidaritätsgemeinschaft im Sinne des Weltbürgertums erreichen?

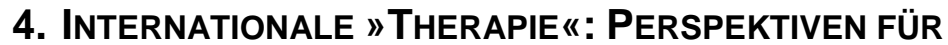 PATIENTEN- UND MENSCHENRECHTE IN DER PRAXIS}

Nicht nur das Feld der Forschung in der Medizin hat sich in der Geschichte der Menschheit im Sinne von »Trial and Error« fortentwickelt, sondern auch die allgemeinen Anstrengungen, im Gesundheitswesen der einzelnen Länder sukzessive Verbesserungen zu erreichen, können als wiederholte Versuche verstanden werden, durch die Medizin immer bessere Bedingungen für den Einzelnen wie auch die (globale) Gesellschaft zu erreichen. ${ }^{51}$ Internationalen Deklarationen sind dabei der immer wieder erneuerte Anlauf, allgemeingültige Standards im Konsens zu vereinbaren und damit die Praxis im Sinne von Gleichheit und Gerechtigkeit voranzubringen. $^{52}$

50 Hierbei sind unterschiedliche Grade der Rechtsverwirklichung zu unterscheiden. Das Recht auf Gesundheit meint nicht nur »minimale Gesundheit«, sondern das Maximum an erreichbarer Gesundheit für alle. Die »minimum core obligations « decken den Gesamtumfang des Rechts keineswegs ab, sondern beschreiben diejenigen Komponenten, die eigentlich - ohne Ressourcenvorbehalt - unmittelbar umgesetzt werden müssen und ggf. auch einklagbar sind. Siehe auch den Beitrag von Müller im vorliegenden Band.

51 Zur Debatte, welche Pflichten des Einzelnen in Bezug auf Menschenrechte bestehen, vgl. u.a. Clapham (2006).

52 Da es bei Mindeststandards ebenso um eine »Unversehrtheit des Körpers« geht, gibt es einen direkten Bezug zum vorangehenden Abschnitt. 
Artikel 25 der Allgemeinen Erklärung der Menschenrechte der Vereinten Nationen stellt fest: »Jeder Mensch hat Anspruch auf Unterstützung, wenn er in Not geraten ist.« Medizin hilft Menschen, die in Krisen geraten sind und Unterstützung brauchen, Gesundheit ist das zentrale Gut mit weltweiter Relevanz und Notwendigkeit. Der Artikel geht aber noch weiter: »Wenn Menschen nicht arbeiten können, weil es keine Arbeit gibt, sie krank sind oder zu alt, muss ihnen geholfen werden. Mütter und Kinder haben Anspruch auf Schutz.«

Ein zentraler Meilenstein zur Annahme des Rechts auf Gesundheit ist die mittlerweile ein halbes Jahrhundert zurückliegende Formulierung der »International Convention on Economic, Social and Cultural Rights« (1966), die zehn Jahre später in Kraft getreten ist. ${ }^{53}$ Bei der weiteren Ausarbeitung der wirtschaftlichen, sozialen und kulturellen Rechte (kurz: WSK-Rechte) sind sukzessive Fortschritte gemacht worden, wobei der »General Comment No. 14« des UN-Ausschusses für wirtschaftliche, soziale und kulturelle Rechte (WSK-Ausschuss) ausdrücklich auch das Recht anspricht, über die eigene Gesundheit und den eigenen Körper zu bestimmen, einschließlich der sexuellen und reproduktiven Freiheit, das Recht, frei von Eingriffen zu sein und das Recht, nicht misshandelt, nicht medizinischer Behandlung oder medizinischen Versuchen ohne Einwilligung unterzogen zu werden.

Im gleichen Jahr 2000 einigten sich 189 Mitgliedsstaaten der Vereinten Nationen (UN) auf grundlegende Werte internationaler Zusammenarbeit und spezielle Ziele für die gesamte Menschheit. Eine Erklärung bezüglich der »Millennium Development Goals« (MDG) unterstrich den Willen der Völkergemeinschaft, eine Situation globalen Friedens zu erreichen, Armut zu bekämpfen und eine nachhaltige Förderung der Entwicklung zu schaffen, die Umwelt zu schützen sowie die Menschenrechte zusammen mit den Grundsätzen der Demokratie und guter Regierungsführung zu achten. ${ }^{54}$ Auch bei diesen seit der Jahrtausendwende anvisierten Absichten rangiert die Gesundheit an zentraler Stelle. Unter den acht genannten Zielen beziehen sich mehrere auf Ernährung, Kinder- und Müttersterblichkeit sowie die Vermeidung der Übertragung ansteckender Krankheiten und somit direkt

53 Die Schwelle für das Inkrafttreten war eine Ratifikation durch 35 Staaten. Obwohl diese Hürde nicht sehr hoch war, hat es doch zehn Jahre gedauert.

54 Vgl. www.un.org/millenniumgoals/ [26.01.2015]. 
auf das Recht auf ein höchstmögliches Maß an Gesundheit. Diese Millenium-Entwicklungsziele sahen im Einzelnen folgende Schwerpunkte vor: 1. Extreme Armut und Hunger sollten bis $2015^{55}$ halbiert werden, ebenso der Anteil der Menschen, die mit weniger als einem US-Dollar pro Tag (in reicheren Ländern zwei Dollar) überleben müssen. Der Anteil der hungernden Menschen sollte ebenfalls mindestens halbiert werden. 2. Allen Kindern sollte der Besuch einer Primarschule ermöglicht werden. 3. Eine Gleichstellung der Geschlechter soll gefördert werden, insbesondere bei der Primar- und Sekundarschulbildung. 4. Die Sterblichkeitsrate von Kindern unter fünf Jahren sollte bis 2015 um zwei Drittel gesenkt werden. 5. Die Sterblichkeitsrate von Müttern sollte bis 2015 um drei Viertel gesenkt werden. 6. Mit Armut verbundene Krankheiten sollten konsequent bekämpft werden. Dabei wird ein besonderer Schwerpunkt auf Kinder- und Müttersterblichkeit sowie auf Immunkrankheiten gelegt. Die Ausbreitung von HIV/AIDS ${ }^{56}$ soll bis 2015 gestoppt werden. 7. Die ökologische Nachhaltigkeit soll gesichert werden, indem der Zugang Benachteiligter zu Ressourcen wie Trinkwasser, Land und Wald verbessert, die Entwicklung von Slums in Städten zurückgeführt und erneuerbare Energien verstärkt und zum Nutzen der Armen eingesetzt werden. 8. Eine globale Entwicklungspartnerschaft soll aufgebaut werden. Hierzu werden vor allem bessere Welthandelsbedingungen angestrebt, die Entschuldung der Entwicklungsländer vorangetrieben, mehr und effektivere Entwicklungsfinanzierung bereitgestellt und eine bessere Partnerschaft mit besonders benachteiligten Ländern entwickelt. Im Rahmen des Milleniumsprojektes zu Entwicklungszielen wurde 2005 ein weiterer Aktionsplan publiziert; ${ }^{57}$ ein spezieller Bericht des UN-Generalsekretärs mit dem Titel »In Larger Freedom: Towards Development, Security and Human Rights for All« bezog sich dabei explizit auf die Weiterentwicklung der Menschenrechte. ${ }^{58}$

55 Hier ist eine Halbierung gegenüber dem Stand im Jahr 1990 gemeint.

56 Vgl. u.a. Macklin (2004) und (2010).

57 »Sachs Report«. Vgl. www.un.org/Depts/german/wirtsozentw/sachsreport.pdf [10.12.2015].

58 Auf den Prozess zur Weiterentwicklung der »Post-Millennium Development Goals« 2015, in dem Gesundheit häufig unterrepräsentiert erscheint, kann hier nicht weiter eingegangen werden; dazu wird in Folgebänden der vorliegenden Reihe detaillierter Stellung genommen. 
Ärztinnen und Ärzte, Pflegende und Mitarbeiter im Gesundheitswesen versuchen international, die strukturell vorhandene oder sich akut immer wieder verschärfende Not zu lindern. Eine Fülle von Ärzte- und Hilfsorganisationen wie etwa das Internationale Rote Kreuz oder der Rote Halbmond, Medécins sans frontières (Ärzte ohne Grenzen), medica mondiale, Ärzte in sozialer Verantwortung oder Mediziner und Psychologen bei Amnesty International arbeiten daran, das Menschenrecht auf gesundheitliche Versorgung für Betroffene und Opfer in der praktischen Arbeit umzusetzen. Die Medizin ist dabei keineswegs nur auf die ökonomische Seite der Menschenrechte und globale Gerechtigkeit konzentriert, es ergibt sich eine Reihe weiterer Aspekte und Problemfelder: Mit den Anstrengungen zu einer »Universellen Gesundheitssicherung " (Universal Health Coverage) durch die Weltgesundheitsorganisation ${ }^{59}$ oder die verbindliche Formulierung von Kernpflichten bzw. Mindeststandards (»Minimum Core Obligation « ${ }^{60}$ innerhalb des Menschenrechts auf Gesundheit auf internationaler Ebene sind zwei zentrale Initiativen genannt. Letztlich muss die Bevölkerung der Erde - langfristig wie auch nachhaltig - als "globale Solidaritätsgemeinschaft« verstanden werden.

Dies war auch das Ziel einer Gruppe von Wissenschaftlern, deren Überlegungen zur praktischen Umsetzung im so genannten »Kopenhagener Konsens« formuliert wurden. Dabei sollten globale Prioritäten im Sinne einer Weltinnenpolitik gesetzt werden: Es wurde ein »30-Punkte-Plan zur Rettung der Welt« mit Lösungen für die Probleme von Armut und Gesundheit gesucht, die mit möglichst effektiver Mittelverwendung angegangen werden sollen. Eine Expertengruppe aus den Wirtschaftswissenschaften analysierte über zwei Jahre die Lage. ${ }^{61}$ Acht führende Ökonomen, darunter

59 Siehe insbesondere den Beitrag von Heinicke, Eriksson, Saxena und Reis im vorliegenden Band.

60 Vgl. speziell den Aufsatz von Müller in diesem Buch. Zu Fragen der Mindeststandards zur Forschung in Entwicklungsländern siehe zudem u.a. Participants in the 2001 Conference on Ethical Aspects of Research in Developing Countries (2004) sowie Macklin (2006).

61 Ursprünglich wurde das Projekt 2004 durch den Professor für Statistik Bjørn Lomborg und andere Mitglieder des »Institute for Environmental Assessment«, eine Stiftung der dänischen Regierung, initiiert sowie mitfinanziert durch das 
mehrere Nobelpreisträger, formulierten eine Agenda mit Vorschlägen zur Verbesserung der Situation der Menschheit. Unter dem Titel Kopenhagen Konsens sind ihre Konzepte im Sommer 2008 - noch vor dem Beginn der Finanzkrise und deren harten globalen Auswirkungen - publiziert worden. ${ }^{62}$ Die Aufgabe für die Wissenschaftler lautete: Was könnte die internationale Staatengemeinschaft tun, wenn sie die Summe von 50 Milliarden Dollar zur Verfügung hätte, um die Welt zu verbessern? ${ }^{63}$ Was sollte man mit hoher Priorität angehen? Die ausgewählten Fachleute formulierten 30 konkrete Vorschläge zur Bekämpfung globaler Probleme. Interessanterweise steht bei den Ökonomen die Medizin im Mittelpunkt der Thesen, während klassische politische Themen in den Hintergrund rücken. Die zweijährige Beratung durch eine erweiterte Gruppe von 50 international führenden Wirtschafts- und Finanzexperten stellte sicher, dass auf Basis der gegenwärtig verfügbaren Informationen entschieden werden konnte. Das Ergebnis ist in Bezug auf die zentrale Rolle der Heilkunde und die grundlegende Bedeutung des Gesundheitswesens eindrucksvoll: Unter den 30 wichtigsten Vorschlägen bezieht sich die Mehrheit auf Probleme der Medizin, allein 17 Punkte der Agenda fordern den Einsatz von Ressourcen zur Bekämpfung von Krankheiten bzw. zur Prävention. Besondere Aufmerksamkeit sollte laut den Kopenhagener Kosten-Nutzen-Rechnungen ${ }^{64}$ die Versorgung mit Mitteln zur Nahrungsergänzung und Eindämmung von Epidemien erhalten; der Kopenhagener Plan nennt für den Bereich Gesundheit und Medizin folgende Vorschläge: Mikronährstoffzusätze für Kinder (Vitamin A und Zink) sowie Nahrungsanreicherung, z.B. mit Eisengaben und Jodierung von Speisesalz, eine

Journal The Economist. Ein Kreis internationaler Experten, die globale Probleme aus interdisziplinärer Perspektive diskutierten, wurde konsultiert.

62 Pressemeldungen vom Juli 2008, siehe auch Lomborg (2006) und (2007).

63 Die genannte Summe erschien zunächst sehr hoch, aber im Zuge der umfangreichen Finanzpakete zur Rettung einzelner Banken oder Länder bzw. zur Konjunkturbelebung wurde sie in der jüngsten Vergangenheit erheblich relativiert und damit auch wieder möglicher gemacht. Es stellt sich also weiter die Frage: Warum ist für wichtige Probleme nicht genügend Kapital vorhanden?

64 Auf Kritik an einer ggf. allzu rational kalkulierten Menschlichkeit der Berechnungen der Kopenhagener Konzepte kann an dieser Stelle nicht vertiefend eingegangen werden. 
Ausweitung der Impfprogramme für Kinder, Entwurmung sowie Ernährungsprogramme an Schulen und Beratung auf kommunaler Ebene. Wichtige sinnvolle Programmpunkte beziehen sich auf Malaria- und Tuberkulosetherapien, preisgünstige Herzinfarktmedikamente für Entwicklungsländer, Bio-Sandfilter für die Reinigung von Wasser in Haushalten, Präventionskonzepte zur HIV-Bekämpfung, umfassende Sanitärprogramme zur Verringerung der Zahl offener Defäkationsstellen, eine Verbesserung chirurgischer Kapazitäten an Bezirkskrankenhäusern sowie Tabaksteuern zur Verringerung von Herzerkrankungen und Krebs.

Das internationale UN Millennium Project wie auch der KopenhagenKonsens unterstreichen und differenzieren zusammen mit einer Reihe weiterer Initiativen gleichermaßen die Zielsetzung, die zwei Generationen zuvor in der Universal Declaration of Human Rights ${ }^{65}$ formuliert wurde: Gesundheit ist wesentlicher Bestandteil der UNO-Ziele Frieden, Sicherheit und Wohlstand. Mit den Prinzipien Freiheit, Gleichheit, Gerechtigkeit und Menschenwürde sind in der Deklaration zudem auch für die Medizinethik zentrale Werte hervorgehoben worden.

Ein interessantes Projekt der jüngsten Zeit mit Bezug zum globalen Gesundheitswesen ist das Konzept eines »Health Impact Fund « von Thomas Pogge. ${ }^{66}$ Der Yale-Professor ist Schüler des Gerechtigkeitstheoretikers John Rawls. Mit der Offenlegung ungerechter sozialer Regeln auf globaler Ebene streben die philosophischen Vorschläge eine Verringerung der Weltarmut und eine Verbesserung der Medizin an. ${ }^{67}$ In Bezug auf das globale Gesundheitswesen gibt es etwa ökonomisch kreative Ideen für Anreizsysteme zur billigeren Verfügbarkeit dringend gebrauchter Medikamente: Mithilfe von Fondsausschüttungen sollen Probleme der Patentregelungen vermieden werden. Die Verbindung von philosophischer Grundlagenreflektion, anwendungsorientierter ökonomischer Gestaltung und politischer Überzeugungsarbeit ist an diesen Ansätzen positiv hervorzuheben.

65 Zur Declaration vgl. u.a. Annas (1998), Gruskin et al. (1998), Hutter/Kimmle (2008) und Frewer (2010).

66 Zum »Health Impact Fund« vgl. Hollis/Pogge (2008), Pogge (2009a) und (2009b); siehe auch www.healthimpactfund.org [10.12.2014].

67 Vgl. insbesondere Pogge (2001) und Pogge (2011); zur Verbindung von philosophischer und politischer Diskussion vgl. Nida-Rümelin et al. (2009). 


\section{SCHLUSSÜBERLEGUNGEN}

Artikel 1 der Allgemeinen Erklärung der Menschenrechte unterstreicht: »Alle Menschen sind frei und gleich geboren. Sie sind mit Vernunft und Gewissen begabt und sollen einander im Geist der Brüderlichkeit begegnen.« Verletzungen der Menschenrechte stehen hingegen leider weltweit an der Tagesordnung, auch über 70 Jahre nach Ende des Zweiten Weltkriegs sind Konflikte und Kriege weiterhin Realität. Eine »Kultur des Friedens« und globale Gerechtigkeit bleiben anzustrebende Ziele und vielfach Voraussetzung zur differenzierten Umsetzung des Menschenrechts auf Gesundheit in die Praxis. ${ }^{68}$ Signum einer globalisierten Welt sollte die Tatsache sein, dass niemand mehr »wegschauen« kann bei Hungersnöten, Flutkatastrophen oder Krankheitsausbreitung. Dies hatten eigentlich auch bereits die Epoche der Aufklärung und Protagonisten des 18. Jahrhunderts klar erkannt, aber die Globalisierung hat hier erst sukzessive den Gesichtskreis erweitert. »Das Recht der Menschen muß heilig gehalten werden, der herrschenden Gewalt mag es auch noch so große Aufopferung kosten. « ${ }^{69}$ Kants Konzept vom »ewigen Frieden« und Aufklärungsziele »vollkommener Gesundheit« in physischer wie psychischer und sozialer Hinsicht mögen utopisch klingen, fungieren jedoch als regulative Ideen; Medizin ist die Disziplin, mit der weltweit entscheidende Hilfe geleistet werden kann. Dies betrifft die allgemeine Entwicklung und humanitäre Kriseneinsätze ebenso wie die globale Vernetzung in Bezug auf Forschungsprojekte, Patente, Transfer von Gesundheitspersonal in westliche Länder ${ }^{70}$ oder strukturelle Fragen sozialer Regelungen internationaler Allokation (Verteilungsgerechtigkeit). Eine immer stärker kommerzialisierte Wahlfreiheit und überspannte Konzepte von Autonomie ${ }^{71}$ in reichen westlichen Gesundheitssystemen gehen auch mit Defiziten im Bereich nationaler Solidarität und der globalen

68 Vgl. u.a. Bialas et al. (1999) und Richter (2001).

69 Kant (1795) sowie Kant (1900-1955) und Höffe (2004).

70 Zu Fragen des »Brain drain« aus ärmeren Ländern vgl. etwa Shah (2010).

71 Die Frage, wie weit sich Gemeinschaft mit Autonomie verträgt - vgl. u.a. Miller (2000) - muss in mehrerlei Hinsicht auch global gestellt werden: Zu Herausforderungen einer »New Public Health« siehe etwa Beauchamp/Steinbock (1999) sowie exemplarisch für »Menschen ohne Papiere« Mylius et al. (2011). 
Weltgemeinschaft einher. Wenn darüber hinaus in Deutschland und in anderen Nationen die Einsparpotenziale bei Korruptionsverlusten, Medikamentenverschwendung und defensiver Medizin oder unnützen Therapien (futility) konsequent umgesetzt werden könnten, wären mehr Mittel für Mindeststandards und Universal Health Coverage vorhanden, im Sinne der Milleniumsziele wie auch des Kopenhagener Konsens oder künftiger Health Impact Fonds. Diese Instrumente und weiterentwickelte Modelle könnten wesentlich zu einer besseren Verwirklichung des Rechts auf Gesundheit beitragen. Manche Formen extremer oder überteuerter Spitzenmedizin in hochtechnologischen Bereichen müssen sich zudem in Bezug auf die globale Nachhaltigkeit kritisch hinterfragen lassen. ${ }^{72}$ Gerade die Verbindung der Humanmedizin mit Problemen von Gesellschaft, Ökonomie, Politik und Moraltheorie zeigt die komplexe Transdisziplinarität der behandelten Themen. Der Medizinethik ist die Einhaltung von Menschenrechten und Menschenwürde in allen Bereichen von Gesundheits- und Gemeinwesen ein zentrales Anliegen; durch den Einsatz von angemessener »Medizin - und Ethik - ohne Grenzen ${ }^{73}$ im Sinne reflektierter wie auch nachhaltiger Konzepte können wichtige Impulse für eine Umsetzung des globalen Menschenrechts auf Gesundheit gegeben werden. In Deutschland ist die Verabschiedung eines Gesetzes zu Patientenrechten ${ }^{74}$ ein weiterer Schritt. Sowohl der Schutz bei körperlicher Unversehrtheit (Beispiel Forschung) als auch die Durchsetzung ethischer Mindeststandards (Core Obligations) sind

72 Hier sei nur die Weltpremiere der »Transplantation von zwei Armen« genannt (2008), vgl. etwa Berndt (2008). Trotz Hochachtung vor der medizinischen Leistung könnte man kritische Fragen in Bezug auf die Allokation stellen, da der Patient jahrelang intensivste Betreuung braucht und ein Erfolg nicht sicher ist. Mit Blick auf globale Ressourcen könnte man pointiert formulieren, dass es sich in gewisser Hinsicht um die »Transplantation von 200 Armen« handelt. Zu einer instruktiven Übersicht von 500 lebensrettenden Eingriffen und ihrer KostenNutzen-Effektivität siehe auch die umfangreiche Studie von Tengs et al. (1995).

732016 findet der »Global Summit« der Nationalen Ethikräte zum Thema »Global Health, Global Ethics, Global Justice« in Berlin statt.

74 Vgl. insbesondere Emrich et al. (2014). 
erstrangige Desiderate eines menschenrechtlichen Ansatzes im Gesundheitswesen - auf nationaler wie globaler Ebene. ${ }^{75}$

\section{LITERATUR}

Advisory Committee on Human Radiation Experiments (1996): The Human Radiation Experiments: Final Report of the President's Advisory Committee, New York: Oxford University Press.

Andreopoulos, George J. (2000): »Declarations and Covenants of Human Rights and International Codes of Research Ethics«, in: Levine et al. (2000), 181-203.

Annas, George J. (1998): »Human Rights and Health. The Universal Declaration of Human Rights at 50«, in: New England Journal of Medicine 339 (1998), 1778-1781.

Bahnsen, Ulrich (2001): »Klonen. Der Wahn des Doktor Antinori. Forscher wollen Menschen klonen. Dabei ignorieren sie ethische Bedenken und biologische Risiken«, Online: www.zeit.de/2001/12/ 200112_klonen. xml [28.08.2014].

Beauchamp, Dan E./Steinbock, Bonnie (Eds.) (1999): New ethics for the public's health, New York, Oxford: Oxford University Press.

Beauchamp, Tom L./Childress, James F. (2009): Principles of biomedical ethics. 6. Auflage. New York, Oxford: Oxford University Press.

Beerheide, R./Richter-Kuhlmann, E. (2015): „Gesetz zur Palliativ- und Hospizversorgung. Umsetzung ist entscheidend«, in: Deutsches Ärzteblatt 112, 46 (2015), A 1920-1922.

Bernard, Claude (1961): Einführung in das Studium der experimentellen Medizin. Ins Deutsche übertragen von P. Szendrö und biographisch eingeführt und kommentiert von K. E. Rothschuh. Leipzig: Barth.

Berndt, Christina (2008): »Ganze Arme transplantiert. >Jetzt bin ich bereitく. Der Patient hatte beide Arme bei einem Unfall verloren. Dann fasste der 54-Jährige den Entschluss, sich die Gliedmaßen eines Toten annähen zu lassen. Eine medizinische Weltpremiere«, Online: www.sueddeutsche.

75 Die Professur für Ethik in der Medizin (FAU) verfolgt seit 2012 in Kooperation mit der Ethik-Abteilung der World Health Organisation (WHO/Genf) das Projekt »Global Health Ethics« zur Illustration internationaler Problemfelder (in Forschung, Lehre und bei einer Ausstellung). 
de/wissen/ganze-arme-transplantiert-jetzt-bin-ich-bereit-1.595487 [28.08.2014].

Bialas, Volker/Häßler, Hans-Jürgen/Woit, Ernst (Hg.) (1999): Die Kultur des Friedens. Weltordnungsstrukturen und Friedensgestaltung, Würzburg: Königshausen \& Neumann.

Bockhart, Max (1883): »Beitrag zur Ätiologie und Pathologie des Harnröhrentrippers«, in: Vierteljahresschrift für Dermatologie und Syphilis 10 (1883), 3-18.

Bonah, Christian/Lepicard, Étienne/Roelcke, Volker (Hg.) (2003): La médecine expérimentale au tribunal. Implications éthiques de quelques procès médicaux du XXe siècle européen, Paris: éditions des archives contemporaines.

CIB/DPA (2010): »Hwang Woo Suk. Gericht verurteilt Klonfälscher zu Bewährungsstrafe«, Online: www.spiegel.de/wissenschaft/mensch/ hwang-woo-suk-gericht-verurteilt-klonfaelscher-zu-bewaehrungsstrafe -a-735139.html [28.08.2014].

Clapham, Andrew (2006): Human Rights Obligations of Non-State Actors, New York: Oxford University Press.

Council for International Organizations of Medical Sciences (CIOMS), in collaboration with the World Health Organization (1993): International Ethical Guidelines for Biomedical Research Involving Human Subjects, Geneva: CIOMS.

Council for International Organizations of Medical Sciences (CIOMS), in collaboration with the World Health Organization (2002): International Ethical Guidelines for Biomedical Research Involving Human Subjects, revised edition, Online: www.cioms.ch [10.12.2014].

Council for International Organizations of Medical Sciences (CIOMS), in collaboration with the World Health Organization (2009): International Ethical Guidelines for Epidemiological Studies, Geneva: CIOMS.

Deichgräber, Karl (1983): Der hippokratische Eid. 4. Auflage, Stuttgart: Kröner.

Diller, Hans (1994): Hippokrates. Ausgewählte Schriften, Stuttgart: Reclam.

Eckart, Wolfgang U. (2013): Geschichte der Medizin. Fakten, Konzepte, Haltungen. 7. Auflage, Berlin, Heidelberg: Springer.

Edelstein, Ludwig (1969): Der Hippokratische Eid, Zürich, Stuttgart: Artemis. 
Edelstein, Wolfgang/Nunner-Winkler, Gertrud (Hg.) (2000): Moral im sozialen Kontext, Frankfurt/M.: Suhrkamp.

Eichinger, Tobias (2013): Jenseits der Therapie. Philosophie und Ethik wunscherfüllender Medizin, Bielefeld: transcript.

Elkeles, Barbara (1996): Der moralische Diskurs über das medizinische Menschenexperiment im 19. Jahrhundert. Medizinethik, Bd. 7, Stuttgart: G. Fischer.

Emanuel, Ezekiel J./Emanuel, Linda L. (1992): »Four Models of the Physician-Patient Relationship«, in: Journal of the American Medical Association 267, 16 (1992), 2221-2226.

Emrich, Inken/Fröhlich-Güzelsoy, Leyla/Frewer, Andreas (Hg.) (2014): Ethik in der Medizin aus Patientensicht. Perspektivwechsel im Gesundheitswesen. Klinische Ethik, Bd. 4, Frankfurt/M. u.a.: Lang.

Erices, Rainer/Gumz, Antje/Frewer, Andreas (2014): Westliche Humanexperimente in der DDR und die Deklaration von Helsinki. Neue Forschungsergebnisse zur Ethik, in: Jahrbuch Medizin-Ethik 27 (2014), 87-98.

Eschebach, Insa/Ley, Astrid (Hg.) (2012): Geschlecht und »Rasse« in der NS-Medizin, Berlin: Metropol-Verlag.

Frewer, Andreas (2000): Medizin und Moral in Weimarer Republik und Nationalsozialismus, Frankfurt/M., New York: Campus.

Frewer, Andreas (2009): »Menschenrechte, Medizin und Moral. Zur Einführung«, in: Frewer et al. (2009b), 11-22.

Frewer, Andreas (2010): »Human rights from the Nuremberg Doctors Trial to the Geneva Declaration. Persons and institutions in medical ethics and history«, in: Medicine, Health Care and Philosophy 13 (2010), 259-268.

Frewer, Andreas (2013): »Menschenrecht auf Gesundheit. Die Patientenautonomie und das Gemeinwohl«, in: Möseneder (2013), 25-48.

Frewer, Andreas/Bruns, Florian/Rascher, Wolfgang (Hg.) (2011): Gesundheit, Empathie und Ökonomie. Kostbare Werte in der Medizin, Jahrbuch Ethik in der Klinik (JEK), Bd. 4, Würzburg: Königshausen \& Neumann.

Frewer, Andreas/Fahr, Uwe/Rascher, Wolfgang (Hg.) (2009a): Patientenverfügung und Ethik. Beiträge zur guten klinischen Praxis, Jahrbuch Ethik in der Klinik (JEK), Bd. 2, Würzburg: Königshausen \& Neumann. 
Frewer, Andreas/Kolb, Stephan/Krása, Kerstin (Hg.) (2009b): Medizin, Ethik und Menschenrechte. Geschichte - Grundlagen - Praxis. Medizin und Menschenrechte, Bd. 1, Göttingen: V \& R unipress.

Frewer, Andreas/Neumann, Josef N. (Hg.) (2001): Medizingeschichte und Medizinethik. Kontroversen und Begründungsansätze 1900-1950, Frankfurt/M., New York: Campus.

Frewer, Andreas/Oppitz, Ulrich-Dieter et al. (Hg.) (1999): Medizinverbrechen vor Gericht. Das Urteil im Nürnberger Ärzteprozeß gegen Karl Brandt und andere sowie aus dem Prozeß gegen Generalfeldmarschall Erhard Milch, Erlangen, Jena: Palm \& Enke.

Frewer, Andreas/Schmidt, Ulf (Hg.) (2007): Standards der Forschung. Historische Entwicklung und ethische Grundlagen klinischer Studien, Frankfurt/M. u.a.: Lang.

Frewer, Andreas/Schmidt, Ulf (Hg.) (2014): Forschung als Herausforderung für Ethik und Menschenrechte. 50 Jahre Deklaration von Helsinki (1964-2014), Jahrbuch Medizin-Ethik 27 (2014), Köln: Deutscher Ärzte-Verlag.

Frewer, Andreas/Siedbürger, Günther (Hg.) (2004): Zwangsarbeit und Medizin im Nationalsozialismus. Einsatz und Behandlung von »Ausländern« im Gesundheitswesen, Frankfurt/M., New York: Campus.

Fritsche, Paul (1987): »Die Menschenrechte angesichts der Entwicklung von Wissenschaft und Technik aus ärztlicher Sicht«, in: Von Bülöw/ Fritsche (1987), 23-45.

Genest, Andrea (Hg.) (2015): Damit die Welt es erfährt ... Illegale Dokumente polnischer Häftlinge aus dem Konzentrationslager Ravensbrück. Mit einem Vorwort von Wanda Kiedrzyńska. Aus dem Polnischen übersetzt von Inge Gerlinghoff und Barbara Lubos-Kroll, Berlin: Metropol-Verlag.

Grover, Anand (2013): Report of the Special Rapporteur on the right of everyone to the enjoyment of the highest attainable standard of physical and mental health, Geneva: United Nations.

Gruskin, Sofia/Grodin, Michael A./Annas, George J./Marks, Stephen P. (Eds.) (2005): Perspectives on Health and Human Rights, New York, London: Routledge.

Gruskin, Sofia/Mann, Jonathan/Annas, George J./Grodin, Michael A./ Rawson, Brian/Ruff, Tilman/Sidel, Victor W./Ayotte, Barbara/Iacopino, Vincent/Rubenstein, Len/Sirkin, Susannah [Writing Group for the 
Consortium for Health and Human Rights] (1998): »Health and Human Rights: A Call to Action on the 50th Anniversary of the Universal Declaration of Human Rights«, in: Journal of the American Medical Association 280 (1998), 462-464.

Habermas, Jürgen (2001): Die Zukunft der menschlichen Natur. Auf dem Weg zu einer liberalen Eugenik?, Frankfurt/M.: Suhrkamp.

Harrington, John/Stuttaford, Maria (Eds.) (2010): Global health and human rights. Legal and philosophical perspectives. London u.a.: Routledge.

Höffe, Otfried (Hg.) (2004): Immanuel Kant, zum ewigen Frieden, 2. Auflage, Berlin: Akademie-Verlag.

Hollis, Aidan/Pogge, Thomas (2008): The Health Impact Fund. Making new medicines accessible for all. Incentives for Global Health, New Haven, Oslo: Incentives for Global Health (IGH).

Hutter, Franz-Josef/Kimmle, Carsten (Hg.) (2008): 60 Jahre Allgemeine Erklärung der Menschenrechte. Das uneingelöste Versprechen, Karlsruhe: Loeper Literaturverlag.

Joerden, Jan/Hilgendorf, Eric/Petrillo, Natalia./Thiele, Felix (Hg.) (2011): Menschenwürde und moderne Medizintechnik, Baden-Baden: Nomos.

Jones, Jarhes H. (1981): Bad blood. The Tuskegee Syphilis Experiment, New York: Free Press.

Jung, Eberhard (1982): Das Recht auf Gesundheit. Versuch einer Grundlegung des Gesundheitsrechts der Bundesrepublik Deutschland, München: Beck.

Kant, Immanuel (1795): Zum ewigen Frieden. Ein philosophischer Entwurf. Berlin, Leipzig: Königlich Preußische/Deutsche Akademie der Wissenschaften Berlin.

Kant, Immanuel (1900-1955): Gesammelte Schriften in 23 Bänden, Berlin, Leipzig: Königlich Preußische/Deutsche Akademie der Wissenschaften Berlin.

Kettner, Matthias (Hg.) (2009): Wunscherfüllende Medizin. Ärztliche Behandlung im Dienst von Selbstverwirklichung und Lebensplanung, Kultur der Medizin, Bd. 27, Frankfurt/M., New York: Campus.

Kettner, Matthias/Kraska, Matthias (2009): »Kompensation von ArztPatient-Asymmetrien im Rahmen einer Theorie kommunikativen Handelns«, in: Vollmann et al. (2009), 243-259. 
Klein, Eckart/Menke, Christoph (Hg.) (2004): Menschenrechte und Bioethik, Menschenrechtszentrum der Universität Potsdam, Bd. 21, Berlin: Berliner Wissenschafts-Verlag.

Lehner, Anna M./Frewer, Andreas (2014): »Forschung gegen Menschenrechte und die Helsinki-Deklaration? Zu Gehirnstudien und Gefängnisexperimenten in den USA der Nachkriegszeit«, in: Jahrbuch Medizin-Ethik 27 (2014), 35-52.

Leibniz, Gottfried Wilhelm (1976): Directiones ad rem medicam pertinentes. Ein Manuskript G. W. Leibnizens aus dem Jahr 1671/72. Übersetzt und kommentiert von Fritz Hartmann und Matthias Krüger, Studia Leibnitiana 8 (1976), 40-68.

Ley, Astrid/Ruisinger, Maria (Hg.) (2001): Gewissenlos - gewissenhaft. Menschenversuche im Konzentrationslager, Erlangen-Nürnberg: Specht.

Lomborg, Björn (Ed.) (2006): How to Spend \$ 50 Billion to Make the World a Better Place, Cambridge: Cambridge University Press.

Lomborg, Björn (Ed.) (2007): Solutions for the World's Biggest Problems, Costs and Benefits. Cambridge: Cambridge University Press.

Macklin, Ruth (2004): Double Standards in Medical Research in Developing Countries, Cambridge: Cambridge University Press.

Macklin, Ruth (2010): »Intertwining Biomedical Research and Public Health in HIV Preventive Microbicide Research«, in: Public Health Ethics 3, 3 (2010), 199-209.

Maio, Giovanni (2012): Mittelpunkt Mensch: Ethik in der Medizin. Ein Lehrbuch, Stuttgart:

Manok, Andreas (2012): Body integrity identity disorder. Die Zulässigkeit von Amputationen gesunder Gliedmaßen aus rechtlicher Sicht, Leipziger juristische Studien, Bd. 8. Leipzig: Leipziger Universitäts-Verlag.

Marks, Stephen P. (Ed.) (2006): Health and Human Rights: Basic International Documents. 2nd ed. Cambridge, Massachusetts: FrancoisXavier Bagnoud Center for Health and Human Rights, Harvard School of Public Health.

Miller, Joan G. (2000): »Verträgt sich Gemeinschaft mit Autonomie? Kulturelle Ideale und empirische Wirklichkeiten«, in: Edelstein/NunnerWinkler (2000), 337-362.

Möseneder, Karl (Hg.) (2013): Menschenrechte. Universitätstage, Erlangen: FAU University Press. 
Moll, Albert (1902): Ärztliche Ethik. Die Pflichten des Arztes in allen Beziehungen seiner Thätigkeit, Stuttgart: Enke.

Moreno, Jonathan D. (2000): Undue risk. Secret state experiments on humans, London, New York: Routledge.

Mylius, Maren/Bornschlegl, Wiebke/Frewer, Andreas (Hg.) (2011): Medizin für »Menschen ohne Papiere«. Menschenrechte und Ethik in der Praxis des Gesundheitssystems, Medizin und Menschenrechte, Bd. 5, Göttingen: V \& R unipress.

Nida-Rümelin, Julian/Thierse, Wolfgang/Wieczorek-Zeul, Heidemarie/ Weißkirchen, Gert (Hg.) (2009): Thomas Pogge. Gerechtigkeit in der Einen Welt, Kultur in der Diskussion, Bd. 15, Essen: Klartext Verlag.

Participants in the 2001 Conference on Ethical Aspects of Research in Developing Countries (2004): »Moral Standards for Research in Developing Countries: From >Reasonable Availability « to >Fair Benefits««, in: Hastings Center Report 34 (2004), 17-27.

Plomer, Aurora (2004): »In Defence of Helsinki and Human Rights«, in: South African Journal of Bioethics and Law 5, 2 (2004), 83-86.

Pogge, Thomas (2001): Global justice, Malden/Massachusetts: Blackwell.

Pogge, Thomas (2009a): »Warum die Menschenrechte die Einrichtung des Health Impact Fund verlangen«, in: Zeitschrift für Menschenrechte 2 (2009), 124-161.

Pogge, Thomas (2009b): Der Health Impact Fund. Wie Pharmaforschung wirklich allen zugute kommen kann. Gerechte Gesundheit: Das Portal zur Verteilungsdebatte, Online: www.gerechte-gesundheit.de/debatte/ autorenbeitraege/detail/ab-eintrag/ 1/6.html [12.07.2015].

Pogge, Thomas (2011): Weltarmut und Menschenrechte. Kosmopolitische Verantwortung und Reformen. Übers. von Anna Wehofsits. Berlin: de Gruyter.

Quante, Michael (2010): Menschenwürde und personale Autonomie. Demokratische Werte im Kontext der Lebenswissenschaften, Hamburg: Meiner.

Ramm, Thilo (2008): „Sozialstaatsprinzip und Recht auf Gesundheit. Deutsches Gesundheitsrecht am Scheideweg?«, in: Vierteljahresschrift für Sozialrecht 4, 203-219.

Reich, Warren T. (Ed.) (1995): Encyclopedia of bioethics, Vols. 1-5, New York: Macmillan Reference USA, Thomson Gale. 
Richter, Horst-Eberhard (Hg.) (2001): Kultur des Friedens. Deutsche Sektion der Internationalen Ärzte für die Verhütung des Atomkrieges Ärzte in sozialer Verantwortung e.V., Giessen: Psychosozial.

Richter-Kuhlmann, E. (2015): »Suizidbeihilfe. Verbot bei Geschäftsmäßigkeit«, in: Deutsches Ärzteblatt 112, 46 (2015), A 1918.

Roelcke, Volker (2012): »Fortschritt ohne Rücksicht. Menschen als Versuchskaninchen bei den Sulfonamid-Experimenten im Konzentrationslager Ravensbrück«, in: Eschebach/Ley (2012), 101-114.

Roelcke, Volker/Maio, Giovanni (Hg.) (2004): Twentieth Century Ethics of Human Subject Re-search. Historical Perspectives on Values, Practices, and Regulations, Stuttgart: Steiner.

Sabisch, Katja (2007): Das Weib als Versuchsperson. Medizinische Menschenexperimente im 19. Jahrhundert am Beispiel der Syphilisforschung, Bielefeld: transcript.

Schäfer, Daniel/Frewer, Andreas/Schockenhoff, Eberhard/Wetzstein, Verena (Hg.) (2008): Gesundheitskonzepte im Wandel. Geschichte, Ethik und Gesellschaft, Stuttgart: Steiner Verlag.

Schäfer, Daniel/Müller-Busch, Christoph/Frewer, Andreas (Hg.) (2012): Perspektiven zum Sterben. Auf dem Weg zu einer Ars moriendi nova?, Ars moriendi nova, Bd. 2, Stuttgart: Steiner.

Schimmelbusch, Curt T. (1888): »Ueber die Ursachen der Furunkel«, in: Archiv für Ohrenheilkunde 27 (1888), 252-264.

Schmidt, Ulf (2006): Justice at Nuremberg. Leo Alexander and the Nazi doctors' trial, Houndsmills u.a.: Palgrave Macmillan.

Schmidt, Ulf/Frewer, Andreas (Eds.) (2007): History and Theory of Human Experimentation. The Declaration of Helsinki and Modern Medical Ethics, Stuttgart: Steiner.

Schmidt, Ulf/Frewer, Andreas (2014): »The Declaration of Helsinki as a Landmark for Research Ethics. Protecting Human Participants in Modern Medicine«, in: Wiesing et al. (2014), 56-57.

Schubert, Charlotte (2005): Der hippokratische Eid. Medizin und Ethik von der Antike bis heute, Darmstadt: Wissenschaftliche Buchgesellschaft.

Seelmann, Kurt (2008): »Recht auf Gesundheit? Über den Wandel juristischer Perspektiven auf das Gesundheitswesen«, in: Schäfer et al. (2008), 177-187.

Shah, Rebecca S. (Ed.) (2010): The international migration of health workers: ethics, rights and justice, Basingstoke: Palgrave Macmillan. 
Stiftung kreuznacher diakonie, Der Vorstand/Ethikausschuss (2011): Grundsätze zur Behandlung von Zeugen Jehovas. Diakonisch-ethische Positionen. Bad Kreuznach.

Stirn, Aglaja/Thiel, Aylin/Oddo, Silvia (Hg.) (2010): Body Integrity Identity Disorder (BIID). Störungsbild, Diagnostik, Therapieansätze, Weinheim: Beltz.

Tag, Brigitte (2000): Der Körperverletzungstatbestand im Spannungsfeld zwischen Patientenautonomie und Lex artis. Eine arztstrafrechtliche Untersuchung, Berlin u.a.: Springer.

Tengs, Tarnmy O./Adams, Miriam E./Pliskin, Joseph S./Safran, Dana Gelb/Siegel, Joanna E./Weinstein, Milton C./Graham, John D. (1995): »Five-Hundred Life-Saving Interventions and Their Cost-Effectiveness «, in: Risk Analysis 15, 3 (1995), 369-390.

Toebes, Brigit C. A. (1999): The Right to Health as a Human Right in International Law, Antwerpen u.a.: Intersentia - Hart.

Toebes, Brigit C. A. (Ed.) (2014): The Right to Health. A Multi-Country Study of Law, Policy and Practice. The Hague: T.M.C. Asser Press.

Toellner, Richard (Hg.) (1992): Illustrierte Geschichte der Medizin, Sonderausgabe, Erlangen: Andreas Verlag.

Tröhler, Ulrich/Reiter-Theil, Stella (Hg.) (1997): Ethik und Medizin 19471997. Was leistet die Kodifizierung von Ethik?, Göttingen: Wallstein.

UNESCO (2005): Universal declaration on bioethics and human rights (adopted by UNESCO’s General Conference on 19.11.2005), Online: www.unesco.org/new/en/social-and-human-sciences/themes/bioethics/ bioethics-and-human-rights/Paris: UNESCO [06.12.2008].

UNHCR (2008): The right to health. Human rights fact sheet 31, Office of the United Nations High Commissioner for Human Rights, Geneva: UNHCR.

Van der Walt, Sibylle (2007): „Einleitung: Der neue Begründungsbedarf des Menschenrechts auf körperliche Unversehrtheit«, in: Van der Walt/ Menke (2007), 7-24.

Van der Walt, Sibylle/Menke, Christoph (Hg.) (2007): Die Unversehrtheit des Körpers. Theorie und Geschichte eines elementaren Menschenrechts, Frankfurt/M., New York: Campus.

Von Bülow, Detlev (1987): »Die Menschenrechte angesichts der Entwicklung von Wissenschaft und Technik - Rechtsfragen der Medizin«, in: von Bülöw/Fritsche (1987), 1-16. 
Von Bülow, Detlev/Fritsche, Paul (1987): Menschenrechte in der modernen Medizin. Rechtsstaat in der Bewährung, Bd. 21, Heidelberg: C.F. Müller Juristischer Verlag.

Wachtturm Bibel- und Traktatgesellschaft (2000): »Wie sind Jehovas Zeugen angesichts biblischer Gebote über die passende Verwendung von Blut zu medizinischen Verfahren eingestellt, bei denen Eigenblut verwendet wird? Fragen von Lesern«, in: Der Wachtturm, 15.10.2000, 3031.

Weindling, Paul (2004): Nazi medicine and the Nuremberg Trials. From medical war crimes to informed consent, Houndmills u.a.: Palgrave Macmillan.

Weindling, Paul (2012): „Die Opfer von Humanexperimenten im Nationalsozialismus. Ergebnisse eines Forschungsprojekts«, in: Eschebach/Ley (2012), 81-100.

Wiesing, Urban/Parsa-Parsi, Ramin/Kloiber, Otmar (Eds.) (2014): The World Medical Association Declaration of Helsinki. 1964-2014. 50 years of evolution of medical research ethics, Köln: Deutscher Ärzte-Verlag.

Zude, Heiko Ulrich (2010): Paternalismus. Fallstudien zur Genese des Begriffs, Freiburg i.Br.: Alber. 


\section{Die Konkretisierung von Kernbereichen des Menschenrechts auf Gesundheit}

Internationale Debatten zu »Minimum Core Obligations«

AMREI MÜLLER

\section{EINLEITUNG ${ }^{1}$}

Dieser Aufsatz stellt die Frage nach dem Kernbereich oder Kerngehalt des Menschenrechts auf Gesundheit und den zugehörigen Kernverpflichtungen, die in jedem Vertragsstaat des Internationalen Pakts über wirtschaftliche, soziale und kulturelle Rechte (IPwskR, kurz Sozialpakt) ${ }^{2}$ vorrangig umgesetzt werden müssen. Obwohl der Kernbereichsansatz zum Recht auf Gesundheit in der Menschenrechtsliteratur bereits viel diskutiert und analysiert wurde, ${ }^{3}$ bleiben zahlreiche offene Fragen. Unter diesen sind Fragen nach dem konkreten Inhalt eines Kernbereichs des Rechts auf Gesundheit und ob dieser auf internationaler oder nationaler Ebene festgelegt werden sollte. Eine Beantwortung dieser Fragen ist notwendig, um mindestens den

1 Die Autorin möchte dem Editionsteam des Bandes für die hilfreichen Kommentare und sprachliche Verbesserungen herzlich danken. Teile dieses Beitrags wurden in englischer Sprache in Müller (2013), Kap. 4 veröffentlicht; der vorliegende Artikel wurde überarbeitet und aktualisiert.

2 Internationaler Pakt über wirtschaftliche, soziale und kulturelle Rechte (IpwskR), 933 UNTS 3, 16. Dezember 1966.

3 Unter vielen siehe z.B. Bilchitz (2007) sowie die Beiträge in Chapman/Russell (2002). 
Kerngehalt des Rechts auf Gesundheit aller Menschen, die in verschiedensten Umständen und Ländern leben, effektiv umzusetzen, einschließlich der Überwachung durch nationale und internationale Gerichte oder durch andere außergerichtliche Mechanismen. In diesem Kontext konzentriert sich der Aufsatz besonders auf die Frage nach dem Zusammenspiel von international definierten materiellen und verfahrensbezogenen Kernverpflichtungen aus dem Recht auf Gesundheit sowie deren Beitrag zur Konkretisierung des Rechts auf Gesundheit auf nationaler Ebene und seiner effektiven Umsetzung.

Der Aufsatz beginnt mit einer Erklärung, warum es wichtig bleibt, eine klare Konzeption des Kernbereichsansatzes zu erarbeiten. In diesem Zusammenhang wird der Grundgedanke, der hinter dem Kerngehaltsansatz steht, in Erinnerung gerufen (Teil 2). Als nächster Schritt werden in Teil 3 die Problembereiche der vorherrschenden Konzeption des Kernbereichsansatzes diskutiert. Der Fokus liegt hierbei auf der (unklaren) Konzeption des Kerngehalts des Rechts auf Gesundheit wie er vom Ausschuss für wirtschaftliche, soziale und kulturelle Rechte der Vereinten Nationen (im Folgenden »UN-Ausschuss zum Sozialpakt « oder »UN-Sozialausschuss«) dargestellt wird. Teil 4 entwickelt eine alternative Konzeption des Kernbereichsansatzes basierend auf der Rechtsprechung nationaler und internationaler Gerichte sowie den Erklärungen des UN-Sozialausschusses und anderer internationaler bzw. regionaler Organisationen. Diese Alternative hebt zwei Aspekte besonders hervor: erstens die Frage nach einem sinnvollen Verhältnis zwischen einem generellen, universell definierten und einem konkreteren, national definierten materiellen Kernrechts auf Gesundheit; und zweitens die damit zusammenhängende Frage nach der Rolle, welche international definierte materielle und verfahrensbezogene Verpflichtungen bei der Konkretisierung des Kernbereiches auf nationaler Ebene spielen. In den Schlussbemerkungen (Teil 5) werden die wichtigsten Punkte kurz zusammengefasst. 


\section{KeRnBEREICHE DES RECHTS AUf Gesundheit: GRUNDGEDANKE UND POTENZIAL}

$\mathrm{Zu}$ Beginn soll der Grundgedanke des Kernbereichsansatzes (minimum core approach) in Erinnerung gerufen werden. Es geht also um die Frage, warum der UN-Fachausschuss zum Sozialpakt diesen Ansatz überhaupt gewählt hat. Der Hauptgrund war, den Begriff der »schrittweisen Umsetzung « wirtschaftlicher, sozialer und kultureller Rechte (WSK-Rechte), wie er sich in Artikel 2 (1) IPwskR findet, ${ }^{4}$ zu konkretisieren und damit die Beurteilungsmöglichkeiten der Schritte und Maßnahmen, die Staaten zur schrittweisen Umsetzung des Rechts auf Gesundheit und anderer WSK-Rechte ergreifen, zu verbessern. Der Kerngehaltsansatz wurde auch entwickelt, um starken Tendenzen innerhalb der Rechtslehre und den Argumenten einiger Staaten entgegenzuwirken, ${ }^{5}$ dass WSK-Rechte keine konkreten Verpflichtungen beinhalten, da sie nach dem Wortlaut des IPwskR nur »schrittweise« umgesetzt werden müssen und für ihre Umsetzung beträchtliche Ressourcen benötigt werden. ${ }^{6}$

In seiner Allgemeinen Bemerkung Nr. 3 hat der UN-Sozialausschuss die Grundzüge des Kernbereichsansatzes skizziert. Der Ausschuss verkündete:

»a minimum core obligation to ensure the satisfaction of, at the very least, minimum essential levels of each of the rights is incumbent upon every State party. Thus, for example, a State party in which any significant number of individuals is deprived of essential foodstuffs, of essential primary health care, of basic shelter or housing, or of the most basic form of education is, prima facie, failing to discharge its obligations under the Covenant. If the Covenant were to be read in such a way as not

4 Artikel 2 (1) IPwskR: »Jeder Vertragsstaat verpflichtet sich, einzeln und durch internationale Hilfe und Zusammenarbeit, insbesondere wirtschaftlicher und technischer Art, unter Ausschöpfung aller seiner Möglichkeiten Maßnahmen zu treffen, um nach und nach mit allen geeigneten Mitteln, vor allem durch gesetzgeberische Maßnahmen, die volle Verwirklichung der in diesem Pakt anerkannten Rechte zu erreichen.«

5 Für eine Zusammenfassung der Debatte siehe Eide et al. (2001), insbesondere Kapitel 1-3.

6 Siehe E/1991/23, 14. Dezember 1990, para. 9. 
to establish such a minimum core obligation, it would be largely deprived of its raison d'être. ${ }^{7}$

Wie Matthew Craven bemerkt, ist der Grundgedanke hinter dem Kerngehaltsansatz somit ein relativ simpler: Die Festlegung von quantitativen und qualitativen Mindeststandards zu jedem WSK-Recht, welcher unter allen Umständen für alle Personen, die sich unter der Herrschaftsgewalt eines Vertragsstaates befinden, als Angelegenheit höchster Priorität gewährleistet werden muss. ${ }^{8}$ Der Wesensgehalt eines jeden WSK-Rechts ist mit grundlegenden menschlichen Interessen verbunden - häufig mit überlebenswichtigen Bedürfnissen. ${ }^{9}$ Um einige Beispiele zu nennen, hat der UN-Sozialausschuss festgestellt, dass der Kernbereich des Rechts auf angemessene Nahrung das Recht auf Zugang zu einer Mindestmenge an Grundnahrungsmitteln umfasst, die ausreichend ist, um Hunger zu vermeiden; ${ }^{10}$ das Recht auf Zugang zu grundlegender Bildung, das ein Minimum an Teilhabe im gesellschaftlichen Leben ermöglicht, ist der Kerngehalt des Rechts auf Bildung; ${ }^{11}$ und das Recht auf Zugang zu einem primären Gesundheitsversorgungssystem, welches die elementaren gesundheitlichen Probleme der Bevölkerung angehen und bekämpfen kann, stellt den Kerngehalt des Rechts auf Gesundheit dar. ${ }^{12}$ Sobald ein Vertragsstaat den Mindeststandard umgesetzt hat, müssen die Verpflichtungen, die über den Kernbereich hin-

7 E/1991/23, 14. Dezember 1990, para. 10.

8 Siehe E/1991/23, 14. Dezember 1990, para. 10; E/C.12/2002/11, 20. Januar 2003, para. 6; E/C.12/GC/19, 4. Februar 2008, para. 60; E/C.12/2000/4, 11. August 2000, para. 47 und E/CN.12/1999/5, 12. Mai 1999, para. 17.

9 In diesem Kontext ist auf die philosophischen Argumente von David Bilchitz und Thomas Pogge hinzuweisen, denn sie verknüpfen Kerngehalte der WSKRechte mit dem fundamentalen menschlichen Überlebensinteresse und dem Interesse an minimalem Wohlergehen, deren Erfüllung eine grundlegende Voraussetzung dafür ist, dass Menschen positive Lebenserfahrungen haben und ihre Lebenszwecke erfüllen (Bilchitz) oder ein aufblühendes Leben (»flourishing life«) genießen können (Pogge); vgl. Bilchitz (2007) und Pogge (2002).

10 Siehe E/1991/23, 14. Dezember 1990, para. 10, E/C.12/2000/4, 11. August 2000, para. 12 und Künnemann (2002), 171.

11 E/C.12/1999/10, 8. Dezember 1999, para. 57.

12 E/C.12/2000/4, 11. August 2000, paras. 43-44. 
ausgehen, zielstrebig und sobald als möglich schrittweise implementiert werden, unter maximaler Verwendung der bereitstehenden Ressourcen. ${ }^{13}$

Der Kernbereichsansatz verspricht nicht nur, dass »wirtschaftlichen und sozialen Rechten Bestimmtheit und Klarheit verliehen wird « ${ }^{14}$ - zumindest zu einem gewissen Grad -, sondern auch, dass vorhandene Mittel und fachliche Kapazitäten dort verwendet werden, wo man sie am meisten benötigt, sodass Personen, die nichts oder sehr wenig besitzen, vornehmlich davon profitieren - in den Worten des UN-Fachausschusses: »benachteiligte und marginalisierte Bevölkerungsgruppen und Einzelpersonen $«{ }^{15}$ Der Ausschuss nutzt den Kernbereichsansatz auch, um individuelles und kollektives staatliches Handeln außerhalb der eigenen Staatsgrenzen zu bewerten, zum Beispiel im Kontext globaler Handels- und Entwicklungspolitik, ${ }^{16}$ sowie der Sicherheitspolitik (Nahrungsmittelembargos). ${ }^{17}$ In seiner »Erklärung zu Armut und dem IPwskR « hat der Ausschuss festgestellt, dass sich aus Kernrechten »nationale Verpflichtungen aller Staaten ergeben, sowie internationale Verantwortlichkeiten entwickelter Staaten und all derer, die ssich in einer Position finden, Hilfe zu leisten « ${ }^{18}{ }^{18}$ Somit verspricht der Kernbereichsansatz auch, nationale Verpflichtungen und internationale Verantwortlichkeiten für die Umsetzung des Rechts auf Gesundheit und anderer WSK-Rechte voneinander abzugrenzen und zu koordinieren. Das ist besonders wichtig für die Implementierung der WSK-Rechte (einschließlich ihrer Kernbereiche) in ressourcenarmen Staaten, die auf (verlässliche) Hilfe von außen angewiesen sind.

\section{Im Einklang mit Artikel 2 (1) ICESCR.}

14 Bueren (1999), 57 [eigene Übersetzung] und Wesson (2004), 299-300.

15 E/C.12/GC/19, 4. Februar 2008, para. 59 (e); E/C.12/2002/11, 20. Januar 2003, paras. 37 (b) und (f); E/C.12/2000/4, 11. August 2000, paras. 43 (a) und (f) sowie E/CN.12/1999/5, 12. Mai 1999, para. 28.

16 Vgl. z.B. E/C.12/GC/19, 4. Februar 2008, para. 61; E/C.12/GC/18, 6. Februar 2006, para. 30; E/C.12/2002/11, 20. Januar 2003, para. 38; E/C.12/2000/4, 11. August 2000, para. 45 und Statement on Poverty and the ICESCR, E/C.12/2001/10, 10. Mai 2001, paras. 16-17.

17 Vgl. z.B. E/C.12/1997/8, 12. Dezember 1997, para. 7; E/C.12/2002/11, 20. Januar 2003, para. 32 und E/CN.12/1999/5, 12. Mai 1999, para. 37.

18 E/C.12/2001/10, 10. Mai 2001, para. 16 [eigene Übersetzung]. 
Schließlich ist der Kernbereichsansatz eine wichtige Komponente in der fortdauernden Diskussion um die Justiziabilität von WSK-Rechten. Basierend auf der Annahme, dass a) jeder in der Lage sein sollte, den Kerngehalt der WSK-Rechte jederzeit prioritär wahrzunehmen, und b) dass die Nichtumsetzung von Kerngehalten eine prima facie-Verletzung des IPwskR darstellt, ${ }^{19}$ sollten Personen, die ihre Kernrechte nicht wahrnehmen können, mindestens das Recht haben, diese Kernrechte vor nationalen oder internationalen Gerichten oder gerichtsähnlichen Einrichtungen geltend zu machen. ${ }^{20}$ Folglich verspricht der Kernbereichsansatz auch dazu beizutragen, dass programmatische sozioökonomische Forderungen in konkrete, justiziable individuelle WSK-Rechtsansprüche umgewandelt werden. ${ }^{21}$

Wie im folgenden Teil zu zeigen ist, wird der Nutzen des Kernbereichsansatzes allerdings von Einigen in Frage gestellt; ${ }^{22}$ zudem hat der UN-Sozialausschuss seine Konzeption des Ansatzes bislang nicht besonders klar formuliert. Diese Unklarheiten in der Konzeption des Ausschusses und die Kritik am Kernbereichsansatz werden im folgenden Teil aufgegriffen.

Selbst wenn der Kernbereich des Rechts auf Gesundheit nicht im Detail auf internationaler Ebene definiert werden kann, sodass er variierende Gegebenheiten abdeckt, scheint es dennoch sinnvoll, in der Analyse der Verpflichtungen zur »schrittweisen Umsetzung « des Rechts auf Gesundheit die Existenz eines Kernbereichs anzunehmen. Wenn dies nicht der Fall wäre, hätten Einzelpersonen, die in sehr schwierigen wirtschaftlichen und sozialen Umständen leben, weder unmittelbar noch in sehr naher Zukunft ein Recht auf Zugang zu medizinischer Grundversorgung, ohne die ein menschenwürdiges Leben schlecht denkbar ist. ${ }^{23}$ Der Kernbereichsansatz

19 E/1991/23, 14. Dezember 1990, para. 10.

20 Vgl. z.B. E/CN.12/1999/5, 12. Mai 1999, para. 34; E/C.12/2000/4, 11. August 2000, para. 60; E/C.12/GC/18, 6. Februar 2006, para. 49 und E/C.12/2002/11, 20. Januar 2003, para. 57.

21 So argumentieren viele, die den Kernbereichsansatz befürworten, z.B. Bilchitz (2002), 491, De Vos (2002), 24-26, Chapman (2002), 37, Liebenberg (2002), 175, Scott/Macklem (1992), 77 und Alston (1987), 353.

22 Vgl. z.B. Lehmann (2006), Kende (2004) und Porter (2005), 48-55.

23 Das heißt nicht notwendigerweise, dass jeder ein Recht auf kostenfreie Dienstleistungen und Güter hat, die vom Staat bereitgestellt werden müssen. 
impliziert, dass Einzelpersonen ein Recht auf mehr als eine »angemessene Politik« haben, die lediglich darauf abzielt, drängende menschliche Bedürfnisse, auf die sich der Kernbereich stützt, in einem unbestimmten Zeitrahmen zu befriedigen. ${ }^{24}$ Ein Recht auf Gesundheit, dessen Inhalt sich auf ein Recht auf eine bestimmte (Gesundheits-)Politik begrenzt, könnte Staaten leicht einen Anlass geben, die Umsetzung dieses Rechts und anderer WSK-Rechte auf unbestimmte Zeit hinauszuschieben. Die Annahme, dass jedes WSK-Recht einen Kerngehalt besitzt, der unmittelbar und prioritär implementiert werden muss, harmoniert auch mit der teleologischen Interpretationsmethode, die vornehmlich bei der Interpretation internationaler Menschenrechtsstandards angewandt wird. Sie zielt darauf ab, Menschenrechte »nicht nur theoretisch, sondern auch in der Realität « ${ }^{25} \mathrm{zu}$ schützen. Das spiegelt sich zudem in der Bemerkung des UN-Sozialausschusses, dass der IPwskR ohne die Annahme des Kernbereichsansatzes seine raison d'être verlieren würde.

24 Dies bedeutet, dass der Kernbereichsansatz dazu beitragen würde, von einem sog. »meta-rights approach« wegzukommen, wie er von Amartya Sen entwickelt wurde. Sen argumentiert, dass wenn ein bestimmtes Recht $\mathrm{x}$ wegen mangelnder Ressourcen nicht unmittelbar umgesetzt werden kann, haben Einzelpersonen dennoch ein Recht »to have policies $\mathrm{p}(\mathrm{x})$ that genuinely pursue the objective of making the right $x$ realisable«, vgl. Sen (1984), 70. Siehe auch Orago (2015), für die Diskussion wie der Kernbereichsansatz zur effektiven Umsetzung der WSKRechte in der kenianischen Verfassung von 2010 und damit zur Armutsbekämpfung beitragen könnte. Kenia hat ein hohes Niveau von Ungleichheit und Armut zu verzeichnen, selbst im Vergleich mit anderen afrikanischen Staaten.

25 ECSR, Beschwerde 1/1998, International Commission of Jurists v Portugal, Entscheidung vom 9. September 1999, para. 32. 


\section{OfFENE KONZEPTIONELLE Fragen ZUM KERNBEREICHSANSATZ}

Die wichtigsten offenen Fragen zum Kernbereichsansatz sind die Fragen, ob es a) überhaupt möglich ist, den Kerngehalt des Rechts auf Gesundheit in notwendiger Präzision zu bestimmen, und b) auf welcher Ebene das geschehen soll - national oder international. ${ }^{26}$ Diese Fragen hängen auch mit der Frage zusammen, ob Kernverpflichtungen schrittweise realisiert werden sollen, unter maximaler Verwendung bereitstehender Mittel, oder ob man annehmen kann, dass ihre Umsetzung per se für alle Staaten erschwinglich ist und deshalb die Umsetzung von Kernverpflichtungen nicht dem Vorbehalt der »schrittweisen (ressourcenabhängigen) Umsetzung " unterliegt. Es ist des Weiteren unklar, ob der Kernbereich des Rechts auf Gesundheit in Übereinstimmung mit Artikel 4 IPwskR oder durch sog. »retrogressive Maßnahmen « (retrogressive measures) eingeschränkt werden kann. ${ }^{27}$ Der Standpunkt des UN-Sozialausschusses zu diesen Fragen ist unklar. Beispielsweise hat der Ausschuss festgestellt, dass:

»any assessment as to whether a state has discharged its minimum core obligations must take into account the resource constraints applying within a country concerned. $^{28}$

Diese Aussage ist nur verständlich, wenn angenommen wird, dass Vertragsstaaten erst dann ihre Kernverpflichtungen verletzen, wenn sie es versäumen, konkrete Maßnahmen zur Umsetzung der Kernrechte unter effizienter Nutzung aller verfügbaren Mittel zu ergreifen; und dass die Umsetzung von Kernverpflichtungen deshalb dem Vorbehalt der ressourcenabhängigen »schrittweisen Umsetzung u unterliegt. Zudem fordert der Sozialausschuss in seinen Leitlinien zur Berichterstattung, dass Staaten nationale Ziele (Benchmarks) festlegen, um die schrittweise Umsetzung von WSK-

26 Siehe z.B. Lehmann (2006), 183 und Ssenyonjo (2009), 66.

27 Für eine detaillierte Diskussion der Möglichkeiten, WSK-Rechte einzuschränken, siehe Müller (2009).

28 E/1991/23, 14. Dezember 1990, para. 10. 
Rechten zu messen. ${ }^{29}$ In seinen Abschließenden Bemerkungen zu Staatenberichten findet der Ausschuss zudem sehr selten, dass Staaten ihre Kernverpflichtungen verletzt haben, selbst bei Staaten, in denen Menschen hungern und keinen Zugang zu elementarster Gesundheitsversorgung haben; der Ausschuss fordert die Staaten nur sehr vereinzelt direkt auf nachzuweisen, welche unverzüglichen und vorrangigen Abhilfemaßnahmen sie getroffen haben, um Verletzungen von Kernverpflichtungen zu beheben. ${ }^{30}$ Dies deutet darauf hin, dass der Ausschuss der Meinung ist, der Kerngehalt und zugehörige Kernverpflichtungen aus dem Recht auf Gesundheit seien länderspezifisch und ihr Umfang sei von vorhandenen Ressourcen abhängig. Andererseits deuten andere Stellungnahmen des Ausschusses darauf hin, dass der Ausschuss den Kerngehalt eines jeden WSK-Rechts als eine absolute und universelle Untergrenze sieht, unter welcher sich keine Person befinden sollte, und dass Kernverpflichtungen deshalb zu jeder Zeit nachgekommen werden muss, unabhängig vom Stand der wirtschaftlichen Entwicklung des jeweiligen Vertragsstaates und der vorhandenen Ressourcen. In seiner Allgemeinen Bemerkung Nr. 14 zum Recht auf Gesundheit hat der Ausschuss beispielsweise erklärt, dass »a state party cannot, under any circumstances whatsoever [Hervorhebung A.M.], justify its non-compliance with the core obligations [...]. « ${ }^{31}$ Dies hat er in Allgemeinen Bemerkungen zu anderen Artikeln des Sozialpakts wiederholt. ${ }^{32}$

29 Die Leitlinien zur Berichterstattung des UN-Sozialausschusses fordern die Staaten auf anzugeben, ob sie eine nationale Armutsgrenze formuliert haben und auf welcher Basis diese berechnet wird. Falls Staaten keine nationale Armutsgrenze definiert haben, fragt der Ausschuss regelmäßig, welche Mechanismen genutzt werden, um die Verbreitung und das Ausmaß an Armut $\mathrm{zu}$ messen. E/C.12/2008/2, 24. März 2009, paras. 3 (b) und 42 sowie E/CN.12/1999/5, 12. Mai 1999, para. 21.

30 Vgl. z.B. E/C.12/1/Add.48, 1. September 2000, para. 25; genauso: E/C.12/1/Add. 95, 12. Dezember 2003; Ausnahmen sind: E/C.12/COD/CO/4, 20. November 2009, para. 16, in welcher der Ausschuss von »schwerwiegenden Verletzungen« des Artikel 2 (1) IPwskR spricht, und E/C.12/LKA/CO/2-4, 9. Dezember 2010, para. 28.

31 E/C.12/2001/10, 10. Mai 2001, paras. 47-48.

32 E/CN.12/1999/5, 12. Mai 1999, para. 6; E/C.12/2002/11, 20. Januar 2003, paras. 40, 42 und 44 (c) sowie E/C.12/GC/19, 4. Februar 2008, para. 65. 
Auch in der Literatur werden Argumente entweder für die Festlegung von länderspezifischen Kerngehalten, die einer "schrittweisen Umsetzung « unterliegen, vorgebracht oder für die Festlegung von universellen Kerngehalten, von denen angenommen wird, dass ihre Umsetzung per se für alle Vertragsstaaten erschwinglich ist, einschließlich einkommensschwacher Länder.

Die Hauptargumente jener, die einen national definierten Kerngehalt stützen, sind erstens, dass es angesichts der großen Entwicklungsunterschiede weltweit unrealistisch sei zu fordern, dass alle Staaten dieselben Kernverpflichtungen umsetzten. ${ }^{33}$ Einkommensschwache Länder verfügten nicht über ausreichende Mittel, um beispielsweise das Kernrecht auf Gesundheit, wie es vom UN-Sozialausschuss definiert ist, allen ihrer Jurisdiktion unterstehenden Personen zu garantieren; ${ }^{34}$ für einkommensstarke Länder hingegen könnte ein universell definierter Kernbereich des Rechts auf Gesundheit politische Trägheit fördern, obwohl diese Länder eigentlich weitreichendere (Nicht-Kern-)Verpflichtungen ohne größere Probleme umsetzen könnten. ${ }^{35}$

Zweitens wird argumentiert, dass ein länderspezifischer Kerngehalt des Rechts auf Gesundheit definiert werden sollte, weil ein universell definierter Kerngehalt unweigerlich zu abstrakt und inflexibel sei. Ein universell definierter Kernbereich trenne die Theorie zum Recht auf Gesundheit von den tatsächlichen Erfahrungen, die Einzelpersonen machten, deren Recht auf Gesundheit verletzt ist. Dies könne zu einem ungewollten Ausschluss einiger Personen vom Schutzbereich des Kernrechts auf Gesundheit führen,

33 Siehe Craven (1995), 141 und Scott/Alston (2000), 250.

34 Aus diesem Grund hat das südafrikanische Verfassungsgericht den Kernbereichsansatz abgelehnt. Es hat festgestellt, dass es für den südafrikanischen Staat nicht möglich ist, Kernleistungen unverzüglich zur Verfügung zu stellen, z.B. South Africa: Constitutional Court (2002), paras. 34-37; siehe auch Kende (2004), 622 und Chapman/Russell (2002) 10, die auf diese Gefahr hinweisen.

35 Siehe z.B. van Bueren (1999), 59, Chapman/Russel (2002), 9, Ssenyonjo (2009) 66-67 und Craven (1995), 144, der die Sorge nennt, dass - wenn der UN- Sozialausschuss sich zu sehr auf den Kernbereichsansatz konzentriert - , er seine primäre Aufmerksamkeit notwendigerweise auf die Situation in einkommensschwachen Ländern lenkt. Dies könnte die Arbeit des Ausschusses weiterer Kritik aussetzen. 
wenn die kontextspezifischen Erfahrungen dieser Personen sich nicht im universell definierten Kerngehalt widerspiegelten. ${ }^{36}$

Drittens erfährt die Festlegung von länderspezifischen Kerngehalten Unterstützung durch die Tatsache, dass Zweifel bestehen, ob es überhaupt möglich sei, einen (tatsächlich) universellen Kernbereich des Rechts auf Gesundheit und dazugehörige universelle Kernverpflichtungen zu bestimmen. Diese Zweifel ergeben sich, weil bisher keine überzeugenden Kriterien gefunden wurden, die helfen, Kern- und Nicht-Kern-Elemente des Rechts auf Gesundheit und anderer WSK-Rechte plausibel voneinander abzugrenzen. In ihrer umfassenden Analyse von Werten und Bedürfnissen, die regelmäßig als Grundlage für die Entwicklung von Kerngehalten dienen, kommt Young beispielsweise zu dem Schluss, dass es »keine Axiome gibt, auf die sich ein unumstrittener Kerngehalt gründen könnte«. ${ }^{37}$

Bezugnehmend auf verschiedene Versuche, Kerngehalte über normative Argumente zu definieren, schlussfolgert Young, dass

»the minimum core will look different to an advocate of human flourishing in comparison with an advocate of basic survival, just as the core will look different in various instantiations of both survival and dignity. ${ }^{38}$

Diese Schwierigkeit zeigt sich auch in der Herausforderung, numerische Formeln zu erstellen, die eine Implementierung von universellen Kernverpflichtungen in allen Ländern messbar machen. ${ }^{39}$ Ohne solche Formeln ist es schwer für den UN-Sozialausschuss, die staatlichen Bemühungen zur

36 Vgl. Porter (2005), 52; siehe auch Lehmann (2006), 188-189; diese Schwierigkeit wird auch von Liebenberg (2006), 31 und Pieterse (2006), 491 anerkannt.

37 Young (2008) [eigene Übersetzung]. Young analysiert verschiedene, auf Grundbedürfnissen (Leben, Überleben) und Werten (Würde, Gleichheit, Freiheit) basierende Ansätze, die darauf abzielen, einen universell akzeptierten Kerngehalt der WSK-Rechte zu entwickeln.

38 Young (2008), 138; sogar Bilchitz (2007), 224, ein starker Verfechter des universellen Kerngehalts der WSK-Rechte, den er auf grundlegenden menschlichen Bedürfnissen aufbaut (dem Überlebensinteresse), erkennt an, dass das menschliche Überlebensinteresse nicht als eine geeignete Grundlage für die Bestimmung des Kerngehalts des Rechts auf Gesundheit dienen kann. 
Umsetzung universeller Mindestverpflichtungen aus dem Kernrecht auf Gesundheit zu beurteilen.

Es folgen nun die Argumente jener, die vorbringen, dass die Festlegung eines Kerngehalts des Rechts auf Gesundheit nur sinnvoll sei, wenn dieser Kerngehalt als ein universeller, unabänderbarer Standard verstanden werde, den alle Vertragsstaaten des IPwskR jederzeit umsetzen müssten, unabhängig vom Entwicklungsstand des jeweiligen Landes. ${ }^{40}$

Erstens wird hervorgehoben, dass nur ein universell definierter Kerngehalt den obengenannten originären Zweck des Kernbereichsansatzes erfüllen könne, der Verpflichtung zur »schrittweisen Umsetzung« eine größere Bestimmtheit zu verleihen. Es bestünde kein Unterschied zwischen einer Verpflichtung, nationale Kernbereiche zu bestimmen, und der generellen Verpflichtung unter Artikel 2 (1) IPwskR, WSK-Rechte schrittweise umzusetzen, da der Umfang der Verpflichtungen jeweils von verfügbaren Ressourcen abhängig gemacht würde.

Zweitens bezögen sich die meisten Aspekte der Kernbereiche von WSK-Rechten, wie sie vom UN-Sozialausschuss in seinen Allgemeinen Bemerkungen definiert sind, auf den Schutz von überlebenswichtigen Interessen, die alle Menschen teilen, unabhängig davon, welche Ressourcen vorhanden seien ${ }^{41}$ und ob deren Umsetzung eine Grundvoraussetzung für eine menschenwürdige Existenz und Entwicklung sei. ${ }^{42}$ Die Umsetzung dieser Kernverpflichtungen solle unweigerlich zu den staatlichen Grundaufgaben in allen Ländern gehören, ${ }^{43}$ und Staaten - sogar einkommens-

40 Z.B. Bueren (2002), 184, Russell (2002), 15, OHCHR (1986), para. 25, Chapma/Russell (2002), 14, Bilchitz (2007), Kap. 6, Arambulo (1999), 130-135, Scott/Alston (2000), 250, Scott/Macklem (1992), 77 und Ssenyonjo (2009), 66.

41 Vgl. Bilchitz (2007), 222.

42 Dies hat das Schweizerische Bundesgericht überzeugend dargelegt: Schweizerisches Bundesgericht (1995), Erwägung 2, para. b: »Die Sicherung elementarer menschlicher Bedürfnisse wie Nahrung, Kleidung und Obdach ist die Bedingung menschlicher Existenz und Entfaltung überhaupt.« Siehe auch Bilchitz (2007), 187.

43 Wie von Chapman/Russell (2002), 11-12 angemerkt; siehe auch die Entscheidung des Supreme Court of India (1996), para. 16, in der vom Supreme Court bemerkt wird, dass, wenn etwas verfassungsmäßig vorgeschrieben ist (in diesem 
schwache Staaten - »müssen irgendwo [mit der Umsetzung von WSKRechten] beginnen «. ${ }^{44}$ Die Bereitstellung von grundlegender Gesundheitsversorgung für sozioökonomisch benachteiligte Gruppen durch die Umsetzung der Kernverpflichtungen aus dem Recht auf Gesundheit könne ein guter Anfangspunkt sein. ${ }^{45}$ Es sei deshalb vertretbar anzunehmen, dass die Umsetzung von universellen Kernverpflichtungen auch für einkommensschwache Länder erschwinglich sei, wenn nötig mittels internationaler Kooperation und Hilfe.

In Zusammenhang mit diesem Punkt wird drittens hervorgehoben, dass nur ein universell definierter Kerngehalt des Rechts auf Gesundheit verspreche, effektiv dazu beizutragen, nationale Verpflichtungen von internationalen Verantwortlichkeiten für die Umsetzung des Rechts auf Gesundheit abzugrenzen. Unter Bezugnahme auf internationale Maßnahmen zur Armutsbekämpfung hat der UN-Sozialausschuss festgestellt, dass

»[...] grouped together, the core obligations establish an international minimum threshold that all developmental policies should be designed to respect. In accordance with General Comment No. 14, it is particularly incumbent on all those who can assist, to help developing countries respect this international minimum threshold. If a national or international anti-poverty strategy does not reflect this

Fall die Bereitstellung von medizinischen Notfalldiensten für alle indische Staatsbürgerinnen und -bürger), dies umgesetzt werden muss, auch wenn die verfügbaren Ressourcen begrenzt sind.

44 Wie von Dankwa, Professor für Rechtswissenschaften an der Universität von Ghana und (ehem.) Mitglied der Afrikanischen Kommission für Rechte der Menschen und Völker vertreten, siehe COHRE (2003), 119 [eigene Übersetzung]; genauso Wesson (2004), 299.

45 Dass diese Ansicht von einigen Staaten geteilt wird, offenbart sich in den Berichten der Open-ended Working Group to Consider Options Regarding the Elaboration of an Optional Protocol to the ICESCR. Einige Delegationen, die an den Konsultationen zur Ausarbeitung des Zusatzprotokolls zum IPwskR teilgenommen haben, merkten an, dass die Zuteilung ausreichender Mittel für die Umsetzung von Kernverpflichtungen eine unmittelbare Verpflichtung aller Vertragsstaaten sei. Siehe Report of the Open-ended Working Group, first session, E/CN.4/2004/44, 15. März 2004, para. 56. Der Bericht legt nicht offen, wie viele Delegationen diese Meinung teilten. 
minimum threshold, it is inconsistent with the legally binding obligations of the State party. ${ }^{46}$

Nigel Whites Vorschlag, dass der UN-Sicherheitsrat sicherstellen sollte, dass Kernverpflichtungen aus allen WSK-Rechten durch Maßnahmen, die er anordnet, nicht beeinträchtigt werden - beispielsweise bei der Verhängung von Sanktionen -, deutet auf ein universelles Verständnis von Kerngehalten und -verpflichtungen hin. White schreibt:

»the effect of Security Council resolutions is not to free the target state, other member states, or itself from compliance with basic [Hervorhebung A.M.] economic and social rights «. ${ }^{47}$

Diese Zusammenfassung der Hauptargumente zeigt, dass beide Optionen die Definition von länderspezifischen oder universellen bzw. internationalen Kerngehalten - Probleme in sich bergen.

Die Definition ressourcenabhängiger länderspezifischer Kernverpflichtungen aus dem Recht auf Gesundheit, die nur nach und nach implementiert werden müssen, verhindert, dass der Kernbereichsansatz sein ursprüngliches Ziel erreicht - einen gewissen Grad an Bestimmtheit der staatlichen Verpflichtungen unter Artikel 2 (1) und 12 des IPwskR durch die Definition eines universellen Mindeststandards, der es dem UN-Sozialausschuss ermöglicht, die Eignung und Angemessenheit staatlicher Umsetzungsmaßnahmen zu evaluieren, unabhängig vom Entwicklungsstand des jeweiligen Landes. Eine Verpflichtung, einen länderspezifischen Kerngehalt des Rechts auf Gesundheit zu definieren im Einklang mit bereitstehenden Ressourcen, und das Festlegen nationaler Orientierungswerte, um dessen Umsetzung zu kontrollieren, scheint sich nicht von der generellen Verpflichtung zur »schrittweisen Umsetzung « des Rechts auf Gesundheit unter Artikel 2 (1) und 12 IPwskR zu unterscheiden.

Angesichts der beträchtlichen wirtschaftlichen, sozialen, kulturellen und politischen Unterschiede zwischen den Staaten wirft der Versuch, einen universell bestimmten Kernbereich des Rechts auf Gesundheit festzulegen, allerdings auch Probleme auf. Es scheint illusorisch, dass ein universeller

46 E/C.12/2001/10, 10. Mai 2001, para. 17.

47 White (2007), 102 und Young (2008), 122-123. 
Kerngehalt genügend detailliert bestimmt werden kann, sodass er die Erfahrungen verschiedenster Personen aus aller Welt abdeckt. Insbesondere scheint es problematisch anzunehmen, dass die Umsetzung international definierter Kernbereiche für alle Länder erschwinglich ist, besonders solange es nicht klar ist, wie Umsetzungsverpflichtungen und -verantwortlichkeiten nationalen und internationalen Akteuren zugeordnet und koordiniert werden sollen. Vor allen Dingen scheint die Umsetzung des universellen Kernrechts auf Gesundheit, wie es vom UN-Ausschuss in den Allgemeinen Bemerkungen Nr. 14 definiert ist, unerschwinglich für viele einkommensschwache Länder. Nach der Definition des Ausschusses umfasst der Kernbereich nicht nur ein Recht auf Zugang zu medizinischer Grundversorgung, sondern auch zu den zugrunde liegenden Determinanten von Gesundheit wie Zugang zu ausreichend Nahrung, Wasser, angemessener Sanitärversorgung und Unterkunft. ${ }^{48}$

\section{SYNTHESE: \\ DIE BEZIEHUNG ZWISCHEN EINEM UNIVERSELL DEFINIERTEN KERNGEHALT DES RECHTS AUF GESUNDHEIT UND EINEM PRAGMATISCHEN, LÄNDERSPEZIFISCHEN KERNGEHALT}

Vor diesem Hintergrund soll im Folgenden eine Alternative entwickelt werden, eine Synthese aus dem universell definierten und dem länderspezifischen Kerngehaltsansatz: Es soll ein sinnvolles Verhältnis zwischen einem generelleren, universell definierten Kerngehalt und einem konkreteren, national bestimmten Kerngehalt des Rechts auf Gesundheit skizziert werden, u.a. gemäß eines von David Bilchitz angedeuteten Vorschlags. ${ }^{49}$ Die Synthese basiert auch auf einer ersten Analyse der Rechtsprechung zum Recht auf Gesundheit nationaler Gerichte, des Europäischen Ausschusses für Soziale Rechte (ECSR), des Europäischen Gerichtshofs für Menschenrechte (EGMR) und der Bemerkungen des UN-Fachausschusses für den Sozialpakt.

48 E/C.12/2000/4, 11. August 2000, paras. 43-44.

49 Vgl. Bilchitz (2007), 220-225. Ähnlich Koch (2009), 280-288, Pieterse (2006), 491 und Liebenberg (2005). 
Ein international definierter Kerngehalt würde die »Mindestanforderungen an das Recht auf Gesundheit « (»minimum essential levels of [...] the right $\left[\ldots\right.$ to health $«^{50}{ }^{\text {) }}$ beschreiben, wie sie in den Allgemeinen Bemerkungen Nr. 14 des UN-Sozialausschusses definiert sind. Ein Minimum an Ressourcen wäre für ihre Umsetzung nötig, um sicherzustellen, dass zumindest die lebensbedrohenden Gesundheitsgefahren beseitigt sind. Es wird stark angenommen, dass jeder Staat Zugang zu diesen Ressourcen hat, wenn nötig durch internationale Kooperation und Hilfeleistungen. Die weitestgehende Verknüpfung der »Mindestanforderungen an das Recht auf Gesundheit« mit internationalen Indikatoren würde zur effektiven Überwachung staatlicher Umsetzungsmaßnahmen beitragen. Dies würde es auch erlauben, dem Hauptziel, das der UN-Sozialausschuss mit der Annahme des Kerngehaltsansatzes erreichen wollte, näher zu kommen, nämlich der Tendenz entgegenzuwirken, dass Staaten tatsächlichen, rechtlich-bindenden Verpflichtungen unter dem Sozialpakt entgehen, mit dem Hinweis auf die »bloß weiche« Verpflichtung, die WSK-Rechte im Sozialpakt »nach und nach« umzusetzen.

Gleichzeitig wäre jeder Staat verpflichtet, einen pragmatischen und konkreten Kerngehalt des Rechts auf Gesundheit auf nationaler Ebene zu definieren, in Übereinstimmung mit vorhandenen Ressourcen. Diese Definition sollte vom generell formulierten universellen Kerngehalt geleitet werden, der an die vorherrschenden nationalen Gegebenheiten angepasst wird und sich damit im national definierten Kerngehalt kontextspezifisch konkretisiert. Die im jeweiligen Land vorhandenen finanziellen Ressourcen und fachlichen Kapazitäten würden bestimmen, ob der länderspezifische Kerngehalt mit dem universell definierten Kerngehalt übereinstimmt. Wenn dies nicht der Fall ist, würde der universell definierte Kernbereich dazu dienen hervorzuheben, dass die Annäherung des national definierten Kernbereiches an den universell definierten Kernbereich oberste Priorität haben muss. Dies leitet sich aus der Tatsache ab, dass der universell definierte Kerngehalt auf fundamentalen menschlichen Bedürfnissen und Interessen aufbaut. Je mehr Ressourcen und Kapazitäten die Umsetzung verschiedener Aspekte des international definierten Kerngehalts erfordert, desto schwerer ist es für einkommensschwache Staaten, ihren länderspezifischen Kernbereich an den universellen anzupassen. Einkommensstarke Staaten, deren

50 E/1991/23, 14. Dezember 1990, para. 10. 
länderspezifisch definierter Kerngehalt bereits mit dem universell definierten Kernbereich übereinstimmt, wären verpflichtet, mit der Umsetzung von Bereichen des Rechts auf Gesundheit, die über den Kernbereich hinausgehen (Nicht-Kernbereiche), fortzufahren.

Die Bestimmung einer sinnvollen Beziehung zwischen einem universell definierten Kernbereich und einem pragmatischen, länderspezifischen Kerngehalt des Rechts auf Gesundheit würde einerseits sicherstellen, dass das Recht auf Gesundheit und andere WSK-Rechte einen aussagekräftigen Inhalt bekommen, trotz der Verpflichtung im Sozialpakt, diese Rechte »bloß« »nach und nach« bzw. »schrittweise« umzusetzen. Andererseits würde eine solche sinnvolle Beziehung anerkennen, dass es unmöglich ist, einen universellen Kerngehalt des Rechts auf Gesundheit so präzise zu bestimmen, dass er die sehr unterschiedlichen Gesundheitsprobleme in verschiedenen Ländern abdeckt und dass seine Präzisierung deshalb auf nationaler Ebene stattfinden sollte, die unterschiedlichen Faktoren, einschließlich vorhandener Ressourcen und fachlichen Kapazitäten, Rechnung tragen kann. Ein solches Verständnis des Kernbereichsansatzes würde mit dem Wortlaut des Artikel 2 (1) IPwskR übereinstimmen, da eine Einhaltung der Verpflichtung zur »schrittweisen Umsetzung « unter maximaler Verwendung aller verfügbaren Mittel ohne das Definieren eines Minimalstandards, der den Startpunkt dieser »schrittweisen Umsetzung« ausmacht, kaum denkbar ist. ${ }^{51}$

Einige Aussagen des UN-Sozialausschusses (nicht ausschließlich zum Recht auf Gesundheit), scheinen eine solche »Aufspaltung « in einen pragmatischen, länderspezifischen Kerngehalt und einen universellen Kernbereich zu unterstützen. Am deutlichsten wird dies in den Allgemeinen Bemerkungen Nr. 19, in denen der Ausschuss zunächst die staatlichen Kernverpflichtungen aus dem Recht auf soziale Sicherheit definiert:

»to ensure access to a social security scheme that provides a minimum essential level of benefits to all individuals and families that will enable them to acquire at least essential health care, basic shelter and housing, water and sanitation, foodstuffs, and the most basic forms of education. ${ }^{52}$

51 Vgl. Koch (2009), 285.

52 E/C.12/GC/19, 4. Februar 2008, para. 59 (a). 
Entscheidend ist, dass der UN-Sozialausschuss dann fortfährt mit der Bemerkung, dass

»if a state party cannot provide this minimum level for all risks and contingencies within its maximum available resources, the Committee recommends that the State party, after a wide process of consultation, select a core group of social risks and contingencies. ${ }^{53}$

Auch die Allgemeine Bemerkung Nr. 14 zum Recht auf Gesundheit definiert erstens den Kerngehalt des Rechts auf Gesundheit und die zugehörigen Kernverpflichtungen, und zweitens »Verpflichtungen vergleichbarer Priorität« (obligations of comparable priority) unter der Überschrift »Kernverpflichtungen «. ${ }^{54}$ Dies scheint darauf hinzudeuten, dass der Ausschuss davon ausgeht, dass nicht alle universell definierten Kernverpflichtungen aus dem Kernrecht auf Gesundheit gleich von allen Staaten umgesetzt werden können. ${ }^{55}$

Abbildung 1 fasst die hier skizzierte Beziehung zwischen einem universell definierten Kernbereich und einem konkreten, länderspezifischen Kernbereich zusammen. Der Kasten unten listet des Weiteren verfahrensbezogene Verpflichtungen auf, die für die Bestimmung detaillierter materieller Kernverpflichtungen auf nationaler Ebene wichtig sind. Im Folgenden soll die Funktion und Interaktion zwischen dem universell bestimmten und dem national definierten Kernbereich sowie die Rolle verfahrensbezogener Verpflichtungen näher beleuchtet werden. Das Hauptziel dabei ist zu verstehen, wie dieses Zusammenspiel und die Umsetzung verfahrensbezogenen Verpflichtungen dazu beitragen können, mindestens den Kerngehalt des Rechts auf Gesundheit in unterschiedlichen Ländern weltweit zu realisieren.

53 Ebd., para. 59 (a).

54 E/C.12/2000/4, 11. August 2000, paras. 43-44.

55 Ähnliches kann geschlossen werden aus der E/1991/23, 14. Dezember 1990, para. 11, in der es heißt, dass »even where the available resources are demonstrably inadequate [to implement core obligations at once], the obligation remains for a State party to strive to ensure the widest possible enjoyment of the relevant rights under the prevailing circumstances [Hervorhebung A.M.]«. 


\section{Abbildung 1: Die Beziehung zwischen einem universell definierten und einem länderspezifischen Kerngehalt des Rechts auf Gesundheit}

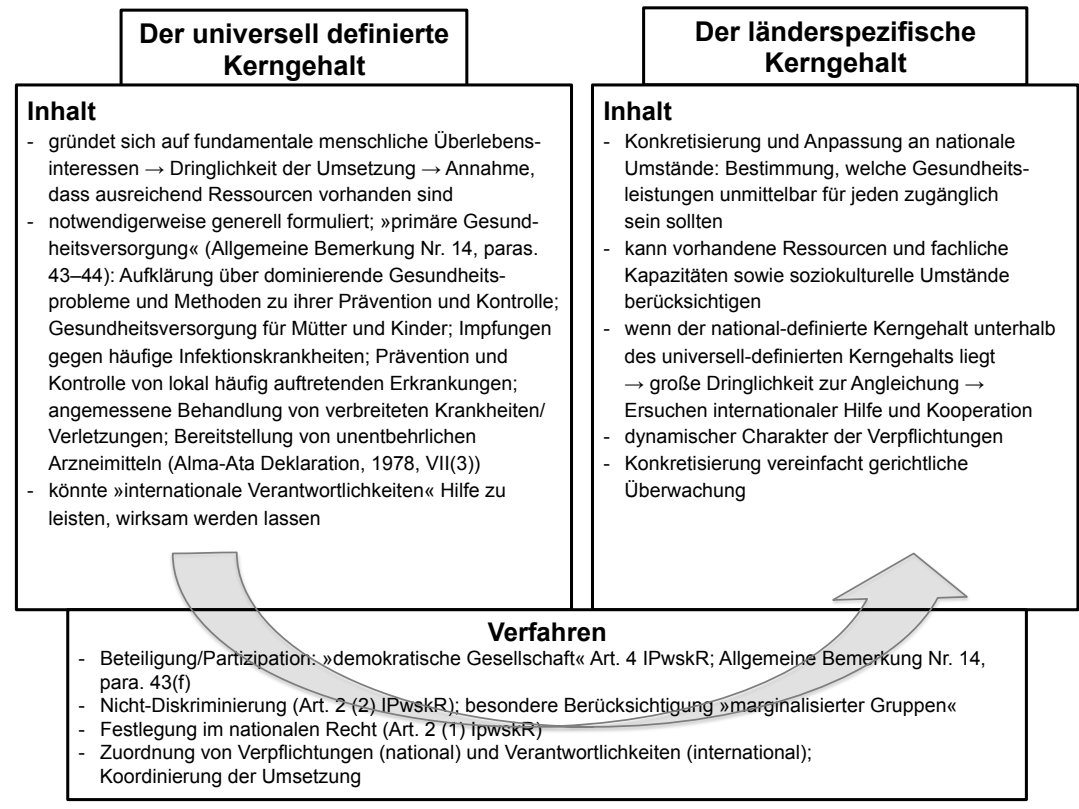

Quelle: Eigene Darstellung 


\subsection{Der universell definierte Kernbereich des Rechts auf Gesundheit}

Der universell definierte Kerngehalt des Rechts auf Gesundheit würde die zu erfüllenden »Mindestanforderungen « (minimum essential levels ${ }^{56}$ ) dieses Rechts beschreiben, wie sie der UN-Sozialausschuss in seinen Allgemeinen Bemerkungen Nr. 14 bestimmt hat. Wie Bilchitz überzeugend und ausführlich argumentiert, sind es zwingende menschliche Interessen und Bedürfnisse, allen voran das Überlebensinteresse, auf welchen der internationale Kernbereich des Rechts auf Gesundheit und anderer WSK-Rechte aufbaut. $^{57}$ Das Überlebensinteresse kann beispielsweise als Grundlage für die Festlegung der internationalen Kerngehalte der Rechte auf Nahrung, Wasser, soziale Sicherheit und angemessene Unterbringung dienen, da es möglich ist, intersubjektiv in etwa zu bestimmen, zu welchen Gütern ein Mensch Zugang haben sollte (Menge an Kalorien pro Tag, Liter Wasser pro Tag, Höhe von sozialen Geldleistungen usw.), um sein/ihr Überleben zu sichern. Die speziellen Eigenschaften des Rechts auf Gesundheit machen es allerdings problematisch, das menschliche Überlebensinteresse als Basis für den universellen Kerngehalt des Rechts auf Gesundheit aller Menschen zu nehmen, zumindest wenn seine Umsetzung auch in einkommensschwachen Staaten realistisch sein soll. ${ }^{58}$ Wegen des stark variierenden Gesundheitszustandes jedes Einzelnen müsste ein individueller Kerngehalt des Rechts auf Gesundheit (unter dem ein unmittelbarer Zugang zu bestimmten Gesundheitsleistungen gegeben werden muss) für jeden Menschen bestimmt werden, wenn man direkt das menschliche Überlebensinteresse als Grundlage für diesen Kerngehalt nehmen würde. Eine prioritäre Umsetzung einiger dieser individuellen Kernrechte von Personen mit sehr seltenen Krankheiten könnte den Gesundheitshaushalt besonders von einkommensschwachen Ländern stark beeinflussen. Es scheint deshalb notwendig, dass der Inhalt des universellen Kernrechts auf Gesundheit mit Hilfe von utilitaristi-

56 E/1991/23, 14. Dezember 1990, para. 10.

57 Vgl. Bilchitz (2007), 222. Der ECSR nennt die Menschenwürde als fundamentalen Wert, der dem Recht auf Gesundheit zugrunde liegt: International Federation of Human Rights Leagues (FIDH) v. France, Complaint No. 14/2003, 3. November 2004, para. 31.

58 Bilchitz (2007), 220-225 erkennt dies auch an. 
schen Erwägungen bestimmt wird, sodass der Kernbereich Rechte auf Zugang zu Gesundheitsleistungen und -gütern garantiert, die es der großen Mehrheit der Menschen ermöglicht, ihre Überlebensinteressen durch diesen Zugang zu wahren. ${ }^{59}$ Dieser Ansatz scheint sich in der Definition des universellen Kerngehalts des Rechts auf Gesundheit in den Allgemeinen Bemerkungen Nr. 14 des UN-Sozialausschusses und der Deklaration von Alma-Ata zu Primärer Gesundheitsversorgung von $1978^{60}$ widerzuspiegeln, wenn diese den Kerngehalt als ein Recht auf Zugang zu primärer Gesundheitsfürsorge darstellen.

Obwohl sie es nicht ganz so explizit formulieren wie der UN-Sozialausschuss, scheinen der EGMR und der ECSR ebenfalls davon auszugehen, dass Staaten dazu verpflichtet sind, mindestens den Kernbereich sozialer und wirtschaftlicher Rechte unter den jeweiligen Verträgen, deren Umsetzung diese beiden Institutionen überwachen, zu schützen. In ihrer Analyse der EGMR-Rechtsprechung zum Schutz wirtschaftlicher und sozialer Forderungen merkt Ida Koch beispielsweise an, dass einige Aussagen des EGMR dahingehend interpretiert werden können, dass sie die Anerkennung von Kernrechten zu medizinischer Grundversorgung ${ }^{61}$ und zu grundlegenden Sozialleistungen unter der Europäischen Menschenrechtskonvention ${ }^{62}$ zulassen. ${ }^{63}$ Der ECSR hatte noch nicht die Gelegenheit, sich besonders häu-

59 Dies wird besonders von Lehmann (2006), 190 vertreten.

60 World Health Organization (1978).

61 Koch (2009), 63-64; die u.a. EGMR (2001), para. 219 analysiert; siehe auch Harris et al. (2009), 46-57.

62 Europäische Menschenrechtskonvention, 213 UNTS 222, 4. November 1950.

63 Koch (2009), Kap. 8. Koch nennt Urteile, in denen der EGMR soziale Geldleistungen unter Artikel 3, 6 und 8 EMRK sowie unter dem Recht auf Eigentum im 1. Zusatzprotokoll zur EMRK (Artikel 1) geschützt hat oder in denen er wenigstens nicht ausgeschlossen hat, dass solche Geldleistungen von der Konvention geschützt sind, wenn die Lebensumstände des Beschwerdeführers »a minimum level of severity« erreicht haben. Siehe auch Clements/Simmons (2008), 426, die festhalten, dass "[I]n relation to complaints that disclose gross failures of the most basic socio-economic support, the Court's [ECtHR] starting point is now an unequivocal acceptance of the view that the Convention protects a core irreducible set of such rights«. Dies wurde auch in neueren Urteilen bestätigt, z.B. MSS v. Belgium and Greece, Appl. No. 30696/09, Urteil (Große Kammer), 21. 
fig zur Interpretation des Rechts auf Gesundheit in der (revidierten) Europäischen Sozialcharta (ESC/RESC) ${ }^{64}$ zu äußern. Die »Rechtsprechung« des Ausschusses zu einigen Artikeln deutet aber an, dass ECSR den Mindestschutz eines Kerngehaltes der Rechte in der ESC/RESC befürwortet. So sind Staaten unter Artikel 12 (2) ESC beispielsweise verpflichtet, ein System der sozialen Sicherheit auf einem befriedigenden Stand zu halten, der zumindest dem entspricht, der für die Ratifikation des Übereinkommens (Nr. 102) der Internationalen Arbeitsorganisation (ILO) über die Mindestnormen der sozialen Sicherheit erforderlich ist.

Nach Artikel 12 (2) RESC ist der Minimalstandard auf den Standard des Europäischen Kodex für soziale Sicherheit heraufgesetzt worden. ${ }^{65}$ Artikel 12 (3) ESC/RESC enthalten weitere Verpflichtungen, »sich zu bemühen, das System der sozialen Sicherheit fortschreitend auf einen höheren Stand zu bringen.« Daher lässt sich feststellen, dass der Inhalt eines europäischen Kernrechts auf soziale Sicherheit in beträchtlichem Detail in ILO-Übereinkommen Nr. 102 und dem Europäischen Kodex für Soziale Sicherheit definiert ist. ${ }^{66}$ Da beide Instrumente zudem Regelungen zum

Januar 2011, para. 263 und McCann v. United Kingdom, Appl. No. 19009/04, Urteil (Vierte Sektion) 13. Mai 2008. Der EGMR ist allerdings vorsichtig, seine Rechtsprechung zu sozioökonomischen Fragen nicht zu sehr auszuweiten, siehe z.B. Koufaki and Adedy v. Greece, Appl. Nos. 576657/12 und 557657/12, Entscheidung (Erste Sektion), 7. Mai 2013.

64 Europäische Sozialcharta, 529 UNTS 89, 18. Oktober 1961; Revidierte Europäische Sozialcharta, 2151 UNTS 277, 3. Mai 1993.

65 Artikel 12(2) RESC: „Um die wirksame Ausübung des Rechts auf Soziale Sicherheit zu gewährleisten, verpflichten sich die Vertragsparteien: [...] (2) das System der Sozialen Sicherheit auf einem befriedigenden Stand zu halten, der zumindest dem entspricht, der für die Ratifikation der Europäischen Ordnung der Sozialen Sicherheit erforderlich ist.« Der Europäische Kodex für Soziale Sicherheit hat das ILO Übereinkommen Nr. 102 als Grundlage, fordert aber ein höheres Sozialleistungsniveau. Siehe auch Explanatory Report on the Revised European Social Charter (1996), ETS No. 163, para. 58.

66 Beide Instrumente geben den Staaten jedoch Freiheit in der Wahl der Mittel, mit der sie die relevanten Bestimmungen umsetzen. Die Ziele des ILO-Übereinkommens Nr. 102 und des Europäischen Kodex für Soziale Sicherheit können beispielsweise durch »universal schemes; social insurance schemes with earni- 
Umfang von Gesundheitsleistungen enthalten, können sie auch genutzt werden, um den Inhalt eines europäischen Kernrechts auf Gesundheit zu bestimmen, ${ }^{67}$ zusammen mit der »Rechtsprechung « des ECSR zum Artikel 11 ESC/RESC. ${ }^{68}$ Des Weiteren hat auch die Afrikanische Kommission für die Rechte der Menschen und Völker den Kerngehaltsansatz in ihrer Interpretation der Afrikanischen Charta der Rechte der Menschen und Völker aufgegriffen. $^{69}$

Gerade weil der universelle Kerngehalt des Rechts auf Gesundheit und anderer WSK-Rechte auf fundamentalen menschlichen (Überlebens-) Interessen beruht, ist seine Umsetzung besonders drängend, um weitreichende negative Folgen für menschliches Wohlbefinden zu verhindern. Deshalb gründet sich der Kernbereichsansatz auf die Annahme, dass die Vertragsstaaten des UN-Sozialpakts generell über ausreichend Ressourcen verfügen, die für die Umsetzung von Kernverpflichtungen nötig sind, da außerdem angenommen wird, dass diese Umsetzung $\mathrm{zu}$ wichtigen und grundlegenden Staatsfunktionen gehört.

Der Inhalt eines universell definierten Kernrechts auf Gesundheit und dazugehöriger Kernverpflichtungen ist zwangsläufig eher generell formuliert. Wie bereits erwähnt, ist es unrealistisch zu erwarten, dass der UN-Sozialausschuss den universellen Kernbereich des Rechts auf Gesundheit im Detail definiert oder erschöpfende Richtlinien zu seiner Umsetzung erstellt. $^{70}$ Der UN-Sozialausschuss kann weder alle individuellen Erfahrun-

ngs related or flat rate components or both; or social assistance schemes' erreicht werden (Commentary on the ILO Social Security (Minimum Standards) Convention, 1952 (No. 102), www.ilo.org/secsoc/areas-of-work/legal-advice/ WCMS_205340/lang--en/index.htm [06.01.2016]. Dies unterstützt die »Aufspaltung « in einen national definierten und einen universell definierten Kerngehalt des Rechts auf soziale Sicherheit, wie er in diesem Beitrag vorgeschlagen wird.

67 Artikel 7-18 ILO-Übereinkommen Nr. 102 und Artikel 7-18 Europäischer Kodex für Soziale Sicherheit.

68 Artikel 11 ESC/RESC enthält ein »Recht auf Schutz der Gesundheit«.

69 Siehe die Analyse von Nkongolo (2014), 501-502.

70 Diese Schwierigkeit offenbart sich auch in der »Rechtsprechung « des ECSR zu Artikel 13 (1) ESC, der jedem, der über keine ausreichenden Mittel verfügt, ein Recht auf notwendige medizinische Betreuung gibt. Siehe z.B. ECSR, Schluß- 
gen von Menschen, deren gesundheitliche Grundbedürfnisse nicht befriedigt sind, ${ }^{71}$ noch alle speziellen politischen, wirtschaftlichen, sozialen, kulturellen und anderen Merkmale aller Vertragsstaaten in seine Definition des universellen Kernrechts auf Gesundheit mit einfließen lassen. So wird zum Beispiel angemerkt, dass weder die Definition des universellen Kernrechts auf Gesundheit in den im Jahr 2000 formulierten Allgemeinen Bemerkungen Nr. 14 noch andere Teile der Allgemeinen Bemerkungen explizit die HIV/AIDS-Epidemie als ein drängendes Gesundheitsproblem nennen oder eine Strategie zu seiner Bekämpfung skizzieren. Die Eindämmung und Bewältigung der HIV/AIDS-Epidemie hat allerdings eine eindeutige Priorität beispielsweise in einer südafrikanischen Strategie auf dem Gebiet der öffentlichen Gesundheit. ${ }^{72}$ Dies soll allerdings nicht heißen, dass der Wert von Allgemeinen Bemerkungen des UN-Fachausschusses zum Sozialpakt als Richtlinien für die Umsetzung von universellen Kernverpflichtungen auf nationaler Ebene gering ist. Seine Erfahrung mit der Prüfung von Staatenberichten gibt dem Ausschuss gleichwohl einen weitreichenden Überblick über staatliche Umsetzungsmaßnahmen des Rechts auf Gesundheit in unterschiedlichen Zusammenhängen und Situationen, einschließlich der Umsetzung von Kernverpflichtungen. ${ }^{73}$ Dies versetzt den Ausschuss in die Lage, vergleichende Schlüsse aus der Staatenpraxis zum möglichen universellen Kerngehalt des Rechts auf Gesundheit zu ziehen, die in die Definiti-

folgerungen XIII-4, Statement of Interpretation on Article 13, 54-57; Schlußfolgerungen XIII-2, Griechenland, 181 und Schlußfolgerungen XIII-4, 180-181.

71 Dies spiegelt sich in der »Rechtsprechung " des ECSR wider, z.B. in den Schlußfolgerungen XV-2, Belgien, 99, zum Recht auf gesundheitliche Aufklärung; und Beschwerde 15/2003, European Roma Rights Centre (ERRC) v. Greece, Entscheidung vom 8. Dezember 2003, zum Recht auf eine angemessene Unterkunft, in der vom ECSR angemerkt wird, dass »the implementation of Article 16 [ESC/RESC] as regards nomadic groups including itinerant Roma, implies that adequate stopping places be provided« (para. 25). Der Ausschuss hebt hervor, dass Unterschiede in der Lebensweise von Einzelpersonen und Gruppen angemessen berücksichtigt werden sollen, wenn Maßnahmen zur Umsetzung der ESC/RESC entwickelt werden (para. 21).

72 Vgl. Pillay (2002), 68.

73 Mehr zu Staaten, die einen nationalen Kernbereich der WSK-Rechte definiert haben, siehe Fußnoten 91-98. 
on in den Allgemeinen Bemerkungen Nr. 14 eingeflossen sind. ${ }^{74}$ Die Tatsache, dass die Definition des universellen Kerngehaltes des Rechts auf Gesundheit in den Allgemeinen Bemerkungen Nr. 14 u.a. auf Staatenpraxis beruht, erhöht die praktische Relevanz des universell definierten Kerngehalts als eine Leitlinie für die Umsetzung des Kernrechts auf Gesundheit in bestimmten nationalen Umständen. ${ }^{75}$

Unter Ausklammerung der sog. »zugrundeliegenden Determinanten der Gesundheit« ist der Zugang zu folgenden primären Gesundheitsleistungen in den Allgemeinen Bemerkungen Nr. 14 und der Alma-Ata Deklaration Teil des universell definierten Kernbereichs des Rechts auf Gesundheit und dazugehöriger Kernverpflichtungen: ${ }^{76}$

- Bereitstellung von reproduktiven, (prä- und postnatalen) Mütter- und Kindergesundheitsdiensten,

- Bereitstellung von Impfungen gegen in der Gemeinschaft besonders häufig auftretende Infektionskrankheiten,

- Ergreifen von Maßnahmen zur Prävention, Kontrolle und Behandlung von epidemischen und endemischen Krankheiten,

- Bereitstellung von unentbehrlichen Arzneimitteln, wie sie vom WHO Aktionsprogramm für unentbehrliche Arzneimittel von Zeit zu Zeit definiert werden sowie

- Bereitstellung von gesundheitlicher Aufklärung und Zugang zu Informationen über dominierende Gesundheitsprobleme in der Gemeinschaft, einschließlich der Methoden zur ihrer Prävention und Kontrolle.

Des Weiteren kann ein universell definierter Kerngehalt des Rechts auf Gesundheit potenziell die »internationale Gemeinschaft« und »Staaten, die

74 Dies erkennen auch die Staaten an, siehe Report of the Open-ended Working Group to Consider Options Regarding the Elaboration of an Optional Protocol to the ICESCR on its Third Session, E/CN.4/2006/47, 14. März 2006, para. 91.

75 Siehe unten (Teil 4.2), in dem genauer analysiert wird, wie ein universell definierter Kerngehalt zur Konkretisierung und Umsetzung eines national definierten Kerngehalts des Rechts auf Gesundheit beitragen könnte.

76 E/CN.4/2006/47 paras. 43 und 44 sowie World Health Organization (1978), VII (3). 
in der Position sind zu helfen ${ }^{77}$ anleiten, ab wann ihre internationalen Verantwortlichkeiten, Hilfe zur Umsetzung des Kernrechts auf Gesundheit außerhalb ihrer Grenzen zu leisten, wirksam werden: nämlich dann, wenn der universelle Kerngehalt des Rechts auf Gesundheit wie vom UN-Sozialausschuss definiert einer »beträchtlichen Anzahl von Personen ${ }^{78}$ in einem einkommensschwachen Land nicht umgesetzt ist. ${ }^{79}$ In dieser Rolle macht der universell definierte Kerngehalt die Umsetzung des Rechts auf Gesundheit realistischer für einkommensschwache Staaten, wenn diese nicht über genügend Ressourcen verfügen, um ihre Kernverpflichtungen aus dem Kernrecht aus Gesundheit und anderen WSK-Rechten, wie sie vom UNSozialausschuss definiert sind, umzusetzen. ${ }^{80}$ Außerdem wird damit anerkannt, dass einige Probleme mit der Umsetzung von Kernverpflichtungen nicht nur mit der Ressourcenzuordnung innerhalb eines Staates zusammenhängen, sondern auch mit der globalen Ressourcenverteilung unter Staaten. Dies ist die bereits genannte Funktion des universell definierten Kerngehalts, in der der Kerngehalt dazu beitragen könnte, nationale Verpflichtungen und internationale Verantwortlichkeiten für die Umsetzung des Kernrechts auf Gesundheit und andere WSK-Kernrechte voneinander abzugrenzen und zu koordinieren.

Allerdings beantworten die universellen Kerngehalte der WSK-Rechte, wie vom UN-Sozialausschuss in seinen Allgemeinen Bemerkungen definiert, nicht alle offenen Fragen über den Umfang internationaler Verantwortlichkeit, Hilfe zur Umsetzung von Kernrechten in anderen Ländern zu leisten. Es ist weder klar, wo genau nationale Verpflichtungen aufhören und wo internationale Verantwortlichkeiten beginnen, noch sind bisher eindeutige Kriterien entwickelt worden, die einkommensstarke Länder oder internationale Organisationen anwenden können, wenn sie entscheiden sollen, welches einkommensschwache Land sie unterstützen. ${ }^{81}$ Wenn ein bewaff-

77 Siehe E/C.12/2001/10, 10. Mai 2001, para. 16.

78 Ebd., para. 17 und E/1991/23, 14. Dezember 1990, para. 10.

79 Diese Rolle des Kerngehalts wird auch in den Maastricht Principles on the Extraterritorial Obligations of States in the Area of Economic, Social and Cultural Rights (sog. »Maastricht-Prinzipien«) aus dem Jahr 2011 betont (principle 32).

80 Siehe auch die Analyse von Ssenjonyo (2009), 77-81 und Riedel (2007).

81 Etwas mehr ins Detail zu diesen Fragen gehen International Council on Human Rights Policy (2003) und Ssenyonjo (2009), 80. 
neter Konflikt oder eine Naturkatastrophe eine Massenvertreibung hervorrufen, mag es klar sein, dass die internationale Verantwortlichkeit, Hilfe zu leisten, wirksam wird. In stabileren Situationen bleibt allerdings die Frage unbeantwortet, wann genau eine »beträchtliche Anzahl von Personen« keinen Zugang zu gesundheitlicher Grundversorgung hat, sodass internationale Verantwortlichkeiten relevant werden. Es ist des Weiteren schwer auszumachen, welche internationalen Verantwortlichkeiten welcher einkommensstarken Staaten und internationaler Organisationen in einer bestimmten Situation relevant sind und wie die Umsetzung dieser Verantwortlichkeiten mit der Umsetzung nationaler Verpflichtungen des jeweiligen Vertragsstaates koordiniert werden sollen. ${ }^{82}$

\subsection{Der national definierte Kerngehalt des Rechts auf Gesundheit}

Im rechten Kasten der Abbildung 1 sind die Eigenschaften des national definierten Kernbereichs des Rechts auf Gesundheit und dazugehöriger Kernverpflichtungen aufgelistet. Wegen der beträchtlichen Unterschiede zwischen Staaten und der damit verbundenen Unmöglichkeit, den Inhalt des Kernrechts auf Gesundheit und dazugehörige Kernverpflichtungen im Detail auf internationaler Ebene festzulegen, ist es wichtig, dass Staaten einen pragmatischen und detaillierten materiellen Kerngehalt des Rechts auf Gesundheit auf nationaler Ebene festlegen, unter Berücksichtigung nationaler Besonderheiten, aber geleitet vom universell definierten Kerngehalt des Rechts auf Gesundheit. Ein national definierter Kerngehalt kann besser auf die nationalen Merkmale eines bestimmten Landes eingehen, besonders wenn detaillierte Maßnahmen und Strategien entwickelt werden, um Kernverpflichtungen aus dem Recht auf Gesundheit auf nationaler Ebene umzusetzen. ${ }^{83}$ Unter anderem können der Inhalt und die Struktur nationaler Ver-

82 Die Maastricht-Prinzipien greifen diese Fragen zu einem gewissen Grad auf (principles 30-35). Siehe auch Forman et al. (2013), 7-9 und die Beträge in Langford et al. (2014).

83 Wie vom UN-Sozialausschuss und dem ECSR anerkannt. Siehe z.B. Artikel 8 (4) des Zusatzprotokolls zum IPwskR, der festhält, dass »[...] the Committee shall bear in mind that the State Party may adopt a range of possible policy measures for the implementation of the rights set forth in the Covenant«. Ge- 
fassungen und die Verfügbarkeit von finanziellen Mitteln und fachlichen Kapazitäten bei der Konkretisierung des national definierten Kerngehalts berücksichtigt werden. Pieterse schreibt beispielsweise, dass, wenn Südafrika einen nationalen Kerngehalt des Rechts auf Gesundheit bestimmen würde, dieser nicht die sog. nicht-medizinischen Determinanten von Gesundheit einschließen würde, obwohl dies vom UN-Sozialausschuss in den Allgemeinen Bemerkungen Nr. 14 geraten wird, da diese von anderen WSK-Rechten in der südafrikanischen Verfassung abgedeckt werden. ${ }^{84}$ Dennoch sollte der konkrete Kerngehalt eines national-definierten Rechts auf Gesundheit vom international definierten Kerngehalt geleitet werden, wenn die konkreten Gesundheitsleistungen bestimmt werden, zu denen jeder, der sich unter der Hoheitsgewalt des jeweiligen Vertragsstaates befindet, unmittelbar Zugang haben sollte. Im Lichte des universell definierten Kerngehalts, wie er oben beschrieben wurde, muss zum Beispiel definiert werden, welche Infektionskrankheiten lokal besonders verbreitet sind und welche Impfungen bereitgestellt werden müssen; welche die häufigsten epidemischen und endemischen Krankheiten in einem bestimmten Land sind, zu deren Prävention, Kontrolle und Behandlung prioritär Maßnahmen ergriffen werden sollten, und welche unentbehrlichen Medikamente jedem in einem bestimmten Land zur Verfügung gestellt werden sollten. ${ }^{85}$

nauso er kennt die »Rechtsprechung « des ECSR einen Ermessensspielraum der Vertragsstaaten in der Umsetzung der Verpflichtungen aus der ESC/RESC an. Betreffs Artikel 12 (3) ESC hat der ECSR z.B. festgestellt: »States enjoy a wide margin of discretion on how to organize their social security systems, including defining the personal scope of schemes providing health care benefits, as long as a significant percentage of the population is covered and the benefits provided are sufficiently extensive« (ECSR, Beschwerde 43/2007, Sindicato dos Magistrados do Ministério Publico (SMMP) v. Portugal, Entscheidung vom 3. Dezember 2008, para. 44); siehe auch Beschwerde 30/2005, Marangopoulos Foundation for Human Rights (MFHR) v. Greece, Entscheidung vom 6. Dezember 2006, para. 221 und Beschwerde 31/2005, European Roma Rights Centre (ERRC) v. Bulgaria, Entscheidung vom 18. Oktober 2006, paras. 35-54.

84 Pieterse (2006), 490-491.

85 Siehe z.B. UN-Sozialausschuss, Abschließende Bemerkungen - Russland, E/C.12/1/Add.13, 20. Mai 1997, in welchen der Ausschuss Russland dazu aufruft, eines der Hauptprobleme der öffentlichen Gesundheit, die achtfache Zu- 
Der universell definierte Kerngehalt des Rechts auf Gesundheit kann verhindern, dass der national definierte Kerngehalt willkürlich festgelegt wird. Er kann die nationale Prioritätensetzung bei der Umsetzung des Rechts auf Gesundheit durch die Einführung gesetzlicher und anderer Maßnahmen so beeinflussen, dass diese Maßnahmen einem Großteil der Bevölkerung zu Gute kommen ${ }^{86}$ und besonders die Interessen derer berücksichtigt, die die dringendsten grundlegenden Gesundheitsbedürfnisse haben. ${ }^{87}$ Wenn der Zugang zu Gesundheitsleistungen, der aus dem national definierten Kerngehalt des Rechts auf Gesundheit gewährt wird, unterhalb der Forderungen des universell definierten Kerngehalts liegt, ist dies ein Zeichen, dass die Staaten vorrangig und zielstrebig ihre Bemühungen intensivieren müssen, den national definierten Kerngehalt des Rechts auf Gesundheit auf den des universell definierten Kerngehaltes anzuheben. ${ }^{88}$ Diese Vorrangigkeit wird von der Tatsache bekräftigt, dass sich der universelle Kernbereich des Rechts auf Gesundheit und anderer WSK-Rechte auf menschliche Überlebensinteressen und fundamentale Grundbedürfnisse stützt. Wenn der national definierte Kerngehalt in einem Vertragsstaat wegen mangelnder Ressourcen oder aus anderen Gründen unterhalb des universell definierten Kerngehalt liegt, sind die betreffenden staatlichen Behörden besonders dazu angehalten, internationale Organisationen und Drittstaaten um Hilfe und Unterstützung zu bitten. Dies hat der UN-Sozialausschuss in einigen Aussagen bekräftigt. ${ }^{89}$

nahme an HIV-Infektionen 1996, als eine »health question of the utmost importance« (para. 40) anzugehen.

86 Dies beruht auf der obengenannten Annahme, dass ein universell definierter Kerngehalt Rechte auf Zugang zu Gesundheitsleistungen gibt, welche die am weitesten verbreiteten Gesundheitsprobleme in einer Gemeinschaft angehen und deshalb der großen Mehrheit der Bevölkerung zu Gute kommen.

87 Die Verpflichtung, besondere Rücksicht auf die Interessen von sozioökonomisch benachteiligten Einzelpersonen und Gruppen zu nehmen, wird weiter unten (Teil 4.3.) noch etwas ausführlicher diskutiert.

88 Wie vom UN-Sozialausschuss vorgeschlagen, siehe Fußnoten 52-55. Wie dies für den südafrikanischen Kontext für das Recht auf soziale Sicherheit in der Praxis funktionieren könnte, diskutiert Liebenberg (2006), 32-33.

89 Siehe z.B. E/C.12/2007/1, 21. September 2007, paras. 5 und 10 (f) sowie die Maastricht-Prinzipien, principle 34. 
Vergleichende Studien nationaler und internationaler Rechtsprechung zu WSK-Rechten zeigen, dass einige Länder tatsächlich konkrete nationale Kerngehalte unterschiedlicher WSK-Rechte formulieren, welche vor nationalen Gerichten direkt geltend gemacht werden können. ${ }^{90}$ Um nur einige wenige, nicht auf das Recht auf Gesundheit begrenzte Beispiele zu nennen: Das kolumbianische Verfassungsgericht hat beispielsweise »Minimalbedingungen für ein menschenwürdiges Leben« entwickelt, die auf den Rechten auf Leben, Gesundheit, Arbeit und soziale Sicherheit der kolumbianischen Verfassung aufbauen. ${ }^{91}$ Wenn staatliche Sozialpolitik diese Minimalbedingungen nicht erfüllt, greifen nationale Gerichte ein und ordnen die sofortige Umsetzung relevanter Kernrechte an, selbst wenn dies dazu führt, dass einer bestimmten Person Zugang zu bestimmten Leistungen, Gütern oder Programmen gegeben werden muss, und selbst wenn dies Auswirkungen auf die Zuteilung finanzieller Ressourcen hat. ${ }^{92}$ Ähnliche Rechtsprechung kommt u.a. von nationalen Gerichten in Argentinien, ${ }^{93}$ Brasilien, ${ }^{94}$ Deutschland, ${ }^{95}$ Finnland, ${ }^{96}$ Indien ${ }^{97}$ und Portugal. ${ }^{98}$

90 Langford (2008), 22 stellt fest: »in broad brush terms, many adjudicators tend to enforce one or both of the two key state obligations identified by the CESCR: First, the adequate duty to take steps towards the progressive realisation of the rights within available resources and, secondly, the implicit obligation to immediately achieve a minimum level of realisation«. Siehe auch O’Cinneide (2014), 175.

91 Vgl. Langford (2008), 22 und Sepulveda (2008), 147-148.

92 Sepulveda (2008) analysiert die Rechtsprechung des kolumbianischen Verfassungsgerichts gründlich. In Bezug auf das Recht auf Gesundheit hat das kolumbianische Verfassungsgericht beispielsweise schon häufig angeordnet, dass staatliche oder private Einrichtungen Einzelpersonen (besonders Kinder) unmittelbar mit bestimmten Medikamenten oder einer medizinischen Behandlung versorgen müssen, um das Recht auf Gesundheit zu wahren. Wie Sepulveda des Weiteren feststellt, unterscheidet sich der vom kolumbianischen Verfassungsgericht gewählte Ansatz damit von dem des südafrikanischen Verfassungsgerichts in Soobramoney v. Minister of Health (Kwazulu-Natal), Entscheidung vom 27. November 1997, [1997] ZACC 17. Das südafrikanische Verfassungsgericht lehnt den Kerngehaltsansatz ab.

93 Der argentinische Supreme Court hat beispielsweise festgestellt: »in light of the human right to health guaranteed by the [Argentine] Constitution and 
Des Weiteren muss anerkannt werden, dass der materielle, national definierte Kerngehalt des Rechts auf Gesundheit und die dazugehörigen Verpflichtungen dynamisch sind, d.h. dass ihr Umfang und Inhalt Änderungen unterworfen sein kann. Inhalt und Umfang ändern sich im Lichte wirtschaftlicher, sozialer, technischer, medizinischer und anderer Entwicklungen, die beispielsweise die wesentlichen Gesundheitsprobleme, die ein bestimmtes Land berühren, verändern können. ${ }^{99}$ Dies macht eine regelmäßige Anpassung des national definierten Kerngehalts nötig. Bruce Porter hat außerdem darauf hingewiesen, dass es möglich sein sollte, Forderungen unter dem Recht auf Gesundheit ausreichend an individuelle Erfahrungen und Gesundheitsinteressen zu koppeln. ${ }^{100}$ Obwohl national definierte Kerngehalte und Kernverpflichtungen spezifisch sein sollten, dürfen sie deshalb nicht $\mathrm{zu}$ rigide und unflexibel sein, sodass ihre Anwendung auf individuelle Situationen ermöglicht wird. Die Möglichkeiten einer individuellen An-

international human rights treaties, statutory regulations granting access to medical services should be read as requiring health care givers to fully provide essential medical services in case of need «. Zitiert in International Commission of Jurists (2008), 25; siehe auch Courtis (2008), 163-181.

94 Vgl. International Commission of Jurists (2008), 25.

95 Siehe insbesondere die Rechtsprechung zum menschenwürdigen Existenzminimum: BVerfG, 1 BvL 1/09 vom 9. Februar 2010 (»Hartz-IV «) und 1 BvL 10/10 vom 18. Juli 2012 (»Asylbewerberleistungsgesetz«). Die Rechtsprechung des Bundesverfassungsgerichts wird auch von der International Commission of Jurists (2008), 24 und O’Cinneide (2014), 175-176 analysiert.

96 Scheinin (2001), 51-53.

97 Siehe z.B. Supreme Court of India (1996), der festgestellt hat, dass der Zugang zu notfallmedizinischer Behandlung Teil des Kernrechts auf Gesundheit in Indien ist. Dazu auch die Analyse von Muralidhar (2008), 117-118.

98 O’Cinneide (2014), 176.

99 Wie der UN-Sozialauschuss in den Allgemeinen Bemerkungen Nr. 14 (E/C.12/2000/4, 11. August 2000) feststellt, para. 43 (f), und wie es in der »Rechtsprechung « des ECSR angedeutet ist, siehe z.B. Schlußfolgerungen I, Statement of Interpretation on Article 1(1), 13; Schlußfolgerungen XIII-5, Statement of Interpretation on Article 23, 455 und Schlußfolgerungen XV-2, Belgien, 99.

100 Porter (2005), 52. 
wendung und dynamischen Interpretation des nationalen Kernrechts auf Gesundheit, die sich ändernde Umstände berücksichtigen kann, werden erhöht, wenn der national definierte Kerngehalt vor nationalen Gerichten geltend gemacht werden kann. Nationale Gerichte können dann von Fall zu Fall entscheiden, ob die nationale Konkretisierung des Kerngehalts durch die Legislative genügt, Einzelpersonen Zugang zu relevanter medizinischer Grundversorgung zu verschaffen. ${ }^{101}$ Generell wird eine klare Definition der primären Gesundheitsleistungen, zu denen Einzelpersonen in einem bestimmten Vertragsstaat unter ihrem national bestimmten Kernrecht auf Gesundheit Zugang haben sollten, die Justiziabilität und Möglichkeiten der gerichtlichen Durchsetzung des Kernrechts auf Gesundheit erhöhen.

\subsection{Die Wichtigkeit verfahrensbezogener Rechte und Verpflichtungen}

Verfahrensbezogene Verpflichtungen sind im unteren Kasten von Abbildung 1 zusammengefasst. Es ist wichtig, dass Staaten diese Verpflichtungen im Konkretisierungsprozess ihres national definierten, materiellen Kerngehalts des Rechts auf Gesundheit befolgen. Man kann argumentieren, dass diese verfahrensbezogenen Verpflichtungen Teil der universell definierten Kernverpflichtungen aus dem Recht auf Gesundheit und anderer WSK-Rechte sind, die die Staaten in der Bestimmung ihres detaillierten, nationalen Kerngehalts leiten sollten.

Wie in den Allgemeinen Bemerkungen Nr. 14 und anderen Allgemeinen Bemerkungen des UN-Sozialausschusses dargelegt, ist zunächst »the participation of the population in all health-related decision-making at the community, national and international levels « ${ }^{102}$ eine wichtige verfahrensbe-

101 Die BVerfG »Hartz-IV «-Entscheidung ist ein gutes Beispiel in diesem Kontext. Sie hebt u.a. hervor, dass die besonderen Lebensumstände und Interessen unterschiedlicher Gruppen (in diesem Fall von Kindern) berücksichtigt werden müssen, wenn die Höhe von Sozialleistungen bestimmt wird. Siehe auch Pieterse (2006), 491 und Bilchitz (2007), 224-225.

102 E/C.12/2000/4, 11. August 2000, para.11. Die Wichtigkeit der Beteiligung in gesundheitspolitischen Entscheidungen wir auch vom ECSR anerkannt, siehe z.B. Marangopoulos Foundation for Human Rights (MFHR) v. Greece, Beschwerde 30/2005, Entscheidung vom 6. Dezember 2006, paras. 216 und 219. 
zogene Verpflichtung unter dem Recht auf Gesundheit und anderen WSK-Rechten. Dies ist sowohl mit der Annahme verknüpft, dass Menschenrechte am besten in einem demokratischen System geschützt werden können - eine Annahme, die sich aus den Bezugnahmen des IPwskR auf eine »demokratische Gesellschaft « ${ }^{103}$ speist -, als auch mit der Interdependenz von WSK-Rechten und zivilen und politischen Rechten, wie sie sich explizit im Wortlaut des Artikel 6 (2) IPwskR offenbart. ${ }^{104}$ Dies bedeutet, dass Staaten einen Mechanismus schaffen sollten, durch den die »Teilnahme an politischen Entscheidungen auf kommunaler und nationaler Ebene, die das Recht auf Gesundheit betreffen «, ${ }^{105}$ ermöglicht wird. Dies sollte Entscheidungen über die konkreten Gesundheitsleistungen einschließen, zu welchen jeder unmittelbaren und direkten Zugang unter dem national definierten Kernrecht auf Gesundheit haben sollte. Für gewöhnlich hat dieser Mechanismus die Form eines gewählten Parlaments - eine Tatsache, welche die starke Verbindung zwischen WSK-Rechten und bürgerlichen Rechten noch einmal hervorhebt. Die Tatsache, dass Entscheidungen über den Inhalt des national definierten Kerngehalts in einkommensschwachen Ländern zum Teil auf utilitaristischen Erwägungen beruhen, was tragische Konsequenzen für Einzelpersonen haben kann, deren spezielle gesundheitliche Probleme nicht als Teil der Kerngesundheitsleistungen behandelt

103 Artikel 4, 8 (1) (a) und (c) ICESCR. Generell zum Verhältnis Menschenrechte und Demokratie siehe Besson (2011).

104 Artikel 6 IPwskR: »(1) Die Vertragsstaaten erkennen das Recht auf Arbeit an, welches das Recht jedes einzelnen auf die Möglichkeit, seinen Lebensunterhalt durch frei gewählte oder angenommene Arbeit zu verdienen, umfasst, und unternehmen geeignete Schritte zum Schutz dieses Rechts. (2) Die von einem Vertragsstaat zur vollen Verwirklichung dieses Rechts zu unternehmenden Schritte umfassen fachliche und berufliche Beratung und Ausbildungsprogramme sowie die Festlegung von Grundsätzen und Verfahren zur Erzielung einer stetigen wirtschaftlichen, sozialen und kulturellen Entwicklung und einer produktiven Vollbeschäftigung unter Bedingungen, welche die politischen [Hervorhebung A.M.] und wirtschaftlichen Grundfreiheiten des einzelnen schützen.«

105 E/C.12/2000/4, 11. August 2000, para. 17 [Übersetzung A.M.]. 
werden können, ${ }^{106}$ macht einen demokratisch-partizipatorischen Entscheidungsprozess umso wichtiger. In Situationen, in denen Ressourcen begrenzt sind und in denen deshalb moralisch schwierige Entscheidungen getroffen werden müssen, könnte ein solcher Prozess zumindest ein Stück weit zur Legitimierung der Priorisierung einiger Gesundheitsleistungen über andere beitragen, besonders wenn der international definierte Kerngehalt des Rechts auf Gesundheit als Richtlinie dient, der eine Priorisierung von primären Gesundheitsleistungen fördert, die das Überlebensinteresse der großen Mehrheit der Menschen wahren kann. Weiter legitimiert würde die Priorisierung durch die Erarbeitung eines Plans zur schrittweisen Ausweitung der Gesundheitsleistungen (einschließlich Sekundär- und Tertiärversorgung), zu denen jeder unmittelbar Zugang haben sollte, im Einklang mit der Verpflichtung auch Nicht-Kernbereiche des Rechts auf Gesundheit nach und nach umzusetzen.

Verbunden mit der verfahrensbezogenen Verpflichtung, effektive Mitwirkung in gesundheitspolitischen Entscheidungen zu ermöglichen, ist die weitere verfahrensbezogene Verpflichtung, den Gesundheitsbedürfnissen sog. marginalisierter Bevölkerungsgruppen und vulnerabler Einzelpersonen besondere Aufmerksamkeit in der Bestimmung des konkreten Inhalts des nationalen Kernrechts auf Gesundheit zu schenken. ${ }^{107}$ Dies könnte bedeuten, dass nationale Parlamente solche Gruppen direkt in Konsultationen mit einbeziehen sollten, wenn der konkrete Inhalt des nationalen Kernrechts auf Gesundheit bestimmt wird. Sogar in gut ausgebauten demokratischen Systemen kann es sein, dass sozioökonomisch benachteiligte Gruppen weniger gut im Parlament repräsentiert sind. Um die Wichtigkeit der verfahrensbezogenen Verpflichtung zur politischen Mitwirkung bei Gesundheitsentscheidungen hervorzuheben, bemerkt der UN-Sozialausschuss, dass »die effektive Bereitstellung von Gesundheitsleistungen nur dann sichergestellt

106 Diese Problematik offenbart sich in der sog. Soobramoney Entscheidung des südafrikanischen Verfassungsgerichts (Fußnote 92); siehe auch Lehmann (2006), 90.

107 E/C.12/2000/4, 11. August 2000, paras. 43 (a) und (f). Wenn auch nur in generellen Zügen, erkennt der ECSR gleichfalls die verfahrensbezogene Verpflichtung an, dass den Interessen benachteiligter Gruppen besonderes Gehör verschafft werden sollte, siehe z.B. Schlußfolgerungen 2005, Statement of Interpretation on Article 11 (5), 10 und Schlußfolgerungen XVII-2, Portugal, 681. 
werden kann, wenn Staaten die Beteiligung der Bevölkerung in Entscheidungsprozessen ermöglichen ${ }^{108}$ - eine Mitwirkung, die idealer Weise so viele Einzelpersonen und Gruppen wie möglich mit einschließen sollte.

Zweitens haben der Sozialausschuss und nationale Gerichte festgestellt, dass Entscheidungsprozesse über den Kernumfang des Zugangs zu Gesundheits- und anderen Sozialleistungen transparent sein müssen und sich auf verlässliche Daten stützen sollen. Das deutsche Bundesverfassungsgericht hat beispielsweise detaillierte verfahrensbezogene Verpflichtungen des Parlaments formuliert, die befolgt werden müssen, wenn die Höhe von (Kern-)Sozialleistungen bestimmt wird, die jedem Empfänger ein menschenwürdiges Existenzminimum sichern sollen: Die Höhe muss durch einen objektiven und transparenten Prozess bestimmt werden, auf verlässlichen Daten basieren sowie einer verständlichen und realistischen Berechnungsmethode folgen. ${ }^{109}$ In gleicher Weise könnten solche verfahrensbezogenen Verpflichtungen für die konkrete Bestimmung nationaler Kernbereiche des Rechts auf Gesundheit relevant sein. Im Kontext des Rechts auf Gesundheit könnte eine solche Verpflichtung implizieren, dass Experten für öffentliche Gesundheit konsultiert werden, die anhand von epidemiologisch ermittelten Daten a) verbindliche Impfempfehlungen für impfpräventive Infektionskrankheiten in dem jeweiligen Land abgeben; b) prioritäre Maßnahmen zur Prävention, Kontrolle und Behandlung häufiger Erkrankungen vorschlagen; wasc) eine Liste unentbehrlicher Arzneimittel, zu denen jeder Zugang haben sollte, einschließt. ${ }^{110}$ Wichtig ist auch, dass die Mitwirkung im Konkretisierungsprozess des national definierten Kernrechts auf Gesundheit nicht diskriminierend ist und dass auch im Zugang zu Gesundheitsleistungen unter dem Kernrecht nicht diskriminiert wird. ${ }^{111}$

Der Sozialpakt und die Allgemeinen Bemerkungen Nr. 14 deuten zudem darauf hin, dass die konkreten grundlegenden Gesundheitsleistungen, zu denen jeder unter einem national definierten Kernrecht auf Gesundheit Zugang haben sollte, im nationalen Recht festgelegt werden sollten. Diese

108 E/C.12/2000/4, 11. August 2000, para. 54 [Übersetzung A.M.].

109 Siehe die Rechtsprechung zum menschenwürdigen Existenzminimum zitiert in Fußnote 95.

110 E/C.12/2000/4, 11. August 2000, para. 56, deutet an, dass eine Rolle für Experten der öffentlichen Gesundheit in diesem Prozess vorgesehen ist.

111 Ebd., paras. 12 (b) und (i), 18-20 und 43 (e). 
weitere verfahrensbezogene Verpflichtung ist besonders klar aus dem Wortlaut von Artikel 2 (1) IPwskR abzuleiten, der sich auf das Ergreifen "gesetzgeberischer Maßnahmen« zur schrittweisen Umsetzung des Rechts auf Gesundheit und anderer WSK-Rechte bezieht. Dass die Legislative direkt im Vertragstext angesprochen ist hebt diese verfahrensbezogene Verpflichtung ebenfalls hervor.

Als letztes muss eine weitere verfahrensbezogene Verpflichtung genannt werden, deren Analyse und Diskussion in Lehre und Praxis gerade erst begonnen hat und die sich an nationale Parlamente und andere nationale Institutionen richtet, die die Funktion haben zu bestimmen, welche Gesundheitsleistungen unter dem national definierten Kernrecht auf Gesundheit bereitgestellt werden sollen: Es handelt sich um die Verpflichtung die Kernverpflichtungen aus dem Recht auf Gesundheit klar den verschiedenen Akteuren, die an der Umsetzung beteiligt sind, zuzuordnen und diese Umsetzung zu koordinieren. Wenn beispielsweise private Akteure an der Bereitstellung von medizinischer Grundversorgung beteiligt sind, müssen deren Kernverpflichtungen genau definiert und zugeordnet sowie ihre Umsetzung von staatlichen Stellen koordiniert und überwacht werden; ${ }^{112}$ dasselbe gilt für staatliche Gesundheitseinrichtungen, z.B. Krankenhäuser, Apotheken, Rehabilitationszentren etc. ${ }^{113}$ Für einkommensschwache Staaten würden diese Zuordnungs- und Koordinationspflichten wohl auch eine Verpflichtung beinhalten, Drittstaaten (und internationale Organisationen) darauf hinzuweisen, in welchem Gesundheitsbereich diese Staaten (und Organisationen) am besten ihrer internationalen Verantwortlichkeit, zur Umsetzung des Kernrechts auf Gesundheit außerhalb ihrer eigenen Landesgrenzen beizutragen, nachkommen sollten. Diese Verpflichtung bleibt allerdings unspezifisch und problematisch in der Umsetzung. Wie bereits angemerkt, bleibt es unklar, wann genau internationale Verantwortlichkeiten für die Umsetzung des Kernrechts auf Gesundheit in einem bestimmten, einkommensschwachen Staat wirksam werden und welcher Drittstaat oder welche internationale Organisation genau von diesen Verantwortlichkeiten in einer bestimmten Situation »angesprochen« ist. Offene Fragen bleiben auch, inwieweit ein Vertragsstaat Drittstaaten und internationale Organisa-

112 E/C.12/2000/4, 11. August 2000, paras. 26 (in Bezug auf Personen mit Behinderung) und 55; und generell para. 51.

113 Dies wird auch vom ECSR anerkannt, siehe Schlußfolgerungen 2006, 9-10. 
tionen anleiten kann, wie sie ihre internationalen Verantwortlichkeiten erfüllen sollen, sodass ein vom Vertragsstaat national definierter Kerngehalt mit internationaler Hilfe effektiv implementiert werde kann. Ein solches Anleiten ist besonders problematisch, wenn der Konkretisierungsprozess des nationalen Kerngehalts nicht demokratisch abgelaufen ist und nicht die drängendsten Gesundheitsinteressen der Mehrheit der Bevölkerung widerspiegelt. Ohne eine klare Zuordnung von nationalen Kernverpflichtungen und internationalen Kernverantwortlichkeiten an nationale und internationale Akteure und ohne die effektive Koordinierung der Umsetzung dieser Kernverpflichtungen und -verantwortlichkeiten ist es allerdings unwahrscheinlich, dass Kernrechte auf Gesundheit in einkommensschwachen Staaten effektiv umgesetzt werden können. ${ }^{114}$ Das ist insbesondere dann der Fall, wenn die jeweiligen einkommensschwachen Staaten nicht über ausreichende Ressourcen und fachliche Kapazitäten verfügen, um beispielsweise unentbehrliche Medikamente herzustellen. ${ }^{115}$

Eine weitere Klarstellung dieser verfahrensbezogenen Verpflichtungen würde außerdem dazu beitragen, die Überprüfungsmöglichkeiten der Umsetzung des Kernrechts auf Gesundheit durch nationale und internationale Gerichte zu stärken. In einigen Situationen könnten nationale und internationale Gerichte es vorziehen, ihre Überprüfung möglicher Verletzungen auf verfahrensbezogene Verpflichtungen zu beschränken, aus Rücksicht auf die Kompetenzen der Exekutive und Legislative und das Gewaltenteilungsprinzip. ${ }^{116}$ Dies soll allerdings nicht heißen, dass nationale Gerichte die Überprüfung materieller Kernverpflichtungen ganz beiseitelassen sollten, da dies der effektiven Durchsetzung von Kernrechten abträglich sein kann. ${ }^{117}$ Internationale Gerichte oder quasi-gerichtliche Einrichtungen wie der UN-Sozialausschuss könnten es manchmal vorziehen, sich auf die

114 Wie erwähnt, befassen sich die Maastricht-Prinzipien mit diesem Problem. Die Prinzipien 28-35 beantworten allerdings nicht alle offenen Fragen im Detail. Siehe auch Forman et al. (2013) und die Beträge in Langford et al. (2014).

115 Für eine ausgezeichnete Diskussion dieser Problematik siehe Besson (2013).

116 Dieses Thema kann hier nicht im Detail diskutiert werden, siehe aber beispielsweise Brems/Lavrysen (2013).

117 Die möglichen Gefahren, die mit einer »Prozeduralisierung « der gerichtlichen Überwachung von Menschenrechten verbunden sind, werden von Brems und Lavrysen (2013) diskutiert (für den Kontext des EGMR). 
Überwachung der Umsetzung von verfahrensbezogenen Kernverpflichtungen aus dem IPwskR zu konzentrieren, weil es, wie oben angemerkt, für sie manchmal schwierig sein kann, das konkrete Ausmaß einer universell bestimmten materiellen Kernverpflichtung aus dem Recht auf Gesundheit im Detail zu bestimmen. ${ }^{118}$

\section{SCHLUSSBEMERKUNG}

Der vorliegende Beitrag skizziert den Kernbereich des Menschenrechts auf Gesundheit und die dazugehörigen Kernverpflichtungen. Es wurde aufgezeigt, dass es entscheidend ist, eine sinnvolle Beziehung zwischen einem notwendigerweise generell formulierten universell bestimmten Kernbereich und einem konkreten, national bestimmten Kernbereich des Rechts auf Gesundheit zu definieren, um wenigstens das Kernrecht auf Gesundheit aller Menschen weltweit effektiv umzusetzen. Eine solche sinnvolle Beziehung würde sicherstellen, dass das Recht auf Gesundheit und andere WSKRechte konkrete, aussagekräftige Inhalte bekommen, die unmittelbar und direkt eingefordert werden können, trotz der »bloßen « Verpflichtung aus dem IPwskR, das Recht auf Gesundheit und andere WSK-Rechte nur "schrittweise« umzusetzen. Gleichzeitig würde eine solche sinnvolle Beziehung anerkennen, dass es unmöglich ist, einen universellen Kerngehalt des Rechts auf Gesundheit und anderer WSK-Rechte und dazugehörige Kernverpflichtungen im Detail auf internationaler Ebene zu definieren. Deshalb ist es notwendig, dass eine Konkretisierung auf nationaler Ebene stattfindet, die nationalen Gegebenheiten Rechnung tragen kann, einschließlich vorhandener Ressourcen und fachlicher Kapazitäten.

Die in diesem Beitrag durchgeführte erste Analyse relevanter Materialien hat gezeigt, dass sich ein sinnvolles Zusammenspiel zwischen einem universell definierten Kerngehalt und einem pragmatischen, national definierten Kerngehalt des Rechts auf Gesundheit durchaus aus den Aussagen des UN-Sozialausschusses und des ECSR sowie aus der Rechtsprechung nationaler Gerichte und des EGMR herauslesen lässt. Des Weiteren wurde die Wichtigkeit verfahrensbezogener Kernverpflichtungen für den Konkre-

118 Für eine Analyse der »Prozeduralisierung« in der Rechtsprechung des EGMR siehe ebd. 
tisierungsprozess eines national bestimmten Kerngehalts des Rechts auf Gesundheit und anderer WSK-Rechte hervorgehoben. Weitere Recherchen zu den Detailaspekten verschiedener nationaler und internationaler sowie materieller und verfahrensbezogener Verpflichtungen bzw. Verantwortlichkeiten, ihr Zusammenspiel, ihre effektive Zuordnung zu nationalen und internationalen Akteuren und die Koordination ihrer Umsetzung in verschiedenen Ländern und Situationen könnten beitragen $\mathrm{zu}$ einer verbesserten Umsetzung des Kernrechts auf Gesundheit eines Jeden, der sich unter der Hoheitsgewalt eines Vertragsstaates des IPwskR befindet.

\section{LITERATUR}

Alston, Philip/Tomaševski, Katarina (Eds.) (1984): The Right to Food, Utrecht: Martinus Nijhoff.

Alston, Philip (1987): „Out of Abyss: The Challenges Confronting the New UN Committee on Economic, Social and Cultural Rights«, in: Human Rights Quarterly 9, 3 (1987), 332-353.

Arambulo, Kitty (1999): Strengthening the Supervision of the International Covenant on Economic, Social and Cultural Rights: Theoretical and Procedural Aspects, Antwerp: Intersentia.

Baderin, Mashood/McCorquodale, Robert (Eds.) (2007): Economic, Social and Cultural Rights in Action, Oxford: Oxford University Press.

Besson, Samantha (2013): »The Allocation of Anti-Poverty Rights Duties Our Rights, but Whose Duties?«, in: Nadakavukaren Schefer (2013), 408-431.

Besson, Samantha (2011): »Human Rights and Democracy in a Global Context: Decoupling and Recoupling«, in: Ethics \& Global Politics 4 (2011), 19-50.

Bilchitz, David (2002): »Giving Socio-Economic Rights Teeth: The Minimum Core and Its Importance«, in: South African Law Journal 118 (2002), 484-501.

Bilchitz, David (2007): Poverty and Fundamental Rights: The Justification and Enforcement of Socio-Economic Rights, Oxford: Oxford University Press. 
Brand, Danie/Russell, Sage (Eds.) (2002): Exploring the Core Content of Economic and Social Rights: South African and International Perspectives, Pretoria: Protea Book House.

Brems, Eva/Lavrysen, Laurens (2013): »Procedural Justice in Human Rights Adjudication: The European Court of Human Rights«, in: Human Rights Quarterly 35, 1 (2013), 176-200.

Chapman, Audrey (2002): „Core Obligations Related to the Right to Health and Their Relevance for South Africa«, in: Brand/Russel (2002), 35-60.

Chapman, Audrey/Russel, Sage (Eds.) (2002): Core Obligations: Building a Framework for Economic, Social and Cultural Rights, Antwerp: Intersentia.

COHRE (2003): „Litigating Economic, Social and Cultural Rights: Achievements, Challenges and Strategies - Featuring 21 Case Studies«, Online: www.globalinitiative-escr.org/wp-content/uploads/2012/06/Liti gating-ESCR-Report.pdf [06.01.2016].

Courtis, Christian (2008): »Argentina - Some Promising Signs«, in: Langford (2008), 163-172.

Clements, Luke/Simmons, Alan (2008): »European Court of Human Rights - Sympathetic Unease«, in: Langford (2008), 409-427.

Craven, Matthew (1995): The International Covenant on Economic, Social and Cultural Rights: A Perspective on its Development, Oxford: Clarendon Press.

De Vos, Pierre (2002): »The Essential Components of the Human Right to Adequate Housing - A South African Perspective«, in: Brand/Russel (2002), 23-34.

Eide, Asbjørn/Krause, Catarina/Rosas, Allan (Eds.) (2001): Economic, Social and Cultural Rights. A Textbook, Dordrecht: Martinus Nijhoff.

Forman, Lisa/Ooms, Gorik/Chapman, Audrey/Friedman, Eric/Waris, Attiya/Lamprea, Everaldo/Mulumba, Moses (2013): „What Could a Strengthened Right to Health Bring to the Post-2015 Health Development Agenda? Interrogating the Role of the Minimum Core Concept in Advancing Essential Global Health Needs«, in: BMC International Health and Human Rights 13 (2013), 1-11.

Harris, David/O’Boyle, Michael/Bates, Edward/Buckley, Carla (2009): Law of the European Convention on Human Rights, Oxford: Oxford University Press. 
International Commission of Jurists (2008): „Courts and the Legal Enforcement of Economic, Social and Cultural Rights - Comparative Experience of Justiciability«, in: Human Rights and Rule of Law Series No. 2, Online: www.refworld.org/docid/4a7840562.html [01.01.2016].

International Council on Human Rights Policy (2003): »Duties sans Frontiers - Human Rights and Global Social Justice«, Online: www.ichrp.org/files/reports/43/108_report_en.pdf [01.01.2016].

Kende, Mark (2004): »The South African Constitutional Court's Construction of Socio-Economic Rights: A Response to Critics«, in: Connecticut Journal of International Law 19 (2004), 617-629.

Koch, Ida Elisabeth (2009): Human Rights as Indivisible Rights: The Protection of Socio-Economic Demands under the European Convention on Human Rights, Leiden: Martinus Nijhoff.

Künnemann, Rolf (2002): »The Right to Adequate Food: Violations

Related to its Minimum Core Content«, in: Chapman/Sage (2002), 161-183.

Langford, Malcolm (Ed.) (2008): Social Rights Jurisprudence: Emerging Trends in International and Comparative Law, Cambridge: Cambridge University Press.

Langford, Malcolm/Vandenhole, Wouter/Scheinin, Martin van Genugten, Willem (Eds.) (2014): Global Justice, State Duties - The Extraterritorial Scope of Economic, Social and Cultural Rights in International Law, Cambridge: Cambridge University Press.

Lehmann, Karin (2006): »In Defense of the Constitutional Court: Litigating Socio-Economic Rights and the Myth of the Minimum Core«, in: American University International Law Review 22 (2006), 163-197.

Liebenberg, Sandra (2002): „South Africa’s Evolving Jurisprudence on Socio-Economic Rights: An Effective Tool in Challenging Poverty«, in: Law, Democracy and Development 6 (2002), 159-175.

Liebenberg, Sandra (2005): „The Value of Human Dignity in Interpreting Socio-Economic Rights«, in: South African Journal of Human Rights 21 (2005), 1-31.

Liebenberg, Sandra (2006): »Needs, Rights and Transformation: Adjudicating Social Rights«, in: Stellenbosch Law Review 17 (2006), 5-31.

Müller, Amrei (2009): »Limitations to and Derogations from Economic, Social and Cultural Rights«, in: Human Rights Law Review 9, 4 (2009), 557-601. 
Müller, Amrei (2013): The Relationship between Economic, Social and Cultural Rights and International Humanitarian Law, Leiden: BRILL.

Muralidhar, S. (2008): »India - The Expectations and Challenges of Judicial Enforcement of Social Rights«, in: Langford (2008), 102-124.

Nadakavukaren Schefer, Krista (Ed.) (2013): Poverty and the International Economic Legal System - Duties to the World's Poor, Cambridge: Cambridge University Press.

Nkongolo, Christian-Jr. Kabange (2014): »The Justiciability of Socio-Economic Rights under the African Charter on Human and Peoples' Rights: Appraisal and Perspectives Three Decades after its Adoption «, in: African Journal of International and Comparative Law 22 (2014), 492-511.

Nolan, Aoife (Ed.) (2014): Economic and Social Rights after the Global Financial Crisis, Cambridge: Cambridge University Press.

O’Cinneide, Colm (2014): »Austerity and the Faded Dream of a >Social Europe«, in: Nolan (2014), 169-201.

OHCHR (1986): »Limburg Principles on the Implementation of the ICESCR, June 1986«, Online: www.escr-net.org/docs/i/425445 [01.01. 2016].

OHCHR (2008): Report on Indicators for Promoting and Monitoring the Implementation of Human Rights, HRI/MC/2008/3, 6 June 2008.

Orago, Nicholas Wasonga (2015): »The Place of the >Minimum Core Approach in the Realisation of the Entrenched Socio-Economic Rights in the 2010 Kenyan Constitution«, in: Journal of African Law 59 (2015), 237-270.

Pieterse, Marius (2006): »Resuscitating Socio-Economic Rights: Constitutional Entitlements to Health Care Services«, in: South African Journal of Human Rights 22 (2006), 473-491.

Pillay, Karrisha (2002): »South Africa’s Commitment to Health Rights in the Spotlight: Do We Meet the International Standard?«, in: Brand/ Russell (2002), 61-70.

Pogge, Thomas W. (2002): World Poverty and Human Rights: Cosmopolitan Responsibilities and Reforms, Cambridge: Polity Press.

Politakis, George P. (Ed.) (2007): Protecting Labour Rights as Human Rights: Present and Future of International Supervision, International Colloquium on the 80th anniversary of the ILO Committee of Experts, Geneva, 24-25 November 2006, Geneva: ILO. 
Porter, Bruce (2005): »The Crisis of Economic, Social and Cultural Rights and Strategies for Addressing It «, in: Squires et al. (2005), 4-369.

Riedel, Eibe (2007): »Monitoring the 1966 International Covenant on Economic, Social and Cultural Rights«, in: Politakis (2007), 3-13.

Russell, Sage (2002): »Minimum State Obligations: International Dimensions«, in: Brand/Russell (2002), 11-22.

Scheinin, Martin (2001): »Economic and Social Rights as Legal Rights«, in: Eide et al. (2001), 111-122.

Schweizerisches Bundesgericht (1995): Einwohnergemeinde $X$ und Regierungsrat des Kantons Bern, BGE/ATF 121 I 367, 27. Oktober 1995.

Scott, Craig/Macklem, Patrick (1992): „Constitutional Ropes of Sand or Justiciable Guarantees? Social Rights in a New South African Constitution «, in: University of Pennsylvania Law Review 141, 1 (1992), 1-148.

Scott, Craig/Alston, Philip (2000): »Adjudicating Constitutional Priorities in a Transnational Context: A Comment on Soobramoney's Legacy and Grootboom's Promise«, in: South African Journal of Human Rights 16 (2000), 206-268.

Sen, Amartya (1984): »The Right Not to be Hungry«, in: Alston/ Tomaševski (1984), 69-82.

Sepulveda, Magdalena (2008): »Colombia: The Constitutional Court's Role in Addressing Social Injustice«, in: Langford (2008), 144-162.

South Africa: Constitutional Court (2002): Minister of Health and Others $v$ Treatment Action Campaign and Others (No 1), (CCT9/02) [2002] ZACC 16; 2002 (5) SA 703; 2002 (10) BCLR 1075 (5 July 2002).

Squires, John/Langford, Malcolm/Thiele, Bret (Eds.) (2005): The Road to a Remedy: Current Issues in the Litigation of Economic, Social and Cultural Rights, Sydney: UNSW Press.

Ssenyonjo, Manisuli (2009): Economic, Social and Cultural Rights in International Law, Oxford: Hart.

Supreme Court of India (1996): Paschim Banga Khet Majoor Samity v. State of West Bengal, 6 May 1996.

Van Bueren, Geraldine (1999): »Alleviating Poverty through the Constitutional Court«, in: South African Journal of Human Rights 15 (1999), 52-57.

Van Bueren, Geraldine (2002): „Of Floors and Ceilings: Minimum Core Obligations and Children«, in: Brand/Russell (2002), 183-200. 
Wesson, Murray (2004): „Grootboom and Beyond: Reassessing the Socio-Economic Jurisprudence of the South African Constitutional Court«, in: South African Journal of Human Rights 20 (2004), 284-308. White, Nigel (2007): »Applicability of Economic and Social Rights to the UN Security Council«, in: Baderin/McCorquodale (2007), 89-108.

World Health Organization (1978): Declaration of Alma-Ata, International Conference on Primary Health Care, Alma-Ata, USSR, 6-12 September 1978.

Young, Katharina (2008): »The Minimum Core of Economic and Social Rights: A Concept in Search of Content«, in: Yale Journal of International Law 32 (2008), 113-138. 


\title{
Universelle Gesundheitssicherung
}

Konzeptionelle Grundlagen und der

\author{
Beitrag Nationaler Ethikräte
}

\author{
CHRISTINA HEINICKE, LOTTA ERIKSSON,
} ABHA SAXENA, ANDREAS REIS

\section{EinLeitung ${ }^{1}$}

1948 wurde mit der Allgemeinen Erklärung der Menschenrechte auch das Recht auf Gesundheit proklamiert. ${ }^{2}$ Dieses Recht ist trotz seiner normativen Erhärtung im Internationalen Pakt über wirtschaftliche, soziale und kulturelle Rechte sowie auch praktische Fortschritte bisher allerdings noch keineswegs weltweit anerkannt und ist leider in einigen Regionen der Welt allenfalls bruchstückhaft realisiert. Die internationale Gemeinschaft hat verschiedene Versuche unternommen, dies zu ändern, zum Beispiel mit den Millennium Development Goals, die bis 2015 u.a. eine erhebliche Reduktion der Kindersterblichkeit weltweit verfolgten und auf diesem Gebiet auch beträchtliche Fortschritte nachweisen konnten. ${ }^{3}$ Ein Schwachpunkt

1 Vgl. auch den englischen Beitrag Heinicke et al. (2014), der für den vorliegenden Aufsatz grundlegend überarbeitet und aktualisiert wurde.

2 Vgl. United Nations (1948), 5 sowie United Nations (2000a). Heinicke et al. (2014).

3 Siehe dazu United Nations (2000b) und United Nations (2015), 5-6 bzw. 34-51. 
vieler dieser Ansätze und den daraus folgenden Gesundheitsprogrammen ist die Fokussierung auf einzelne Personengruppen oder Krankheitsbilder. Die Weltgesundheitsorganisation (WHO) präsentiert mit ihrem Konzept der universellen Gesundheitssicherung (engl. Universal Health Coverage/UHC) alternativ einen ganzheitlichen Ansatz zur Umsetzung des Rechts auf Gesundheit. Dieses Konzept soll in den ersten Abschnitten kurz erläutert sowie praktische Vorschläge und Hilfsmittel zur Sicherstellung von Gerechtigkeit auf dem Weg zu universeller Gesundheitssicherung sollen aufgezeigt werden.

Neben der WHO braucht es freilich auch nationale Instanzen, die als Fürsprecher für das Recht auf Gesundheit eintreten und eine gerechte Verteilung von Gesundheitsgütern national wie auch international einfordern. Diese Aufgabe kann und wird teilweise bereits von Nationalen Ethikräten wahrgenommen. In Zusammenarbeit mit der UNESCO und den Teilnehmenden des 10. Global Summits of National Ethics/Bioethics Committees 2014 in Mexico führte die Ethikabteilung der WHO erstmals eine empirische Umfrage zum Thema Nationale Ethikräte und Verteilungsgerechtigkeit im Gesundheitswesen durch. Die Ergebnisse der Umfrage sowie eine qualitative Analyse der Positionspapiere einzelner Ethikräte werden im dritten Teil des Artikels vorgestellt. Im Schlussteil sollen mit Beispielen aus den Niederlanden und Thailand konkrete Versuche zur Umsetzung bzw. zum Erreichen einer angemessenen Gesundheitsversorgung aufgezeigt werden.

\section{Das Konzept Universelle Gesundheitssicherung}

Die Weltgesundheitsorganisation (WHO) definiert universelle Gesundheitssicherung (UGS) (engl. Universal Health Coverage/UHC) als Sicherstellung dessen, dass allen Menschen die von ihnen benötigten Gesundheitsleistungen zur Verfügung stehen - seien sie präventiver, kurativer oder palliativer Art. Dabei müssen diese Leistungen von ausreichender Qualität sein, so dass sie auch wirksam sind, und gleichzeitig soll gewährleistet sein, dass die Inanspruchnahme dieser Leistungen die Betreffenden nicht in finanzielle Bedrängnis bringt. Die Definition betont demnach drei Aspekte: den Zugang zu Gesundheitsleistungen, die Qualität der angebotenen Leistungen und die Finanzierbarkeit seitens der Patienten. Um dieses Konzept 
zu verwirklichen, sind abgesehen vom Gesundheitssektor auch Verbesserungen in Bereichen wie Bildung, Verkehrswesen und Gesetzgebung erforderlich.

Häufig wird UGS als bloße Forderung nach einer allgemeinen Krankenversicherung verstanden. Dieses Denken greift allerdings zu kurz. Obwohl ein solides Finanzkonzept und die Abschaffung von direkten Zahlungen bei Leistungserhalt zweifellos eine wichtige Rolle spielen, sind diese Maßnahmen lediglich Teile eines ambitionierteren und weiter reichenden Ziels. UGS will letztlich sämtliche Hindernisse ausräumen, die Menschen davon abhalten, adäquate Gesundheitsleistungen zu erhalten; dies bezieht sich auf Hindernisse finanzieller, rechtlicher, organisatorischer, technischer, geographischer oder kultureller Art. Eine flächendeckende Gesundheitsversorgung allein genügt demnach zur vollständigen Umsetzung des Rechts auf Gesundheit nicht; es müssen auch die zugrunde liegenden weiteren Determinanten von Gesundheit - wie beispielsweise Bildungsstand, Umweltverschmutzungen und Arbeitssituation - erkannt und in Angriff genommen werden. Wer das Konzept der Universellen Gesundheitssicherung so in seiner Gänze begreift, wird zum Schluss kommen, dass derzeit kein Land eine Universelle Gesundheitssicherung erreicht hat. Vielmehr ist UGS als Ideal zu verstehen, auf das jedes Land schrittweise hinarbeiten soll.

Die erheblichen Unterschiede in der Gesundheitsversorgung innerhalb eines Landes, aber auch im Vergleich zwischen den Ländern, verlangen lokale wie auch internationale Maßnahmen. Ein entscheidender klärungsbedürftiger Punkt ist die Frage, inwieweit die internationale Gemeinschaft verpflichtet ist, finanzielle Unterstützung für diejenigen Staaten zu leisten, die lokale Bedürfnisse ihrer Bevölkerung in punkto Gesundheitssicherung nicht erfüllen können. Dabei gilt es zu bedenken, dass Universelle Gesundheitssicherung und das Recht auf Gesundheit nicht auf bestimmte staatliche Territorien beschränkt werden können.

\section{Ethische HERAUSFORDERUNGEN BEI DER UMSETZUNG VON UNIVERSELLER GESUNDHEITSSICHERUNG}

Bei der Umsetzung der Universellen Gesundheitssicherung müssen häufig schwierige ethische Entscheidungen getroffen werden. In einer Welt begrenzter Ressourcen, sowohl in Bezug auf Finanz- als auch auf Humanka- 
pital, sind Abwägungen zwischen verschiedenen Investitionen unvermeidlich. Gerade im Bereich des Gesundheitswesens haben solche Entscheidungen für einzelne Menschen allerdings womöglich weitreichende Folgen hinsichtlich ihrer Lebenserwartung und Lebensqualität. Es ist deswegen von größter Bedeutung, entsprechende Entscheidungen fair und verantwortlich zu treffen und sicherzustellen, dass die Resultate sowie die Entscheidungen selbst öffentlich bekannt gemacht werden und moralisch vertretbar sind.

Wer bekommt Zugang zu welchen Gesundheitsleistungen? Inwieweit muss das individuelle Leid dem Gemeinwohl untergeordnet werden? Wie kann ein Gesundheitssystem von Direktzahlungen zu Vorauszahlungen im Sinne einer Krankenversicherung übergehen? Inwiefern kann der Einzelne für seinen Gesundheitszustand zur Rechenschaft gezogen werden? Wenn Entscheidungsträger versuchen, solche Fragen zu lösen, könnten sie in Nationalen Ethikräten einen unterstützenden Partner finden. Diese können dabei helfen, die zugrunde liegenden ethischen Fragen zu identifizieren und Regierungen beraten, moralisch vertretbare Wege bei der Umsetzung von UGS einzuschlagen. Im Laufe der Zeit hat es zahlreiche Versuche gegeben, substanzielle Kriterien und faire Entscheidungsprozesse für Allokation im Gesundheitswesen zu erarbeiten. Eine Auswahl wird im Folgenden zusammenfassend vorgestellt.

\subsection{Der WHO-Report »Faire Entscheidungen auf dem Weg zu Universeller Gesundheitssicherung“}

2014 veröffentlichte die WHO Beratungsgruppe zu Gerechtigkeit und Universeller Gesundheitssicherung ihren Abschlussbericht »Making fair choices on the path to universal health coverage«. Die interdisziplinär zusammengesetzte Gruppe umfasste Mitglieder aus den Bereichen Ethik, Wirtschaft, Medizin und Rechtswissenschaft.

Der Bericht entwirft ein dreistufiges Vorgehen: In einem ersten Schritt sollen die Länder Gesundheitsleistungen nach Prioritätsgruppen kategorisieren. Dabei werden Kosteneffizienz, Schutz sozioökonomisch Schwacher und Schutz vor finanziellen Notlagen als Kriterien empfohlen. Innerhalb dieser Kriteriologie plädiert die Beratungsgruppe dafür, zunächst das Kriterium der Kosteneffektivität anzulegen und erst dann die anderen beiden 
Kriterien anzuwenden. ${ }^{4}$ Im zweiten Schritt sollen sodann sukzessive die hochpriorisierten Leistungen für die gesamte Gesellschaft verfügbar gemacht werden, bevor bestimmten Gruppen der Zugang zu weniger wichtigen Leistungen ermöglicht wird. Dabei sollen insbesondere finanzielle Hindernisse zum Zugang zu Gesundheitsleistungen angegangen werden (Abschaffung von Direktzahlungen, verpflichtendes Bündeln der Vorauszahlungen, einkommensabhängige Zahlungen). Der dritte Schritt besteht darin, vulnerable Gruppen der Gesellschaft (ländliche Bevölkerung, Bedürftige, Frauen und Kinder) besonders zu berücksichtigen.

Dem Beraterstab sind die finanziellen Grenzen mancher Länder durchaus bewusst, und man ist sich im Klaren darüber, dass zwischen verschiedenen Leistungen und den Entscheidungskriterien immer wieder abgewogen werden muss. Gleichzeitig identifiziert die Beratungsgruppe ex negativo fünf Entscheidungsmuster, die jedenfalls ethisch nicht akzeptabel wären:

- Inakzeptabel sind Entscheidungen, die Gesundheitssicherung auf weniger wichtigere Leistungen ausweiten, bevor die Gesamtbevölkerung auch nur annähernd Zugang zu den hochpriorisierten Leistungen hat.

- Inakzeptabel ist es, teuren, wenig effektiven Gesundheitsleistungen Vorrang zu geben, solange billigere und effektive Leistungen nicht flächendeckend übernommen werden.

- Inakzeptabel ist es, Leistungen zunächst für Menschen mit höherem sozioökonomischem Status zur Verfügung zu stellen, bevor vulnerable Gruppen abgedeckt sind (zumindest sofern sich die Kosten für die Versorgung nicht deutlich unterscheiden).

- Inakzeptabel ist es, zunächst diejenigen zu versorgen, die imstande sind, für Leistungen zu bezahlen.

- Inakzeptabel ist es, ein Vorschusssystem einzuführen, das die schrittweise Realisierung von UGS verlangsamt. ${ }^{5}$

Die verwendeten Kriterien und die Entscheidungen, wie diese abgewogen werden sollen, müssen Teil einer öffentlichen Debatte sein. Entscheidungsprozesse sollen institutionalisiert werden und definierten Qualitätsansprü-

\footnotetext{
4 Vgl. WHO (2014b), 11-24.

5 Vgl. WHO (2014b), S. 37-40.
} 
chen Rechnung tragen. Die WHO empfiehlt die Verwendung des »Accountability for Reasonableness«-Ansatzes, der in den folgenden Abschnitten kurz erläutert wird. Ein starkes Evaluations- und Kontrollsystem wird als ein essenzieller Bestandteil der Umsetzung von UGS gesehen. Dabei sollten Indikatoren den Fortschritt nicht nur aggregieren, sondern auch in Bezug auf vulnerable Gruppen darstellen. ${ }^{6}$

\subsection{Der Ansatz »Accountability for Reasonableness»}

Dieser Ansatz, der zuerst von Daniels und Sabin vorgestellt wurde, konzentriert sich auf die Fairness in Entscheidungsprozessen, also auf prozedurale Gerechtigkeit. Dies tun sie, weil eine Einigung in Bezug auf generelle, allgemeingütige Prinzipien zur Konfliktlösung bei Güterzuteilung als sehr schwierig bis unmöglich erachtet wird. ${ }^{7}$ Inwieweit ein gerechter Prozess mit Hilfe der Anwendung des »Accountability for Reasonableness «Ansatzes auch zu gerechteren Resultaten führt, wird debattiert. ${ }^{8}$

Folgt man dem Ansatz müssen vier Kernkriterien erfüllt sein, um einen gerechten und legitimen Entscheidungsprozess sicherzustellen:

- Die Kriterien für Allokationsentscheidungen müssen für die Öffentlichkeit zugänglich sein (Öffentlichkeitskriterium).

- Diese Kriterien müssen von fair denkenden Menschen - also denjenigen, die eine gemeinsame, allgemeingültige Rechtfertigung für ihre Entscheidungen im Sinn haben - für relevant gehalten werden (Relevanzkriterium).

- Es muss einen etablierten Weg geben, um Entscheidungen und die Kriterien, auf denen diese basieren, anzufechten (Anfechtbarkeitskriterium).

- Zusätzlich muss die Einhaltung der ersten drei Kriterien sichergestellt werden (Implementierungskriterium). ${ }^{9}$

6 Vgl. WHO (2014b), 46-47.

7 Vgl. Daniels/Sabin (2008) und Gruskin/Daniels (2008).

8 Vgl. Friedman (2008).

9 Vgl. Daniels/Sabin (2008). 
Der Ansatz erfreut sich international großer Beliebtheit und wurde in mehreren Studien auf seine praktische Anwendbarkeit hin getestet. ${ }^{10}$ "Accountability for Reasonableness « wird von Daniels selbst als Zwischenweg zwischen expliziter und impliziter Priorisierung gesehen, da es nicht darum geht, im Vorhinein explizite Kriterien zu benennen, wohl aber Transparenz und explizite Begründungen für die Resultate von Entscheidungsprozessen gefordert werden. ${ }^{11}$

\subsection{Multi-kriterielle Entscheidungsanalyse (MCDA)}

Wenn sich Entscheidungsträger schwierigen Fragen gegenüber positionieren sollen, bedienen sie sich oft eines intuitiven Zugangs, der schwierige Sachverhalte oft grob vereinfacht und wichtige Informationen außer Acht lässt. Um fair zwischen verschiedenen Kriterien abwägen zu können, müssen dabei neue Analysetechniken gefunden werden. In letzter Zeit wurde daher das Instrument der multi-kriteriellen Entscheidungsanalyse für den Gesundheitssektor adaptiert. ${ }^{12}$ Diese Form der Analyse findet bereits in anderen Bereichen Anwendung, wie zum Beispiel in den Umwelt- oder Agrarwissenschaften.

Um eine vergleichende Gesamtbeurteilung verschiedener Gesundheitsleistungen vornehmen zu können, müssen zunächst relevante, messbare Kriterien definiert werden (z.B. Kosteneffektivität, Schwere der Erkrankung oder Prävalenz der Erkrankung in vulnerablen Gruppen). Danach wird jede Leistung im Hinblick auf jedes dieser Kriterien hin evaluiert. Dabei ist es auch möglich, die Kriterien unterschiedlich zu gewichten, um noch klarere Ergebnisse zu erzielen. Zusätzlich gilt es zu entscheiden, ob eine Güterabwägung akzeptiert werden kann, das heißt, ob gute Resultate in einer Kategorie schlechte Ergebnisse in einer anderen Kategorie ausgleichen können. Das Resultat ist eine Rangliste der geprüften Leistungen, die dann als objektive Entscheidungsgrundlage dienen soll.

10 Siehe dazu z.B. Kapiriri et al. (2009), Maluka et al. (2011) und Martin et al. (2002).

11 Vgl. Daniels (2000).

12 Vgl. Peacock et al. (2009); Youngkong et al. (2012). 


\section{Beiträge Nationaler ETHIKRÄTE ZUR UNIVERSELLEN GRUNDSICHERUNG}

Beim Versuch, eine gerechte Verteilung von Ressourcen im Gesundheitswesen zu erreichen, können Regierungen auf Nationale Ethikräte als Beratungsinstanzen zurückgreifen. Dies geschieht vor allem in Westeuropa mit großer Regelmäßigkeit, die hier ansässigen Ethikräte sind gut etabliert und als Partner von Entscheidungsträgern anerkannt. In den folgenden Abschnitten findet sich eine Zusammenstellung der Stellungnahmen Nationaler Ethikräte zu den Themen Kosteneffektivität, fairer Ressourcenallokation und globaler Gerechtigkeit im Gesundheitswesen, die auf einer OnlineRecherche aller offiziellen Internetauftritte Nationaler Ethikräte basiert.

\subsection{Kosteneffektivität}

Obgleich in vielen Ländern der Kosteneffektivität ein hoher Stellenwert in Entscheidungsprozessen zugestanden wird, haben Nationale Ethikräte in der Vergangenheit mehrere kritische Stellungnahmen zu diesem Kriterium veröffentlicht. Die Anwendung von »QALYs« (Bewertung eines Lebensjahrs in Relation zur Gesundheit) pro Kosteneinheit im Vergleich verschiedener Patientengruppen und Indikationen (z.B. der Vergleich einer Blinddarmentfernung mit der Behandlung von Diabeteskranken) wird als besonders kritisch bewertet. Stellungnahmen der Ethikräte aus Deutschland, ${ }^{13}$ Frankreich, ${ }^{14}$ und den Niederlanden ${ }^{15}$ machen auf Unzulänglichkeiten des QALY-Systems aufmerksam: fehlende Gewichtung der Schwere einer Erkrankung, systematische Diskriminierung gegenüber Menschen mit chronischer Krankheit oder Behinderung, Benachteiligung von Patienten mit unheilbaren Krankheitszuständen und Unterschätzung von kurzen, aber extrem pathologischen Zuständen. Obwohl die vorgebrachten Bedenken durch Modifikation des QALY-Systems möglicherweise zerstreut werden könnten (z.B. durch eine Art Gerechtigkeitsgewichtung), halten die Ethikräte

13 Vgl. Deutscher Ethikrat (2011).

14 Vgl. CCNE (1998).

15 Vgl. CEG (2005). 
dies einstimmig für wenig vielversprechend. ${ }^{16}$ In ihren konkreten Schlussfolgerungen unterscheiden sich die Stellungnahmen im Übrigen dann aber deutlich. Während das deutsche Papier eine Gesetzesänderung fordert, welche explizit den Einsatz von QALYs als Grundlage für Kosten-NutzenAnalysen über Indikationsgrenzen hinweg verbieten soll, fordern das niederländische und das französische Pendant lediglich, sich der Grenzen des QALY-Systems bewusst zu sein und darauf basierende Kosten-NutzenAnalyse nur als nur eines von verschiedenen Werkzeugen zur Evaluation von Gesundheitsleistungen zu sehen.

Weiterhin betonen alle Veröffentlichungen, dass eine Ressourcenverschwendung zutiefst unmoralisch sei. Wo Menschen von einem effektiveren Einsatz von Mitteln und einer effizienteren Behandlung profitieren, stimmten Kosteneffektivitätsanalysen und ethische Empfehlungen voll überein.

\subsection{Auf dem Weg zu fairen Entscheidungen}

Die veröffentlichten Stellungnahmen Nationaler Ethikräte sind sich einig darin, dass wenn Rationierung und Priorisierung im Gesundheitswesen stattfinden, diese explizit und öffentlich vonstattengehen sollten. Leider wird dies in der Realität nicht durchgehend praktiziert.

Ein Modell des portugiesischen Ethikrats ${ }^{17}$ zur Rückerstattung medizinischer Produkte basiert größtenteils auf dem »Accountability for Reasonableness«-Ansatz, adaptiert um das Kontinuitätsprinzip (d.h. aktuelle Entscheidungen sollten stringent zusammenhängen mit vorangegangenen) und einem Effektivitätskriterium (d.h. Entscheidungen sollten zeitnah getroffen werden können). Es wird hier ein dreiteiliges Verfahren vorgeschlagen, beginnend mit einer klinischen Evaluationsphase, geleitet von Fachleuten aus medizinischer Praxis, Wissenschaft und Pharmazie, um die Angemessenheit bestehender Behandlungsmöglichkeiten für eine spezifische Indikation zu überprüfen. Der zweite Schritt soll eine Kosteneffektivitätsanalyse beinhalten unter Beteiligung von Krankenhausleitungen und

16 Vgl. Deutscher Ethikrat (2011), CCNE (1998), CEG (2005) und CNECV (2012).

17 Vgl. CNECV (2012). 
Patientenvereinigungen jeweils für die Behandlung eines spezifischen Krankheitsbildes. Der dritte und letzte Schritt ist die öffentliche Debatte.

Das französische Statement ${ }^{18}$ verbleibt bei weniger konkreteren Forderungen hinsichtlich der Implementierung von öffentlicher Beteiligung am Entscheidungsprozess. Darüber hinaus werden für andere Bereiche zwei Maßnahmen benannt, die vorrangig umgesetzt werden sollten: die systematische Einbindung von Patientenvereinigungen in Entscheidungsprozesse sowie eine verbesserte Ausbildung medizinischen Personals hinsichtlich ethisch vertretbarer Entscheidungsfindung. Das übergeordnete Ziel bleibt deutlich: Jedwede implizite Selektion oder Implementierung von Entscheidungskriterien, die nicht zuvor einer öffentlichen Debatte unterzogen worden sind, müssen abgelehnt werden.

Der dänische Ethikrat ${ }^{19}$ betont ebenfalls die Notwendigkeit, Entscheidungsträger gut auf Entscheidungssituationen vorzubereiten; dabei sind sowohl medizinisches Personal wie auch lokale und nationale politische Entscheidungsträger im Blick. Diese Gruppen sollen mit wissenschaftlichen Informationen zu möglichen Konsequenzen ihrer Handlungen vertraut gemacht werden. Außerdem muss deutlich erkennbar sein, welche Kompetenzen auf welcher Entscheidungsebene angesiedelt sind, um die Mitwirkung der Öffentlichkeit zu erleichtern und Möglichkeiten zur Anfechtung von Entscheidungen zu schaffen.

Das deutsche Positionspapier ${ }^{20}$ stellt klare Minimalforderungen an den Entscheidungsprozess: Entscheidungsträger müssen demokratisch legitimiert sein, Entscheidungen dürfen dementsprechend nicht an wissenschaftliche Institute oder Interessensgruppen delegiert werden, und das fundamentale Recht des Individuums auf Zugang zu angemessenen Gesundheitsleistungen darf nicht mit dem Ziel unterminiert werden, das kollektive, aggregierte Maß an Gesundheit einer Gesamtgesellschaft zu steigern.

18 Vgl. CCNE (1998).

19 Vgl. Det Etiske Råd (1996).

20 Vgl. Deutscher Ethikrat (2011). 


\subsection{Unterschiede in der Gesundheitsversorgung im globalen Kontext}

Das Thema der globalen Ungleichheiten in Bezug auf Gesundheit wurde bisher von Nationalen Ethikräten nur wenig bearbeitet. Lediglich der französische Ethikrat veröffentlichte 2003 eine Stellungnahme dazu. ${ }^{21}$ In diesem Positionspapier drängt der Rat die entwickelten Länder dazu, die Bedürfnisse und Wünsche von Entwicklungs- und Schwellenländern ernst zu nehmen. Die Bedürfnisse von Bevölkerungen müssen lokal eruiert werden und sollten nicht nur im Lichte »westlicher « Konzepte erhoben werden. Wo Meinungen voneinander abweichen, sollte die Entscheidung bei den Regierungen von Entwicklungsländern liegen, solange diese die Grundprinzipien der Menschenwürde achten. Finanzielle und technische Unterstützung von Entwicklungsländern ist in den Augen des Rates keine Frage der Großzügigkeit, sondern wird vielmehr zur Verpflichtung, sobald Regierungen das Recht auf Gesundheit als universelles Recht anerkennen. Weiterhin wird darauf hingewiesen, dass sich ein Konflikt zwischen Patenthaltern medizinischer Produkte und Gesundheitsaktivisten zuspitzt. Hier sollen internationale Organisationen wie die Welthandelsorganisation und die Weltgesundheitsorganisation, vermittelnd eingreifen und zusammenarbeiten. Außerdem warnt die Stellungnahme vor sogenannten vertikalen Hilfsprogrammen, d.h. Programmen, die sich ausschließlich um Patientinnen und Patienten mit einer bestimmten Erkrankung kümmern, wie beispielsweise viele HIV/AIDS- oder Tuberkulose-Programme. Diese könnten einen ganzheitlichen, patientenzentrierten Ansatz im Gesundheitswesen leicht unterminieren.

\subsection{Analyse einer Online-Umfrage unter Nationalen Ethikräten zum Thema Priorisierung}

In Vorbereitung auf das Gipfeltreffen Nationaler Ethikräte (Global Summit of National Ethics/Bioethics Committees) 2014 in Mexiko wurde vom Sekretariat des Global Summits (Ethics Unit der WHO) in Zusammenarbeit mit der UNESCO ein Online-Fragebogen an 109 Personen oder Institutionen verschickt. Neben Fragen zur allgemeinen Konstitution und Arbeitsweise

21 Vgl. CCNE (2003). 
der einzelnen Ethikräte wurden auch Fragen nach existierenden Priorisierungsmechanismen und Universeller Gesundheitssicherung gestellt. Der Fragebogen war auf Englisch, Französisch, Spanisch und Portugiesisch verfügbar. Die Mehrheit der Antworten kam aus dem europäischen und afrikanischen Bereich (32 von 42 Antworten). Die Rücklaufquote betrug 38,5 Prozent.

91 Prozent der Befragten berichteten, dass es eine strukturierte Vorgehensweise für Priorisierungen im Gesundheitswesen in ihrem Land gäbe. Die meisten dieser Verfahrensweisen sind auf nationaler Ebene etabliert (84\%) und werden durch entsprechende Gesetzgebung garantiert oder von spezialisierten Regierungsorganisationen verantwortet. Diese Verfahren beruhen laut Umfrage vorwiegend auf den Prinzipien von Menschenwürde (63,6\% Zustimmung), Gerechtigkeit (63,6\%) und Kosteneffektivität (56,8\%). Weniger berücksichtigt würden Prinzipien wie die Verantwortung des Einzelnen (29,6\%) oder die öffentliche Akzeptanz (34\%).

38,2 Prozent der Befragten gaben an, dass kein explizit ethischer Rat für Priorisierungsentscheidungen eingeholt wird oder wurde. Diese Lücke könnten Nationale Ethikräte schließen und die Beachtung ethischer Prinzipien in der Entscheidungsfindung weiter vorantreiben. Dass sie diese Rolle übernehmen könnten, zeigt sich in den Ländern, in denen aktiv ethischer Rat von Entscheidungsträgern gesucht worden ist - in 90,4 Prozent der Fälle wandte man sich an den Nationalen Ethikrat.

Das Konzept der Universellen Gesundheitssicherung als solches und die Allokation von Finanz- und Humankapital im Gesundheitswesen wurde bisher noch nicht differenzierter in Ethikräten diskutiert. Größtenteils sind es die Ethikräte Europas, die Stellungnahmen veröffentlicht, Workshops abgehalten, Konferenzen ausgerichtet oder Öffentlichkeitskampagnen gestartet haben. Eine Erklärung könnten die unterschiedlichen Kompetenzen und Aufgabenbereiche Nationaler Ethikräte in ihren jeweiligen Heimatländern liefern, sowie ihr unterschiedlich langes Bestehen. Diesen Schluss legen zumindest die Antworten des Fragebogens nahe: 42,9 Prozent der antwortenden Ethikräte berichteten, kein Mandat zu haben, um zu Fragen der öffentlichen Gesundheitspflege Stellung zu nehmen, oder der betroffene Ethikrat war erst kurz zuvor etabliert worden.

In einer Welt begrenzter Ressourcen sehen sich Länder auf dem Weg zu einer Universellen Gesundheitssicherung mit schwierigen Fragen konfrontiert. Um zu verhindern, dass Entscheidungen allein auf Kosteneffektivi- 
tätskriterien basieren, und um zu erreichen, dass andere ethische Prinzipien wie Gerechtigkeit und Berücksichtigung vulnerabler Gruppen Eingang in Entscheidungsprozesse erhalten, braucht es starke Fürsprecher und solide Rahmenkonzepte. Dies ist insbesondere auch für die Länder relevant, in denen künftig aus Kostengründen bestimmte Gesundheitsleistungen nicht mehr von Krankenversicherungen übernommen werden sollen. Hier müssen Regierungen starke und moralisch vertretbare Argumente vorbringen, um die Öffentlichkeit von der Legitimität und Notwendigkeit ihrer Handlungen zu überzeugen.

Nationale Ethikräte können zur schrittweisen Umsetzung Universeller Gesundheitssicherung in vielfacher Weise beitragen. Zunächst können sie die Öffentlichkeit informieren und über die grundlegende Problematik der Allokation im Gesundheitswesen aufklären. Zweitens können sie direkt zur Entwicklung oder Adaptation von ethischen Rahmenkonzepten beitragen. Drittens können sie für Transparenz im Entscheidungsprozess eintreten. Zu guter Letzt können sie die Gesellschaft und die Regierungen immer wieder mahnend daran erinnern, dass es sich bei dem Recht auf Gesundheit um ein globales Menschenrecht handelt und so zu einem Voranschreiten hin zu einer tatsächlich universellen Gesundheitssicherung beitragen.

\section{Der Kampf um eine angemessene GESUNDHEITSVERSORGUNG: BEISPIELE AUS DEN NIEDERLANDEN UND THAILAND}

In den folgenden Kapiteln geht es darum, die Leserschaft mit dem Reformprozess im Gesundheitswesen zweier Länder näher vertraut zu machen. Thailand steht dabei für die aufstrebenden Entwicklungs- und Schwellenländer, die durch neuen Kapitalfluss und Umverteilung von Ressourcen in die Lage versetzt werden, zusätzliche Gesundheitsleistungen in staatliche Programme aufzunehmen. Im Gegensatz dazu wird am Beispiel der Niederlande illustriert, wie Industrienationen steigende Kosten im Gesundheitssektor einzudämmen versuchen, ohne dabei sozialer Ungerechtigkeit Vorschub zu leisten. Die Entwicklung teurer neuer Medikamente und Technologien sowie eine alternde Gesamtgesellschaft vergrößern auch in den Wohlfahrtsstaaten des Westens die Lücke zwischen dem medizinisch Möglichen und dem (staatlich) Finanzierbaren. 


\subsection{Die Niederlande}

\section{Hintergrund}

Die niederländische Regierung erkannte als eine der ersten in Europa die wachsende Bedeutung von Priorisierung und Rationierung im Gesundheitssektor aufgrund steigender Kosten. Das sogenannte »Dunning Komitee« wurde bereits 1990 berufen, um sich über mögliche Kriterien im Prozess der Priorisierung Gedanken zu machen. Die Ergebnisse des Komitees wurden 1991 der Regierung präsentiert und umfassten vier Kriterien für die Evaluierung von Gesundheitsleistungen: Notwendigkeit, Effektivität (d.h. die Maßnahme muss in der Lage sein, ein Ziel zu erreichen), Effizienz (ausgerichtet an einer Kosten-Nutzen-Bilanzierung) und Eigenverantwortung (orientiert an zumutbaren Kosten für den Einzelnen). ${ }^{22}$ Obwohl es sich als schwierig herausstellte, diese Kriterien direkt in die Praxis umzusetzen, bilden sie auch heute noch das Rückgrat für Entscheidungen über Aufnahme oder Streichung von Leistungen aus dem Nationalen Gesundheitspaket.

Das Gesundheitssystem der Niederlande hat sich innerhalb der letzten Jahrzehnte drastisch verändert. Ein komplexes System, getragen durch gesetzliche und private Krankenversicherungsgesellschaften, das auf drei großen staatlichen Programmen beruhte, wurde 2006 aufgelöst. Seitdem gibt es eine gesetzlich vorgeschriebene Pflicht, sich privat zu versichern. Jeder Bewohner muss eine Langzeitpflegeversicherung abschließen und sich für eine festgelegte Anzahl von Leistungen versichern lassen. Dieser Pflichtleistungskatalog umfasst zahlreiche Dienste und schließt nur wenige medizinische Leistungen aus wie Schönheitschirurgie, aber auch Zahnarztkosten für Erwachsene. Zur Finanzierung zahlt jeder Steuerzahler 12,5 Prozent seines Einkommens in die Langzeitpflege und kauft zusätzlich die Versicherung für die anderen Pflichtleistungen. Die Versicherungsgesellschaften sind dazu verpflichtet, die Basisversicherung jeder Person mit gültigem Aufenthaltsstatus zum selben Preis anzubieten, unabhängig von dessen Einkommen oder dessen Vorerkrankungen. Der Staat verteilt Subventionen, um ein Ungleichgewicht von Patienten mit hohen Gesundheitskosten zwischen den Versicherungsfirmen auszugleichen. Zusätzlich bieten die Versicherer ein breites Spektrum von Zusatzversicherungen an. Den Versi-

22 Vgl. Hermans/den Exter (1998). 
cherern steht es frei, ob sie gewinnorientiert oder als Non-Profit-Organisation arbeiten wollen. ${ }^{23}$

Für den Pflichtleistungskatalog bezahlen die Niederländer auf verschiedene Art und Weise: Jeder Versicherte bezahlt eine Prämie direkt an seinen Versicherer. Diese Kosten variieren je nach Firma. Zusätzlich behalten die Arbeitgeber einen Prozentsatz des Gehalts zur Finanzierung der Versicherung ein (Selbstständige zahlen einen festgesetzten Prozentsatz ihres einkommenssteuerpflichtigen Ertrags). Überdies stellt der Staat Zuschüsse für Kinder unter 18 Jahren und einkommensschwache Haushalte sowie Fördermittel für Forschung und Interessen der öffentlichen Gesundheit (Impfprogramme, Hygiene, Datenerhebung) bereit. Jeder Versicherte zahlt einen Eigenanteil von 165 Euro pro Jahr, bevor die Versicherung zahlungspflichtig wird. ${ }^{24}$

Die Idee hinter diesen Reformen war es, die Prinzipien eines regulierten Marktes im Gesundheitssektor einzuführen, mit dem Ziel, durch den entstehenden Konkurrenzdruck kostensparende, effiziente Lösungen für die Versicherung der Gesamtgesellschaft zu erhalten. Versicherern steht es frei, mit Einzelpersonen Verträge abzuschließen oder aber Arbeitgeber direkt anzusprechen und ihnen Gruppentarife für ihre Beschäftigten anzubieten. Zusätzlich steht es den Versicherern frei, Programme anzubieten, die im Austausch für niedrigere monatliche Beiträge entweder einen höheren Selbstzahlerbeitrag ansetzen (bis zu $500 €$ Eigenbeteiligung/Jahr) oder den Zugang zu Gesundheitsleistungen auf feste Partnerinstitutionen beschränken. Ungefähr 90 Prozent der Niederländer schließen Zusatzversicherungen $\mathrm{ab}^{25}$

Die Primärversorgung durch Hausärzte stellt auch nach den Reformen einen wichtigen Baustein im Gesundheitssystem dar. Ohne Ein- oder Überweisung wird eine Behandlung im Sekundärbereich nur in Notfällen übernommen.

\section{Der Entscheidungsprozess}

In der Gesetzgebung zur verpflichtenden privaten Krankenversicherung findet sich lediglich eine funktionelle Beschreibung des Pflichtleistungska-

23 Schäfer et al. (2010).

24 Ebd.

25 Vgl. den Exter (2010). 
talogs. Die Aufnahme einzelner Leistungen in den Katalog wird letztendlich vom Gesundheitsministerium beschlossen, das diesbezüglich vom Gesundheitsversicherungsausschuss beraten wird. Dieser Ausschuss klärt auch Fragen zum Umfang der zu erbringenden Leistungen und führt die Aufsicht über die staatlichen Fördertöpfe zur Subventionierung von Patientengruppen mit hohen Gesundheitskosten. Der Gesundheitsversicherungsausschuss ist eine unabhängige Organisation, deren Aufsichtsratsmitglieder vom Gesundheitsminister ernannt werden. Die Evaluierung von Gesundheitsleistungen basiert auf den bereits erwähnten Dunning-Kriterien: Notwendigkeit, Effektivität, Effizienz und Eigenverantwortung. Um beim Priorisieren sowohl Kosteneffektivität wie auch Gerechtigkeitsüberlegungen zu berücksichtigen, benutzt der Ausschuss als Maß gewichtete Kosten per QALY (Verlust an qualitätskorrigierten Lebensjahren aufgrund der Erkrankung im Verhältnis zu verbleibender Lebenserwartung). Diese Gewichtung wird "proportional shortfall« (»verhältnismäßiges Defizit«) ${ }^{26}$ genannt und versucht das »fair innings « Kriterium mit Lebenserwartungsschätzungen und dem Prinzip des Vorrangs der Schlechtergestellten (priority to the worse off) in Einklang zu bringen. Bisher gibt es keine maximale Obergrenze für die Kosten pro gewichtetem QALY, obwohl die Zahl 80.000 €/QALY diskutiert wurde.

\section{Fairness und Gerechtigkeit nach der Einführung der verpflichtenden privaten Krankenversicherung}

Grundsätzlich ist es noch zu früh, um abschließend zu beurteilen, ob die Reformen zu den gewünschten Kostenersparnissen im Gesundheitssektor geführt haben, ohne eine gerechte Gesundheitsversorgung wesentlich zu behindern. Die entstandene Möglichkeit für Versicherungsgesellschaften, direkt mit Anbietern von Gesundheitsleistungen zu verhandeln, führte zu einigen ethischen Bedenken, vor allem im Hinblick auf die Beschränkung der freien (Arzt-)Wahl des Versicherten. Obwohl die Reformen dem einzelnen Bürger eine stärkere Verhandlungsposition und mehr Möglichkeiten zum Erwerb günstiger und guter Gesundheitsversorgung zusprechen wollten, nutzen in der Realität die wenigsten Niederländer diese Optionen. ${ }^{27}$ Diejenigen, die ihre Versicherung wechseln, sind im Großteil junge, ge-

26 Vgl. van de Wetering et al. (2013).

27 Vgl. Okma/Crivelli (2013). 
sunde, gebildete und gut verdienende Versicherte. Diese Tatsache wirft die Frage auf, ob Mitglieder vulnerabler Gruppen - wie zum Beispiel Senioren, sozial Schwache, Menschen mit Behinderung - in ausreichendem Maße die Möglichkeit haben, diese Optionen tatsächlich wahrzunehmen.

\section{Der Beitrag des niederländischen Zentrums für Ethik und Gesundheit}

Das Zentrum für Ethik und Gesundheit wurde vom Ministerium für Gesundheit, Gemeinwohl und Sport 2001 gegründet. Eine der Hauptaufgaben besteht darin, einen jährlichen Bericht über die Volksgesundheit aus ethischer Perspektive vorzulegen, der dann die Agenda des niederländischen Parlaments in Bezug auf Ethik und Gesundheit prägt. Das Zentrum hat wiederholt auf die Schwierigkeiten der Kosten-Nutzen-Analyse hingewiesen $^{28}$ und Stellung bezogen zu Themen wie dem Gebrauch wissenschaftlicher Beweise in der Gesundheitspolitik, ${ }^{29}$ dem Einfluss der Ökonomisierung des Gesundheitssystems auf das Berufsethos des Gesundheitspersonals ${ }^{30}$ sowie die Herausforderungen, denen sich Pflegepersonal und pflegende Angehörige durch neue Finanzierungsstrukturen gegenübersehen (2009). ${ }^{31}$

\subsection{Thailand}

\section{Hintergründe}

Vor der Einführung eines staatlichen Programms zur Universellen Gesundheitssicherung im Jahr 2001 waren circa 30 Prozent der thailändischen Bevölkerung nicht versichert, trotz zahlreicher Programme, die auf eine Versorgung der Beschäftigten im informellen Sektor und die armen Teile der Bevölkerung zielten. 2001 erholte sich Asien noch von der großen Finanzkrise 1997, und externe Berater zweifelten an der Umsetzbarkeit eines größer angelegten Gesundheitsprogramms. Trotzdem entschloss sich der damalige Regierungspräsident Thaksin Shinawatra, eine Universelle Gesundheitssicherung in sechs Regionen des Landes als Pilotversuch einzuführen;

\footnotetext{
28 Vgl. CEG (2005).

29 Vgl. CEG (2007a).

30 Vgl. CEG (2007b).

31 Vgl. CEG (2009).
} 
bereits 2002 wurde das Programm auf das ganze Land ausgedehnt. Das Programm wird durch allgemeine Steuereinnahmen finanziert, die einkommensabhängig berechnet werden, und garantiert kostenlosen Zugang für jeden außerhalb des formellen Sektors des Arbeitsmarktes. Die übrigen Teile der Gesellschaft werden über Programme für staatliche Angestellte oder die Krankenversicherung der Sozialversicherungspflichtigen abgedeckt. $^{32}$

Die rasche Ausdehnung der Gesundheitssicherung wurde durch erhebliche Investitionen in regionale Krankenhäuser und Gesundheitszentren in der Vergangenheit ermöglicht sowie durch die kontinuierliche Unterstützung durch die Zivilgesellschaft und selbst durch politische Gegner. ${ }^{33}$ Kostenloser Zugang wird für eine große Reihe an Leistungen gewährt. Tatsächlich finden sich nur wenige explizite Ausnahmen wie Fruchtbarkeitsbehandlungen oder Schönheitsoperationen. Die Wächterfunktion der Hausärzte spielt eine herausragende Rolle bei dem Versuch, die Kosten für die universelle Gesundheitssicherung in einem angemessenen Rahmen zu halten. Patienten im UGS-Programm müssen sich bei einem spezifischen Hausarzt registrieren und dürfen - außer in Notfällen - keine sekundären Zentren ohne Überweisungsschein aufsuchen. Der einzelne Hausarzt bezieht eine Pro-Kopf-Pauschale; Krankenhäuser erhalten ein Budget nach DRGs (Diagnosis Related Groups) aus einem gedeckelten Topf für sekundäre Gesundheitsversorgung.

\section{Der Entscheidungsprozess}

Thailand bedient sich eines Käufer-Anbieter-Systems, d.h. der Staat, repräsentiert durch eine unabhängige Organisation (National Health Security Office), kauft von Krankenhäusern spezielle Dienstleistungen oder handelt mit Pharmafirmen Preise für Medikamente aus. Die Organisation besitzt einen interdisziplinären Vorstand, dessen Vorsitzender der Gesundheitsminister ist. Im Vorstand sind die Zivilgesellschaft, Gesundheitsberufe und Klinikleitungen repräsentiert. Zu Beginn fielen Entscheidungen noch relativ ungeregelt, spontan oder intransparent und waren deswegen anfällig für Lobbyismus einflussreicher Interessensgruppen. Um diesen Problemen zu begegnen, etablierte das National Health Security Office 2009 eine Koope-

32 Vgl. Evans et al. (2012).

33 Vgl. Tangcharoensathien et al. (2013). 
ration mit einem unabhängigen wissenschaftlichen Institut, mit dessen Hilfe sie einen optimalen Entscheidungsprozess zu erlangen suchten. ${ }^{34}$ Die Ergebnisse führten zu einem vierstufigen Vorgehen:

- Gesundheitsleistungen werden von einem breiten Spektrum von Interessensgruppen zur Aufnahme in die universelle Gesundheitssicherung vorgeschlagen: Politiker, Ärzte, Patientengruppen, NGOs, Vertreter von Pharmazie und Medizinindustrie, Akademikern und Laien.

- Auswahl von Gesundheitsleistungen zur Evaluation von einem ähnlichen Spektrum von Interessengruppen unter Ausschluss von Industrie und Politikern, da hier ein zu großer Interessenkonflikt erwartet wird.

- Evaluation der Gesundheitsleistungen unter fünf Gesichtspunkten, die jeweils als gleich bedeutend gewertet werden: Prävalenz, Effektivität der Gesundheitsleistung (Vorzug von kurativen gegenüber rehabilitativen Interventionen, hohe Sensitivität und Spezifität von diagnostischen Tests etc.), Einfluss auf finanzielle Belastung des Haushalts der Patienten, bestehende Übernahme der Kosten für Gesundheitsleistungen in den anderen Versicherungsprogrammen, ethische und soziale Implikationen (bevorzugt Interventionen, die sozial Schwache erreichen, Berücksichtigung von Patienten mit seltenen Erkrankungen). Zusätzlich wurde für jede Leistung eine technologische Evaluation mit den Kriterien Kosten pro QALY und Einfluss auf das Gesamtgesundheitsbudget durchgeführt.

- Abschließende Bewertung durch einen Unterausschuss (Mitglieder aus Politik, Gesundheitspersonal, Zivilgesellschaft und Patientenorganisationen). Zusätzlich zu den Ergebnissen der durchgeführten Analyse wurde bewertet, ob bereits alternative, günstige Behandlungsmöglichkeiten für dieselbe Indikation im Gesundheitspaket enthalten waren und ob die Übernahme der Kosten finanzierbar für das Gesamtsystem ist (Grenzbetrag: einfaches Bruttoinlandsprodukt pro Kopf). ${ }^{35}$

34 Vgl. Youngkong et al. (2012).

35 Ebd. 


\section{Fairness und Gerechtigkeit nach der Einführung der Universellen Gesundheitssicherung}

Mit der Einführung eines Universellen Gesundheitssicherungskonzepts wurden auch Versuche unternommen, die Leistungen des Gesundheitssystems sowie basale Gesundheitsdaten der Bevölkerung häufiger und flächendeckender zu erheben. Die unterschiedliche Qualität und Menge der Gesundheitsdaten vor und nach der Einführung von UGS erschweren die Interpretation der Ergebnisse in einem gewissen Maß.

Ein umfassender Bericht über die Ergebnisse von UGS in Thailand wurde $2012^{36}$ veröffentlicht und enthält eine Analyse der Daten aus den ersten zehn Jahren des Programms. Darin konnte gezeigt werden, dass Gesundheitsleistungen häufiger in Anspruch genommen werden (ambulante Termine pro Mitglied im Programm stiegen von 2,45 im Jahr 2003 auf 3,22 in 2010) und dass der ungedeckte medizinische Bedarf in der Gesellschaft generell niedrig ist (für 1,44\% der ambulanten und 0,4\% der stationären Patienten bestand 2010 ungedeckter medizinischer Bedarf). Zur Armut führende Gesundheitskosten (definiert als Ausgaben von $>10 \%$ des Haushaltseinkommens) nahmen vor allem in den ärmsten Gesellschaftsschichten bedeutend ab (6,8\% in 1996 zu 2,8\% in 2008). Heutzutage sind es im Gegenteil eher die reichsten Teile der Bevölkerung, die Gefahr laufen, 10 Prozent ihres Einkommens in Gesundheitsleistungen zu investieren (4,7\% in 2009); dabei wird das Geld häufig an private Dienstleister bezahlt, um Wartezeiten im staatlichen Gesundheitssystem zu umgehen, oder es wird für Dienste ausgegeben, die noch nicht in das staatliche Programm aufgenommen wurden. $^{37}$

\section{Jetzige und künftige Herausforderungen}

Trotz beeindruckender Fortschritte steht Thailand noch vor einigen verbleibenden Problemen, und neue Herausforderungen sind bereits absehbar. Eine Studie, die Daten aus dem Jahr 2006 analysierte, zeigte beispielsweise, dass obwohl die Versorgung von Kindern und Müttern insgesamt zufriedenstellend war, erhebliche Unterschiede zwischen Reichen und Armen bestehen blieben. ${ }^{38}$ Insbesondere wurde dies deutlich bei Zahlen zu Tee-

36 Vgl. Evans et al. (2012).

37 Vgl. Somkotra/Lagrada (2009).

38 Vgl. Limwattananon et al. (2010). 
nagerschwangerschaften oder Fehl- und Mangelernährung von Kindern, die sehr eng mit dem Bildungsstand der Mütter verknüpft waren. Ähnliches zeigte auch eine Analyse der OECD aus 2010; ${ }^{39}$ hier wurde der ungedeckte medizinische Bedarf in einzelnen Bevölkerungsgruppen analysiert, Frauen, Arme und die Landbevölkerung wiesen auch hier den klar höchsten ungedeckten Bedarf auf. Zusätzlich bleiben einige ethische Probleme bisher ungelöst, unter anderem die Ungleichheit zwischen dem staatlichen Versicherungssystem der universellen Gesundheitssicherung und dem Versicherungsprogramm für Beamte sowie Probleme mit Wartezeiten auf Behandlungen (fehlende Daten zum Einsatz und Länge von Wartelisten), Fragen der Patientenautonomie bei eingeschränkter Arztwahl (durch das Überweisungssystem) und die Anforderungen an ein Gesundheitssystem bei einer zunehmend alternden Gesellschaft. ${ }^{40}$

Die Regierung von Thailand hat den Wert von expliziten Priorisierungsmechanismen und das transparente Abwägen ethischer Überlegungen erkannt und gefördert. Angesichts nach wie vor bestehender und künftig zu erwartender ethischer Konflikte würde Thailand von einem starken Nationalen Ethikkomitee gewiss profitieren. Ausgestattet mit dem Mandat, auch zu Fragen der Gesundheitspolitik Stellung zu nehmen, könnte es eine Lücke im bisherigen System füllen sowie die Einbindung ethischer Prinzipien in Entscheidungsprozesse begleiten und weiter fördern. Die bestehenden Strukturen, namentlich der Nationale Forschungsrat Thailands, hat nicht die Aufgabe und die Möglichkeit, sich hier zu positionieren.

Die Beispiele aus den Niederlanden und Thailand illustrieren die Herausforderungen auf dem Weg zu einer gerechten, universellen Gesundheitssicherung. In beiden Fällen gab es bereits existierende Strukturen im Gesundheitswesen deren Umgestaltung ein hohes Maß an politischem Willen voraussetzte. Positiv fällt vor allem die gesamtgesellschaftliche Beteiligung in Form von einkommensabhängigen Steuerbeträgen auf sowie die Versicherung unabhängig vom Gesundheitsstatus des Einzelnen als große Gemeinsamkeiten der Länder und in den Augen der Autoren für Gerechtigkeit sorgende Elemente auf. Unabhängig davon, ob sich Staaten auf eine Stärkung staatlicher oder privatwirtschaftlicher Institutionen konzentrieren, braucht es zur Verhinderung von Willkür und Lobbyismus klare Entschei-

39 Vgl. Thammatacharee et al. (2012).

40 Vgl. Tangcharoensathien et al. (2010) und Pagaiya/Noree (2009). 
dungskriterien und einen transparenten, verlässlichen Prozess zur Ressourcenverteilung. Hierbei können und sollten Ethikkommissionen und insbesondere existierende Strukturen wie Nationale Ethikräte als Garanten für einen gerechten Weg zu einer Universellen Gesundheitssicherung fungieren.

\section{LITERATUR}

Baltussen, Rob/Niessen, Louis (2006): »Priority setting of health interventions. The need for multi-criteria decision analysis «, in: Cost Effectiveness and Resource Allocation 4, 14 (2006).

Baltussen, Rob/Youngkong, Sitaporn/Paolucci, Francesco/Niessen, Louis (2010): »Multi-criteria decision analysis to prioritize health interventions: Capitalizing on first experiences«, in: Health Policy 96, 3 (2010), 262-264.

CCNE (1998): Technical progress, health and societal models. The ethical dimension of collective choices, Online: www.ccne-ethique.fr/sites/de fault/files/publications/avis057en.pdf [09.01.2016]

CCNE (2003): »Disparity in access to health care and participation in research on a global level - ethical issues. National Consultative Ethics Committee for Health and Life Sciences«, Online: www.ccneethique.fr.sites/default/files/publications/avis078en.pdf [19.04.2014].

CEG (2005): Ethical Aspects of cost-utility analysis. The Netherlands Centre for Ethics and Health, Online: www.ceg.nl/en/publications/ethicalaspects-of-cost-utility-analysis-2005 [09.01.2016].

CEG (2007a): »Appropriate evidence. Ethical questions concerning the use of evidence in health care policy«, Online: www.ceg.nl/uploads/publi caties/Appropriate_evidence_website.pdf [19.04.2014].

CEG (2007b): »Economisation of health care and professional ethics«, Online: www.ceg.nl/uploads/publicaties/Economisation_of_HC_and_ professional_ethics.pdf [19.04.2014].

CEG (2009): »Dilemmas of nurses and carers«, Online: www.ceg.nl/up loads/publicaties/Summary_Dilemmas_of_nurses_and_carers.pdf [19.04. 2014].

CNECV (2012): Opinion on a decision model for financing the costs of medicines. National Council of Ethics for the Life Sciences, Lisbon. 
Daniels, Norman (2000): »Accountability for reasonableness«, in: British Medical Journal 321, 7272 (2000), 1300-1301.

Daniels, Norman/Sabin, James E. (2008): »Accountability for reasonableness: an update«, in: British Medical Journal 337 (2008), a1850, 904905.

Den Exter, André (2010): »Health system reforms in the Netherlands: from public to private and its effects on equal access to health care«, in: European Journal of Health Law 17 (2010), 223-233.

Det Etiske Råd (1996): Prioritering i sundhedsvœsenet - en redegørelse, Copenhagen: KJ Bogtryk.

Deutscher Ethikrat (2011): Medical benefits and costs in healthcare. The normative role of their evaluation, Deutscher Ethikrat, Online: www. ethikrat.org/files/der-opinion-medical-benefits.pdf [09.01.2016].

Evans, Timothy/Chowdhury, A.Mushtaque/Evans, David B./Fidler, Armin H./Lindelow, Magnus/Mills, Anne/Scheil-Adlung, Xenia (2012): Thailand's Universal Coverage Scheme: Achievements and Challenges, Nonthaburi, Thailand: Health Insurance System Research Office.

Frewer, Andreas/Reis, Andreas/Bergemann, Lutz (Hg.) (2014): Gute oder vergütete Behandlung? Ethische Fragen der Gesundheitsökonomie. Jahrbuch Ethik in der Klinik (JEK), Bd. 7. Würzburg: Königshausen \& Neumann.

Friedman, Alex (2008): »Beyond accountability for reasonableness «, in: Bioethics 22, 2 (2008), 101-112.

Gruskin, Sofia/Daniels, Norman (2008): »Process is the point: justice and human rights: priority setting and fair deliberative process «, in: American Journal of Public Health 98, 9 (2008), 1573-1577.

Heinicke, Christina/Eriksson, Lotta/Saxena, Abha/Reis, Andreas (2014): »Universal Health Coverage. Basic Facts and the Role of National Ethics/Bioethics Committees«, in Frewer et al. (2014), 279-302.

Hermans Herbert/den Exter, André (1998): »Priorities and priority-setting in health care in the Netherlands«, in: Croatian Medical Journal 39 (1998), 346-355.

Kapiriri, Lydia/Norheim, Ole Frithjof/Martin, Douglas K. (2007): »Priority setting at the micro-, meso- and macro-levels in Canada, Norway and Uganda«, in: Health Policy 82, 1 (2007), 78-94.

Kapiriri, Lydia/Norheim, Ole Frithjof/Martin, Douglas K. (2009): »Fairness and accountability for reasonableness. Do the views of priority setting 
decision makers differ across health systems and levels of decision making?«, in: Social Science \& Medicine 68, 4 (2009), 766-773.

Kerstein, Samuel J./Bognar, Greg (2010): „Complete lives in the balance«, in: American Journal of Bioethics 10, 4 (2010), 37-45.

Limwattananon, Supon/Tangcharoensathien, Viroj/Prakongsai, Phusit (2010): »Equity in maternal and child health in Thailand«, in: Bullet of the World Health Organization 88 (2010), 420-427.

Maluka, Stephen/Kamuzora, Peter/Sansebastián, Miguel et al. (2011): »Implementing accountability for reasonableness framework at district level in Tanzania: a realist evaluation «, in: Implementation Science 6, 11 (2011). doi:10.1186/1748-5908-6-11 [1-15].

Martin, Douglas K./Giacomini, Mita/Singer, Peter A. (2002): »Fairness, accountability for reasonableness, and the views of priority setting decision-makers«, in: Health Policy 61 (2002), 279-290.

Okma, Kieke G./Crivelli, Luca (2013): »Swiss and Dutch >consumer-driven health care «: ideal model or reality?«, in: Health Policy 109 (2013), 105-112.

Ooms, Gorik/Latif, Laila A./Waris, Attiya/ Brolan, Claire E./Hammonds, Rachel/Friedman, Eric A./ Mulumba, Moses/Forman, Lisa (2014): »Is universal health coverage the practical expression of the right to health care?«, in: BMC International Health and Human Rights 14, 3 (2014), $1-7$.

Pagaiya, Nonglak/Noree, Thinakorn (2009): Thailand's Health Workforce: A Review of Challenges and Experiences, Health, Nutrition and Population (HNP) Discussion Paper, Human Development Network, The World Bank.

Peacock, Stuart/Mitton, Craig/Bate, Angela/McCoy B./ Donaldson C. (2009): »Over-coming barriers to priority setting using interdisciplinary methods«, in: Health Policy 92, 2-3 (2009), 124-132.

Persad, Govind C./Wertheimer, Alan/Emanuel, Ezekiel J. (2009): »Principles for allocation of scarce medical interventions «, in: The Lancet 373, 9661 (2009), 423-431.

Persad, Govind C./Wertheimer, Alan/Emanuel, Ezekiel J. (2010): »Standing by our principles: meaningful guidance, moral foundations, and multi-principle methodology in medical scarcity «, in: American Journal of Bioethics 10, 4 (2010), 46-48. 
Schäfer, Willemijn/Kroneman, Madelon/Boerma, Wienke/van den Berg, Michael/ Westert, Gert/ Devillé, Walter/van Ginneken, Ewout. (2010): »The Netherlands: health system review«, in: Health System in Transition 12, 1 (2010), 1-228.

Somkotra, Tewarit/Lagrada, Leizel P. (2009). »Which households are at risk of catastrophic health spending: experience in Thailand after universal coverage«, in: Health Affairs 28, 3 (2009), 467-478.

Tangcharoensathien, Viroj/Pitayarangsarit, Siriwan/Patcharanarumol, Walaiporn/Prakongsai, Phusit/ Sumalee, Hathaichanok/Tosanguan, Jiraboon/Mills, Anne (2013): »Promoting universal financial protection: how the Thai universal coverage scheme was designed to ensure equity. In: Health Research Policy and Systems 11 (2013).

Tangcharoensathien, Viroj/Swasdiworn, Wina,/Jongudomsuk Pongpisut/ Srithamrongswat, Samrit/ Patcharanarumol, Walaiporn/Prokongsai, Phusit/Thammathataree, Jadej (2010): Universal Coverage Scheme in Thailand: Equity Outcomes and Future Agendas to Meet Challenges, World Health Report, Background Papers, No 43.

Thammatacharee, Noppakun/Tisayaticom, Kanjana/Suphanchaimat, Rapeepong/Limwattananon, Supon/Putthasri, Weerasak/Netsaengtip, Rajana/ Tangcharoensathien, Viroj (2012): »Prevalence and profiles of unmet healthcare need in Thailand «, in: BMC Public Health 12 (2012), 923.

United Nations General Assembly (2012): »United Nation General Assembly Resolution A/67/L.36, New York«, Online: http://www.un.org/ga/search/viewm_doc.asp?symbol=A /67/L.36 [19.04.2014].

United Nations (1948): Resolution der Generalversammlung A/RES/217 A (III): Allgemeine Erklärung der Menschenrechte, Paris, 10.12.1948 Online: www.un.org/depts/german/menschenrechte/aemr.pdf [29.12.2015]. United Nations (2000a): General Comment No 14 »The right to the highest attainable standard of health«, CESCR E/C.12/2000/4, 11.08.2000. Online: www.ohchr.org/Documents/Issues/Women/WRGS/Health/GC 14.pdf [24.11.2015].

United Nations (2000b): United Nation General Assembly Resolution A/55/ L.2 Millennium Declaration, New York, 18.09.2000, Online: www.un. org/millennium/declaration/ares552e.pdf [29.12.2015].

United Nations (2015): Millenium Development Goals Report 2015, New York 2015, Online: www.ua.undp.org/cotent/dam/undp/library/MDG/ english/UNDP_MDG_Report_2015.pdf [29.12.2015]. 
Van de Wetering, E. J./Stolk, E. A./van Exel, N. J./Brouwer, Werner B. F. (2013): »Balancing equity and efficiency in the Dutch basic benefits package using the principle of proportional shortfall «, in: The European Journal of Health Economics 14, 1 (2013), 107-115.

WHO (2005): Sustainable health financing, universal coverage and social health insurance. WHA 58.33, Fifty-Eighth World Health Assembly. Geneva, 16-25 May 2005, 124-126., Online: http://apps.who.int-/ gb/ebwha/pdf_files/WHA58-REC1/english/A58_2005_REC1-en.pdf [19.04.2014].

WHO (2010): The World Health Report 2010. Health systems financing. The path to universal coverage. Geneva: WHO Press.

WHO (2013): World Health Report 2013. Research for universal health coverage, Geneva: WHO Press.

WHO (2014a): What is universal coverage, Online: www.who.int/ health_financing/universal_coverage_definition/en/ [19.04.2014].

WHO (2014b): Making fair choices on the path to universal health coverage. Final report of the WHO Consultative Group on Equity and Universal Health Coverage, Geneva: WHO Press.

WHO (2014c): Twelfth general programme of work 2014-19: Not merely the absence of disease, Geneva: WHO Press.

Youngkong, Sitaporn/Baltussen, Rob/Tantivess, Sripen/Mohara, Adun/ Teerawattananon, Yot (2012): »Multicriteria decision analysis for including health interventions in the universal health coverage benefit package in Thailand«, in: Value in Health 15, 6 (2012), 961-970. 


\section{Ambivalenzen der Medikalisierung}

Ein Plädoyer für das Ernstnehmen der subjektiven

Perspektive im Umgang mit Gesundheit und Krankheit

MARTINA SCHMIDHUBER

\section{HINFÜHRUNG}

In Artikel 12 des Internationalen Paktes über wirtschaftliche, soziale und kulturelle Menschenrechte (ICESCR, kurz: UN-Sozialpakt) von 1966 wird »das Recht eines jeden auf das für ihn erreichbare Höchstmaß an körperlicher und geistiger Gesundheit« anerkannt. Das bedeutet aber nicht, dass jeder Mensch ein Recht darauf haben kann, stets gesund zu sein - dies hängt von vielen Faktoren ab, u.a. von der eigenen körperlichen und geistigen Grundkonstitution. ${ }^{1}$ Vielmehr geht es im UN-Sozialpakt um das Recht eines jeden auf einen Zugang zu einer bestehenden und sich entwickelnden

1 Dazu erläutert der UN-Ausschuss für wirtschaftliche, soziale und kulturelle Rechte in seinem Kommentar zum Recht auf Gesundheit (CESCR E/C.12/ 2000/4, 11. August 2000, Ziff. 8): »The right to health is not to be understood as a right to be healthy. The right to health contains both freedoms and entitlements. The freedoms include the right to control one's health and body, including sexual and reproductive freedom, and the right to be free from interference, such as the right to be free from torture, non-consensual medical treatment and experimentation. By contrast, the entitlements include the right to a system of health protection which provides equality of opportunity for people to enjoy the highest attainable level of health.« 
Infrastruktur des Gesundheitswesens. Deshalb werden im zweiten Teil des Artikels 12 die zu unternehmenden Schritte der Vertragsstaaten zur vollen Verwirklichung dieses Rechts festgelegt. Dazu zählen erforderliche Maßnahmen:

a. zur Senkung der Zahl der Totgeburten und der Kindersterblichkeit sowie zur gesunden Entwicklung des Kindes;

b. zur Verbesserung aller Aspekte der Umwelt- und der Arbeitshygiene;

c. zur Vorbeugung, Behandlung und Bekämpfung epidemischer, endemischer, Berufs- und sonstiger Krankheiten;

d. zur Schaffung der Voraussetzungen, die für jedermann im Krankheitsfall den Genuss medizinischer Einrichtungen und ärztlicher Betreuung sicherstellen.

Darüber hinaus wird in der Allgemeinen Erklärung der Menschenrechte (AEMR) - die zusammen mit dem UN-Sozialpakt und dem Internationalen Pakt über bürgerliche und politische Rechte (ICCPR, kurz: UN-Zivilpakt) als internationaler Menschenrechtskodex gilt - im Artikel 25, Ziffer 1 ein Lebensstandard, der Gesundheit befördert, festgelegt:

»Jeder hat das Recht auf einen Lebensstandard, der seine und seiner Familie Gesundheit und Wohl gewährleistet, einschließlich Nahrung, Kleidung, Wohnung, ärztliche Versorgung und notwendige soziale Leistungen, sowie das Recht auf Sicherheit im Falle von Arbeitslosigkeit, Krankheit, Invalidität oder Verwitwung, im Alter sowie bei anderweitigem Verlust seiner Unterhaltsmittel durch unverschuldete Umstände.«

Mangelernährung, keine angemessene Kleidung, schimmlige Wohnungen, krank machende Arbeitsbedingungen und fehlender Zugang zu ärztlicher Versorgung lassen Menschen auch bei guter Grundkonstitution erkranken. Insofern ist es erforderlich, dass die Voraussetzungen für Gesundheit und der Zugang zu einem Gesundheitssystem bei Krankheit menschenrechtlich gewährleistet werden.

Was aber ist eigentlich unter Gesundheit zu verstehen? Mit dieser Frage haben sich schon viele Autoren beschäftigt und unterschiedliche Antworten 
gegeben. ${ }^{2}$ Die meisten Positionen lassen sich entweder der objektiven oder der subjektiven Theorie zuordnen. ${ }^{3}$ So hat beispielsweise Christopher Boorse in den 1970er Jahren eine Biostatistische Theorie entwickelt, in der ein naturalistischer bzw. objektiver Gesundheitsbegriff zugrunde gelegt wird. Er geht dabei von einer statistisch normalen Funktionsfähigkeit des Organismus aus, die als gesund gilt. ${ }^{4}$ Als Vertreter der entgegengesetzten Position zu Boorse kann Lennart Nordenfelt genannt werden, der eine subjektive Theorie von Gesundheit einnimmt. Nordenfelt versteht Gesundheit als die Fähigkeit zur Erfüllung individueller Präferenzen. ${ }^{5}$ Norman Daniels wiederum schlägt einen objektiven Begriff von Gesundheit vor, der möglichst für alle Menschen gelten soll, und verknüpft diesen mit individuellem Wohlergehen. ${ }^{6}$

Im folgenden Beitrag soll die Frage, ob Gesundheit und Krankheit objektiv oder subjektiv betrachtet werden müssen, anhand von Beispielen, die gegenwärtig besondere gesellschaftliche Brisanz aufweisen, untersucht werden. Dabei soll dem Leiden an einem Zustand besondere Beachtung geschenkt werden. Anschließend soll gefragt werden, was daraus menschenrechtlich für den Lebensstandard, der Gesundheit und Wohl ermöglichen soll, folgt. Wenn Gesundheit bzw. Krankheit objektiv definiert werden, könnte es sein, dass jemand als krank gilt, der sich aber gesund fühlt. Umgekehrt könnte sich jemand aufgrund eines Leidens krank fühlen, aber aus gesellschaftlicher Sicht nicht als krank gelten.

Im vorliegenden Text wird dafür plädiert, die subjektive Perspektive ernst zu nehmen, auch wenn eine objektive Sichtweise in vielen Fällen durchaus Sinn macht. Die objektive Perspektive kann stets einen gewissen Rahmen zur Verfügung stellen, innerhalb dessen die subjektive Sicht berücksichtigt werden muss, sodass die Betroffenenperspektive nicht zu kurz kommt. Ansonsten besteht die Gefahr, Krankheitskonzepte über Individuen zu stülpen, die sich damit nicht identifizieren können. Diejenigen, die von

2 Vgl. dazu auch die Überlegungen in Rothhaar/Frewer (2012).

3 An anderen Stellen werden die unterschiedlichen Theorien sehr ausführlich diskutiert, vgl. z.B. Friedrich (2014). Für den Zweck des vorliegenden Beitrags werden sie hier nur in aller Kürze erwähnt.

4 Vgl. Boorse (1977), 542-573.

5 Vgl. Nordenfelt (1995); vgl. auch Nordenfelt (2012), 89-104.

6 Vgl. Daniels (2008). 
etwas betroffen sind, was objektiv als Krankheit bezeichnet wird, sollten also - so das Plädoyer - von der Gesellschaft nicht als krank bezeichnet werden, nur weil sie gewissen Normen nicht entsprechen. Wie in den Beispielen noch deutlich werden sollte, spielt auch die Kategorie des »Intersubjektiven« eine wesentliche Rolle. Denn nicht nur das subjektive Empfinden und das objektiv medizinisch Feststellbare beeinflussen die Auffassung, was als krank gilt, sondern auch das, was von einer Gesellschaft an den Einzelnen an Erwartungen herangetragen wird.

In unserem Alltagssprachgebrauch scheint relativ klar zu sein, was die Begriffe Gesundheit und Krankheit bedeuten. Wenn jemand krank ist, beispielsweise an einem grippalen Infekt leidet, fühlt er sich nicht gut und wird für diese Zeit von der Pflicht, zur Arbeit zu gehen, entbunden. Die Zuschreibung von Krankheit hat demnach normative Relevanz. ${ }^{7}$ Dennoch gibt es für den sozialrechtlichen Krankheitsbegriff kein klar definiertes Konzept. Wann jemand in welchem Umfang sozialrechtliche Leistungsansprüche hat, ist nicht klar bestimmt. Die Rede ist von einem regelwidrigen Körper- oder Geisteszustand, der ärztlicher Behandlung bedarf. ${ }^{8}$ Unter anderem wegen dieser Unschärfe und großen Interpretationsmöglichkeiten stellen sich angesichts aktueller Entwicklungen Fragen, die nicht so leicht zu beantworten sind: Ist ungewollte Kinderlosigkeit eine Krankheit? Wenn keine Kinder gezeugt werden können, scheint ja etwas im Körper regelwidrig zu sein. Ist Altern eine Krankheit, gegen die wir mittels Anti-AgingMedizin ankämpfen müssen? Ist Alzheimer-Demenz eine Krankheit, die uns vor schwer zu bewältigende Herausforderungen stellt oder ein Teil des Lebens, mit dem wir in unserer immer älter werdenden Gesellschaft zurechtkommen müssen? Diese vergleichsweise neuen Fragen machen deutlich, dass längst nicht klar ist, wo die Grenze zwischen Gesundheit und Krankheit zu ziehen ist. Medizin-technische Entwicklungen eröffnen neue Wege, in den Körper einzugreifen und auf diese Weise Wünsche mit Hilfe

7 Thomas Schramme konstatiert in diesem Zusammenhang »wichtige normative Implikationen«, die die Zuschreibung von Krankheit hat. Vgl. Schramme (2012b), 9.

8 Vgl. Schweda/Marckmann (2012), 181. 
von Medizin zu erfüllen, ${ }^{9}$ wie z.B. den Kinderwunsch in nicht mehr reproduktionsfähigem Alter.

Viele Lebensbereiche, die lange nicht als Felder der Medizin galten, werden nun medikalisiert, d.h. zu einem Thema der Medizin gemacht, und in diesem Zuge mit Krankheit in Verbindung gebracht. ${ }^{10}$ Es stellt sich jedoch die Frage, ob nicht subjektives Leid eine maßgeblichere Rolle für die Anerkennung von Krankheit haben sollte. Wenn jemand krank ist, hat er ein Recht auf Versorgung. Hat er das nur, wenn er von Medizinern für krank erklärt wird, oder auch, wenn er sich krank fühlt? Oder umgekehrt: Wenn jemand z.B. aufgrund einer Beeinträchtigung für krank erklärt wird, aber nicht leidet und sich nicht krank fühlt, kann es dann trotz Patientenautonomie geboten sein, sich einer Behandlung zu unterziehen? ${ }^{11}$

Anhand von drei Beispielen soll dem subjektiven Leiden als Parameter für Gesundheit und Krankheit nachgegangen und anschließend versucht werden, zu klären, was daraus für das Menschenrecht auf einen Lebensstandard, der Gesundheit gewährleistet, folgt. Es gilt zunächst zu berücksichtigen, dass es Krankheiten gibt, die (noch) kein Leiden verursachen, weil man beispielsweise (noch) nichts davon weiß. So ließe sich vielleicht schon jetzt eine genetisch bedingte Erkrankung diagnostizieren, die aber erst zu einem späteren Zeitpunkt ausbrechen wird. Nur das Nicht-Wissen von dieser bereits schlummernden Krankheit vermeidet Leiden. Aber man könnte sich mittels prädiktiver Genetik testen lassen. Diese ermöglicht es, kausal wirksame genetische Veränderungen festzustellen, sodass für einen kleinen Teil von monogenetisch bedingten Erkrankungen ein sicherer Krankheitsausbruch prognostiziert werden kann. Für viele andere Fälle können jedoch nur Wahrscheinlichkeitsaussagen getroffen werden. ${ }^{12}$ Das bedeutet also, dass man zwar damit rechnen muss, eines Tages an einer

9 Zur wunscherfüllenden Medizin vgl. Eichinger (2012), 140-143; Eichinger (2013), v.a. 212-223.

10 Ivan Illich prägte in den 1970er-Jahren den Begriff »Medikalisierung «, vgl. Illich (1975). Für neueste Debatten zur Medikalisierung vgl. z. B. Birnbacher (2014), 203, Mayer-Olkin (2015) und Wardrope (2015).

11 In der Psychiatrie ist dieses Thema ein spezielles Problem, weil es um die Frage der vorhandenen oder eben nicht vorhandenen Einwilligungsfähigkeit zu einer Behandlung geht, vgl. dazu Schramme (2000).

12 Vgl. Schöne-Seifert (2007), 96-97. 
genetischen Krankheit zu leiden, dies aber nicht unbedingt zutreffen muss. ${ }^{13}$ Möglicherweise erzeugt aber gerade diese Wahrscheinlichkeit und Unsicherheit großes Leid. ${ }^{14}$ Hätte man sich nie auf die prädiktive Genetik eingelassen, wüsste man gar nicht, dass man möglicherweise z.B. die Huntingtonsche Erkrankung bekommen könnte, und würde auch noch kein Leid verspüren, bevor man überhaupt erkrankt ist. ${ }^{15}$

Abgesehen von diesen Fällen gibt es Beispiele, an denen sich zeigen lässt, dass Leiden entsteht, aber nicht klar ist, ob es sich aus gesellschaftlicher Sicht tatsächlich um eine Krankheit handelt. Exemplarisch sollen drei Themen von besonderer aktueller gesellschaftlicher Relevanz betrachtet werden: Kinderlosigkeit, Altern und Alzheimer-Demenz. Inwiefern verursachen diese Zustände aus subjektiver Sicht Leid? Inwieweit spielen dabei die intersubjektiven resp. gesellschaftlichen Einflüsse eine Rolle? Ist das subjektive Leiden ein Grund, sie als Krankheiten zu bezeichnen und menschenrechtliche Ansprüche geltend zu machen?

\section{DREI BEISPIELE}

\subsection{Kinderlosigkeit}

Reproduktionsmedizin wird von manch kritischer Stimme als »bloße LifeStyle-Medizin ${ }^{16}{ }^{6}$ bezeichnet. In unserer Welt, in der alles machbar und herstellbar zu sein scheint, würden nun auch Kinder als Teil des Lebensstils

13 Vgl. auch Lemke (2000), 246.

14 Wie Lemke am Beispiel der USA zeigt, muss darüber hinaus auch mit Formen der Diskriminierung gerechnet werden: „So wird etwa Ehepaaren die Adoption von Kindern untersagt, wenn bei einem der Elternteile eine Disposition für eine genetische Krankheit vorliegt. [...] Es kommt auch vor, dass Kranken- und Lebensversicherungen Verträge kündigen oder deren Abschluss verweigern, wenn bei ihren (potenziellen) Kunden durch Gentests eine Krankheitsdisposition festgestellt wurde.« Lemke (2000), 246-247.

15 Als Vorteil der prädiktiven Genetik wird v.a. die bewusstere Lebensplanung genannt. Vgl. Schöne-Seifert (2007), 97.

16 Anselm kritisiert diese einseitige, zu kurz greifende Sicht: Anselm (2013), 97. 
verstanden werden. ${ }^{17}$ Mehr als fünf Millionen Kinder wurden bereits mithilfe der In-Vitro-Fertilisation (IVF) geboren. ${ }^{18}$ Kinder, so konstatiert beispielsweise der Medizinethiker Giovanni Maio, müssten aber als »Gabe und Geheimnis « ${ }^{19}$ verstanden werden und dürften nicht die Vorstellung der Bestellbarkeit wecken. Andererseits betont Maio aber auch, dass er die Not kinderloser Paare nicht bagatellisieren möchte. ${ }^{20}$ Es scheint also selbst für jene, die reproduktionsmedizinische Maßnahmen sehr kritisch sehen, klar zu sein, dass Menschen an Kinderlosigkeit leiden können und es sich beim Einsatz von reproduktionsmedizinischen Maßnahmen nicht in allen Fällen um unreflektierte Life-Style-Medizin handelt. Aber bedeutet dieses mögliche Leiden an Kinderlosigkeit, dass es sich um eine Krankheit handelt? ${ }^{21}$ Es gibt einige Anzeichen dafür, dass Kinderlosigkeit aufgrund körperlicher Fehlfunktionen als Krankheit einzustufen ist. So z.B. die Tatsache, dass die Kosten der ersten drei IVF-Versuche von den Krankenkassen teilweise bis zu 100 Prozent übernommen werden. ${ }^{22}$ Auch nach Boorses biostatistischer Theorie ist Reproduktion ein wichtiges Merkmal für einen gesunden Organismus. ${ }^{23}$ Aber auch Aussagen wie jene des Theologen Rainer Anselm, der den Wunsch nach eigenen Kindern als »etwas ganz Normales « ${ }^{24}$ bezeichnet, machen deutlich, dass offenbar eine Norm nicht erfüllt bleibt, wenn jemand ungewollt kinderlos bleibt. Insofern wäre es aus Anselms Sicht selbstverständlich, dass unerwünschte Kinderlosigkeit als Krankheit wahrgenommen werden kann. ${ }^{25}$ Wenn Kinderlosigkeit als Krankheit bezeichnet wird, zeigt dies sehr deutlich, dass von einer Normabweichung ausgegan-

17 Vgl. Maio (2013), 37.

18 Vgl. Beck-Gernsheim (2013), 337.

19 Maio (2013), 33.

20 Vgl. ebd.

21 Von der WHO wird ungewollte Kinderlosigkeit als Krankheit anerkannt.

22 Vgl. www.wunschkinder.net/aktuell/gesellschaft/krankenkassen/kunstlichebefruchtung-welche-krankenkassen-zahlen-mehr-5265/ [21.10.2014].

23 Vgl. Boorse (1977), 549-550.

24 Anselm (2013), 97.

25 Vgl. Anselm (2013), 97. Anselm kommt jedoch zu dem Schluss, »dass ungewollte Kinderlosigkeit aus gesellschaftlicher Perspektive zwar als soziale Aufgabe, nicht aber als Krankheit zu verstehen« ist. Vgl. Anselm (2013), 111. 
gen wird und dass von gesellschaftlicher Seite Reproduktionsfähigkeit erwartet wird, der eventuell nachgeholfen werden muss.

Betrachtet man Kinderlosigkeit aus subjektiver Sicht, kann diese Leiden verursachen. Mit dem Leid, das aufgrund ungewollter Kinderlosigkeit entsteht, kann sehr unterschiedlich umgegangen werden. So gibt es Menschen, die zwar an ihrer Kinderlosigkeit leiden, sich aber dennoch keinen reproduktionsmedizinischen Maßnahmen unterziehen. Sie versuchen stattdessen, ihrem Leben andere Inhalte zu geben. Sie fühlen sich nicht unbedingt krank und fassen es vielleicht als Schicksal oder Gottes Wille auf, dass sie keine eigenen Kinder zeugen können. Jene Menschen, die daran leiden und ihren Kinderwunsch unbedingt umsetzen wollen, haben in unserer Gesellschaft die Option, Reproduktionsmedizin in Anspruch zu nehmen. Es werden Möglichkeiten geboten, die das subjektive Wohl, das für viele Menschen mit Elternschaft verbunden ist, befördern - auch wenn es freilich kein Recht auf Elternschaft durch Reproduktionsmedizin geben kann und auch keine Garantie, tatsächlich schwanger zu werden. ${ }^{26}$

Kinderlosigkeit kann mit Leiden verbunden sein, aber nicht alle von Kinderlosigkeit Betroffenen fühlen sich krank und behandlungsbedürftig. Das zeigt, wie subjektiv diese Empfindung ist. Deshalb wäre es unangemessen, Kinderlosigkeit von vornherein in einer Gesellschaft als Krankheit zu definieren, weil damit dieser höchst private Lebensbereich medikalisiert würde. Es könnte sozialer Druck entstehen, etwas gegen ungewollte Kinderlosigkeit zu tun, wenn diese generell als Krankheit gewertet würde. Umgekehrt soll aber subjektives Leid bei Kinderlosigkeit ernst genommen werden. Mit Hilfe von erfolgreich durchgeführter Reproduktionsmedizin kann ein Lebensstandard erreicht werden, der den Betroffenen Wohl und Gesundheit ermöglicht.

26 Die Erfolgsquote reproduktionsmedizinischer Maßnahmen beträgt 15,4 Prozent. Es handelt sich dabei um die sogenannte baby-take-home-rate. Das bedeutet, dass viele Paare trotz Reproduktionsmedizin kinderlos bleiben. Vgl. Maio (2013), 14. 


\subsection{Altern}

Auch am Altern und an ihrer Sterblichkeit scheinen Menschen immer mehr zu leiden - allerdings sogar vollkommen unabhängig von Alterskrankheiten. Altern ist ein lebenslanger Prozess, der mit der Geburt beginnt und mit dem Tod endet. Üblicherweise sprechen wir jedoch dann vom Altern, wenn schon ein gewisses Lebensalter erreicht ist. "Das Alter « steht für eine Lebensphase. Wann jedoch die Lebensphase des Alters eintritt, wird gesellschaftlich bestimmt. ${ }^{27}$ In dieser Hinsicht lässt sich ein gesellschaftlicher Wandel konstatieren: Menschen, die noch vor 30 Jahren bereits als alt galten, gelten heute nicht mehr als alt. Das Alter, so scheint aktuell ein gesellschaftliches Postulat zu lauten, darf man sich nicht mehr anmerken lassen. Ähnlich wie bei der Reproduktion zeigen sich also auch beim Alter intersubjektive Erwartungshaltungen, die der Einzelne häufig internalisiert und versucht umzusetzen: Man muss alles dafür tun, um lange jung und fit zu sein oder vielmehr so zu wirken. ${ }^{28}$

Ihr eigenes Altern wird Menschen meistens erst ab einer gewissen Lebensphase bewusst: mit den ersten körperlichen Veränderungen, wie Falten, der Abnahme der körperlichen Leistungsfähigkeit und dem Wahrnehmen der Endlichkeit, das impliziert, dass die eigenen Möglichkeiten während der Lebenszeit begrenzt sind. ${ }^{29}$ Und genau diese Veränderungen, die die Zeit mit sich bringt, können Leid hervorrufen. ${ }^{30}$ Dennoch würde man nicht behaupten, dass Altern per se eine Krankheit ist. ${ }^{31}$ Vielmehr zeigt sich im Rahmen der Medikalisierung des Alters, was sein soll und was nicht sein soll. Über die Gesellschaft vermittelte Vorstellungen von "gutem Altern" beeinflussen die Individuen erheblich. Mittels Botox, Vitaminbehandlungen ohne Krankheitsbezug bis hin zu Straffungen verschiedener Körperpartien wird das Bild des jungen, leistungsfähigen Menschen als Norm propagiert.

In Biotechnologie und Robotik wird an dem schon seit jeher bestehenden Traum von ewiger Jugend und Unsterblichkeit gearbeitet. ${ }^{32}$ Es wird

27 Vgl. Kruse/Wahl (2010), 7.

28 Vgl. Maio (2012), 16.

29 Vgl. auch Steinfath (2001), 286.

30 Vgl. auch Bozzaro (2012), 219-248 und Bozzaro (2014).

31 Vgl. Schramme (2009), 235-263.

32 Vgl. dazu Krämmer/Schmidhuber (2015), 29-48. 
versucht, mittels moderner Technologien Altern und Sterblichkeit zu verhindern. ${ }^{33}$ Diese rasanten Entwicklungen, die von Trans- und Posthumanisten vorangetrieben werden, verändern unser Menschenbild grundlegend. ${ }^{34}$ Denn diese Strömungen machen es sich zum Ziel, den menschlichen Körper und die menschliche Psyche mit allen vorhandenen technischen und medizinischen Interventionen zu verbessern. ${ }^{35}$ Ziele sind höhere Intelligenz, Resistenz gegenüber Erkrankungen und dem Alterungsprozess bis hin zur Überwindung des Todes. ${ }^{36}$ Im Grunde handelt es sich hierbei um Enhancement. ${ }^{37}$ Die Verschönerung, Verbesserung und Leistungssteigerung bei gesunden Menschen wird angestrebt. Viele medizinische Möglichkeiten, die ursprünglich zu therapeutischen Zwecken entwickelt wurden, zeigen auch Enhancement-Wirkung: Medikamente, die zur Steigerung der Aufmerksamkeit oder zur Verbesserung des Gedächtnisses führen, Medikamente zur Stimmungsaufhellung oder zur Senkung des Schlafbedarfs werden schon jetzt bei gesunden Personen eingesetzt. ${ }^{38}$

Ähnlich wie am Beispiel der Kinderlosigkeit wird deutlich, dass Altern bei Menschen aus subjektiver Sicht durchaus Leiden verursachen kann, dass Altern aber nicht von vornherein mit Krankheit gleichgesetzt werden darf. Denn Menschen, die am Alter(n) nicht leiden, sondern es als wichtigen Prozess in ihrem Leben sehen, der auch positive Seiten mit sich bringt, und die deshalb auch keine Anti-Aging-Maßnahmen in Anspruch nehmen, sind nicht krank, sondern gehen lediglich anders mit dem Alter um. Insofern ist es auch hier erforderlich, das subjektive Leid von einer medizinischen Definition zu trennen. Altern als krank zu bezeichnen, würde implizieren, dass dieser Prozess einer Veränderung bedarf. Wer tatsächlich subjektiv an seinem Alter leidet, hat gegenwärtig - und, wie deutlich wurde, in

33 Vgl. Lacina (2009), 101.

34 Vgl. dazu Heil (2010), 127-149.

35 Vgl. Lacina (2009), 102.

36 Vgl. ebd.

37 Vgl. Schöne-Seifert (2007), 99-100 und Eissa (2014).

38 An diese Stelle ließe sich trefflich über Vor- und Nachteile von Enhancement diskutieren. Wer kann sich Enhancement leisten? Hier stellt sich die Gerechtigkeitsfrage. Sind dann wiederum jene am leistungsfähigsten und fittesten, die es sich finanziell leisten können? Ist es sinnvoll, körperliche Funktionen wie das Schlafbedürfnis auszuschalten? Vgl. dazu auch Eissa (2014). 
Zukunft vermutlich noch mehr - Möglichkeiten, dem Altern mittels technischer Eingriffe und Anti-Aging-Methoden entgegenzuwirken. Dies kann das subjektive Wohlergehen positiv beeinflussen. Aber auch dafür gibt es keine Garantie, denn die äußerlichen Veränderungen helfen nicht notwendigerweise, mit dem Alter besser zurecht zu kommen, denn es besteht die Gefahr, dass »ein an sich existenzielles Leiden somatisiert und dadurch in den Zuständigkeitsbereich der Medizin gebracht « ${ }^{39}$ wird. »Diese Verschiebung verkennt nicht nur die eigentliche Natur des Leidens, sondern kann auch als Zeichen der Unfähigkeit gedeutet werden, Leiden in einer anderen als der medizinisch-technischen Weise zu begegnen. « ${ }^{40}$ Die Auseinandersetzung mit sich selbst, der eigenen Biographie und dem persönlichen Identitätsverständnis wären mögliche Wege, um dem Alter etwas Positives abzugewinnen. Langlebigkeit ohne zu altern als Menschenrecht $\mathrm{zu}$ bezeichnen, wie dies der britische Altersforscher Aubrey de Grey konstatiert, $^{41}$ würde wohl aus Sicht vieler zu weit führen. Selbst wenn Menschen unter ihrem Alter leiden, wäre die Linderung dieses Leidens als Forderung an die Menschenrechte unverhältnismäßig. Dass Menschen im Alter sozial abgesichert sein sollten und versorgt werden, ist aber durchaus ein Menschenrecht, das jedoch nichts mit einem Recht auf Anti-AgingStrategien zu tun hat.

\subsection{Alzheimer-Demenz}

Anders als bei Kinderlosigkeit und Altern verhält es sich bei einer tatsächlich anerkannten Krankheit, die als Leid der Gesellschaft verstanden wird: der Alzheimer-Erkrankung. Im Jahre 2010 waren laut World Alzheimer Report weltweit 36 Millionen Menschen an Demenz erkrankt. Die Zahl der Erkrankungen wird sich laut Prognose im Jahr 2030 verdoppelt und 2050 voraussichtlich verdreifacht haben. ${ }^{42}$ Nach den Kriterien des ICD-10 ist das Demenzsyndrom durch ein spezifisches Symptommuster gekennzeichnet, welches sich auf kognitive Leistungen und nicht-kognitive Anzeichen bezieht. Aber selbst im Kontext dieser anerkannten Erkrankung gibt es

39 Bozzaro (2012), 246.

40 Ebd.

41 Vgl. de Grey (2005), 659-663.

42 Alzheimer's Disease International (2013), 1. 
Vertreter wie den streitbaren Soziologen Reimer Gronemeyer, die der Ansicht sind, dass Alzheimer-Demenz keine Krankheit sei. Demenz werde medikalisiert, es werde in diesem Zuge dafür gesorgt,

»dass Gelder von der Biomedizin aufgesaugt werden können, und gleichzeitig der Frage nach den sozialen Dimensionen der Demenz weiterhin keine Aufmerksamkeit gewidmet werden muss. « ${ }^{43}$

Nach Gronemeyer wäre es vielmehr notwendig, unsere gesellschaftlichen Werte zu hinterfragen, die vor allem an Geschwindigkeit, Automatisierung und Leistung gekoppelt sind. Denn Demenz sei vielleicht ein Zeichen unserer hektischen Zeit, »eine Art gerontologisches Burn-out «. ${ }^{44}$ Wäre unsere Welt gastfreundlicher, ${ }^{45}$ so Gronemeyer, wäre Demenz kein so großes Schreckgespenst, wir würden uns mit ihr als Teil des Alters abfinden.

Allerdings muss berücksichtigt werden, dass die Symptome der Alzheimer-Demenz den Betroffenen so einschränken, dass er immer weniger zur selbstständigen Lebensführung fähig ist. Zum anfänglichen Nachlassen des Kurzzeitgedächtnisses kommen u.a. Orientierungslosigkeit, Einschränkung der motorischen Fähigkeiten, Störungen des Schlaf-Wach-Rhythmus, Nachlassen des Langzeitgedächtnisses (die engsten Angehörigen werden in späteren Stadien oftmals nicht mehr erkannt) und Verlust der verbalen Fähigkeiten hinzu. In der letzten, schweren Phase der Erkrankung sind die Patienten meistens bettlägerig und müssen künstlich ernährt werden. Die Anfälligkeit für Infektionen steigt, deshalb sterben Menschen mit schwerer Demenz häufig an einer Lungenentzündung. ${ }^{46}$

Angesichts dieser Symptome, die bei anderen Erkrankungen durchaus als Krankheitssymptome gewertet werden, ist nur schwer zu bezweifeln, dass Demenz eine Krankheit ist. Die Pflege von Menschen mit Demenz in der Familie wird immer schwieriger, weil nur noch selten mehrere Generationen unter einem Dach leben, meistens sogar nicht einmal in derselben Stadt. Wenn sich Angehörige entschließen, einen Menschen mit Demenz in

43 Gronemeyer (2013), 43.

44 Ebd., 251.

45 Vgl. ebd., 260.

46 Vgl. Maier et al. (2011), 33-39. 
der Familie zu pflegen, ist dies mit großen Belastungen verbunden. ${ }^{47}$ Eine gastfreundliche Welt, in der auch Menschen mit körperlichen und geistigen Defiziten Platz finden, ist daher wünschenswert und wird auch zukünftig notwendig sein. Dennoch kann es auch Vorteile haben, eine Krankheit zu diagnostizieren und zu benennen, denn wenn die Symptome bzw. die kognitiven Störungen Krankheitswert erhalten, kann mit »krankheitsgerechtem Verhalten ${ }^{48}$ der Umgebung gerechnet werden, was für Betroffene wiederum entlastend wirken kann. ${ }^{49}$

Angesichts eines Zustandes, der objektiv als Krankheit wahrgenommen wird und auch meist von den Betroffenen, zumindest in den ersten Stadien, als Krankheit empfunden wird, scheint Gronemeyers Postulat, Demenz nicht als Krankheit aufzufassen, äußerst unplausibel. Hintergrund seiner Überlegungen ist jedoch, dass Menschen mit Demenz in die Gesellschaft integriert werden sollten. Aufgrund unserer schnelllebigen Welt entfernen wir uns von Menschen mit Demenz und empfinden ihre Langsamkeit als störend. Vielmehr wird intersubjektiv vermittelt, dass in unserer Gesellschaft alles schnell und reibungslos funktionieren muss; Ausreißer, die den Prozess stören, werden nicht akzeptiert. ${ }^{50}$

Es kann angenommen werden, dass die Angehörigen häufig stärker an der Erkrankung leiden als die Betroffenen selbst, so Gronemeyer. ${ }^{51}$ Das Leben, so wie es gewöhnlich abläuft, muss umgestellt und an den Erkrankten angepasst werden, und auch die Belastung durch die Pflege ist nicht zu unterschätzen. Die von Demenz Betroffenen hingegen vergessen im Laufe der Erkrankung, dass sie vergessen, und können im besten Fall intensiv im Augenblick leben. Deshalb wird der Punkt, ab dem das Vergessen vergessen wird, die "gnädige Schwelle« genannt. $^{52}$

47 Vgl. dazu z.B. den eindrucksvollen Bericht von Zander-Schneider (2011).

48 Füsgen (1995), 25.

49 Vgl. dazu auch Schmidhuber et al. (2014), 99-132.

50 Das lässt sich beispielsweise auch im Supermarkt oder in der Bahn feststellen, wenn ältere Menschen länger brauchen, um zu bezahlen oder ihren Sitzplatz zu finden. In solchen Situationen machen sich schnell Ungeduld und Unverständnis bei anderen bemerkbar.

51 Vgl. Gronemeyer (2013), 211.

52 Vgl. zur Lebensqualität bei Demenz Schmidhuber (2015). 
Gronemeyer ist keineswegs daran gelegen, die Erkrankung zu bagatellisieren, vielmehr appelliert er daran, Menschen mit Demenz »anders wahrzunehmen: Sie gehören als Bürgerinnen und Bürger zu uns, und es ist unsere Aufgabe, sie so gut wie möglich zu umsorgen, sie zu respektieren und sie, wenn möglich, zu Wort kommen zu lassen. « ${ }^{53}$

Es geht also darum, Menschen mit Demenz nicht aufgrund ihrer Erkrankung zu diskriminieren und abzuschieben. Die Diagnose Demenz verleitet dazu, die Betroffenen aufgrund der Symptome nicht mehr ernst zu nehmen, deshalb soll in der Sicht Gronemeyers nicht die Demenz im Mittelpunkt stehen, sondern der Mensch, der als soziales Wesen der Fürsorge und Zuwendung anderer bedarf. Gronemeyers Weg, Demenz nicht als Krankheit zu bezeichnen, zielt genau darauf ab.

Wenn es nun aber darum geht, das Menschenrecht auf ärztliche Versorgung und soziale Leistungen für ein möglichst gutes Leben zu gewährleisten, kann es besser sein, Demenz objektiv als Krankheit wahrzunehmen. Denn bei einer Erkrankung werden mehr und andere Versorgungsstrukturen benötigt als bei Gesundheit. Das muss jedoch Gronemeyers Forderung nach Integration der Betroffenen in die Gesellschaft nicht ausschließen. Besonders bei Demenz variiert das Leiden der Betroffenen sehr nach Stadium und Tagesverfassung. Deshalb ist es hier hilfreicher, Demenz von vornherein als Krankheit anzuerkennen und nicht nur an dem subjektiven Leid festzumachen.

\section{SCHLUSSÜBERLEGUNGEN}

In diesem Beitrag wurde versucht zu klären, welche Bedeutung den Menschenrechten bei subjektivem Leid und Krankheitsempfinden zukommt. Es scheint auf der Hand zu liegen, dass Menschenrechte nicht bei jedem individuellen Leid greifen können. Denn Leiden ist Teil der conditio humana. ${ }^{54}$ Auch Liebeskummer, Trauer um den Tod eines geliebten Menschen und ähnliche Lebenssituationen rufen Leid hervor. Jeder Mensch leidet anders, mehr oder weniger lange und intensiv, dennoch würden wir nicht die Menschenrechte für die Behebung solcher Leiderfahrungen verantwortlich

53 Gronemeyer (2013), 255.

54 Vgl. dazu auch den Beitrag von Bielefeldt in diesem Band. 
machen. Das Menschenrecht auf ein erreichbares Höchstmaß an körperlicher und geistiger Gesundheit kann nicht bedeuten, dass Menschen einen Anspruch auf ein leidfreies Leben haben. Vielmehr geht es um einen diskriminierungsfreien Zugang zu einem Lebensstandard, der Gesundheit unterstützt. Nahrung, Wohnung, Kleidung, ärztliche Versorgung und soziale Absicherung im Falle von widrigen Lebensumständen sind Grundlagen, die die Menschenrechte gewährleisten sollen. Auf dieser Basis kann dann mit subjektivem Leid angemessen umgegangen werden. Das ist es, was die Menschenrechte auch in diesem Kontext leisten sollen.

Es geht aber umgekehrt keineswegs darum, Menschen, die sich aufgrund eines subjektiven Leids krank fühlen, nicht ernst zu nehmen, auch wenn nicht alles mit einem menschenrechtlichen Anspruch verbunden werden kann. So erfordert der Umgang mit subjektivem Leiden an Kinderlosigkeit und Alter Coping-Strategien, die eine Gesellschaft in Form von z.B. Therapien bereitstellen kann. Oder wenn Menschen in Beratungsstellen bewusst gemacht wird, dass die Adoption von Kindern auch eine Option sein kann oder dass das Alter und seine Veränderungen ein wichtiger Lebensabschnitt und Zeichen der Reife sind, kann vielleicht so manches Leid, wenn nicht behoben, so doch gemildert werden. Die gesellschaftlich weit verbreiteten Erwartungshaltungen an Individuen gilt es zu hinterfragen und kritisch zu betrachten. Der Einfluss des Intersubjektiven ist nicht zu unterschätzen und es braucht oftmals viel Mut und Kraft, den in der Gesellschaft aufgestellten Erwartungen bewusst nicht zu entsprechen, nicht mit dem Strom zu schwimmen, sondern eigene, individuelle Wege des Umgangs mit z.B. ungewollter Kinderlosigkeit oder Altern zu finden. Und es bedarf des Bewusstseins, dass sich das Verständnis von Gesundheit und Krankheit aufgrund gesellschaftlicher Veränderungen stets wandelt und gerade deshalb ein individueller Weg im Umgang mit bestimmten Phänomenen gefunden werden sollte.

Bei Alzheimer-Demenz geht es allerdings darum, Menschen mit dieser Erkrankung Inklusion in die Gesellschaft und Mitspracherecht bei ihren Behandlungen zu ermöglichen. ${ }^{55}$ Selbst wenn Menschen an ihrer Demenz

55 Vgl. dazu auch Grover (2011), v.a. die Empfehlung auf S. 19: »Developing and implementing mechanisms to protect the rights of older persons if/when they are deemed incapable of providing informed consent to any treatment and/or other 
aufgrund des Vergessens nicht (mehr) leiden, ist es wichtig, ihnen einen Lebensstandard zu gewähren, der ihnen bestmögliche Versorgung zusichert. Denn Menschen mit kognitiven Beeinträchtigungen zu schützen und in ihrem Lebensalltag zu unterstützen, trifft wiederum genau das, was mit Art. 25.1 der Allgemeinen Erklärung der Menschenrechte intendiert wird.

\section{LITERATUR}

Ach, Johann S./Lüttenberg, Beate/Quante, Michael (Hg.) (2014): wissen leben - ethik. Themen und Positionen der Bioethik, Münster: Mentis.

Alzheimer's Disease International (2013): World Alzheimer Report 2013, Journey of Caring. An analysis of long-term care for dementia, London, Online: www.alz.co.uk/research/world-report-2013 [09.11.2015].

Anselm, Rainer (2013): »Kinderlosigkeit als Krankheit. Anthropologische und ethische Aspekte«, in: Maio et al. (2013), 96-113.

Birnbacher, Dieter (2014): »Medikalisierung und Krankheitsbegriff«, in: Ach et al. (2014), 203-211.

Beck-Gernsheim, Elisabeth (2013): »Kinderwunsch ohne Grenzen? Globalisierte Fortpflanzungsmedizin und neue Formen der Elternschaft«, in: Maio et al. (2013), 337-354.

Boorse, Christopher (1977): »Health as a theoretical concept«, in: Philosophy of Science 44 (1997), 542-573.

Bozzaro, Claudia (2012): »Der Traum ewiger Jugend. Anti-Aging-Medizin als Verdrängungsstrategie eines Leidens an der verrinnenden Zeit«, in: Maio (2012a), 219-248.

Bozzaro, Claudia (2014): Das Leiden an der verrinnenden Zeit. Alter, Leid und Zeit am Beispiel der Anti-Aging-Medizin, Stuttgart: frommannholzboog.

Breitbach, Elmar (2013): Künstliche Befruchtung: Welche Krankenkassen zahlen mehr? Online: www.wunschkinder.net/aktuell/gesellschaft/kranken kassen/kunstliche-befruchtung-welche-krankenkassen-zahlen-mehr-5265/ [21.10.2015].

medical intervention due to injury, disease or chronic conditions such as dementia.« 
Bröckling, Ulrich/Krasmann, Susanne/Lemke, Thomas (Hg.) (2000): Gouvernementalität der Gegenwart. Studien zur Ökonomisierung des Sozialen, Frankfurt/M.: Suhrkamp.

Daniels, Norman (2008): Just health. Meeting health needs fairly, New York: Cambridge University Press.

Eichinger, Tobias (2012): »Anti-Aging als Medizin? Altersvermeidung zwischen Therapie, Prävention und Wunscherfüllung«, in: Maio (2012a), 118-155.

Eichinger, Tobias (2013): Jenseits der Therapie. Philosophie und Ethik wunscherfüllender Medizin, Bielefeld: transcript.

Eissa, Tina-Louise (2014): Gesünder, intelligenter, perfekt? Selbstgestaltung durch Enhancement im Kontext pluralistischer Ethik, Freiburg: Alber.

Frewer, Andreas/Reis, Andreas/Bergemann, Lutz (Hg.) (2014): Gute oder vergütete Behandlung? Ethische Fragen der Gesundheitsökonomie, Jahrbuch Ethik in der Klinik, Bd. 7, Würzburg: Königshausen \& Neumann.

Friedrich, Daniel R. (2014): Solidarische Gesundheitsversorgung. Ein deliberativer Ansatz ihrer Rechtfertigung, Leipzig: Dissertation Universitätsbibliothek Leipzig.

Füsgen, Ingo (1995): Demenz. Praktischer Umgang mit der Hirnleistungsstörung, München: MMV.

Grey, Aubrey de (2005): „Life extension, human rights, and the rational refinement of repugnance«, in: Journal of Medical Ethics 31 (2005), 659-663.

Gronemeyer, Reimer (2013): Das 4. Lebensalter. Demenz ist keine Krankheit, München: Pattloch.

Grover, Anand (2011): Thematic study on the realization of the right to health of older persons by the Special Rapporteur on the right of everyone to the enjoyment of the highest attainable standard of physical and mental health, Geneva, 4 July 2011, A/HRC/18/37.

Heil, Reinhard (2010): »Trans- und Posthumanismus. Eine Begriffsbestimmung«, in: Hilt et al. (2010), 127-149.

Hilt, Annette/Jordan, Isabella/Frewer, Andreas (Hg.) (2010): Endlichkeit, Medizin und Unsterblichkeit. Geschichte - Theorie - Ethik, Stuttgart: Steiner. 
Illich, Ivan (1975): »The medicalization of life«, in: Journal of Medical Ethics 1, 2 (1975): 73-77.

Knell, Sebastian/Weber, Marcel (Hg.) (2009): Länger leben? Philosophische und biowissenschaftliche Perspektiven, Frankfurt/M.: Suhrkamp.

Kovács, Laszlo/Kipke, Roland/Lutz, Ralf (Hg.) (2015): Lebensqualität in der Medizin. Messung, Konzepte, Konsequenzen, Berlin: Springer.

Krämmer, Johannes/Schmidhuber, Martina (2015): »Ewige Jugend und ewiges Leben als erstrebenswerte Ziele? Antworten aus der Philosophie auf die Anti-Aging-Debatte und auf die trans- und posthumanistischen Unsterblichkeitsvisionen «, in: Aufklärung \& Kritik 1 (2015), 29-48.

Kruse, Andreas/Wahl, Hans-Werner (2010): Zukunft Altern. Individuelle und gesellschaftliche Weichenstellungen, Heidelberg: Spektrum Akademischer Verlag.

Lacina, Katharina (2009): Tod, Wien: UTB Profile.

Lemke, Thomas (2000): »Die Regierung der Risiken. Von der Eugenik zur genetischen Gouvernementalität«, in: Bröckling et al. (2000), 227-264.

Maier, Wolfgang/Schulz, Jörg B./Weggen, Sascha/Wolf, Stefanie (2011): Alzheimer \& Demenzen verstehen. Diagnose, Behandlung, Alltag, Betreuung, Stuttgart: Trias.

Maio, Giovanni (Hg.) (2012a): Altwerden ohne alt zu sein?, Freiburg: Alber.

Maio, Giovanni (2012b): „Vom Sinn des Alters. Reflexionen zum Alter jenseits des Fitnessimperativs «, in: Maio (2012a), 11-19.

Maio, Giovanni (2013): »Wenn die Technik die Vorstellung bestellbarer Kinder weckt«, in: Maio et al. (2013), 11-37.

Maio, Giovanni/Eichinger, Tobias/Bozzaro, Claudia (Hg.) (2013): Kinderwunsch und Reproduktionsmedizin. Ethische Herausforderungen der technisierten Fortpflanzung, Freiburg: Alber.

Mayer-Olkin, Alexander (2015): Entgrenzungen zwischen Natur und Kultur. Gibt es eine gesellschaftliche Tendenz zur Medikalisierung? Das Beispiel »ADHS«, München: GRIN Verlag.

Nordenfelt, Lennart (1995): On the nature of health: An action-theoretic approach, Dordrecht: Springer.

Nordenfelt, Lennart (2012): »Der Gegensatz zwischen naturalistischen und holistischen Theorien von Gesundheit und Krankheit«, in: Rothhaar/ Frewer (2012), 89-104. 
Rothhaar, Markus/Frewer, Andreas (Hg.) (2012): Das Gesunde, das Kranke und die Medizinethik. Moralische Implikationen des Krankheitsbegriffs, Stuttgart: Steiner.

Schicktanz, Silke/Schweda, Mark (2012a): »Im Spannungsfeld von ProAge und Anti-Aging: Interdisziplinäre Diskurse über das Altern und die Rolle der Medizin«, in: Schicktanz/Schweda (2012b), 9-19.

Schicktanz, Silke/Schweda, Mark (Hg.) (2012b): Pro-Age oder Anti-Aging? Altern im Fokus der modernen Medizin, Frankfurt/M.: Campus.

Schmidhuber, Martina/Bergemann, Lutz/Legal, Friederike (2014): »Sinnvolle Vergütung für gute Behandlung? Zum Zusammenhang ökonomischer und ethischer Überlegungen am Beispiel der frühen Demenzdiagnose«, in: Frewer et al. (2014), 99-132.

Schmidhuber, Martina (2015): »Alzheimer-Demenz und Lebensqualität Ein Widerspruch? Ein narrativer Zugang zur Lebensqualität von Menschen mit Demenz«, in: Kovács et al. (2015), 273-286.

Schöne-Seifert, Bettina (2007): Grundlagen der Medizinethik, Stuttgart: Alfred Kröner.

Schramme, Thomas (2000): Patienten und Personen. Zum Begriff der psychischen Krankheit, Frankfurt/M.: Fischer.

Schramme, Thomas (2009): Ist Altern eine Krankheit?, in: Knell/Weber (2009), 235-263.

Schramme, Thomas (Hg.) (2012a): Krankheitstheorien, Berlin: Suhrkamp.

Schramme, Thomas (2012b): »Einleitung: Die Begriffe `Gesundheit` und >Krankheit` in der philosophischen Diskussion«, in: Schramme (2012a), 9-37.

Schweda, Mark/Marckmann, Georg (2012): »Zwischen Krankheitsbehandlung und Wunscherfüllung: Anti-Aging-Medizin und der Leistungsumfang solidarisch zu tragender Gesundheitsversorgung «, in: Ethik in der Medizin 24 (2012), 179-191.

Steinfath, Holmer (2001): Orientierung am Guten. Praktisches Überlegen und die Konstitution von Personen, Frankfurt/M.: Suhrkamp.

Wardrope, Alisdair (2015): »Medicalization and epistemic injustice«, in: Medical Health Care and Philosophy 18 (2015), 341-352.

Zander-Schneider, Gabriela (2011): Sind Sie meine Tochter? Leben mit meiner alzheimerkranken Mutter, Reinbek bei Hamburg: rororo. 



\title{
Brauchen wir ein Recht auf Krankheit?
}

\author{
Historische und theoretische Überlegungen \\ im Anschluss an Juli Zehs Roman Corpus Delicti
}

CAROLINE WELSH

\section{EINLEITUNG}

»Gesundheit ist ein Zustand des vollkommenen körperlichen, geistigen und sozialen Wohlbefindens - und nicht die bloße Abwesenheit von Krankheit. [...] Gesundheit ist das Ziel des natürlichen Lebenswillens und deshalb natürliches Ziel von Gesellschaft, Recht und Politik. Ein Mensch, der nicht nach Gesundheit strebt, wird nicht krank, sondern ist es schon. ${ }^{1}$

Mit dem ersten Satz beginnt, mit den letzten Sätzen endet das Zitat aus dem Vorwort Heinrich Kramers zu seinem Buch Gesundheit als Prinzip staatlicher Legitimation, mit dem Juli Zeh ihren Roman Corpus Delicti. Ein Prozess (2009) einleitet. Heinrich Kramer ist selbst eine Figur des Romans und wirkmächtigster Vertreter sowie rücksichtsloser Verfechter einer Gesundheitspolitik, METHODE genannt, die im Namen der Krankheitsprävention in alle Lebensbereiche der Bevölkerung eingreift. Das Zitat entstammt also einem fiktiven Buch geschrieben von einer fiktiven Figur aus der Welt eines Romans. Es zitiert zugleich im einleitenden Satz fast wörtlich die Gesundheitsdefinition der Weltgesundheitsorganisation (WHO).

1 Zeh (2010), 7-8. 
In der Welt des Romans ist um die Mitte des 21. Jahrhunderts, also in nicht allzu weiter Ferne, die Zukunftsvision einer krankheitsfreien Gesellschaft mit Hilfe einer vom Staat gesteuerten, auf biomedizinischen Erkenntnissen, Informationstechnologien und medizintechnischen Überwachungssystemen beruhenden, umfassenden Gesundheitspolitik Realität geworden. Der Roman handelt vom Preis, den der Einzelne und die Gesellschaft für diese »heile Welt« zu zahlen haben. Gesundheitsschädliches Verhalten, wie der Genuss »toxischer Substanzen« (Kaffee, Alkohol, Nikotin), aber auch mangelnde Bewegung am vernetzten Hometrainer, sind strafbar, die Wahl eines immunologisch nicht geeigneten Partners ein Kapitalverbrechen. Um vor Gericht geladen zu werden, reicht die Vernachlässigung der regelmäßigen Meldepflicht gesundheitsrelevanter Daten wie Schlafbericht, Ernährungsbericht, Blutdruckmessung und Urintest aus. Ohnehin sind sämtliche Vitalparameter einschließlich einer vollständigen elektronischen Patientenakte auf einem in den Oberarm eingepflanzten Chip gespeichert und vor Gericht ebenso wie vom Arzt jederzeit abrufbar. ${ }^{2}$

Die Menschenrechte des Einzelnen, insbesondere das Recht auf den Schutz der Privatsphäre (u.a. Datenschutz), das Selbstbestimmungsrecht über den eigenen Körper (u.a. körperliche Unversehrtheit), das Recht auf freie Gestaltung der persönlichen Lebensführung (u.a. freie Partnerwahl), wie sie allesamt in Art. 8 der Europäischen Menschenrechtskonvention (EMRK) zum »Recht auf Achtung des Privatlebens« niedergelegt sind, werden durch diese Maßnahmen im Namen der Gesundheit massiv eingeschränkt. Die Rechtfertigung solcher Beschränkungen leitet sich aus dem zentralen Grundsatz der METHODE her, dass das persönliche Interesse und das des Systems in dem Bestreben, Krankheit zu vermeiden, zur Deckung kommen. ${ }^{3}$ Daher ist auch psychischer Schmerz, weil er die Krankheitsanfälligkeit erhöht, keine Privatangelegenheit, sondern ein umgehend durch Betreuungs- und Rehabilitationsmaßnahmen zu behebender Zustand. »Es

2 Zur Aktualität dieser informationstechnologischen Visionen vgl. Gerhard Kunnerts (2011) Ausführungen zur elektronischen Patientenakte, zu DNA-Chips für die schnelle Genomanalyse sowie implementierten RFID-Chips zum »Auslesen von Vitalparametern (Blutdruck, Blutwerte, etc.)« (ebd., 134). 2015 hat der Deutsche Ethikrat Die Vermessung des Menschen - Big Data und Gesundheit zum Thema seiner Jahrestagung gemacht, vgl. Deutscher Ethikrat (2015).

3 Zeh (2010), u.a. 36-43, 87, 160-161, 180. 
besteht«, so warnt die Richterin mit dem sprechenden Namen »Sophie« die wegen ihres toten Bruders aus dem Gleichgewicht geratene Protagonistin, »eine enge Verbindung zwischen dem persönlichen und dem allgemeinen Wohl, die in solchen Fällen keinen Raum für Privatangelegenheiten lässt «. ${ }^{4}$ In einem sich derart über Gesundheit und Krankheitsprävention legitimierenden Staat ist die Einforderung eines Rechts auf Krankheit, wie Heinrich Kramer in einem mit »Recht auf Krankheit « überschriebenen Kapitel des Romans ausführt, ein »kriegerischer Angriff, dem wir mit Krieg begegnen werden. ${ }^{5}$ Die R.A.K. (das Akronym steht, mit Anspielung auf die R.A.F., für das Recht auf Krankheit), eine tatsächliche oder vom System erfundene Widerstandsbewegung, gilt entsprechend als methodenfeindliches, ja terroristisches Netzwerk.

Die literaturwissenschaftliche Forschung hat Zehs biomedizinische Dystopie als »literarische Ausarbeitung einer zukünftigen Biopolitik im Sinne Foucaults « ${ }^{6}$, als eine »auf Gesundheit gründende, biopolitisch ausgerichtete Herrschaft über das Leben ${ }^{7}$ auf der Basis biomedizinischen Wissens, realisiert durch eine »Diktatur der Hygiene ${ }^{8}$ gelesen. Dass sich die Überwachungstechniken hier auf eine vollständige Kontrolle nicht des Denkens, sondern des Körpers beziehen, unterscheide Zehs Roman von den klassischen politischen Dystopien. ${ }^{9}$ Mit der »Terrorherrschaft biologische[r] Vernunft « ${ }^{10}$ knüpfe Corpus Delicti zudem an die Analysen Adornos und Horkheimers zum Umschlag instrumenteller Vernunft in Barbarei in der Dialektik der Aufklärung ebenso an wie an Ulrich Becks Analyse der Risikogesellschaft. ${ }^{11}$

Im Folgenden soll, der Anspielung des Romans auf die Gesundheitsdefinition der WHO folgend, zunächst nach einigen konkreten Anknüpfungspunkten dieser aus den gegenwärtigen Gegebenheiten und Tendenzen heraus entwickelten Zukunftsdystopie gefragt werden. Der Roman wird dabei

4 Zeh (2010), 51-54 und 57-59, 58. Zitat 58.

5 Ebd., 89.

6 Geisenhanslüke (2013), 232.

7 Hilmes (2013), 71.

8 Geisenhanslüke (2013), 228.

9 Vgl. ebd.

10 Ebd., 232.

11 Vgl. ebd., 223-227. 
als Problemanzeige und Warnung vor einer möglichen fatalen Dynamik gesundheitspolitischer Entwicklungen auf der Grundlage biomedizinischen Wissens, medizintechnischer Überwachungsmöglichkeiten und gesundheitsökonomischer Zwänge verstanden. ${ }^{12}$ Er stellt die Gefahr eines Missbrauchs des Menschenrechts auf Gesundheit in Kombination mit gesundheitspolitischen Maßnahmen in Aussicht. Inwieweit dieses »Recht auf Gesundheit« in seinem Zusammenspiel mit den anderen Menschenrechten vor einem solchen Missbrauch schützen kann, soll dann in einem zweiten Schritt geklärt werden. Gibt es aus menschenrechtlicher Sicht konzeptionelle und rechtlich verankerte Sicherungen, die einen solchen Missbrauch des »Rechts auf Gesundheit« verhindern - oder muss aus der Möglichkeit des Missbrauchs gefolgert werden, dass wir zusätzlich ein menschenrechtlich abgesichertes »Recht auf Krankheit«, zumindest auf Krank-Sein ohne Diskriminierung und Stigmatisierung brauchen?

\section{VON DER ॥UMFASSENDEN《 ZUR „VOLLKOMMENEN« GESUNDHEIT}

Die WHO erklärt in ihrer Präambel zur Deklaration von 1946 den »Besitz des bestmöglichen Gesundheitszustandes « als ein allen Menschen ohne Ausnahme zustehendes »Grundrecht« und definiert Gesundheit als »Zustand des vollständigen körperlichen, geistigen und sozialen Wohlergehens und nicht nur das Fehlen von Krankheit und Gebrechen «. ${ }^{13}$ Gesundheit wird hier als ein, die gesamte Lebenswelt, also auch die Umwelt und das soziale Umfeld ${ }^{14}$, einbeziehendes »Wohlergehen« gefasst. Diese umfas-

12 Vgl. Zehs Äußerung in einem österreichischen Fernsehinterview: »Eigentlich sehe ich das ausschließlich als Warnung an die jetzige Gesellschaft. Ich habe die Zukunftsform nur benutzt, um ein bisschen überspitzen und übertreiben zu dürfen. Ansonsten ist die im Roman vorherrschende Mentalität eine, die wir jetzt schon sehen können. « Brodnig (2009), zitiert aus Maierhofer (2012), 32.

13 Im Original: »Health is a state of complete physical, mental and social wellbeing and not merely the absence of disease or infirmity." World Health Organization (2009), 100.

14 Vgl. ebd., »Umgebungshygiene« (Art. 2.i) und der »geistigen Hygiene« (Art. 2.m). 
sende Gesundheitsdefinition bleibt auch für die späteren WHO-Dokumente charakteristisch. Sie geht in die Erklärung von Alma-Ata (1978) zur »primären Gesundheitsversorgung « insbesondere in den Entwicklungsländern als "grundlegendes Menschenrecht ${ }^{15}{ }^{15}$ ebenso ein wie in die sich stärker »mit Erfordernissen in den Industrieländern « ${ }^{16}$ befassende Ottawa-Charta zur Gesundheitsförderung (1986). Die Erklärung von Alma-Ata fordert u.a.: "Aufklärung in Bezug auf vorherrschende Gesundheitsprobleme und die Methoden zu ihrer Verhütung und Bekämpfung«; Förderung der Nahrungsmittel- und Trinkwasserversorgung sowie »Impfung gegen die schwersten Infektionskrankheiten; Prävention und Bekämpfung endemischer Krankheiten; angemessene Behandlung der häufigsten Krankheiten und Verletzungen; und Versorgung mit unentbehrlichen Arzneimitteln «. ${ }^{17}$ Ziel der Ottawa-Charta ist demgegenüber eine grundlegende Neuorientierung der Gesundheitsdienste, welche die »Gesundheitsförderung « priorisiert. Zusammen mit dem Staat sollen die Gesundheitsdienste und -einrichtungen »ein Versorgungssystem [...] entwickeln, das auf die stärkere Förderung von Gesundheit ausgerichtet ist und weit über die medizinisch-kurativen Betreuungsleistungen hinausgeht. " ${ }^{18}$ Dazu gehören die »Schaffung gesundheitsförderlicher Lebenswelten«, beispielsweise durch die gesundheitsbewusste Gestaltung der Arbeits- und Freizeitbedingungen, sowie die gesundheitsbezogene Bildung der Bürger.

Während in der Ottawa-Charta ausschließlich der Begriff der »Gesundheitsförderung « verwendet wird, findet sich in jüngeren Ausführungen zur Neuausrichtung der Gesundheitssysteme eine Verschiebung hin zur »Krankheitsprävention«:

»Alle Länder müssen sich an geänderte demografische und epidemiologische Muster anpassen, insbesondere in Bezug auf Herausforderungen im Bereich der psychischen Gesundheit sowie chronischer und altersbedingter Erkrankungen. Dies erfordert eine Neuausrichtung der Gesundheitssysteme, so dass sie Krankheitsprävention priorisieren $[\ldots] \ll .{ }^{19}$

15 World Health Organization (1978), 1.

16 World Health Organization (1986), 1.

17 World Health Organization (1978), 2 (VII, 3).

18 World Health Organization (1986), 4 (»Gesundheitsdienste neu orientieren«).

19 World Health Organization (2013a), 10. 
Unterstützt vor allem durch die UN-Generalversammlung zur Prävention und Kontrolle nichtübertragbarer Krankheiten (2011), liegt der Fokus nun auf den NCDs (non-communicable diseases), insbesondere auf Herzkreislauferkrankungen, chronischen Lungenerkrankungen, Krebs und Diabetes. Ein Hauptinteresse gilt der Minimierung von Risikofaktoren, wobei ersichtlich wird, dass Krankheit auch als politisches Problem wahrgenommen wird. ${ }^{20}$ In den WHO-Erklärungen geraten im Zuge dieser Neuausrichtung Lebensstile mit höherem Krankheitsrisiko zunehmend in den Fokus. So in den Erklärungen von Aschgabat, Über die Prävention und Bekämpfung nichtübertragbarer Krankheiten (2013) und Wien, Über Ernährung und nicht übertragbare Krankheiten (2013), sowie in der Europäischen Charta zur Bekämpfung der Adipositas (2006) und in der Stockholmer Erklärung über Jugend und Alkohol (2001).

Der wünschenswerte Erfolg solcher präventivmedizinischer Programme soll hier keineswegs in Frage gestellt werden. Doch sollte zugleich dafür Sorge getragen werden, dass der Einzelne menschenrechtlich vor diskriminierenden Aspekten einer auf Risikominimierung hin ausgerichteten Krankheitsprävention geschützt ist. Sie ergeben sich aus der normativen Stoßrichtung der Argumentation, die eine moralische Wertung (oder über Krankenkassen eine ökonomische Benachteiligung) von abweichendem, nicht gesundheitsbewusstem Verhalten nahelegt und damit Gefahr läuft, eine Stigmatisierung der Betroffenen zu fördern.

Das »langfristige, durch die Methoden der >molekularen Medizin « erreichbare Ziel einer Welt ohne Krankheiten und eines mit den Methoden

20 Vgl. die Formulierung in der Erklärung von Aschgabat, World Health Organization (2013b), 1: „Wir nehmen die soliden wissenschaftlichen Belege dafür zur Kenntnis, dass die häufigsten nichtübertragbaren Krankheiten viel Leiden verursachen und die gesellschaftliche und wirtschaftliche Entwicklung in unseren Ländern untergraben. Diese Krankheiten sind mit vier weit verbreiteten Risikofaktoren - Tabakkonsum, schädlicher Alkoholkonsum, ungesunde Ernährung und Bewegungsmangel - verknüpft und können bis in späte Lebensphasen weitgehend vermieden werden.« Zur bisher wenig aufgearbeiteten Geschichte der Gesundheitsprävention vgl. Lengwiler/Madarász (2010), zur Soziologie und Ratio der Prävention u.a. mit Beispielen aus dem Gesundheitswesen: Bröckling (2008). 
der Molekulargenetik vollständig modifizierbaren Menschen « ${ }^{21}$ steht unhinterfragt auch im Hintergrund aktueller biomedizinischer Forschung. Die von den Ergebnissen der Molekulargenetik und Biomedizin gestützte Fokussierung auf die Krankheitsprävention läuft zudem Gefahr, biologisch definierte Gesundheit zu einem »zentralen gesellschaftlichen Gut und einem das gesamte Verhalten normierenden Wert « ${ }^{22}$ zu erheben. Grundsätzlicher noch gälte es daher zu fragen, wie sich eine solch starke Konzentration auf »Gesundheit«, verbunden mit den Visionen einer biomedizinischen Optimierbarkeit des Menschen, auf die Wahrnehmung Kranker, auf das Menschenbild insgesamt und auf unsere Vorstellung, wie wir leben und sterben wollen, auswirkt. Führt die biomedizinische Verheißung einer zukünftigen Welt ohne Krankheiten zu einer »substantiellen Verschiebung in der Bewertung des Menschen, und zwar weg vom unbedingten Respekt vor der Integrität hin zur prinzipiellen Zustimmung zu Verfügbarkeit, Manipulierbarkeit und auch Verwertbarkeit menschlichen Lebens «? ${ }^{23}$

Laufen wir Gefahr, mit dem Glauben an eine grundsätzliche Vermeidbarkeit von Krankheit bis ins hohe Alter hinein nicht nur die Krankheit, sondern auch das Sterben aus unserem Leben zu verdrängen? Canguilhem versteht Krankheiten als »Werkzeuge des Lebens, durch die sich der Lebende, wenn es ein Mensch ist, gezwungen sieht, seine Sterblichkeit einzugestehen «. ${ }^{24}$ Nach Zygmunt Bauman ist in der Moderne die Sterblichkeit als etwas allem Lebenden Zugehöriges »in verschiedene Ereignisse des privaten Todes dekonstruiert worden, die alle eine vermeidbare Ursache haben $« .{ }^{25}$ Dadurch sei an die Stelle des Wissens um die eigene Sterblichkeit die kontinuierliche Sorge um die Gesundheit und die Überzeugung von der prinzipiellen Heilbarkeit aller Krankheiten getreten.

Die Vorstellung, dass ein gesundes, aktives Leben bis ins hohe Alter durch eine Kombination moderner Medizin, gesunder Umwelt und gesundheitsbewusstem Verhalten des Einzelnen möglich sei, scheint längst in der Mitte der Gesellschaft angekommen. In extremer Form findet der gesundheitspolitische Diskurs der WHO zur Krankheitsprävention durch die ge-

21 Roelcke (2003), 122.

22 Roelcke (2003), 122.

23 Ebd., 123.

24 Canguilhem (2013a), 42.

25 Bauman (1994), 207. 
zielte Einflussnahme auf das Gesundheitsbewusstsein des Einzelnen sein gesellschaftliches Pendant in einer neue Fitnesskultur, deren Ziel die Optimierung der individuellen Gesundheit auf der Basis medizinischen Wissens und medizinischer Technologien ist. ${ }^{26}$ Im Rahmen dieser freiwilligen Medikalisierung des Fitnessbereichs erfreut sich der Markt für digitale Gesundheit inzwischen - über die Quantified-Self-Communitiy (Selbstvermessung des Ichs) hinaus - einer stetig anwachsenden Zahl gewissenhafter Überwacher der eigenen Gesundheit. Mit Hilfe kleiner, am Körper tragbarer Messgeräte werden regelmäßig, bei unterschiedlicher körperlicher Belastung zunehmend mehr Vitalwerte (Blutdruck, Pulsfrequenz, Atmung, Blutzuckerspiegel), aber auch »Daten zu Ernährung, Gesundheit, Körperumfang oder Verhalten ${ }^{27}$ gemessen, vernetzt und dann am Heimcomputer ausgewertet. Hier scheint die »Optimierung biologisch definierter Körperzustände« mit Hilfe der Selbstvermessung (Selftracking) auf der Basis einer freiwilligen Selbstdisziplinierung und -kontrolle bereits zum obersten Wert individuellen Verhaltens geworden zu sein. Handelt es sich hier um eine autonome Entscheidung des Einzelnen zur Risikominimierung und Krankheitsprävention oder um die Internalisierung von Verhaltensnormen, die sich mit Foucault als Ersetzung der »traditionellen Mechanismen des Überwachens und Strafens [...] durch ein Regime freiwilliger Selbstkontrolle ${ }^{28}$ beschreiben lassen? Wie auch immer man diese neue, durch medizinische Überwachungstechnologien ermöglichte Sorge um sich einordnen möchte, bleiben doch die Rückkopplungseffekte der medikalisierten Fitnesskultur an das Gesundheitssystem bedenklich. Denn zunehmend interessieren sich inzwischen auch Krankenkassen für diese von ihren Mitgliedern selbst generierten Körperdaten. ${ }^{29}$ Einige locken bereits mit Bonuszahlungen für die Teilnahme an digitalen, personalisierten Präventionsprogrammen (AOK mobil vital; FitCheck-App der DAK). Damit wird die freiwillige Selbstkontrolle langfristig durch eine Überwachung von oben ergänzt, und es könn-

26 Vgl. Krüger-Brand (2014), an deren Darstellung ich mich hier stark anlehne, sowie die Jahrestagung des Deutschen Ethikrats (2015), dort insbesondere das Audioprotokoll des Vortrags von Dabrock (2015).

27 Krüger-Brand (2014), 1.

28 Bröckling (2004), 214; zum Wechselverhältnis zwischen autonomer und heteronomer Selbstdisziplinierung vgl. Wolff (2010), 193-196.

29 Vgl. Krüger-Brand (2014), 4-7. 
ten ökonomische Anreize zur Verhaltensänderung sukzessive in eine erzwingbare Pflicht zur Gesundheit umschlagen.

Zehs Bezug auf die Gesundheitsdefinition der WHO als Ausgangspunkt ihrer Dystopie gewinnt vor dem Hintergrund dieser Kombination aus gesundheitspolitischen Präventionsprogrammen, molekulargenetischen $\mathrm{Zu}$ kunftsträumen und privaten Selbstdisziplinierungsmaßnahmen an kritischer Tiefenschärfe. Die dem Einzelnen im Roman als »Pflicht zur Gesundheit« im Sinne eines gesunden Lebensstils auferlegten und zugleich von einem Großteil der Bevölkerung freiwillig durchgeführten Hygienemaßnahmen und Fitnessprogramme zur Erhaltung einer »vollkommenen Gesundheit« werden als fiktionale Überspitzung aktueller gesundheitspolitischer Diskurse sowie dazugehöriger Praktiken des Anreizes zu gesundheitsförderlichem Verhalten kenntlich.

Mit Hilfe einer leichten, aber folgenreichen Verschiebung in der Wortwahl von einem »bestmöglichen« und »umfassenden« (engl. complete) »körperlichen, geistigen und sozialen Wohlbefinden « (Ottawa-Charta) hin zu einem »vollkommenen« (engl. absolute) »körperlichen, geistigen und sozialen Wohlbefinden « (Corpus Delicti) verlagert der Roman das Gewicht der Gesundheitsdefinition grundlegend. An die Stelle einer Fokussierung sowohl medizinischer als auch ökologischer und sozialökonomischer Aspekte (umfassend) in der WHO-Definition tritt der Idealzustand einer vollkommenen Gesundheit für jedermann. Damit einher geht ein »methodenrechtlicher Anspruch auf Gesundheit « ${ }^{30}$, der das »Recht auf Gesundheit« zu einem vom Staat zu gewährleistenden Schutz vor Krankheit, und damit zu einem Rechtsanspruch auf Gesundsein uminterpretiert. ${ }^{31}$ Die zur Gewährleistung dieses Rechts notwendigen Präventionsmaßnahmen legitimieren ihrerseits gesundheitspolitische Maßnahmen, die grundlegende menschenrechtliche Freiheiten außer Kraft setzen. Ein so verstandenes Recht auf Gesundheit hat mit dem menschenrechtlichen »Recht auf Gesundheit« wenig gemeinsam.

30 Zeh (2010), 85.

31 Vgl. ebd., 180. 


\section{GESUNDHEITSRECHT UND GESUNDHEITSPFLICHT}

Die konzeptionellen Vorläufer des heutigen Rechts auf Gesundheit finden sich in den spätaufklärerischen Schriften zur Medicinalpolizey von Johann Peter Frank (1779-1788), ${ }^{32}$ dem »bis dahin umfassendste[n] Versuch, das gesamte öffentliche und private Leben unter gesundheitlichen Gesichtspunkten regeln zu wollen «. ${ }^{33}$ Zeitgleich mit diesen sozialdisziplinierenden Maßnahmen spätabsolutistischer Polizeiwissenschaft wird die Sorge um die Gesundheit in der bürgerlichen Gesellschaft zum wichtigsten Element aufgeklärt-rationaler Lebensführung, mit dem sich das politisch machtlose Bildungsbürgertum vom Adel absetzt und zugleich weitere Gesellschaftsschichten in die bürgerliche Gesellschaft integriert. ${ }^{34}$ Zusammen mit der Vorstellung, dass »Krankheit durch vernünftige Verhaltensregeln und Vorschriften« vermieden werden könne, entwickelt sich ein bürgerlicher Normendiskurs, in dem Gesundheit und gesunde Lebensführung zur »Pflicht gegenüber der Allgemeinheit « werden. ${ }^{35}$ Wer dennoch krank wird, wird stigmatisiert: »Der Bürger muß gesund und arbeitsfähig seyn, um das Seine zur Erhaltung des Ganzen beitragen zu können. Ist er dies nicht, so fällt er dem Staate zur Last und ist schlimmer, als ein todtes Mitglied. « ${ }^{36}$

Mit der Herausbildung des europäischen Gesundheitswesens im 19. Jahrhundert gewinnt das Recht auf Gesundheit - nicht zuletzt aufgrund des durch Verstädterung und Industrialisierung zunehmend deutlicher werdenden Zusammenhangs zwischen Armut, Arbeitslosigkeit und erhöhter Krankheitsanfälligkeit sowie deren wirtschaftlichen und ökonomischen Folgen $^{37}$ - zunehmend an Kontur. Im deutschen Kontext ist hier vor allem die 1848/49er Medizinalreformbewegung wichtig. So versteht Rudolf Virchow, der Begründer der sozialen Medizin, das »Wohlsein aller Staats-

32 Vgl. Schulz (1987), 25-29.

33 Labisch (1992), 89.

34 Vgl. Frevert (1984), 28-36.

35 Zitate ebd., 33 und 31. Frevert geht hier auch kurz auf die Utopie einer krankheitsfreien Gesellschaft im bürgerlichen Gesundheitsdiskurs der Aufklärung u.a. im Kontext von Rousseaus Gesellschaftsvertrag ein.

36 Röber (1806), 15-16; zitiert aus Frevert (1984), 32.

37 Vgl. Frevert (1984), Labisch (1992), Hardy (2005). 
bürger « ${ }^{38}$ als Staatszweck und knüpft es aufgrund der engen Verbindung zwischen Armut und Krankheit an die Forderung eines »Recht[s] auf Arbeit«. ${ }^{39}$ Der deutschsprachige »Klassiker des Rechts auf Gesundheit « ${ }^{40}$ entstammt der mehrbändigen Verwaltungslehre des Staatsrechtlers Lorenz von Stein. Dort behandelt er im dritten Band, "Das Gesundheitswesen«, selbiges als eine selbständige Wissenschaft innerhalb der Staatswissenschaft. ${ }^{41}$ Steins Ausführungen sind interessant, weil sie die Sorge des Gesundheitswesens um die öffentliche Gesundheit unter expliziter Abstraktion vom kranken Individuum in das Zentrum des Staatsinteresses stellen und dennoch zugleich auch die selbstbestimmte Verfügbarkeit des Einzelnen über seine Gesundheit sicherzustellen suchen. Von Stein begründet zunächst die Notwendigkeit eines eigenständigen Gesundheitswesens innerhalb der Staatsverwaltung mit der gegenseitigen Bedingtheit von individuellem, öffentlichem und auch staatlichem Wohl. Gesundheit kann nicht nur »vom Standpunkte des rein individuellen Zustandes gedacht« werden, weil »das Leben der Gesammtheit zur Bedingung der Gesundheit und Krankheit aller Einzelnen « wird. ${ }^{42}$ Letztere ist ihrerseits Voraussetzung eines lebendigen und gesunden Staatsorganismus ${ }^{43}$ : Als Bedingung für das Leben des Staates wird sie [die öffentliche Gesundheit] für ihn [den Staat] zu einem Gute; und er erkennt bald, daß dieses Gut nicht bloß für den Einzelnen, sondern auch für ihn selber einen hohen, ja einen unschätzbaren Werth habe. " ${ }^{44}$ Aufgabe des Gesundheitswesens ist es daher, die »öffentliche Gesundheit herzustellen ${ }^{45}$ und auch die medizinische Wissenschaft dahin zu bringen, »ihr ursprüngliches Gebiet des Einzellebens zu verlassen, und zuerst die Bedingungen der öffentlichen Gesundheit und ihre Gefahren im Gesammtleben zu suchen «. ${ }^{46}$

38 Virchow (1848/1983), 21-22; zitiert bei Schulz (1987), 33.

39 Schulz (1987), 32.

40 Ebd., 44.

41 Vgl. von Stein (1882).

42 Ebd., 20.

43 Zur Problematik der Organismus-Metaphorik in Staats- und Gesellschaftstheorien vgl. Lüdemann (2007).

44 Von Stein (1882), 22.

45 Ebd., 21.

46 Ebd., 22. 
Interessant ist nun, dass Stein in seiner Darstellung des Gesundheitswesens als Teilgebiet der inneren Verwaltung des »persönlichen Lebens « drei Prinzipien des Gesundheitswesens aufstellt, die sowohl inhaltlich als auch argumentativ zentrale Aspekte des menschenrechtlichen Rechts auf Gesundheit vorwegnehmen. So entspricht von Steins oberstes, leitendes Prinzip den Kernaussagen des späteren Rechts auf Gesundheit zu den staatlichen Schutz- und Gewährleistungspflichten: Aufgabe des Gesundheitswesens ist der Schutz des Einzelnen gegen die Gefährdungen seiner Gesundheit durch die Gemeinschaft (also durch Dritte oder die Umwelt) sowie die Förderung seiner Gesundheit durch die Bereitstellung einer medizinischen Versorgungsstruktur. Die anderen beiden Prinzipien schützen die Gesundheitsrechte des Einzelnen vor staatlichen Übergriffen und sichern seine universelle Geltung. Das »Prinzip der freien Persönlichkeit « ${ }^{47}$ legt fest, dass es »Sache der freien Selbstbestimmung des Einzelnen [ist], sich ihrer [der Angebote des Gesundheitswesens] zu bedienen oder nicht « ${ }^{48}$. Es schützt mit dem Verweis auf die »individuelle Freiheit« den Einzelnen vor einer Bevormundung durch das Gesundheitswesen. Das »sociale Verwaltungsprinzip « ${ }^{49}$ garantiert als drittes Prinzip, dass »dem Menschen an und für sich, unabhängig von seiner wirtschaftlichen und gesellschaftlichen Stellung « ${ }^{50}$ der Schutz und die Pflege seiner Gesundheit durch die Inanspruchnahme der »Leistungen der Verwaltung in Medicinal- und Sanitätswesen « ${ }^{51}$ ermöglicht wird.

Die Begründung dieses jedem Einzelnen qua seines Menschseins zukommenden Rechts auf Gesundheit ist eine ethische. Sie leitet sich aus der Anerkennung des »absoluten Werth[es]« der »Gesundheit auch des Einzelnen « als dem obersten »ethischen Princip« des Staates ab. ${ }^{52}$ Besonders wichtig ist diese Begründung, weil sie mit der Anerkennung des absoluten Werts der Gesundheit des Einzelnen ein deutliches Gegengewicht zu Steins früheren Ausführungen zur Bedeutung der öffentlichen Gesundheit für den Staat bildet. Explizit wird daran gemahnt, dass der Staat »nicht bloß die Si-

\footnotetext{
47 Von Stein (1882), 132.

48 Ebd., 133.

49 Ebd., 134.

50 Ebd.

51 Ebd., 133.

52 Ebd.
} 
cherheit und nicht bloß die Kraft seiner selbst in der seiner Bürger sucht, sondern sich selbst als die höchste Form der Ethik, der thätigen Sittlichkeit erkennt. « ${ }^{53}$ Der Gefahr einer Beschränkung der Gesundheitsrechte des Einzelnen im Interesse der Allgemeinheit ist mit dieser Begründung ein Riegel vorgeschoben. Deutlicher noch schützt das Prinzip der »freien Persönlichkeit« mit dem Verweis auf die »individuelle Freiheit« den Einzelnen gegen Übergriffe des Staates im Namen der öffentlichen Gesundheit und gegen staatliche Bevormundung im Sinne einer Pflicht zur Gesundheit: »Jeder soll vor allen Dingen und grundsätzlich der Hüter und Förderer seines eigenen Lebens, seiner eigenen Gesundheit sein. « ${ }^{54}$

Diese Doppelstruktur, die allgemeines und individuelles Wohl zueinander und zum Wohle des Staates in Beziehung setzt und zugleich die Freiheitsrechte des Einzelnen schützt, gerät zu Beginn des 20. Jahrhunderts aus dem Blickfeld. So knüpft die Sozialhygiene in der Weimarer Republik zwar explizit an die Überlegungen Virchows und Steins zu einem universellen Recht auf Gesundheit an, läuft aber Gefahr, in ihrem Kampf um die öffentliche Gesundheit die Freiheitsrechte des Einzelnen zu vernachlässigen und Gesundheitsrechte durch Gesundheitspflichten zu ergänzen. ${ }^{55}$

In seinem Aufsatz »Neue Fragestellungen auf dem Gebiet der Hygiene« (1918) verweist der Sozialhygieniker Alfons Fischer hinsichtlich der Frage nach dem Ziel der Sozialhygiene neben Rudolf Virchow und Lorenz von Stein auch auf einen frühen Gesetzentwurf von Franz Anton Mai (1800) zum Recht auf Gesundheit. ${ }^{56}$ Die Sozialhygiene befasse sich »mit den Einflüssen der sozialen Umwelt auf die Gesundheitsverhältnisse « ${ }^{57}$, der Staat habe dabei dafür zu sorgen, dass »die Sorge der Gemeinschaft für die Bedingungen der Erhaltung der Gesundheit sowie für die Heilung von Krankheiten [...] nicht mehr von dem Besitze des Kapitals abhängig « sei. ${ }^{58}$ Wenige Jahre später veröffentlicht er eine Schrift zu »Gesundheitsrecht und Gesundheitspflicht« (1925), in der Rechte und Pflichten über die Kombina-

\section{Ebd.}

54 Von Stein (1882), 133.

55 Zur Sozialhygiene zu Beginn des 20. Jahrhunderts vgl. Heinzelmann (2009).

56 Vgl. Fischer (1918), 234, Mai (1802). Vgl. auch Fischer (1913) und Labisch (1992), 90.

57 Fischer (1918), 220.

58 Von Stein (1882); zitiert bei Fischer (1918), 234. 
tion individueller und sozialer Hygiene zusammenführt werden: »Hygiene ist eine Wissenschaft, die zum Gesundheitsrecht führen soll, und sie ist eine Tugend, zu der die Jugend zu erziehen ist, damit jeder Staatsbürger in Zukunft seine Gesundheitspflicht erfüllt. ${ }^{59}$

Zusammenfassend zeigt der Überblick über frühe Ansätze zu einem Recht auf Gesundheit, dass die zu seiner Realisierung notwendige Bindung an das für die öffentliche Gesundheit verantwortliche Gesundheitswesen die Gefahr menschenrechtsverletzender Zwangsmaßnahmen als radikale Möglichkeit der Krankheitsbekämpfung mit einschließt. Angesichts dieser systemimmanenten Gefahren ist, wie im Folgenden zu zeigen sein wird, die Einbindung des Rechts auf Gesundheit in den Gesamtkontext der freiheitlich ausgerichteten Menschenrechte entscheidend.

\section{DAS »RECHT AUF GESUNDHEIT« IM MENSCHENRECHTLICHEN KONTEXT}

In der Allgemeinen Erklärung der Menschenrechte (AEMR) von 1948 wird Gesundheit im Vergleich zur Definition der WHO deutlich enger gefasst und als Bestandteil angemessener Lebensqualität von anderen Formen des Wohls unterschieden. ${ }^{60}$ Krankheit wird hier in den Kontext eines Rechts auf Sicherheit eingeordnet. Im Vordergrund steht die soziale Absicherung im Falle krankheitsbedingter Erwerbsunfähigkeit. Von einem Recht auf angemessene Gesundheitsversorgung ist hier noch nicht explizit die Rede. Rechtsverbindlich und als eigenständiges Recht wird das »Recht auf Gesundheit« erst in Art. 12 des UN-Sozialpakts von 1966 ausgeführt. ${ }^{61}$ Die Vertragsstaaten erkennen darin, wie es gleich zu Beginn in der Definition heißt, »das Recht eines jeden auf das für ihn erreichbare Höchstmaß an

59 Fischer (1925), 52.

60 »Everyone has the right to a standard of living adequate for the health and wellbeing of himself and of his family, including food, clothing, housing and medical care and necessary social services, and the right to security in the event of unemployment, sickness, disability, widowhood, old age or other lack of livelihood in circumstances beyond his control.« Universal Declaration of $\mathrm{Hu}-$ man Rights, Art. 25. 1.

61 Vgl. Krennerich (2013), 402. 
körperlicher und geistiger Gesundheit an.« Dabei werden Unterschiede, sowohl in der biologischen Konstitution als auch in der sozioökonomischen Ausgangssituation und Lebensweise verschiedener Individuen, in der Formulierung »höchstmöglich« explizit reflektiert. Im Jahre 2000 verabschiedete das UN Committee on Economic, Social and Cultural Rights den Allgemeinen Kommentar zum Recht auf Gesundheit, der das Recht auf Gesundheit auf der Basis der AEMR in verschiedene Richtungen präzisiert. Unter anderem beinhaltet Art. 12 laut Kommentar das Recht »to the enjoyment of a variety of facilities, goods, services and conditions necessary for the realization of the highest attainable standard of health « ${ }^{62}$. Im Zentrum steht in diesem Passus die Verpflichtung des Staates, Versorgungsleistungen und Institutionen bereitzustellen, die es jedem Einzelnen ermöglichen, den für ihn höchstmöglichen Zustand an Gesundheit zu erlangen. Ein Anspruch auf vollständige Gesundheit oder gar eine Bürgerpflicht, gesund zu sein und zu bleiben, sind nicht nur kein Bestandteil des menschenrechtlich verankerten Rechts auf Gesundheit, sie würden ihm vielmehr geradezu zuwiderlaufen. $^{63}$

Zwei entscheidende konzeptuelle Aspekte unterscheiden dieses »Recht auf Gesundheit« von seinen historischen Vorläufern: Es steht erstens nicht für sich allein, sondern ist in die gesamtmenschenrechtliche Systematik eingebettet. Maßnahmen des Staates zur Förderung der öffentlichen Gesundheit sind daher auf ihre Verträglichkeit mit den anderen Menschenrechten hin zu prüfen. Zweitens, aber keineswegs weniger wichtig, ist der in der Präambel der AEMR (1948) ausformulierte Rückbezug aller Menschenrechte auf die Menschenwürde. Die Anerkennung einer jedem Menschen inhärenten Würde bildet den Grund aller Menschenrechte und garantiert zugleich, dass diese Rechte jedem Einzelnen, ohne Ausnahme, allein aufgrund seines Menschseins zugesprochen werden müssen. Umgekehrt dienen die einzelnen Menschenrechte ihrerseits der expliziten Anerkennung

62 CESCR E/C.12/2000/4, 11. August 2000, Ziff. 9.

$63 »[\ldots]$ good health cannot be insured by a State, nor can States provide protection against every possible cause of human ill health. Thus, genetic factors, individual susceptibility to ill health and the adoption of unhealthy or risky lifestyles may play an important role with respect to an individual's health.« CESCR E/C.12/2000/4, 11. August 2000, Ziff. 9. 
der Menschenwürde. ${ }^{64}$ Die Achtung und Beförderung der Menschenwürde gehören demnach zum Kern des »Rechts auf Gesundheit«. Anders formuliert: Zu diesem Recht gehört, dass Maßnahmen des Staates zur Verbesserung der Gesundheit mit der Menschenwürde vereinbar sein müssen.

Das ist auch Juli Zeh als studierter Juristin bekannt. In Corpus Delicti findet sich daher nun mit Bezug auf die AEMR eine zweite folgenreiche Verschiebung. Die Bedeutung der Menschenwürde für die Menschenrechte und auch für das bundesrepublikanische Grundgesetz wird durch ihre Position innerhalb zentraler Dokumente hervorgehoben. So erscheint das Gebot ihrer Anerkennung gleich im ersten Satz der Präambel der AEMR: »Whereas the recognition of the inherent dignity and of the equal and inalienable rights of all members of the human family is the foundation of freedom, justice and peace in the world.« Im 1. Artikel des bundesrepublikanischen Grundgesetzes heißt es: »Die Würde des Menschen ist unantastbar.« Das entsprechende Dokument des Romans spielt sowohl auf die Präambel der AEMR als auch auf den 1. Artikel des Grundgesetzes an, ersetzt jedoch den Begriff der Würde durch den des Wohls: »Die METHODE dient dem Wohl des Menschen, Artikel eins der Präambel «. ${ }^{65}$ Die Konsequenz dieser Verabsolutierung der Gesundheit unter Verabschiedung der Menschenwürde ist die Aufhebung des Menschenrechtsschutzes. Menschenrechte adressieren den Staat und nehmen diesen in die Pflicht, bestimmte Standards zu gewährleisten, sie dienen jedoch zugleich dem Schutz des Einzelnen vor staatlichen und globalen gesundheitspolitischen Übergriffen.

Der Respekt vor der Menschenwürde des Einzelnen erweist sich damit zugleich auch als Voraussetzung von Autonomie und Selbstbestimmung. Setzt man das auch nach medizinischen Kriterien von Dritten definierbare Wohl des Patienten an die Stelle der ihm inhärenten Würde, so entfallen die Schranken, die den Einzelnen als autonomes Individuum vor übergeordneten gesundheitspolitischen und biomedizinischen Interessen schützen. Aus der Perspektive des »allgemeinen Wohl[s]« ist Selbstbestimmtheit ein »untragbarer Kostenfaktor ${ }^{66}{ }^{6}$ Ohne diese Orientierung am Würdebegriff ließe sich das Recht auf Gesundheit für sämtliche Maßnahmen zur Beförderung der öffentlichen Gesundheit missbrauchen.

64 Vgl. Bielefeldt (2011), 105-116.

65 Zeh (2010), 110.

66 Ebd., 187. 


\section{FAZIT}

Menschenrechte sind neben Anspruchsrechten vor allem auch Freiheitsrechte. Sie sollen die größtmögliche Autonomie (im Sinne von Selbständigkeit) und die freie Selbstbestimmung des Einzelnen stärken, sowohl durch den Schutz vor »freiheitseinschränkenden Eingriffen durch staatliche Organe und Dritte« als auch durch »die Schaffung der realen Bedingungen, die autonomes, selbstbestimmtes Handeln erst ermöglichen. $"{ }^{67}$ Diese freiheitliche Ausrichtung der Menschenrechte - Respekt der Privatsphäre, selbständige Lebensgestaltung, freie Ehegattenwahl, Meinungsäußerungs- und Informationsfreiheit etc. - prägt auch die Konturen des darin eingebunden Rechts auf Gesundheit.

Ein wichtiges Instrument zur Sicherung der Freiheitsrechte insgesamt besteht in der Abwägung der Verhältnismäßigkeit von Maßnahmen, die zur Gewährleistung eines spezifischen Menschenrechts durchgeführt werden, hinsichtlich ihrer möglichen Einschränkungen anderer Menschenrechte. Bezüge zwischen den Menschenrechten herzustellen, ist Aufgabe der Allgemeinen Kommentare zu den einzelnen Menschenrechten. So setzt der Allgemeine Kommentar Nr. 14 zum Recht auf Gesundheit dieses Recht in Beziehung zum Recht auf Achtung des Privatlebens (wie es beispielsweise in Artikel 8 der EMRK verankert ist). Das dort enthaltene "Selbstbestimmungsrecht über den eigenen Körper« umfasst u.a. den »Schutz der physischen und psychischen Integrität des Einzelnen«. Als »Recht auf körperliche Unversehrtheit« (Art 2.2 GG) und allgemeine Entscheidungsfreiheit (Art. 2.1. GG) geht es in das bundesrepublikanische Grundgesetz ein. ${ }^{68} \mathrm{Als}$ Recht auf Behandlungs- oder Therapieverzicht konkretisiert, geht das Recht auf Achtung des Privatlebens in den Allgemeinen Kommentar Nr. 14 zum Recht auf Gesundheit ein:

67 Krennerich (2013), 336-337.

68 Vgl. mit etwas anderer Fokussierung Krennerich (2013), 205: »Der Schutz der körperlichen Unversehrtheit kann somit, obwohl hierzulande getrennt ausgewiesen und konkretisiert, als Bestandteil des Rechts auf Gesundheit angesehen werden.« 
»The right to control one's health and body, including sexual and reproductive freedom, and the right to be free from interference, such as the right to be free from torture, non-consensual medical treatment and experimentation. « ${ }^{69}$

Mit seinem Schutz vor nicht-einvernehmlichen (non-consensual) medizinischen Behandlungen und Experimenten sowie der Anerkennung des Selbstbestimmungsrechts des Einzelnen über die persönliche Gesundheit und den eigenen Körper beinhaltet dieser Passus des Kommentars ein Abwehrrecht des Einzelnen gegenüber ärztlichen Eingriffen ebenso wie gegenüber staatlicher oder globaler gesundheitspolitischer Bevormundung.

Auch im menschenrechtlichen Kontext hat das Recht auf Behandlungsund Therapieverzicht jedoch seine Grenzen. Zwangsmedikationen und -behandlungen sind nicht gänzlich ausgeschlossen. Erlaubt sind Einschränkungen dort, wo die Gefahr einer gesundheitlichen Schädigung Dritter oder (in schwerwiegenden Fällen) auch des Kranken selbst vorliegt. So ist der Staat mit Blick auf die öffentliche Gesundheit dazu berechtigt, zum Schutz der Bevölkerung vor übertragbaren Krankheiten und Epidemien kollektive Maßnahmen wie obligatorische Impfungen, Quarantäneverordnungen, aber auch Röntgenuntersuchungen größerer Bevölkerungsgruppen anzuordnen und auch gegen den Willen des Einzelnen durchzusetzen. ${ }^{70}$ Mit Blick auf die Gefahren des Missbrauchs solcher Einschränkungen der Freiheitsrechte im Namen der öffentlichen Gesundheit - neben dem Recht auf Behandlungsverzicht sind hier insbesondere Einschränkungen der Religionsfreiheit, der Meinungsfreiheit und der Versammlungsfreiheit betroffen ${ }^{71}$ weist der Allgemeine Kommentar jedoch explizit auf die Notwendigkeit der Abwägung der Verhältnismäßigkeit des Eingriffs im Vergleich zur Schwere der Bedrohung öffentlicher Gesundheit hin. ${ }^{72}$ Das Prinzip der Verhältnis-

69 CESCR E/12/2000/4, 11. August 2000, Ziff. 8.

70 Gerichtsentscheidungen finden sich in der »Global Health and Human Rights Database« - zur obligatorischen Röntgenuntersuchung bei Tuberkuloseverdacht: Decision 2012/B/1991 in the Name of the Republic of Hungary; zur obligatorischen Impfung: Decision No. 362/10, Granada.

71 Vgl. die jeweiligen Passagen im CCPR, Ziff. 18, 19, 21 sowie die Art. 9, 10 und 11 der EMRK.

72 Vgl. CESCR E/C.12/2000/4, 11. August 2000, Ziff. 28. »Issues of public health are sometimes used by States as grounds for limiting the exercise of other 
mäßigkeit impliziert dabei eine Verteilung der Argumentationslast in Richtung auf diejenigen, die Freiheitseinschränkungen für erforderlich halten.

Die zweite Einschränkung des Abwehrrechts medizinischer Behandlung gilt dem Schutz des Kranken selbst. Sie ist in den letzten Jahren insbesondere im Kontext der Zwangsmedikation mit Neuroleptika in der Psychiatrie zur Behandlung akuter Psychosen kontrovers diskutiert und insgesamt zugunsten einer Stärkung der Patientenautonomie entschieden worden. An dieser Debatte werden die ethischen Probleme deutlich, die sich sowohl aus der Einschränkung der Patientenautonomie zugunsten der ärztlichen Fürsorgepflicht auf der Basis einer medizinischen Indikation ergeben als auch umgekehrt aus der Forderung einer Stärkung der Patientenautonomie bis hin zu einer »Freiheit zur Krankheit «. ${ }^{73}$

Insgesamt lässt sich die eingangs gestellte Frage nach konzeptionellen und rechtlichen Sicherungen zur Verhinderung eines Missbrauchs des Rechts auf Gesundheit positiv beantworten. Aufgrund seiner Einbindung in den Gesamtkontext der Menschenrechte und der Fundierung der Menschenrechte durch die jedem Menschen inhärente Würde in der Präambel der Allgemeinen Erklärung der Menschenrechte ist das »Recht auf Krankheit» im Sinne eines Rechts auf ungesunde Lebensführung und auf die Entscheidung zur Nicht-Behandlung dem Art. 12. des UN-Sozialpakts zum »Recht auf Gesundheit« eingeschrieben. Auch dort, wo die Freiheit zur Krankheit eingeschränkt wird, besteht die Möglichkeit, gegen eine unverhältnismä-

fundamental rights. The Committee wishes to emphasize that the Convenant's limitation clause, article 4 , is primarily intended to protect the rights of individuals rather than to permit the imposition of limitations by States. « Sowie ebd., Ziff. 29. »In line with article 5.1, such limitations must be proportional, i.e. the least restrictive alternative must be adopted where several types of limitations are available. Even where such limitations on grounds of public health are basically permitted, they should be of limited duration and subject to review."

73 Verwendung findet die Formulierung »Freiheit zur Krankheit« u.a. in den Urteilsbegründungen des Bundesverfassungsgerichts: BVerfGE 58. 208 [226]; BVerfG 2. Senat, 23. März 2011, Az: 2 BvR 882/09, 2., 16, Nr. 55. Zusammenfassend zur Rechtslage Müller et al. (2012), 1142-1149; zu den Folgen für die klinische Praxis ebd., 1150-1155; Konrad/Müller (2015), 897-905; zur ethischen Analyse der neuen Rechtslage Vollmann (2014), 614-620. 
ßige Einschränkung der Freiheiten des Einzelnen im Namen der öffentlichen Gesundheit sowie gegen paternalistische Fürsorge rechtlich Einspruch zu erheben.

Zehs Roman zeigt allerdings, dass diese Verbindung des Rechts auf Gesundheit mit der Freiheit zur Krankheit angesichts neuer Entwicklungen im Gesundheitswesen, aktuell insbesondere im Kontext des Datenschutzes und der zunehmenden Bedeutung der Krankheitsprävention und der Molekulargenetik, immer wieder neu verhandelt, verteidigt und gegebenenfalls erstritten werden muss. Die breite Tragweite leichter Verschiebungen in den Formulierungen - von einer umfassenden zu einer vollkommenen Gesundheit im Vergleich der Gesundheitsdefinition der WHO und derjenigen des Romans - führt zudem eindringlich vor, wie leicht Bestrebungen zu einer Optimierung der Gesundheit eine für die demokratische Freiheit ungünstige Wendung nehmen können. Welche Folgen die Unterschätzung institutionell eingeführter Normen für das komplexe Gefüge der Menschenrechte in ihrem Zusammenspiel haben kann, wird an der Ersetzung der Würde des Menschen als Grundlage der Menschenrechtskonvention durch das Wohl des Menschen, mit dem Zehs totalitärer Staat sich im Namen der öffentlichen Gesundheit legitimiert, als Gedankenexperiment anschaulich inszeniert.

\section{LITERATUR}

Bauman, Zygmunt (1994): Tod, Unsterblichkeit und andere Lebensstrategien, Frankfurt: Fischer.

Bielefeldt, Heiner (2011): Auslaufmodell Menschenwürde? Warum sie in Frage steht und warum wir sie verteidigen müssen, Freiburg i.Br.: Herder.

Bielefeldt, Heiner/Deile, Volkmar/Hamm, Brigitte/Hutter, Franz-Josef/ Kurtenbach, Sabine/Tretter, Hannes (Hg.) (2011): Nothing to hide nothing to fear? Datenschutz, Transparenz, Solidarität. Jahrbuch Menschenrechte 2011, Wien u.a.: Böhlau.

Bröckling, Ulrich (2004): »Art. >Prävention««, in: Bröckling et al. (2004), 210-215.

Bröckling, Ulrich (2008): »Vorbeugen ist besser ... Zur Soziologie der Prävention«, in: Behemoth. A Journal of Civilisation 1 (2008), 38-48. 
Bröckling, Ulrich/Krasmann, Susanne/Lemke, Thomas (Hg.) (2004): Glossar der Gegenwart, Frankfurt/M.: Suhrkamp.

Brodnig, Ingrid (2009): »Menschen werden gezielt eingeschüchtert«, Futurzone.orf.at - Futurzone ORF (gesendet am 24. September 2009), Online: www.fuzo-archiv.at/ [21.12.2015].

Canguilhem, Georges (2013a): »Krankheiten [Les maladies, 1989]«, in: Canguilhem (2013b), 29-42.

Canguilhem, Georges (2013b): Schriften zur Medizin. Mit einem Nachwort versehen von Michael Hagner, Zürich: diaphanes.

Chilese, Viviana/Preusser, Heinz-Peter (Hg.) (2013): Technik in Dystopien, Heidelberg: Winter.

Clausen, Jens/Levy, Neil (Eds.) (2015): Handbook of Neuroethics, Dordrecht u.a.: Springer.

Dabrock, Peter (2015): »Freiheit, Selbstbestimmung, Selbstkonstruktion zur Ethik von Big Data«, in: Deutscher Ethikrat (2015), Online: www.ethikrat.org/veranstaltungen/Jahrestagungen/die-vermessung-desmenschen [03.12.2015].

Deutscher Ethikrat (2015): Die Vermessung des Menschen - Big Data und Gesundheit, Online: www.ethikrat.org/veranstaltungen/Jahrestagungen/ die-vermessung-des-menschen [03.12.2015].

Fischer, Alfons (1913): Ein sozialhygienischer Gesetzentwurf aus dem Jahre 1800, ein Vorbild für die Gegenwart, Berlin: Springer.

Fischer, Alfons (1918): „Neue Fragestellungen auf dem Gebiet der Hygiene«, in: Lesky (1977), 217-249.

Fischer, Alfons (1925): »Gesundheitsrecht und Gesundheitspflicht«, in: Sozialhygienische Mitteilungen 9, 1/2 (1925), 44-52.

Frank, Johann Peter (1779-1788): System einer vollständigen medicinischen Polizey. 4 Bde., Mannheim: Schwan und Götz.

Frank, Johann Peter (1790): Akademische Rede vom Volkselend als Mutter der Krankheiten, Übersetzt und mit einer Einleitung versehen von Erna Lesky, Leipzig: Barth [1960].

Frevert, Ute (1984): Krankheit als politisches Problem 1770-1880. Soziale Unterschichten in Preußen zwischen medizinischer Polizei und staatlicher Sozialversicherung, Göttingen: Vandenhoeck \& Ruprecht.

Geisenhanslüke, Achim (2013): »Die verlorene Ehre der Mia Holl. Juli Zehs Corpus Delicti«, in: Chilese/Preusser (2013), 223-232. 
Hardy, Anne (2005): Ärzte, Ingenieure und städtische Gesundheit. Medizinische Theorien in der Hygienebewegung des 19. Jahrhunderts, Kultur der Medizin, Bd. 17, Frankfurt/M., New York: Campus Verlag.

Heinzelmann, Wilfried (2009): Sozialhygiene als Gesundheitswissenschaft. Die deutsch/deutsch-jüdische Avantgarde 1897-1933. Eine Geschichte in sieben Profilen, Bielefeld: transcript.

Hilmes, Carola (2013): »Frei von Leid und Schmerz. Juli Zehs literarische Version einer Gesundheitsdiktatur«, in: Forschung Frankfurt. Thema: Der korrigierte Mensch 1 (2013), 70-71 (Rezension), Online: www. forschung-frankfurt.uni-frankfurt.de [27.11.2015].

Konersmann, Ralf (Hg.) (2007): Wörterbuch der philosophischen Metaphern, Darmstadt: Wissenschaftliche Buchgesellschaft.

Konrad, Norbert/Müller, Sabine (2015): »Compulsory Interventions in Mentally Ill Persons at Risk of Becoming Violent«, in: Clausen/Levy (2015), 879-905.

Krennerich, Michael (2013): Soziale Menschenrechte. Zwischen Recht und Politik, Schwalbach/Ts.: Wochenschau-Verlag.

Krüger-Brand, Heike E. (2014): »Mobile Health: Fitness- und Gesundheitswächter«, in: Ärzteblatt 111 (2014), 45, Online: www.aerzteblatt. de/archiv/163387/Mobile-Health-Fitness-und-Gesundheitswächter [18. 10.2015].

Kunnert, Gerhard (2011): »Der vernetzte selektronische Patient««, in: Bielefeldt et al. (2011), 132-149.

Labisch, Alfons (1992): Homo Hygienicus. Gesundheit und Medizin in der Neuzeit, Frankfurt/M., New York: Campus.

Lengwiler, Martin/Madarász, Jeannette (Hg.) (2010): Das präventive Selbst. Eine Kulturgeschichte moderner Gesundheitspolitik, Bielefeld: transcript.

Lesky, Erna (Hg.) (1977): Sozialmedizin. Entwicklung und Selbstverständnis, Darmstadt: Wissenschaftliche Buchgesellschaft.

Lüdemann, Susanne (2007): »Art. `Körper, Organismus««, in: Konersmann (2007), 168-182.

Mai, Anton (1802): Stolpertus, der Polizeiarzt im Gerichtshof der medizinischen Polizeigesetzgebung, von einem patriotischen Pfälzer, Vierter Teil, Mannheim: Schwan und Götz [anonym erschienen]. 
Maierhofer, Waltraud (2012): »Juli Zeh’s Corpus Delicti (2009): Health Care, Terrorists, and the Return of the Political Message«, in: Monatshefte 104, 3 (2009), 375-392.

Müller, Klaus E. (Hg.) (2003): Phänomen Kultur. Perspektiven und Aufgaben der Kulturwissenschaften, Bielefeld: transcript.

Müller, Sabine/Walter, Henrik/Kunze, Heinrich/Konrad, Norbert/Heinz, Andreas (2012): »Zwangsbehandlung unter Rechtsunsicherheit. Teil 1: Die aktuelle Rechtslage zu Zwangsbehandlungen einwilligungsunfähiger Patienten mit psychischen Erkrankungen«, in: Der Nervenarzt 83 (2012), 1142-1155.

Röber, Friedrich August (1806): Von der Sorge des Staats für die Gesundheit seiner Bürger, Dresden: Gärtner.

Roelcke, Volker (2003): »Medizin - eine Kulturwissenschaft? Wissenschaftsverständnis, Anthropologie und Wertsetzungen in der modernen Heilkunde«, in: Müller (2003), 107-130.

Sarasin, Philippe (2001): Reizbare Maschinen. Eine Geschichte des Körpers 1765-1914, Frankfurt/M.: Suhrkamp.

Schulz, Frank (1987): Das »Recht auf Gesundheit«. Seine wirtschafts- und sozialpolitischen Voraussetzungen in der Geschichte der sozialmedizinischen Lehrmeinungen, Regensburg: transfer.

Virchow, Rudolf (1848/1983): »Die öffentliche Gesundheitspflege«, in: Virchow, Rudolf/Leubuscher, Rudolf (Hg.): Die medizinische Reform. Eine Wochenschrift, 1. und 2. Jg., 1948/49, No. 5, No. 7., No. 8, No. 9, 1848. Neudruck, Berlin (DDR) 1983, 21-22, 37-40, 45-47, 53-56.

Vollmann, Jochen (2014): »Zwangsbehandlungen in der Psychiatrie. Eine ethische Analyse der neuen Rechtslage für die klinische Praxis«, in: Nervenarzt 85 (2014), 614-620.

Von Stein, Lorenz (1882): Das Gesundheitswesen. Erstes Hauptgebiet, zweiter Teil der Inneren Verwaltungslehre. Zweite Auflage. Gänzlich neubearbeitet und bis auf die neueste Zeit verfolgt, Stuttgart: Cotta.

Wolff, Eberhard (2010): „Diätetik als Selbsttechnologie: Zum Verhältnis heteronomer und autonomer Selbstdisziplinierung zwischen Lebensreformbewegung und heutigem Gesundheitsboom«, in: Lengwiler et al. (2010), 169-204.

World Health Organization (1978): Erklärung von Alma-Ata, Online: www.euro.who.int/de/publications/policy-documents/declaration-ofalma-ata,-1978 [19.10.2015]. 
World Health Organization (1986): Ottawa Charta zur Gesundheitsförderung, Online: www.euro.who.int/de/publications/policy-documents/ottawacharter-for-health-promotion,-1986 [19.10.2015].

World Health Organization (2009): Constitution of the World Health Organization as adopted by the International Health Conference, New York, 19-22 June, 1946; signed on 22 July 1946 by the representatives of 61 States (Official Records of the World Health Organization, no. 2, p. 100) and entered into force on 7 April 1948, in: WHO: Basic Documents. Fourty-seventh Edition 2009, 1-18.

World Health Organization (2013a): Gesundheit 2020: Europäisches Rahmenkonzept für gesamtstaatliches und gesamtgesellschaftliches Handeln zur Förderung von Gesundheit und Wohlbefinden, Online: www.euro.who.int/en/health-topics/health-policy/health-2020-the-european-policy-for-health-and-well-being/publications/2013/health-2020a-european-policy-framework-supporting-action-across-governmentand-society-for-health-and-well-being [18.10.2015].

World Health Organization (2013b): Erklärung von Aschgabat. Über die Prävention und Bekämpfung nichtübertragbarer Krankheiten im Kontext von Gesundheit 2020, Online: www.euro.who.int/data/assets/pdf_ file/0003/236190/Ashgabat-Declaration-4-December-2013-Ger.pdf [18. 10.2015].

Zeh, Juli (2010): Corpus Delicti. Ein Prozess, München: btb. Erstauflage: Frankfurt/M.: Schöffling \& Co, 2009. 
Anhang 



\section{Allgemeine Bemerkung Nr. 14: \\ Das Recht auf ein Höchstmaß an Gesundheit (Artikel 12)}

Zweiundzwanzigste Sitzung (2000), E/C.12/2000/4

UN-AUSSCHUSS FÜR WIRTSCHAFTLICHE, SOZIALE UND KULTURELLE RECHTE

1. Gesundheit ist ein fundamentales Menschenrecht, das für die Ausübung der anderen Menschenrechte unerlässlich ist. Jeder Mensch ist berechtigt, ein Höchstmaß an Gesundheit zu erreichen, um ein Leben in Würde zu führen. Die Verwirklichung des Rechts auf Gesundheit kann mit verschiedenen, einander ergänzenden Ansätzen verfolgt werden. Hierzu gehören die Formulierung gesundheitspolitischer Richtlinien oder die Einführung von Gesundheitsprogrammen, die durch die Weltgesundheitsorganisation (WHO) entwickelt wurden, oder die Verabschiedung spezifischer rechtlicher Instrumente. Das Recht auf Gesundheit enthält darüber hinaus einige Elemente, die rechtlich durchsetzbar sind. ${ }^{1}$

2. Das Recht auf Gesundheit wird von zahlreichen internationalen Abkommen anerkannt. Artikel 25 Abs. 1 der Allgemeinen Erklärung der Men-

1 Das Diskriminierungsverbot ist zum Beispiel in Bezug auf medizinische Einrichtungen und ärztliche Betreuung in zahlreichen Ländern rechtlich durchsetzbar. 
schenrechte bekräftigt: »Jeder hat das Recht auf einen Lebensstandard, der seine und seiner Familie Gesundheit und Wohl gewährleistet, einschließlich Nahrung, Kleidung, Wohnung, ärztliche Versorgung und notwendige soziale Leistungen.« Der Internationale Pakt über Wirtschaftliche, Soziale und Kulturelle Rechte enthält den umfassendsten Artikel über das Recht auf Gesundheit im internationalen Menschenrechtsschutz. Gemäß Artikel 12 Abs. 1 des Pakts erkennen die Vertragsstaaten »das Recht eines jeden auf das für ihn erreichbare Höchstmaß an körperlicher und geistiger Gesundheit« an, während Artikel 12 Abs. 2 zur Erläuterung eine Anzahl von Schritten aufzählt, »die von den Vertragsstaaten zur vollen Verwirklichung dieses Rechts zu unternehmen sind «. Daneben wird das Recht auf Gesundheit etwa in Artikel 5 (e) (iv) des Internationalen Übereinkommens zur Beseitigung jeder Form der Rassendiskriminierung aus dem Jahr 1965 anerkannt, in den Artikeln 11 Abs. 1 (f) und 12 des Übereinkommens zur Beseitigung jeder Form von Diskriminierung der Frau aus dem Jahr 1979 und in Artikel 24 des Übereinkommens über die Rechte des Kindes aus dem Jahr 1989. Verschiedene regionale Menschenrechtsabkommen erkennen darüber hinaus das Recht auf Gesundheit an, zum Beispiel die Europäische Sozialcharta in der revidierten Fassung aus dem Jahr 1961 (Artikel 11), die Afrikanische Charta der Menschenrechte und Rechte der Völker aus dem Jahr 1981 (Artikel 16) und das Zusatzprotokoll zur Amerikanischen Menschenrechtskonvention über wirtschaftliche, soziale und kulturelle Rechte aus dem Jahr 1988 (Artikel 10). In ähnlicher Weise ist das Recht auf Gesundheit von der Menschenrechtskommission, ${ }^{2}$ in der Wiener Erklärung und im Aktionsprogramm aus dem Jahr 1993 und anderen internationalen Abkommen proklamiert worden. ${ }^{3}$

2 In ihrer Resolution 1989/11.

3 Die Grundsätze für den Schutz von psychisch Kranken und die Verbesserung der psychiatrischen Versorgung, verabschiedet durch die Generalversammlung der Vereinten Nationen im Jahr 1991 (Resolution 46/119) und die Allgemeine Bemerkung Nr. 5 über Menschen mit Behinderungen sind auf Menschen mit psychischen Krankheiten anzuwenden; das Aktionsprogramm der Internationalen Konferenz über Bevölkerung und Entwicklung, die im Jahr 1994 in Kairo stattgefunden hat, ebenso wie die Erklärung und das Aktionsprogramm der vierten Weltfrauenkonferenz, die in Peking im Jahr 1995 stattgefunden hat, enthal- 
3. Das Recht auf Gesundheit steht in enger Beziehung zu und ist abhängig von der Verwirklichung anderer Menschenrechte, wie sie in der International Bill of Rights enthalten sind, einschließlich der Rechte auf Nahrung, Wohnung, Arbeit, Bildung, menschliche Würde, Leben, Diskriminierungsverbot, Gleichheit, Folterverbot, Privatsphäre, Zugang zu Information, Vereinigungsfreiheit, Versammlungsfreiheit und Freizügigkeit. Diese und andere Rechte und Freiheiten stellen integrale Bestandteile des Rechts auf Gesundheit dar.

4. Bei der Erarbeitung des Artikel 12 des Pakts hat der Dritte Ausschuss der UN-Generalversammlung nicht die Definition von Gesundheit übernommen, die in der Präambel der Satzung der WHO enthalten ist und die Gesundheit als einen »Zustand vollständigen körperlichen, geistigen und sozialen Wohlbefindens und nicht nur als Abwesenheit von Krankheit oder Gebrechlichkeit« beschreibt. Die Verweisung des Artikels 12 Abs. 1 des Pakts auf »das erreichbare Höchstmaß an körperlicher und geistiger Gesundheit« ist zugleich nicht auf das Recht auf Gesundheitsfürsorge beschränkt. Im Gegenteil, die Entstehungsgeschichte und der ausdrückliche Wortlaut von Artikel 12 Abs. 2 lassen erkennen, dass das Recht auf Gesundheit weitreichende soziale und wirtschaftliche Faktoren umfasst, aufgrund derer Menschen ein gesundes Leben führen können, und dass es sich auch auf die zugrunde liegenden Parameter der Gesundheit, wie Nahrung und Ernährung, Wohnung, Zugang zu sicherem Trinkwasser und angemessenen Sanitäreinrichtungen, sichere und gesunde Arbeitsbedingungen und eine gesunde Umwelt erstreckt.

5. Der Ausschuss ist sich bewusst, dass die volle Beanspruchung des Rechts auf Gesundheit für Millionen von Menschen auf der ganzen Welt noch immer ein fernes Ziel bleibt. Überdies rückt dieses Ziel für diejenigen, die in Armut leben, in zunehmend größere Ferne. Der Ausschuss erkennt die gewaltigen strukturellen und sonstigen Hindernisse an, die sich aus Faktoren jenseits der Kontrolle der Staaten ergeben und die die volle Verwirklichung des Artikel 12 in vielen Vertragsstaaten behindern.

ten jeweils Definitionen im Hinblick auf Familienplanung und die Gesundheit der Frau. 
6. Mit der Absicht, den Vertragsstaaten bei der Umsetzung des Pakts und bei der Erfüllung ihrer Verpflichtungen bei der Berichterstattung zu helfen, konzentriert sich diese Allgemeine Bemerkung auf den normativen Inhalt des Artikel 12 (Teil I), die Verpflichtungen der Vertragsstaaten (Teil II), Verletzungen (Teil III) und die Umsetzung auf innerstaatlicher Ebene (Teil IV), während Verpflichtungen von nichtstaatlichen Akteuren in Teil V angesprochen werden. Die Allgemeine Bemerkung beruht auf der Erfahrung des Ausschusses, die er bei der Prüfung der Staatenberichte im Laufe mehrere Jahre gewonnen hat.

\section{DeR NORMATIVE INHALt VON ARTIKEL 12}

7. Artikel 12 Abs. 1 definiert das Recht auf Gesundheit, während Artikel 12 Abs. 2 einige erläuternde, jedoch nicht erschöpfende Beispiele zu den Verpflichtungen der Vertragsstaaten aufzählt.

8. Das Recht auf Gesundheit wird nicht als ein Recht darauf, gesund zu sein, verstanden. Das Recht auf Gesundheit enthält sowohl Freiheiten als auch Rechte. Die Freiheiten schließen das Recht ein, über die eigene Gesundheit und den eigenen Körper zu bestimmen, einschließlich der sexuellen und reproduktiven Freiheit, das Recht, frei von Eingriffen zu sein und wie das Recht, nicht misshandelt, nicht medizinischer Behandlung oder medizinischen Versuchen ohne Einwilligung unterzogen zu werden. Dagegen umfassen die Rechte unter anderem das Recht auf ein Gesundheitsfürsorgesystem, das die gleichberechtigte Erreichung eines Höchstmaßes an Gesundheit gewährleistet.

9. Der Begriff des »erreichbaren Höchstmaßes an Gesundheit« in Artikel 12 Abs.1 zieht sowohl die biologischen, sozialen und wirtschaftlichen Voraussetzungen eines Individuums als auch die verfügbaren Ressourcen eines Staates in Betracht. Es gibt eine Anzahl von Aspekten, die nicht nur zwischen Staat und Individuum gelöst werden können; insbesondere kann gute Gesundheit weder allein durch den Staat gewährleistet werden, noch sind Staaten imstande, den Menschen gegen jede mögliche Krankheitsursache zu schützen. Genetische Faktoren, individuelle Anfälligkeit und ungesunde oder riskante Lebensführung können für die Gesundheit eines Indivi- 
duums eine wichtige Rolle spielen. Folglich muss das Recht auf Gesundheit als ein Recht auf den Genuss unterschiedlicher medizinischer Einrichtungen und ärztlicher Betreuungsdienste sowie den Genuss sonstiger Umstände verstanden werden, die für die Verwirklichung eines Höchstmaßes an Gesundheit notwendig sind.

10. Seit der Verabschiedung der beiden Internationalen Pakte im Jahr 1966 hat sich die Gesundheitslage der Welt dramatisch verändert. Auch hat der Begriff der Gesundheit substantielle Veränderungen durchlaufen und wird umfassender gedeutet. Im Hinblick auf Gesundheit werden mehrere Faktoren in Betracht gezogen, darunter die Verteilung der Ressourcen und die Unterschiede der Geschlechter. Eine weitere Definition der Gesundheit berücksichtigt auch gesellschaftsbezogene Belange, wie Gewalt und bewaffneten Konflikt. ${ }^{4}$ Ferner haben bis dahin unbekannte Krankheiten wie das menschliche Immunschwäche-Virus und das Immunschwäche-Syndrom (HIV/AIDS) sowie andere Krankheiten, die sich weit verbreitet haben (zum Beispiel Krebs), neue Hindernisse für die Verwirklichung des Rechts auf Gesundheit geschaffen, die bei der Auslegung des Artikel 12 berücksichtigt werden müssen. Hierzu ist ebenso das rasche Wachstum der Weltbevölkerung hinzuzurechnen.

11. Der Ausschuss interpretiert das Recht auf Gesundheit, wie es in Artikel 12 Abs. 1 definiert ist, als ein inklusives Recht, das sich nicht nur auf eine zeitgerechte und angemessene Gesundheitsfürsorge, sondern auch auf die zugrunde liegenden Parameter der Gesundheit erstreckt. Hierzu zählen der Zugang zu sauberem und gesundem Trinkwasser und adäquaten Sanitäranlagen, die angemessene Versorgung mit unbedenklicher Nahrung und Unterkunft, gesunde Arbeits- und Umweltbedingungen sowie der Zugang zu gesundheitsbezogener Ausbildung und Information, einschließlich der Aufklärung über Sexualgesundheit und reproduktive Gesundheit. Ein weiterer wichtiger Aspekt ist die Beteiligung der Bevölkerung an allen gesund-

4 Der gemeinsame Artikel 3 der Genfer Übereinkommen zum Schutz von Kriegsopfern (1949); Zusatzprotokoll I (1977), das sich auf den Schutz von Opfern internationaler bewaffneter Konflikte bezieht, Artikel 75 Abs. 2 (a); Zusatzprotokoll II (1977), das sich auf den Schutz von Opfern nicht-internationaler bewaffneter Konflikte bezieht, Artikel 4 (a). 
heitsbezogenen Entscheidungen auf kommunaler, nationaler und internationaler Ebene.

12. Das Recht auf Gesundheit in allen seinen Formen und auf allen Ebenen enthält folgende wesentliche und miteinander verbundene Elemente, deren genaue Anwendung von den in dem jeweiligen Staat vorherrschenden Bedingungen abhängt:

(a) Verfügbarkeit. In den Vertragsstaaten müssen funktionierende Einrichtungen der öffentlichen Gesundheitsfürsorge, medizinische Einrichtungen und ärztliche Betreuungsdienste ebenso wie Programme ausreichend verfügbar sein. Die konkrete Beschaffenheit der medizinischen Einrichtungen und ärztlichen Betreuungsdienste wird von zahlreichen Faktoren, einschließlich des Entwicklungsstands des Staats, abhängen. Sie werden jedoch die zugrunde liegenden Parameter der Gesundheit wie sicheres und sauberes Trinkwasser und adäquate Sanitäreinrichtungen, Krankenhäuser, Kliniken und andere gesundheitsbezogene Gebäude, geschultes medizinisches und anderes Personal, das ein im Inland wettbewerbsfähiges Gehalt bezieht, und unentbehrliche Arzneimittel, wie sie vom Aktionsprogramm über unentbehrliche Arzneimittel der WHO definiert sind, beinhalten; ${ }^{5}$

(b) Zugänglichkeit. Medizinische Einrichtungen und ärztliche Betreuung ${ }^{6}$ müssen für jeden Menschen innerhalb des Hoheitsgebiets eines Vertragsstaates ohne Diskriminierung zugänglich sein. Die Zugänglichkeit besitzt vier Dimensionen, die einander jeweils überlagern:

(i) Diskriminierungsverbot: Medizinische Einrichtungen und ärztliche Betreuung müssen für alle, insbesondere für die besonders schutzbedürftigen und an den Rand der Gesellschaft gedrängten Gruppen der

5 Vgl. die Liste unentbehrlicher Arzneimittel der WHO, revidiert im Dezember 1999, WHO Drug Information, vol.13, Nr. 4, 1999.

6 Jede Verweisung auf medizinische Einrichtungen und ärztliche Betreuung in dieser Bemerkung beinhaltet die zugrunde liegenden Gesundheitsparameter, die in Abs. 11 und 12 (a) dieser Allgemeinen Bemerkung näher erläutert werden. 
Bevölkerung, de iure und de facto ohne Verletzung des Diskriminierungsverbots zugänglich sein; ${ }^{7}$

(ii) Physische Zugänglichkeit: Medizinische Einrichtungen und ärztliche Betreuung müssen in sicherer Reichweite für die gesamte Bevölkerung vorhanden sein. Hierzu zählen insbesondere schutzbedürftige oder an den Rand gedrängte Gruppen wie ethnische Minderheiten und indigene Völker, Frauen, Kinder, Jugendliche, ältere Menschen, Menschen mit Behinderungen und Menschen mit HIV/AIDS. Zugänglichkeit beinhaltet auch, dass ärztliche Betreuung und die zugrunde liegenden Parameter für Gesundheit wie sicheres und sauberes Trinkwasser und adäquate Sanitäreinrichtungen innerhalb sicherer Reichweite liegen, was auch für ländliche Gebiete gilt. Zugänglichkeit schließt weiterhin behindertengerechten Zugang zu Gebäuden für Menschen mit Behinderungen ein;

(iii) Wirtschaftliche Zugänglichkeit (Bezahlbarkeit): Medizinische Einrichtungen und ärztliche Betreuung müssen für alle erschwinglich sein. Kosten für Dienstleistungen der Gesundheitsfürsorge ebenso wie für Leistungen, die sich auf die zugrunde liegenden Gesundheitsparameter beziehen, müssen auf dem Gerechtigkeitsgrundsatz beruhen und sicherstellen, dass diese Leistungen, ob öffentliche oder private, für alle, einschließlich sozial benachteiligter Gruppen, erschwinglich sind. Gerechtigkeit verlangt, dass ärmere Haushalte im Vergleich zu wohlhabenderen Haushalten nicht unverhältnismäßig mit Gesundheitsausgaben zu belasten sind;

(iv) Zugänglichkeit von Information: Zugänglichkeit beinhaltet das Recht, Informationen und Ideen ${ }^{8}$ im Hinblick auf Gesundheitsprobleme zu suchen, zu erhalten und weiterzugeben. Die Zugänglichkeit von In-

7 Vgl. Abs. 18 und 19 dieser Allgemeinen Bemerkung.

8 Vgl. Artikel 19 Abs. 2 des Internationalen Pakts über Bürgerliche und Politische Rechte. Diese Allgemeine Bemerkung hebt den Zugang zu Information wegen der speziellen Bedeutung dieses Aspekts in Zusammenhang mit Gesundheit hervor. 
formation sollte jedoch das Recht, dass persönliche Gesundheitsdaten mit Vertraulichkeit behandelt werden müssen, nicht beeinträchtigen;

(c) Annehmbarkeit. Medizinische Einrichtungen und ärztliche Betreuung müssen die medizinische Ethik achten und kulturell angemessen sein, das heißt die Kultur Einzelner, von Minderheiten, Völkern und Gemeinschaften achten, geschlechts- und altersbedingte Besonderheiten berücksichtigen, Vertraulichkeit achten und den Gesundheitszustand der Betroffenen verbessern;

(d) Qualität. Medizinische Einrichtungen und ärztliche Betreuung müssen nicht nur kulturell akzeptiert, sondern auch wissenschaftlich und medizinisch geeignet und von guter Qualität sein. Dies erfordert unter anderem geschultes medizinisches Personal, wissenschaftlich zugelassene und nicht veraltete Medikamente und Krankenhauseinrichtungen, sicheres Trinkwasser und adäquate Sanitäreinrichtungen.

13. Der nicht erschöpfende Katalog der Beispiele in Artikel 12 Abs. 2 gibt für die von den Staaten zu ergreifenden Maßnahmen eine Anleitung. Er gibt bestimmte allgemeine Beispiele für Maßnahmen, die aus der breiten Definition des Rechts auf Gesundheit in Artikel 12 Abs. 2 erwachsen. Dabei veranschaulichen sie den Inhalt dieses Rechts, wie in den folgenden Absätzen dargestellt wird. ${ }^{9}$

9 In der Literatur und Praxis, die sich auf das Recht auf Gesundheit bezieht, werden oft drei Ebenen der Gesundheitsfürsorge unterschieden: Primäre Gesundheitsfürsorge behandelt hauptsächlich allgemeine und weniger ernste Krankheiten, und sie wird von Fachpersonal des Gesundheitswesens und/oder praktischen Ärzten zur Verfügung gestellt, die innerhalb von Gemeinschaften zu relativ geringen Kosten arbeiten; sekundäre Gesundheitsfürsorge wird in Zentren, normalerweise in Krankenhäusern, angeboten, und sie behandelt typischerweise relativ verbreitete minder schwere oder schwere Krankheiten, die innerhalb der Gemeinschaft nicht behandelt werden können. Sie greift auf ein speziell ausgebildetes Fachpersonal des Gesundheitswesens und Ärzte zurück, auf spezielle Ausstattung und manchmal auf eine stationäre Pflege zu vergleichsweise höheren Kosten; tertiäre Gesundheitsfürsorge wird in relativ wenigen Zentren zur Verfügung gestellt, sie wird typischerweise für eine geringe Anzahl von Patienten 


\section{Artikel 12 Abs. 2 (a): Das Recht auf Gesundheit von Mutter und Kind sowie auf reproduktive Gesundheit}

14. »Die Vorschrift zur Senkung der Zahl der Totgeburten und der Kindersterblichkeit sowie zur gesunden Entwicklung des Kindes« (Artikel 12 Abs. 2 (a) ${ }^{10}$ kann als Erfordernis verstanden werden, für Maßnahmen zur Verbesserung der Gesundheit von Mutter und Kind zu sorgen, von Beratungsdiensten zur sexuellen und reproduktiven Gesundheit, von prä- und postnataler Fürsorge, ${ }^{11}$ Betreuung bei Notentbindungen sowie zur Verbesserung des Zugangs zu Informationen und zu den notwendigen Hilfsmitteln, um mit dieser Information umgehen zu können. ${ }^{12}$

mit minder schweren oder schweren Krankheiten angeboten, die ein speziell ausgebildetes Fachpersonal des Gesundheitswesens, Ärzte und spezielle Einrichtungen verlangen, und sie ist oft relativ teuer. Da die primäre, sekundäre und tertiäre Gesundheitsfürsorge sich oft überlagern und gegenseitig beeinflussen, stellt der Gebrauch dieser Typologie nicht immer hinreichend unterscheidende Kriterien zur Verfügung, um für die Einschätzung hilfreich zu sein, welche Form von Gesundheitsfürsorge Vertragsstaaten anbieten müssen. Deshalb ist sie in Bezug auf das normative Verständnis des Artikels 12 nur von beschränktem Nutzen.

10 Gemäß WHO wird nicht mehr die Rate der Totgeburten verwendet, stattdessen werden die Sterblichkeitsraten von Kindern unter fünf Jahren und der Säuglinge gemessen.

11 Pränatal bezeichnet »existierend oder auftretend vor der Geburt«; perinatal bezieht sich auf den Zeitraum kurz vor und nach der Geburt (in der medizinischen Statistik beginnt der Zeitraum mit der Vollendung von 28 Schwangerschaftswochen und wird definiert als zwischen einer Woche bis vier Wochen nach der Geburt endend); im Gegensatz dazu bezeichnet neonatal den Zeitraum, der sich auf die ersten vier Wochen nach der Geburt erstreckt; während postnatal das Auftreten nach der Geburt bezeichnet. In dieser Allgemeinen Bemerkung werden ausschließlich die allgemeineren Ausdrücke prä- und postnatal verwendet.

12 Familienplanung bedeutet, dass Frauen und Männer die Freiheit haben zu entscheiden, ob und wann sie zeugen wollen, und dass sie das Recht haben, informiert zu werden und Zugang zu sicheren, wirksamen, erschwinglichen und akzeptablen Methoden der Familienplanung zu haben. Ebenso haben sie das Recht 


\section{Artikel 12 Abs. 2 (b): Das Recht auf eine gesunde Umwelt und gesunde Arbeitsbedingungen}

15. „Die Verbesserung aller Aspekte der Umwelt- und der Arbeitshygiene» (Artikel 12 Abs. 2 (b)) umfasst unter anderem vorbeugende Maßnahmen im Hinblick auf Berufsunfälle und Berufskrankheiten; setzt voraus, angemessene Versorgung mit sauberem Trinkwasser und elementaren Sanitäranlagen zu gewährleisten; die Vorbeugung und Minderung des Risikos, verletzenden Substanzen wie Strahlung und gesundheitsschädlichen Chemikalien oder anderen schädlichen Umweltbedingungen ausgesetzt zu sein, die direkt oder indirekt die menschliche Gesundheit beeinflussen. ${ }^{13}$ Darüber hinaus bezieht sich die Arbeitshygiene, soweit dies praktikabel ist, auf eine Reduzierung der Ursachen von Gesundheitsrisiken, die die Arbeitsumgebung mit sich bringt. ${ }^{14}$ Artikel 12 Abs. 2 (b) umfasst auch eine angemessene Unterkunft und sichere und hygienische Arbeitsbedingungen, eine angemessene Versorgung mit Nahrungsmitteln sowie richtige Ernährung, und rät ab von Alkoholmissbrauch, dem Genuss von Tabak, Drogen und anderen schädlichen Substanzen.

auf Zugang zu angemessenen Gesundheitsdiensten, die zum Beispiel Frauen ermöglichen, die Schwangerschaft und Geburt sicher zu überstehen.

13 Der Ausschuss nimmt in diesem Zusammenhang das Prinzip 1 der Erklärung von Stockholm aus dem Jahr 1972 zur Kenntnis, das feststellt: »Der Mensch hat das fundamentale Recht auf Freiheit, Gleichheit und angemessene Lebensbedingungen in einer Umgebung, die ihm ein Leben in Würde und Wohlbefinden gestattet«. Er weist ebenso auf die zurückliegenden Entwicklungen im Völkerrecht hin, einschließlich der Resolution 45/94 der Generalversammlung über die Notwendigkeit, das Wohlbefinden der Menschen zu gewährleisten; Prinzip 1 der Erklärung von Rio; und regionale Menschenrechtsinstrumente wie Artikel 10 des Protokolls von San Salvador zur Amerikanischen Menschenrechtskonvention.

14 ILO-Übereinkommen, Nr. 155, Artikel 4 Abs. 2. 


\section{Artikel 12 Abs. 2 (c): Das Recht auf Vorbeugung, Behandlung und Bekämpfung von Krankheiten}

16. »Die Vorbeugung, Behandlung und Bekämpfung epidemischer, endemischer, Berufs- und sonstiger Krankheiten « (Artikel 12 Abs. 2 (c)) verlangen die Einführung von Präventions- und Aufklärungsprogrammen für verhaltensabhängige Gesundheitsbelange, wozu Krankheiten gehören, die durch Sexualverkehr übertragen werden, wie insbesondere HIV/AIDS, und diejenigen, die die sexuelle und reproduktive Gesundheit beeinträchtigen. Sie verlangen zudem die Förderung sozialer Bedingungen, die die Gesundheit beeinflussen, wie die Sicherheit der Umwelt, Bildung, wirtschaftliche Entwicklung und die Gleichberechtigung der Geschlechter. Das Recht auf Behandlung beinhaltet die Schaffung eines Systems für die Versorgung mit notärztlicher Betreuung bei Unfällen, Epidemien und ähnlichen Gesundheitsrisiken sowie die Versorgung mit einem Katastrophendienst und humanitärer Hilfe in Notsituationen. Die Bekämpfung von Krankheiten verlangt zum Beispiel Bemühungen der Staaten, einzeln oder gemeinsam, dazu benötigte Technologien verfügbar zu machen, eine epidemiologische Kontrolle und detaillierte Datenerhebung durchzuführen und zu verbessern sowie Immunisierungsprogramme und andere Strategien zur Bekämpfung von Infektionskrankheiten einzuführen oder zu verbessern.

\section{Artikel 12 Abs. 2 (d): Das Recht auf medizinische Einrichtungen und ärztliche Betreuung}

17. Die »Schaffung der Voraussetzungen, die für jeden Menschen im Krankheitsfall den Genuss medizinischer Einrichtungen und ärztlicher Betreuung sicherstellen «, und dies sowohl im Falle körperlicher als auch im Falle geistiger Krankheit (Artikel 12 Abs. 2 (d)), beinhaltet den gleichberechtigten und zeitgerechten Zugang zu elementaren Präventions-, Behandlungs- und Rehabilitationsleistungen sowie zu Gesundheitserziehung; eine regelmäßige Untersuchung; die angemessene Behandlung häufiger Krankheiten, Beschwerden, Verletzungen und Behinderungen, vorzugsweise auf Ebene der Kommunen; die Versorgung mit notwendigen Medikamenten und eine angemessene Behandlung und Pflege bei geistigen Krankheiten. Ein weiterer wichtiger Aspekt ist die Verbesserung und Förderung der Beteiligung der Bevölkerung an der Gesundheitsvorsorge und der Pflege, zum 
Beispiel durch eine Verbesserung der Organisation des Gesundheitssektors, des Versicherungssystems sowie insbesondere der Beteiligung der Bevölkerung an politischen Entscheidungen auf kommunaler und nationaler Ebene, die das Recht auf Gesundheit berühren.

\section{Artikel 12: Besondere Aspekte im Hinblick auf eine weite Anwendung}

\section{Diskriminierungsverbot und Gleichbehandlung}

18. Gemäß Artikel 2 Abs. 2 und Artikel 3 verbietet der Pakt jede Diskriminierung in der Gesundheitsfürsorge und den zugrunde liegenden Gesundheitsparametern (einschließlich des Zugangs zu Mitteln und zu Leistungsberechtigung bezüglich Gesundheitsfürsorge und Gesundheitsparameter) aufgrund von Rasse, Hautfarbe, Geschlecht, Sprache, Religion, politischer oder anderer Meinung, nationaler oder sozialer Herkunft, Eigentum, Geburt, körperlicher oder geistiger Behinderung, des Gesundheitsstatus (einschließlich HIV/AIDS), von sexueller Orientierung und bürgerlichem, politischem, sozialem oder sonstigem Status, der die Absicht oder die Wirkung hat, den gleichberechtigten Genuss oder die gleiche Ausübung des Rechts auf Gesundheit nichtig zu machen oder zu behindern. Der Ausschuss betont, dass viele Maßnahmen, zum Beispiel die meisten Strategien und Programme zur Beseitigung gesundheitsbezogener Diskriminierung, mit minimalem Aufwand verfolgt werden können, indem Gesetze verabschiedet, modifiziert oder aufgehoben werden, oder durch die Verbreitung von Information. Der Ausschuss ruft die Allgemeine Bemerkung Nr. 3 Abs. 12 in Erinnerung, die feststellt, dass selbst in Zeiten starker Mittelknappheit schutzbedürftige Mitglieder einer Gesellschaft durch Programme mit relativ geringem Kostenaufwand geschützt werden müssen.

19. Im Hinblick auf das Recht auf Gesundheit muss der gleichberechtigte Zugang zu Gesundheitsfürsorge und Gesundheitsbetreuung hervorgehoben werden. Die Staaten haben eine besondere Verpflichtung, jenen, die nicht über ausreichende Mittel verfügen, Krankenversicherung und Gesundheitsfürsorge zur Verfügung zu stellen und jegliche unerlaubte Diskriminierung bei der Gesundheitsfürsorge und den Gesundheitsdiensten zu verhindern, insbesondere im Hinblick auf die Kernverpflichtungen des Rechts auf Ge- 
sundheit. $^{15}$ Eine unangemessene Verteilung von Gesundheitsressourcen kann zu Diskriminierung führen, die nicht direkt zu erkennen ist. Zum Beispiel sollte nicht in unverhältnismäßig teure Gesundheitspflegedienste investiert werden, die oft nur einem kleinen, privilegierten Teil der Bevölkerung zugänglich sind, sondern vielmehr in die primäre und vorbeugende Gesundheitsfürsorge, die einem weitaus größeren Teil der Bevölkerung Vorteile bringt.

\section{Geschlechterperspektive}

20. Der Ausschuss empfiehlt, dass die Vertragsstaaten Geschlechterperspektiven in ihre gesundheitspolitischen Richtlinien, Planungen, Programme und in ihre Forschung aufnehmen, um eine bessere Gesundheit für Frauen wie für Männer zu erreichen. Ein geschlechtsspezifischer Ansatz erkennt an, dass biologische und soziokulturelle Faktoren die Gesundheit von Männern und Frauen stark beeinflussen. Die Aufschlüsselung gesundheitlicher, sozialer und wirtschaftlicher Daten im Hinblick auf das Geschlecht ist wesentlich, um Ungleichheiten bei der Gesundheit zu erkennen und $\mathrm{zu}$ beheben.

\section{Frauen und das Recht auf Gesundheit}

21. Um die Diskriminierung von Frauen zu beseitigen, besteht die Notwendigkeit, eine umfassende nationale Strategie für die Förderung des Rechts der Frau auf Gesundheit während ihres gesamten Lebens zu entwickeln und umzusetzen. Eine solche Strategie sollte sowohl Eingriffe, die der Vorbeugung und Behandlung von Krankheiten von Frauen dienen, als auch politische Maßnahmen beinhalten, um ihnen vollen Zugang zu hochwertiger und erschwinglicher Gesundheitsfürsorge zur Verfügung zu stellen. Hierzu zählen auch Beratungsdienste für die sexuelle und reproduktive Gesundheit. Ein wichtiges Ziel sollte in der Verminderung von Gesundheitsrisiken für Frauen liegen, insbesondere in der Senkung der Muttersterblichkeit und dem Schutz von Frauen vor häuslicher Gewalt. Die Verwirklichung des Rechts der Frau auf Gesundheit verlangt die Beseitigung aller Hindernisse, die den Zugang zu Gesundheitsdiensten, Bildung und Information beeinträchtigen, einschließlich im Hinblick auf sexuelle und reproduktive Ge-

$15 \mathrm{Zu}$ den Kernverpflichtungen vgl. Abs. 43 und 44 der vorliegenden Allgemeinen Bemerkung. 
sundheit. Außerdem ist es wichtig, vorbeugende, fördernde und heilende Maßnahmen zu ergreifen, um Frauen vor dem Einfluss schädlicher traditioneller kultureller Praktiken zu schützen, die ihre Fortpflanzungsrechte beeinträchtigen.

\section{Kinder und Jugendliche}

22. Artikel 12 Abs. 2 (a) umreißt die Notwendigkeit, Maßnahmen zu ergreifen, um die Kindersterblichkeit zu senken und die gesunde Entwicklung von Säuglingen und Kindern zu fördern. Internationale Menschenrechtsabkommen haben anerkannt, dass Kinder und Jugendliche das Recht haben, ein Höchstmaß an Gesundheit zu erreichen sowie Zugang zu Einrichtungen für die Behandlung von Krankheiten zu erhalten. ${ }^{16}$ Das Übereinkommen über die Rechte des Kindes weist die Staaten an, für das Kind und seine Familie den Zugang zu elementaren Gesundheitsdiensten einschließlich der prä- und postnatalen Betreuung für Mütter, zu gewährleisten. Das Übereinkommen verbindet diese Ziele damit, kindgemäße Informationen über präventives und gesundheitsförderndes Verhalten bereitzustellen und Familien und Gemeinschaften bei der Umsetzung dieser Praktiken zu unterstützen. Das Diskriminierungsverbot verlangt, dass Mädchen und Jungen einen gleichberechtigten Zugang zu angemessener Ernährung, einer sicheren Umwelt sowie zu physisch und psychologisch ausgerichteten Gesundheitsdiensten haben. Es besteht zudem die Notwendigkeit, effektive und angemessene Maßnahmen zu verabschieden, um schädliche traditionelle Praktiken zu beseitigen, die die Gesundheit von Kindern, insbesondere von Mädchen betreffen, einschließlich der Kinderehe, der weiblichen Genitalverstümmelung, der Bevorzugung von männlichen Kindern bei der Ernährung und Pflege. ${ }^{17}$ Kindern mit Behinderungen sollte die Chance gegeben werden, ein erfüllendes und ein Leben in Würde zu genießen und am Leben ihrer Gemeinschaft teilzuhaben.

23. Die Vertragsstaaten sollten für eine sichere und unterstützende Umwelt mit sozialem Rückhalt für Jugendliche sorgen, die Gelegenheit bietet, sich

16 Artikel 24 Abs. 1 des Übereinkommens über die Rechte des Kindes.

17 Vgl. Resolution der Weltgesundheitsversammlung WHA47.10, 1994, unter dem Titel »Gesundheit von Mutter und Kind und Familienplanung: Traditionelle Praktiken, die für die Gesundheit von Frauen und Kindern schädlich sind«. 
an gesundheitsbezogenen Entscheidungen zu beteiligen, Fertigkeiten für das Leben zu entwickeln, angemessene Information und Betreuung zu erhalten und ihre Entscheidungen bezüglich ihres Verhaltens im Hinblick auf Gesundheit mitbestimmen zu können. Die Verwirklichung des Rechts von Jugendlichen auf Gesundheit ist von der Entwicklung einer jugendfreundlichen Gesundheitsfürsorge abhängig, die die Vertraulichkeit und Privatsphäre achtet und die geeignete Betreuungsdienste für die sexuelle und reproduktive Gesundheit beinhaltet.

24. Bei allen politischen Maßnahmen und Programmen, die darauf abzielen, das Recht von Kindern und Jugendlichen auf Gesundheit zu gewährleisten, soll das Wohl der Kinder und Jugendlichen im Vordergrund stellen.

\section{Ältere Menschen}

25. Im Hinblick auf die Verwirklichung des Rechts älterer Menschen auf Gesundheit bestätigt der Ausschuss in Übereinstimmung mit den Absätzen 34 und 35 der Allgemeinen Bemerkung Nr. 6 (1995) die Bedeutung eines integrierten Ansatzes, der Elemente der Vorbeugung, der Rehabilitation und der Pflege miteinander verbindet. Derartige Maßnahmen sollten regelmäßige Untersuchungen für beide Geschlechter beinhalten; körperliche und psychologische Rehabilitationsmaßnahmen, die die Funktionsfähigkeit und Selbstständigkeit älterer Menschen aufrecht erhalten sollen; und Hingabe und Pflege für chronisch Kranke und sterbenskranke Menschen, indem ihnen vermeidbarer Schmerz erspart und ihnen ermöglicht wird, in Würde zu sterben.

\section{Menschen mit Behinderungen}

26. Der Ausschuss bekräftigt Absatz 34 seiner Allgemeinen Bemerkung Nr. 5, der sich dem Problem von Menschen mit Behinderungen im Zusammenhang mit dem Recht auf körperliche und geistige Gesundheit zuwendet. Überdies betont der Ausschuss die Notwendigkeit, sicherzustellen, dass nicht nur der öffentliche Gesundheitssektor, sondern auch private Anbieter von Gesundheitsdiensten und Einrichtungen den Grundsatz des Diskriminierungsverbots in Bezug auf Menschen mit Behinderungen befolgen. 


\section{Indigene Völker}

27. Mit Rücksicht auf das sich entwickelnde Völkerrecht, die allgemeine Praxis der Staaten und die jüngsten Maßnahmen, die von Staaten in Bezug auf indigene Völker ergriffen wurden, ${ }^{18}$ empfiehlt der Ausschuss, jene Elemente zu bestimmen, die das Recht indigener Völker auf Gesundheit definieren, um Staaten mit indigenen Völker bessere Möglichkeiten zu vermitteln, die Vorschriften des Artikel 12 umzusetzen. Der Ausschuss ist der Ansicht, dass indigene Völker das Recht auf Sondermaßnahmen haben, um ihren Zugang zu Gesundheits- und Pflegediensten zu verbessern. Die Gesundheitsdienste sollten kulturell angemessen sein, indem sie traditionelle präventive Pflege, Heilpraktiken und traditionelle Medizin nutzen. Die Staaten sollten für indigene Völker Ressourcen zur Verfügung stellen, um entsprechende Einrichtungen zu planen, zu schaffen und zu kontrollieren, so dass diese Völker das für jede/n erreichbare Höchstmaß an körperlicher und geistiger Gesundheit genießen können. Ebenso sollten lebensnotwendige medizinische Pflanzen, Tiere und Mineralien, die indigene Völker für ihre Gesundheit benötigen, geschützt werden. Der Ausschuss weist darauf

18 Kürzlich vereinbarte internationale Normen, die für indigene Völker relevant sind, beinhalten: das ILO-Übereinkommen Nr. 169 über eingeborene und in Stämmen lebende Völker in unabhängigen Ländern (1989); Artikel 29 (c) und (d) des Übereinkommens über die Rechte des Kindes (1989); Artikel 8 (j) des Übereinkommens über biologische Vielfalt (1992), der die Empfehlung ausspricht, dass Staaten die Kenntnisse, Innovationen und Praktiken indigener Gemeinschaften achten, bewahren und aufrecht erhalten sollen; Agenda 21der Konferenz der Vereinten Nationen über Umwelt und Entwicklung (1992), insbesondere Kapitel 26; und Teil I, Abs. 20 der Wiener Erklärung und des Aktionsprogramms, das feststellt, dass Staaten konzertierte positive Schritte unternehmen sollten, um die Achtung der Menschenrechte für alle indigenen Völker auf der Grundlage des Diskriminierungsverbots zu gewährleisten. Vgl. auch die Präambel und Artikel 3 des Rahmenübereinkommens über Klimawandel der Vereinten Nationen (1992) und Artikel 10 Abs. 2 (e) des Übereinkommens der Vereinten Nationen zur Bekämpfung der Wüstenbildung in den von Dürre und/oder Wüstenbildung schwer betroffenen Ländern, insbesondere in Afrika (1994). Während der vergangenen Jahre hat eine wachsende Anzahl von Staaten ihre Verfassung geändert und Gesetzgebung eingeführt, die spezifische Rechte der indigenen Völker anerkennt. 
hin, dass bei den Gemeinschaften indigener Völker die Gesundheit des Einzelnen oft mit der Gesundheit der Gemeinschaft insgesamt verknüpft ist und somit eine kollektive Dimension besitzt. In dieser Hinsicht ist der Ausschuss der Ansicht, dass Entwicklungen, die zur Vertreibung indigener Völker aus ihren angestammten Gebieten und ihrer traditionellen Umgebung führen und bei denen ihre Nahrungsgrundlagen entzogen und ihre symbiotische Beziehung mit ihrem Land zerstört, einen schädlichen Effekt auf ihre Gesundheit haben.

\section{Beschränkungen}

28. Manchmal werden Probleme mit der öffentlichen Gesundheit von Staaten als Rechtfertigung für die Einschränkung anderer Grundrechte herangezogen. Der Ausschuss möchte betonen, dass die Schrankenklausel des Pakts, der Artikel 4, vorrangig die Rechte von Individuen schützen soll, anstatt Staaten zu gestatten, Beschränkungen einzuführen. Infolgedessen ist zum Beispiel ein Vertragsstaat, der aus Gründen der öffentlichen Sicherheit und Ordnung die Freizügigkeit (i. S. v. Bewegungsfreiheit) von Menschen mit ansteckenden Krankheiten, wie HIV/AIDS, einschränkt oder der diese Menschen sogar einweist, der sich weigert, Ärzte für jene Menschen zuzulassen, die für Regierungsgegner gehalten werden, oder der darin versagt, der Gemeinschaft Immunisierungsmittel gegen gravierende Infektionskrankheiten zur Verfügung zu stellen, verpflichtet, solche weitreichenden Maßnahmen gemäß den einzelnen Bestimmungen des Artikel 4 rechtfertigen. Solche Einschränkungen müssen gesetzlich vorgesehen sein, internationalen Menschenrechtsstandards entsprechen, mit dem Wesen der Paktrechte vereinbar sein und dürfen ausschließlich dem Zweck dienen, das allgemeine Wohl in einer demokratischen Gesellschaft zu fördern.

29. Gemäß Artikel 5 Abs. 1 müssen derartige Beschränkungen verhältnismäßig sein, das heißt, es muss das am wenigsten einschränkende Mittel angewendet werden, wenn mehrere Arten von Einschränkungen verfügbar sind. Sogar in Fällen, in denen Einschränkungen auf Grund des Schutzes der öffentlichen Gesundheit erlaubt sind, sollten sie von begrenzter Dauer sein und einer Überprüfung unterworfen werden. 


\section{Die Verpflichtungen DeR VertragsstaAten}

\section{Allgemeine rechtliche Verpflichtungen}

30. Obgleich der Pakt eine schrittweise Verwirklichung vorsieht und die Zwänge anerkennt, die auf begrenzt verfügbare Ressourcen zurückzuführen sind, erlegt er den Staaten auch verschiedene Verpflichtungen auf, die eine sofortige Wirkung haben. Die Vertragsstaaten haben unmittelbare Verpflichtungen in Bezug auf das Recht auf Gesundheit, wie zum Beispiel die Verpflichtung, die Ausübung dieses Recht ohne jegliche Diskriminierung (Artikel 2 Abs. 2) zu gewährleisten, und die Verpflichtung, Maßnahmen (Artikel 2 Abs. 1) zur vollen Verwirklichung des Artikel 12 zu treffen. Diese Maßnahmen müssen wohl überlegt und konkret sein und die volle Verwirklichung des Rechts auf Gesundheit zum Ziel haben. ${ }^{19}$

31. Die schrittweise Verwirklichung des Rechts auf Gesundheit über einen gewissen Zeitraum sollte nicht so interpretiert werden, als würde sie den Verpflichtungen der Vertragsstaaten jegliche Bedeutung entziehen. Vielmehr bedeutet schrittweise Verwirklichung, dass Staaten die spezifische und kontinuierliche Verpflichtung haben, so schnell und effektiv wie möglich die volle Verwirklichung des Artikels 12 zu erreichen. ${ }^{20}$

32. Wie bei allen anderen Rechten des Pakts besteht eine überwiegende Annahme, dass rückläufige Maßnahmen in Bezug auf das Recht aus Gesundheit unerlaubt sind. Sollten rückläufige Maßnahmen absichtlich ergriffen werden, dann obliegt dem Vertragsstaat die Beweislast, dass diese Maßnahmen nach sorgfältigster Abwägung aller Alternativen eingeführt wurden, dass alle verfügbaren Ressourcen ausgeschöpft wurden und die Maßnahmen im Hinblick auf die Gesamtheit der Paktrechte gerechtfertigt sind. $^{21}$

19 Vgl. Allgemeine Bemerkung Nr. 13, Abs. 43.

20 Vgl. Allgemeine Bemerkung Nr. 3, Abs. 9; Allgemeine Bemerkung Nr. 13, Abs. 44.

21 Vgl. Allgemeine Bemerkung Nr. 3, Abs. 9; Allgemeine Bemerkung Nr. 13, Abs. 44. 
33. Wie alle Menschenrechte erlegt das Recht auf Gesundheit den Vertragsstaaten drei Arten oder Ebenen von Verpflichtungen auf: Eine Achtungspflicht, eine Schutzpflicht und eine Gewährleistungspflicht. Dabei enthält die Gewährleistungspflicht die Verpflichtung zu ermöglichen, zur Verfügung zu stellen und zu fördern. Die Achtungspflicht verlangt von den Staaten, von einem unmittelbaren oder mittelbaren Eingriff in den Genuss des Rechts auf Gesundheit Abstand zu nehmen. Die Schutzpflicht verlangt von den Staaten, Maßnahmen zu ergreifen, die Dritte daran hindern, die Garantien des Artikel 12 zu beeinträchtigen. Schließlich verlangt die Gewährleistungspflicht von den Staaten, angemessene legislative, administrative, finanzielle, gerichtliche, fördernde und sonstige Maßnahmen zu verabschieden, die die volle Verwirklichung des Rechts auf Gesundheit zum Ziel haben.

\section{Besondere rechtliche Verpflichtungen}

34. Insbesondere unterliegen die Staaten der Verpflichtung, das Recht auf Gesundheit zu achten, indem sie es zum Beispiel unterlassen, den gleichberechtigten Zugang zu vorbeugenden, heilenden und lindernden Gesundheitsdiensten für jeden Menschen zu verweigern oder zu beschränken, einschließlich für Gefangene oder Inhaftierte, Minderheiten, Asylsuchende und illegale Immigranten; indem sie darauf verzichten, diskriminierende Praktiken als politische Maßnahme durchzusetzen, und es unterlassen, diskriminierende Praktiken im Hinblick auf den Gesundheitsstatus und die Bedürfnisse von Frauen einzuführen. Weiterhin verlangt die Achtungspflicht, von dem Verbot oder der Behinderung traditioneller präventiver Pflege, Heilpraktiken und traditioneller Medizin sowie von der Verbreitung unsicherer Medikamente und von erzwungenen medizinischen Behandlungen Abstand zu nehmen, es sei denn, es besteht eine Ausnahmesituation für die Behandlung psychischer Krankheit oder für die Vorbeugung und Bekämpfung übertragbarer Krankheiten. Solche Ausnahmefälle sollten besonders restriktiven Bedingungen unterliegen und best practices sowie internationale Standards beachten, einschließlich der »Grundsätze für den Schutz von psychisch Kranken und die Verbesserung der psychiatrischen Versorgung. «22 Zusätzlich sollten die Staaten es unterlassen, den Zugang zu Verhütungsmitteln und zu sonstigen für die Aufrechterhaltung der sexuellen

22 Resolution der Generalversammlung 46/119 (1991). 
und reproduktiven Gesundheit erforderlichen Mitteln zu beschränken; gesundheitsbezogene Information zu zensieren, einzubehalten oder absichtlich missverständlich darzustellen, einschließlich Information zur Sexualerziehung, sowie die Beteiligung der Menschen an Gesundheitsthemen zu behindern. Die Staaten sollten es auch unterlassen, Luft, Wasser und Erde unrechtmäßig zu verschmutzen, etwa durch industriellen Abfall von staatlichen Einrichtungen sowie durch den Einsatz oder den Test nuklearer, biologischer oder chemischer Waffen, wenn derartige Verfahren zur Freisetzung von Substanzen führen, die der menschlichen Gesundheit schaden, und wenn sie den Zugang zu Gesundheitsdiensten als Repressalie einschränken, zum Beispiel während bewaffneter Konflikte unter Verletzung des humanitären Völkerrechts.

35. Die Schutzpflicht beinhaltet unter anderem die Pflicht des Staates, Gesetze zu verabschieden oder andere Maßnahmen zu ergreifen, die den gleichberechtigten Zugang zu Gesundheitsfürsorge und zu Gesundheitsdiensten gewährleisten, die von Dritten bereitgestellt werden, und sicherzustellen, dass die Privatisierung des Gesundheitssektors keine Bedrohung im Hinblick auf die Verfügbarkeit, Zugänglichkeit, Annehmbarkeit und Qualität medizinischer Einrichtungen und ärztlicher Betreuung nach sich zieht; die Vermarktung medizinischer Einrichtungen und von Arzneien durch Dritte zu kontrollieren; und zu gewährleisten, dass praktische Ärzte und andere im Gesundheitssektor Beschäftigte entsprechende Ausbildung und Fähigkeiten besitzen und den ethischen Verhaltenskodex befolgen. Die Staaten sind außerdem verpflichtet sicherzustellen, dass schädliche soziale oder traditionelle Praktiken den Zugang zu prä- und postnataler Betreuung und Familienplanung nicht beeinträchtigen; Dritte daran zu hindern, Frauen zu traditionellen Praktiken zu zwingen, zum Beispiel zur weiblichen Genitalverstümmelung; und Maßnahmen zu ergreifen, um alle schutzbedürftigen oder an den Rand gedrängten Gruppen der Gesellschaft, insbesondere Frauen, Kinder, Jugendliche und ältere Menschen, vor geschlechtsbezogener Gewalt zu schützen. Die Vertragsstaaten sollten auch sicherstellen, dass Dritte den Zugang zu Gesundheitsinformation und Gesundheitsdiensten nicht einschränken.

36. Die Gewährleistungspflicht verlangt von den Vertragsstaaten, das Recht auf Gesundheit auf nationaler politischer und gesetzlicher Ebene, vorzugs- 
weise auf dem Gesetzgebungsweg, angemessen anzuerkennen und eine nationale Gesundheitspolitik in detaillierter Ausgestaltung anzunehmen, um das Recht auf Gesundheit zu verwirklichen. Die Staaten müssen das Vorhandensein von Gesundheitsfürsorge einschließlich von Immunisierungsprogrammen gegen schwere Infektionskrankheiten gewährleisten sowie garantieren, dass für jeden Menschen gleichberechtigter Zugang zu den elementaren Voraussetzungen von Gesundheit, zum Beispiel zu nährstoffreicher sicherer Nahrung und Trinkwasser, zu Sanitäranlagen, angemessener Unterkunft und angemessenen Lebensbedingungen vorhanden ist. Die öffentliche Gesundheitsinfrastruktur sollte Beratungsdienste für die sexuelle und reproduktive Gesundheit zur Verfügung stellen, einschließlich einer Mutterschaftsbetreuung, insbesondere in ländlichen Gebieten. Die Staaten müssen die angemessene Ausbildung von Ärzten/innen und sonstigem medizinischen Personal, die Versorgung mit einer hinreichenden Anzahl von Krankenhäusern, Kliniken und anderen Gesundheitseinrichtungen gewährleisten sowie Einrichtungen für Beratung und psychologische Gesundheitsfürsorge fördern und unterstützen. Dabei sollte die geographische Verteilung dieser Einrichtungen angemessen berücksichtigt werden. Weitere Verpflichtungen beinhalten die Versorgung mit einem öffentlichen, privaten oder gemischten Krankenversicherungssystem, das für jeden Menschen erschwinglich ist, die Förderung von medizinischer Forschung und Gesundheitserziehung ebenso wie Informationskampagnen insbesondere im Hinblick auf HIV/AIDS, sexuelle und reproduktive Gesundheit, im Blick auf traditionelle Praktiken, häusliche Gewalt, Missbrauch von Alkohol und Konsum von Tabak, Drogen und anderen schädlichen Substanzen. Die Staaten sind auch aufgefordert, Maßnahmen gegen Umwelt- und berufliche Gesundheitsrisiken und gegen jegliche Bedrohungen, die in epidemiologischen Studien aufgezeigt werden, zu treffen. Zu diesem Zweck sollten sie nationale politische Richtlinien formulieren und umsetzen, die darauf abzielen, die Verschmutzung von Luft, Wasser und Erde zu reduzieren, einschließlich der Verschmutzung durch Schwermetalle wie Blei, das aus Benzin entweicht, zu reduzieren. Weiterhin sind die Vertragsstaaten aufgefordert, eine in sich kohärente nationale Politik zu formulieren, umzusetzen und in regelmäßigen Abständen zu überprüfen, um das Risiko von Berufsunfällen und Berufskrankheiten zu minimieren. Dies gilt ebenso für eine 
kohärente nationale Politik zu beruflicher Sicherheit und Gesundheitsdiensten. $^{23}$

37. Die Gewährleistungspflicht fordert von den Staaten unter anderem, Positivmaßnahmen zu ergreifen, die es Individuen und Gemeinschaften ermöglichen, das Recht auf Gesundheit zu genießen. Die Vertragsstaaten sind auch verpflichtet, ein spezifisches Paktrecht zu gewährleisten, wenn Individuen oder Gruppen aus Gründen, die sich ihrer Kontrolle entziehen, unfähig sind, das betreffende Recht mit eigenen Mitteln zu verwirklichen. Die Verpflichtung, das Recht auf Gesundheit zu gewährleisten (fördern) verlangt von den Vertragsstaaten, Maßnahmen zu ergreifen, die die Gesundheit der Bevölkerung fördern, aufrechterhalten und wiederherstellen. Solche Verpflichtungen beinhalten:

(i) das Erkennen von Faktoren, die die Gesundheit begünstigen und fördern, zum Beispiel durch Forschung und Verbreitung von Informationen;

(ii) kulturell angemessene Gesundheitsdienste und ausgebildetes Personal, das die besonderen Bedürfnisse schutzbedürftiger und ausgegrenzter Gruppen erkennen und entsprechend darauf reagieren kann;

23 Elemente einer solchen Politik sind die Identifizierung, Bestimmung, Genehmigung und Kontrolle gefährlicher Materialien, Ausrüstungen, Substanzen, Wirkstoffe und Arbeitsprozesse; die Versorgung der Arbeiter/innen mit Gesundheitsinformationen und, falls nötig, mit geeigneter Schutzkleidung und Ausrüstung; die Durchsetzung von Gesetzen und Verordnungen durch angemessene Inspektion; die Pflicht, über Berufsunfälle und -krankheiten Mitteilung zu machen, die Durchführung von Untersuchungen und jährlichen Statistiken; der Schutz der Arbeiter/innen und von deren Vertreter/innen vor Disziplinarmaßnahmen für Handlungen, die sie in Übereinstimmung mit einer solchen Politik unternommen haben; und die Versorgung mit betriebsärztlichen Diensten, die wesentlich vorbeugende Aufgaben wahrnehmen. Vgl. ILO-Übereinkommen über den Arbeitsschutz, 1981 (Nr.155), und das Übereinkommen über die betriebsärztlichen Dienste, 1985 (Nr. 161). 
(iii) die Gewährleistung, dass ein Staat seinen Verpflichtungen zur Verbreitung von geeigneten Informationen nachkommt, die über gesunde Lebensführung und Ernährung, schädliche traditionelle Praktiken und die Verfügbarkeit von entsprechenden Leistungen aufklärt;

(iv) Menschen darin zu unterstützen, wohl überlegte Entscheidungen hinsichtlich ihrer Gesundheit zu treffen.

\section{Internationale Verpflichtungen}

38. In seiner Allgemeinen Bemerkung Nr. 3 richtete der Ausschuss die Aufmerksamkeit auf die Verpflichtung aller Vertragsstaaten, einzeln sowie durch internationale Hilfe und Zusammenarbeit, insbesondere wirtschaftlicher und technischer Art, Maßnahmen zu treffen, um nach und nach die vollständige Verwirklichung der Paktrechte, wie des Rechts auf Gesundheit, zu erreichen. Im Geiste von Artikel 56 der Charta der Vereinten Nationen, besonderer Vorschriften des Pakts (Artikel 12, 2 Abs. 1, 22 und 23) und der Erklärung von Alma-Ata über primäre Gesundheitsversorgung sollten die Vertragsstaaten die wesentliche Rolle internationaler Zusammenarbeit anerkennen und gemeinsam und einzeln Maßnahmen ergreifen, um die vollständige Verwirklichung des Rechts auf Gesundheit zu erreichen. In dieser Hinsicht werden die Staaten auf die Erklärung von Alma-Ata verwiesen, die zum Ausdruck bringt, dass die bestehende große Ungleichheit im Gesundheitsstatus der Menschen, insbesondere zwischen Menschen in den entwickelten Länder und denen in den Entwicklungsländern, aber auch innerhalb eines Staates, politisch, sozial und wirtschaftlich nicht akzeptabel und ihre Überwindung deshalb ein gemeinsames Anliegen aller Staaten ist. $^{24}$

39. Um ihren internationalen Verpflichtungen aus Artikel 12 nachzukommen, müssen Vertragsstaaten das Recht auf Gesundheit in anderen Ländern achten und Dritte daran hindern, dieses Recht in anderen Ländern zu verletzen, falls es möglich ist, diese mit rechtlichen oder politischen Mitteln, in Übereinstimmung mit der Charta der Vereinten Nationen und einschlägigem Völkerrecht, zu beeinflussen. Je nach Verfügbarkeit von Ressourcen

24 Artikel II, Erklärung von Alma-Ata, Bericht der Internationalen Konferenz über primäre Gesundheitsversorgung, Alma-Ata, 6. bis 12. September 1978, in: World Health Organization, Health for All Series, Nr.1, WHO, Genf, 1978. 
sollten die Staaten den Zugang zu elementaren medizinischen Einrichtungen und ärztlicher Betreuung in anderen Ländern, wann immer möglich, erleichtern und auf Anfrage notwendige Hilfe zur Verfügung stellen. ${ }^{25}$ Die Vertragsstaaten sollten sicherstellen, dass dem Recht auf Gesundheit in internationalen Abkommen gebührende Aufmerksamkeit geschenkt wird, und sollten dazu die Entwicklung weiterer Rechtsinstrumente in Erwägung ziehen. Im Hinblick auf den Abschluss anderer internationaler Abkommen sollten die Staaten Maßnahmen ergreifen, um zu gewährleisten, dass das Recht auf Gesundheit davon nicht negativ beeinflusst wird. In ähnlicher Weise haben die Vertragsstaaten die Verpflichtung zu gewährleisten, in ihren Handlungen als Mitglieder internationaler Organisationen das Recht auf Gesundheit gebührend zu berücksichtigen. Entsprechend sollten jene Vertragsstaaten, die Mitglieder internationaler Finanzeinrichtungen, insbesondere des Internationalen Währungsfonds, der Weltbank und regionaler Entwicklungsbanken sind, dem Schutz des Rechts auf Gesundheit bei der Einflussnahme auf die Finanzpolitik, die Kreditvereinbarungen und die internationalen Maßnahmen dieser Institutionen größere Aufmerksamkeit schenken.

40. Die Vertragsstaaten haben eine gemeinsame und eine individuelle Verantwortung, in Übereinstimmung mit der Charta der Vereinten Nationen und entsprechenden Resolutionen der UN-Generalversammlung und der Weltgesundheitsversammlung (World Health Assembly) bei der Katastrophenhilfe und der humanitären Hilfe in Zeiten des Notstands zusammenzuarbeiten, einschließlich bei der Hilfe für Flüchtlinge und Binnenvertriebene. Jeder Staat sollte zur Lösung solcher Aufgabe unter Ausschöpfung seiner Möglichkeiten beitragen. Bei der Versorgung mit internationaler medizinischer Hilfe, der Verteilung und Verwaltung der Hilfsgüter, darunter von sicherem Trinkwasser, Nahrungsmitteln und Arzneimitteln, und bei der finanziellen Hilfe sollten die am stärksten schutzbedürftigen oder ausgegrenzten Gruppen der Bevölkerung vorrangig bedacht werden. Überdies hat die internationale Gemeinschaft im Hinblick auf die grenzüberschreitende Verbreitung einiger übertragbarer Krankheiten eine kollektive Verantwortung, sich dieses Problems anzunehmen. Die wirtschaftlich entwickelten

25 Vgl. Abs. 45 dieser Allgemeinen Bemerkung. 
Staaten haben eine besondere Verantwortung und ein ebensolches Interesse daran, den ärmeren Entwicklungsländern in dieser Hinsicht zu helfen.

41. Die Vertragsstaaten sollten es zu allen Zeiten unterlassen, Embargos oder ähnliche Maßnahmen zu ergreifen, die die Versorgung eines anderen Staates mit geeigneten Medikamenten und medizinischer Ausstattung beschränken. Einfuhrbeschränkungen auf solche Güter sollten niemals als Instrument politischen und wirtschaftlichen Drucks benutzt werden. In dieser Hinsicht ruft der Ausschuss seine Position zum Zusammenhang von wirtschaftlichen Sanktionen und der Achtung der wirtschaftlichen, sozialen und kulturellen Rechte in Erinnerung, die in der Allgemeinen Bemerkung Nr. 8 zum Ausdruck gebracht wird.

42. Obgleich nur Staaten Parteien des Pakts sind und daher letztendlich nur sie für dessen Nichteinhaltung zur Rechenschaft gezogen werden können, haben alle Mitglieder der Gesellschaft - Einzelpersonen, wie Mediziner, ebenso wie Familien, lokale Gemeinschaften, zwischenstaatliche und nichtstaatliche Organisationen, Organisationen der Zivilgesellschaft und die Privatwirtschaft - gewisse Verantwortlichkeiten im Hinblick auf die Verwirklichung des Rechts auf Gesundheit. Die Vertragsstaaten sollten deshalb für ein Umfeld sorgen, das es erleichtert, diesen Verantwortlichkeiten nachzukommen.

\section{Kernverpflichtungen}

43. In seiner Allgemeinen Bemerkung Nr. 3 bestätigt der Ausschuss, dass die Vertragsstaaten eine Kernverpflichtung haben, wenigstens einen Mindeststandard eines jeden Paktrechts zu gewährleisten, einschließlich einer grundlegenden primären Gesundheitsversorgung. In Verbindung mit aktuelleren Instrumenten, wie dem Aktionsprogramm der Internationalen Konferenz über Bevölkerung und Entwicklung, ${ }^{26}$ liefert die Erklärung von Alma-Ata eine verbindliche Leitlinie für die Kernverpflichtungen, die aus Artikel 12 erwachsen. Entsprechend sind nach Ansicht des Ausschusses zumindest folgende Pflichten in den Kernverpflichtungen enthalten:

26 Bericht der Internationalen Konferenz über Bevölkerung und Entwicklung, Kairo, 5.-13. September 1994 (Publikationen der Vereinten Nationen, Sales Nr. E.95.XIII.18), Kap. I, Resolution 1, Anhang, Kap. VII und VIII. 
(a) das Recht auf Zugang zu medizinischen Einrichtungen und ärztlicher Betreuung ohne jegliche Diskriminierung, insbesondere im Hinblick auf schutzbedürftige und ausgegrenzte Gruppen, zu gewährleisten;

(b) den Zugang zu einem Mindestmaß an elementaren Nahrungsmitteln, die ausgewogen und sicher sind, zu gewährleisten, um für jedermann ein Leben ohne Hunger zu garantieren;

(c) den Zugang zu Unterkunft und Sanitäranlagen sowie eine adäquate Versorgung mit sicherem Trinkwasser zu gewährleisten;

(d) elementare Arzneimittel zur Verfügung zu stellen, die in regelmäßigen Abständen durch das Aktionsprogramm über unentbehrliche Arzneimittel der WHO definiert werden;

(e) eine gerechte Verteilung aller medizinischer Einrichtungen und ärztlicher Betreuung sicherzustellen;

(f) eine nationale Gesundheitsstrategie und einen Aktionsplan zu verabschieden und umzusetzen, der sich auf eine epidemiologische Grundlage stützt und die Gesundheitsbelange der gesamten Bevölkerung einbezieht; die Strategie und der Aktionsplan sollen auf der Grundlage eines partizipatorischen und transparenten Prozesses entworfen und in regelmäßigen Abständen überprüft werden; sie sollen Methoden beinhalten, wie etwa Indikatoren und Benchmarks, mit denen der Fortschritt bei der Verwirklichung des Rechts auf Gesundheit genau beobachtet werden kann; der Prozess für den Entwurf der Strategie und des Aktionsplans soll ebenso wie deren Inhalt allen schutzbedürftigen oder ausgegrenzten Gruppen besondere Aufmerksamkeit widmen.

44. Der Ausschuss bestätigt außerdem, dass die folgenden Verpflichtungen von vergleichbarer Priorität sind:

(a) Gesundheitsfürsorge im Hinblick auf reproduktive Gesundheit, Mutterschaft (pränatal und postnatal) und das Kind; 
(b) Immunisierung gegen verbreitete Infektionskrankheiten, die in der Gemeinschaft auftreten;

(c) Maßnahmen zur Verhinderung, Behandlung und Bekämpfung epidemischer und endemischer Krankheiten;

(d) Aufklärung und Information über die wesentlichen Gesundheitsprobleme der Gemeinschaft, einschließlich über Möglichkeiten ihrer Verhinderung und Bekämpfung;

(e) angemessene Ausbildung des Personals im Gesundheitswesen, die auch Kenntnisse zum Verhältnis von Gesundheit und Menschenrechten vermittelt.

45. Um jeglichem Zweifel vorzubeugen, möchte der Ausschuss betonen, dass es für die Vertragsstaaten und andere Akteure, die in der Lage sind zu helfen, besonders notwendig ist, »internationale Hilfe und Zusammenarbeit, insbesondere wirtschaftlicher und technischer Art «, ${ }^{27}$ zur Verfügung zu stellen, damit Entwicklungsländer ihre Kern- und sonstigen Verpflichtungen erfüllen können, wie diese oben in den Absätzen 43 und 44 beschrieben sind.

\section{Verletzungen}

46. Wenn der normative Inhalt des Artikel 12 (Teil I) auf die staatlichen Verpflichtungen (Teil II) angewendet wird, dann wird ein dynamischer Prozess in Gang gesetzt, der die Feststellung von Verletzungen des Rechts auf Gesundheit erleichtert. Die folgenden Absätze geben Beispiele für Verletzungen des Artikels 12.

47. Bei der Feststellung, welche Handlung oder Unterlassung auf eine Verletzung des Rechts auf Gesundheit hinauslaufen, ist es wichtig, zwischen der Unfähigkeit eines Vertragsstaates, seine Verpflichtungen gemäß Artikel 12 einzuhalten, und dem Unwillen dazu zu unterscheiden. Dies folgt aus 
Artikel 12 Abs. 1, der von einem erreichbaren Höchstmaß an Gesundheit spricht, ebenso wie aus Artikel 2 Abs. 1 des Pakts, der jeden Vertragsstaat verpflichtet, die notwendigen Maßnahmen unter Ausschöpfung aller seiner Möglichkeiten zu treffen. Ein Staat, der nicht willens ist, seine verfügbaren Ressourcen für die Verwirklichung des Rechts auf Gesundheit auszuschöpfen, verletzt seine Verpflichtungen gemäß Artikel 12. Falls Ressourcenknappheit es einem Staat unmöglich macht, seinen Paktverpflichtungen vollständig nachzukommen, dann obliegt es ihm nachzuweisen, dass dennoch jede Anstrengung unternommen wurde, alle verfügbaren Mittel zu verwenden, um die oben beschriebenen Verpflichtungen vorrangig zu erfüllen. Es sollte jedoch betont werden, dass ein Vertragsstaat unter keinen, wie auch immer gearteten, Umständen seine Nichteinhaltung der oben in Absatz 43 dargestellten Kernverpflichtungen rechtfertigen kann, da diese nicht außer Kraft gesetzt werden können.

48. Verletzungen des Rechts auf Gesundheit können durch unmittelbare Handlungen des Staates oder durch sonstige Organe entstehen, die vom Staat unzureichend reguliert werden. Die Verabschiedung jeglicher rückläufiger Maßnahmen, die mit den Kernverpflichtungen des Rechts auf Gesundheit, wie oben in Absatz 43 beschrieben, unvereinbar sind, stellen eine Verletzung des Rechts auf Gesundheit dar. Eine Verletzungen durch positives Tun kann etwa durch die formelle Aufhebung oder Suspendierung von Gesetzgebung entstehen, die für die dauerhafte Inanspruchnahme des Rechts auf Gesundheit notwendig ist, oder die Verabschiedung von Gesetzen oder politischen Richtlinien, die offenkundig mit bereits bestehenden nationalen oder internationalen rechtlichen Verpflichtungen in Bezug auf das Recht auf Gesundheit unvereinbar sind.

49. Verletzungen des Rechts auf Gesundheit können auch durch die Unterlassung oder das Versäumnis von Staaten entstehen, jene notwendigen Maßnahmen zu ergreifen, die sich aus ihren rechtlichen Verpflichtungen ableiten. Zu Verletzungen durch Unterlassung gehören das Versäumnis, geeignete Maßnahmen zu treffen, die zur vollständigen Verwirklichung des Rechts eines jeden Menschen führen, ein Höchstmaß an körperlicher und geistiger Gesundheit zu erreichen, sowie das Versäumnis, über eine nationale Gesundheitspolitik zu Sicherheit und Gesundheit am Arbeitsplatz oder 
über betriebsärztliche Dienste zu verfügen, und das Versäumnis, einschlägige Gesetze durchzusetzen.

\section{Verletzungen der Achtungspflicht}

50. Verletzungen der Achtungspflicht umfassen solche staatlichen Handlungen, politischen Richtlinien oder Gesetze, die den in Artikel 12 des Pakts aufgestellten Normen zuwiderlaufen und die zu körperlichen Schäden, unnötigen Erkrankungen und vermeidbarer Sterblichkeit führen können. Beispiele sind die Verweigerung des Zugangs zu medizinischen Einrichtungen und ärztlicher Betreuung als Resultat von de jure oder de facto Diskriminierung von bestimmten Individuen oder Gruppen; die absichtliche Zurückhaltung oder Falschdarstellung von Informationen, die für den Schutz der Gesundheit oder eine Behandlung lebenswichtig sind; die Suspendierung von Gesetzgebung oder die Verabschiedung von Gesetzen oder politischen Richtlinien, wenn dadurch der Genuss eines Bestandteils des Rechts auf Gesundheit beeinträchtigt wird; und das Versäumnis des Staates, seine rechtlichen Verpflichtungen im Hinblick auf das Recht auf Gesundheit zu berücksichtigen, wenn bilaterale oder multilaterale Verträge mit anderen Staaten, internationalen oder sonstigen Organisationen sowie mit multinationalen Unternehmen eingegangen werden.

\section{Verletzungen der Schutzpflicht}

51. Verletzungen der Schutzpflicht entstehen aus dem Versäumnis eines Staates, alle notwendigen Maßnahmen zu ergreifen, um Menschen innerhalb seines Hoheitsgebiets vor Verletzungen des Rechts auf Gesundheit durch Dritte zu schützen. Diese Kategorie beinhaltet solche Unterlassungen wie das Versäumnis, Aktivitäten von Individuen, Gruppen oder Unternehmen so zu regulieren, dass eine Verletzung des Rechts auf Gesundheit anderer verhindert wird; das Versäumnis, Konsumenten und Arbeiter vor gesundheitsschädlichen Praktiken, zum Beispiel durch Arbeitgeber und Hersteller von Arzneimitteln oder Nahrungsmitteln, zu schützen; das Versäumnis, von der Herstellung, der Vermarktung und dem Konsum von Tabak, Narkotika und anderen schädlichen Substanzen abzuraten; das Versäumnis, Frauen vor Gewalt zu schützen oder Straftäter zu verfolgen; das Versäumnis, fortgesetzten schädlichen traditionellen medizinischen oder kulturellen Praktiken entgegenzutreten; und das Versäumnis, Gesetze zu erlassen oder 
durchzusetzen, um die Verschmutzung von Wasser, Luft und Erde durch fördernde und herstellende Industrien zu verhindern.

\section{Verletzungen der Gewährleistungspflicht}

52. Verletzungen der Gewährleistungspflicht entstehen durch das Versäumnis der Vertragsstaaten, alle notwendigen Maßnahmen zu treffen, um die Verwirklichung des Rechts auf Gesundheit zu gewährleisten. Als Beispiele lassen sich anführen: das Versäumnis, eine nationale Gesundheitspolitik zu beschließen oder umzusetzen, die darauf zugeschnitten ist, das Recht auf Gesundheit für jeden Menschen zu gewährleisten; unzureichende Aufwendungen oder eine fehlerhafte Aufteilung öffentlicher Mittel, die dazu führen, dass Individuen oder Gruppen, insbesondere schutzbedürftige oder ausgegrenzte Gruppen, das Recht auf Gesundheit nicht genießen können; das Versäumnis, die Verwirklichung des Rechts auf Gesundheit auf innerstaatlicher Ebene zu beobachten, zum Beispiel durch Indikatoren und Benchmarks zur Bestimmung des Rechts auf Gesundheit; das Versäumnis, Maßnahmen zu ergreifen, um die ungerechte Verteilung von medizinischen Einrichtungen und ärztlicher Betreuung zu vermindern; das Versäumnis, einen geschlechtsbezogenen Ansatz im Hinblick auf das Recht auf Gesundheit anzuwenden und die Säuglings- und Müttersterblichkeit zu senken. 


\section{UMSETZUNG AUF INNERSTAATLICHER EBENE}

\section{Rahmengesetzgebung}

53. Die am besten geeigneten Mittel und Wege zur Verwirklichung des Rechts auf Gesundheit werden sich zwangsläufig von einem Staat zum anderen erheblich unterscheiden. Jeder Staat besitzt einen Ermessensspielraum bei der Einschätzung, welche Maßnahmen aufgrund seiner besonderen Umstände jeweils am besten geeignet sind. Der Pakt verlangt eindeutig von jedem Vertragsstaat, alle erforderlichen Maßnahmen zu treffen, um zu gewährleisten, dass jeder Mensch Zugang zu medizinischen Einrichtungen und ärztlicher Betreuung hat, damit ein Höchstmaß an körperlicher und geistiger Gesundheit so bald wie möglich erreicht werden kann. Dies erfordert die Verabschiedung einer nationalen Strategie für die Gewährleistung des Rechts auf Gesundheit für alle, die auf menschenrechtlichen Grundsätzen beruht und die die entsprechenden Ziele definiert, sowie die Erarbeitung politischer Richtlinien und entsprechender Indikatoren und Benchmarks für das Recht auf Gesundheit. Die nationale Gesundheitsstrategie sollte auch die zur Erreichung der Ziele verfügbaren Ressourcen benennen sowie den kostengünstigsten Weg ihrer Nutzung aufzeigen.

54. Die Ausarbeitung und Umsetzung nationaler Gesundheitsstrategien und Aktionspläne sollte unter anderem die Grundsätze des Diskriminierungsverbots und die Mitwirkung der Bevölkerung beachten. Insbesondere muss das Recht von Individuen oder Gruppen, an Entscheidungsprozessen teilzuhaben, die ihre Entwicklung beeinflussen können, ein integraler Bestandteil jeder Politik, jedes Programms und jeder Strategie sein, die entwickelt werden, um den Verpflichtungen gemäß Artikel 12 nachzukommen. Die Pflicht zur Förderung der Gesundheit bedeutet die effektive Mitwirkung der Bevölkerung bei der Prioritätensetzung Entscheidungsfindung, Planung, Umsetzung und Bewertung von Strategien zur Erreichung einer besseren Gesundheit. Eine effektive Versorgung mit Gesundheitsdiensten kann nur dann sichergestellt werden, wenn die Partizipation der Bevölkerung von den Vertragsstaaten garantiert wird.

55. Die nationale Gesundheitsstrategie und der Aktionsplan sollten darüber hinaus auf den Grundsätzen der Rechenschaftspflicht, der Transparenz und der Unabhängigkeit der Gerichtsbarkeit beruhen, da gute Regierungs- und 
Verwaltungsführung (good governance) für die Verwirklichung aller Menschenrechte einschließlich des Rechts auf Gesundheit unerlässlich ist. Um ein positives Umfeld für die Verwirklichung des Rechts auf Gesundheit zu schaffen, sollten die Vertragsstaaten geeignete Maßnahmen treffen, um sicherzustellen, dass sich die Wirtschaft und die Zivilgesellschaft des Rechts auf Gesundheit bewusst sind und dessen Bedeutung bei der Verfolgung ihrer Aktivitäten berücksichtigen.

56. Die Staaten sollten erwägen, Rahmengesetze zu verabschieden, um ihre nationale Gesundheitsstrategie in die Wege zu leiten. Die Rahmengesetze sollten nationale Monitoringmechanismen für die Umsetzung nationaler Gesundheitsstrategien und Aktionspläne festschreiben. Sie sollten Vorschriften für die zu erreichenden Ziele und den für die Erreichung der Ziele vorgesehenen Zeitrahmen enthalten; Vorschriften über die Mittel, mit denen die Benchmarks für das Recht auf Gesundheit eingehalten werden können; für die beabsichtigte Zusammenarbeit mit der Zivilgesellschaft, zum Beispiel mit Gesundheitsexperten/innen, dem Privatsektor und internationalen Organisationen; für die institutionelle Verantwortlichkeit bei der Umsetzung der Gesundheitsstrategie und des Aktionsplans und mögliche Beschwerdeverfahren. Beim Monitoring der Fortschritte in der Verwirklichung des Rechts auf Gesundheit sollten die Staaten solche Faktoren und Schwierigkeiten bezeichnen, die die Umsetzung ihrer Verpflichtungen beeinträchtigen.

\section{Indikatoren und Benchmarks für das Recht auf Gesundheit}

57. Nationale Gesundheitsstrategien sollten geeignete Indikatoren und Benchmarks für das Recht auf Gesundheit festschreiben. Die Indikatoren sollten dazu dienen, die Erfüllung der staatlichen Verpflichtungen gemäß Artikel 12 auf nationaler und internationaler Ebene zu beobachten. Die Staaten können dazu Anleitung hinsichtlich geeigneter Indikatoren für das Recht auf Gesundheit durch die fortlaufende Arbeit der WHO und von UNICEF erhalten. Solche geeigneten Indikatoren für das Recht auf Gesundheit setzten eine Aufschlüsselung nach Diskriminierungsverbotsgründen voraus.

58. Sobald geeignete Indikatoren für das Recht auf Gesundheit bestimmt worden sind, sollten die Vertragsstaaten die jeweiligen nationalen Benchmarks in Bezug auf jeden Indikator festlegen. Im Verlauf der periodischen 
Berichterstattung wird der Ausschuss in einen Prozess des Scoping mit den Vertragsstaaten eintreten. Das Scoping dient der gemeinsamen Erörterung der Indikatoren und nationalen Benchmarks durch den jeweiligen Vertragsstaat und den Ausschuss, die dabei jene Ziele festlegen, die während der nächsten Berichtsperiode erreicht werden sollen. In den folgenden fünf Jahren wird der betreffende Vertragsstaat diese nationalen Benchmarks für das Monitoring der Umsetzung des Artikels 12 benutzen. In dem sich anschließenden Prozess der Berichterstattung werden der Staat und der Ausschuss überprüfen, ob die Benchmarks erreicht wurden oder nicht, und die Gründe für alle Schwierigkeiten erörtern, die dabei möglicherweise aufgetreten sind.

\section{Rechtsbehelfe und Rechenschaftspflicht}

59. Jede Person oder Gruppe, die Opfer einer Verletzung des Rechts auf Gesundheit ist, sollte Zugang zu wirksamen gerichtlichen oder sonstigen geeigneten Rechtsbehelfen auf sowohl nationaler als auch internationaler Ebene haben. ${ }^{28}$ Alle Opfer solcher Verletzungen sollten Anspruch auf angemessene Wiedergutmachung haben, die in Form von Wiederherstellung, Entschädigung, Genugtuung oder von Garantien der Nichtwiederholung erfolgen kann. Innerstaatliche Ombudspersonen, Menschenrechtskommissionen, Verbraucherforen, Vereinigungen für die Rechte von Patienten oder ähnliche Einrichtungen sollten Verletzungen des Rechts auf Gesundheit aufgreifen.

60. Die Übernahme internationaler Rechtsinstrumente, die das Recht auf Gesundheit anerkennen, in die innerstaatliche Rechtsordnung kann den Umfang und die Wirksamkeit von Rechtsbehelfen erheblich verbessern, und die Vertragsstaaten sollten dazu in jedem Fall ermutigt werden. ${ }^{29}$ Dies ermöglicht Gerichten, durch direkte Bezugnahme auf den Pakt über Verlet-

28 Ungeachtet der Frage, ob Gruppen als solche imstande sind, als eigene Rechtssubjekte Rechtsbehelfe in Anspruch zu nehmen, sind die Vertragsstaaten sowohl durch die kollektiven als auch durch die individuellen Dimensionen des Artikel 12 gebunden. Kollektive Rechte sind entscheidend im Bereich von Gesundheit; moderne öffentliche Gesundheitspolitik beruht in einem hohen Maße auf Vorbeugung und Förderung; solche Ansätze sind primär auf Gruppen ausgerichtet.

29 Vgl. Allgemeine Bemerkung Nr. 2 Abs. 9. 
zungen des Rechts auf Gesundheit oder zumindest seiner Kernverpflichtungen zu entscheiden.

61. Richter/innen und Angehörige anderer juristischer Berufe sollten von den Vertragsstaaten ermutigt werden, bei der Wahrnehmung ihrer Aufgaben Verletzungen des Rechts auf Gesundheit größere Aufmerksamkeit zu schenken.

62. Die Vertragsstaaten sollten die Arbeit von Menschenrechtsverteidigern/innen und anderen Mitgliedern der Zivilgesellschaft, die schutzbedürftigen oder ausgegrenzten Gruppen bei der Verwirklichung ihres Rechts auf Gesundheit beistehen wollen, achten, schützen, erleichtern und fördern.

\section{VERPFLICHTUNGEN VON AKTEUREN, DIE KEINE VERTRAGSSTAATEN SIND}

63. Die Rolle der Sonderorganisationen und Programme der Vereinten Nationen und insbesondere die Schlüsselfunktion, die der WHO bei der Verwirklichung des Rechts auf Gesundheit auf internationaler, regionaler und nationaler Ebene zuerkannt wird, ist von ebenso herausragender Bedeutung wie die Aufgaben von UNICEF in Bezug auf das Recht des Kindes auf Gesundheit. Die Staaten sollten technische Hilfe und Zusammenarbeit mit der WHO anstreben, wenn sie ihre jeweilige nationale Strategie zum Recht auf Gesundheit ausarbeiten und umsetzen. Bei der Erarbeitung ihrer Staatenberichte sollten die Vertragsstaaten die umfassenden Informations- und Beratungsdienste der WHO in Bezug auf die Datenerhebung sowie die Aufschlüsselung und Entwicklung der Indikatoren und Benchmarks für das Recht auf Gesundheit in Anspruch nehmen.

64. Ferner sollten koordinierte Bemühungen für die Verwirklichung des Rechts auf Gesundheit unternommen werden, um die Interaktion der jeweiligen Akteure, einschließlich der unterschiedlichen Teile der Zivilgesellschaft, zu verbessern. Entsprechend Artikel 22 und 23 des Pakts sollten die WHO, die Internationale Arbeitsorganisation (ILO), das Entwicklungsprogramm der Vereinten Nationen (UNDP), UNICEF, der Bevölkerungsfonds der Vereinten Nationen (UNFPA), die Weltbank, die regionalen Entwick- 
lungsbanken, der internationale Währungsfonds (IMF), die Welthandelsorganisation (WTO) und andere relevante Organisationen im System der Vereinten Nationen ihre Zusammenarbeit mit den Vertragsstaaten zur Verwirklichung des Rechts auf Gesundheit auf nationaler Ebene unter gebührender Berücksichtigung ihrer jeweiligen Mandate und auf der Grundlage ihrer jeweiligen Fachkenntnisse verstärken. Insbesondere sollten die internationalen Finanzinstitutionen, vor allem die Weltbank und der Internationale Währungsfond, dem Schutz des Rechts auf Gesundheit in ihrer Finanzpolitik, den Kreditvereinbarungen und Strukturanpassungsprogrammen größere Beachtung schenken. Bei der Prüfung der Staatenberichte wird sich der Ausschuss damit befassen, wie sich die von allen Akteuren, die keine Vertragsstaaten sind, erwiesene Hilfe auf die Fähigkeit der Staaten zur Erfüllung ihrer Verpflichtungen gemäß Artikel 12 auswirkt. Die Verfolgung eines menschenrechtsbezogenen Ansatzes durch die Sonderorganisationen, Programme und Organe der Vereinten Nationen wird die Verwirklichung des Rechts auf Gesundheit in hohem Maße erleichtern. Im Verlauf seiner Überprüfung der Berichte der Vertragsstaaten wird der Ausschuss auch die Rolle der Berufsverbände im Gesundheitssektor und sonstiger nichtstaatlicher Organisationen einbeziehen, die mit den Verpflichtungen der Vertragsstaaten gemäß Artikel 12 in Zusammenhang stehen.

65. Die Rolle der WHO, des Amts des Hohen Flüchtlingskommissars der Vereinten Nationen, des Internationalen Komitees vom Roten Kreuz sowie der nichtstaatlichen Organisationen und nationalen ärztlichen Vereinigungen ist in Bezug auf Katastrophenhilfe und humanitäre Hilfe in Zeiten des Notstandes einschließlich der Hilfe für Flüchtlinge und Binnenvertriebene von besonderer Bedeutung. Bei der Versorgung mit internationaler medizinischer Hilfe und bei der Verteilung und Verwaltung von Hilfsgütern, zum Beispiel von sicherem Trinkwasser, Nahrungsmitteln und Arzneimitteln, sowie bei der finanziellen Hilfe sollte den am meisten schutzbedürftigen und ausgegrenzten Gruppen der Bevölkerung Vorrang eingeräumt werden.

CESCR E/C.12/2002/11

20. Januar 2003 



\section{Autorinnen und Autoren}

Prof. Dr. phil. Dr. h.c. Heiner Bielefeldt

Lehrstuhl für Menschenrechte und Menschenrechtspolitik

Friedrich-Alexander-Universität Erlangen-Nürnberg

Kochstraße 4

91054 Erlangen

\section{Lotta Eriksson}

Swedish National Council on Medical Ethics

Ministry of Health and Social Affairs

10333 Stockholm

Schweden

Prof. Dr. med. Andreas Frewer, M.A.

Professur für Ethik in der Medizin

Friedrich-Alexander-Universität Erlangen-Nürnberg

Glückstraße 10

91054 Erlangen

cand. med. Christina Heinicke

Doktorandin an der Professur für Ethik in der Medizin

Friedrich-Alexander-Universität Erlangen-Nürnberg

Glückstraße 10

91054 Erlangen 


\section{PD Dr. phil. Michael Krennerich}

Lehrstuhl für Menschenrechte und Menschenrechtspolitik

Friedrich-Alexander-Universität Erlangen-Nürnberg

Kochstraße 4

91054 Erlangen

Dr. phil. Amrei Müller

Department of Public and International Law

Postboks 6706

St. Olavs plass

0130 Oslo

Norwegen

Dr. med. Andreas Reis, M.Sc.

World Health Organization

20, Avenue Appia

1211 Geneva 27

Schweiz

Dr. Abha Saxena, M.D.

World Health Organization

20, Avenue Appia

1211 Geneva 27

Schweiz

Dr. phil. fac. theol. Martina Schmidhuber

Professur für Ethik in der Medizin

Friedrich-Alexander-Universität Erlangen-Nürnberg

Glückstraße 10

91054 Erlangen

\section{PD Dr. phil. Caroline Welsh}

Rheinstraße 10

12159 Berlin 